\section{Pacific Northwest}

National Laboratory

Operated by Battelle for the

U.S. Department of Energy

\title{
DUSTRAN 1.0 User's Guide: \\ A GIS-Based Atmospheric Dust Dispersion Modeling System
}

\author{
K. J. Allwine \\ F. C. Rutz \\ W. J. Shaw \\ J. P. Rishel \\ B. G. Fritz \\ E. G. Chapman \\ B. L. Hoopes \\ T. E. Seiple
}

September 2006

Prepared for the U.S. Department of Defense Strategic Environmental Research and Development Program under a Related Services Agreement with the U.S. Department of Energy under contract DE-AC05-76RL01830. 


\title{
DUSTRAN 1.0 User's Guide: A GIS-Based Atmospheric Dust Dispersion Modeling System
}

\author{
K. J. Allwine \\ F. C. Rutz \\ W. J. Shaw \\ J. P. Rishel \\ B. G. Fritz \\ E. G. Chapman \\ B. L. Hoopes \\ T. E. Seiple
}

September 2006

Prepared for the U.S. Department of Defense Strategic Environmental Research and Development Program under a Related Services Agreement with the U.S. Department of Energy under contract DE-AC05-76RL01830.

Pacific Northwest National Laboratory

Richland, Washington 99352 


\section{Summary}

The U.S. Department of Energy's Pacific Northwest National Laboratory just completed a multi-year project to develop a fully tested and documented atmospheric dispersion modeling system (DUST TRANsport or DUSTRAN) to assist the U.S. Department of Defense (DoD) in addressing particulate air quality issues at military training and testing ranges. The project was primarily funded by DoD's Strategic Environmental Research and Development Program with additional funding from the U.S. Forest Service and U.S. Environmental Protection Agency (EPA) to address their issues related to the "off-target" drift of aerially applied pesticides.

DUSTRAN is constructed from widely-used, scientifically-defensible atmospheric models and model components. The modeling system efficiently couples these modeling components and advances the state-of-science in dust-emission formulations. DUSTRAN is based on Environmental System Research Institute's ArcMap Geographic Information System (Version 9.x), the EPA-approved CALifornia PUFF (CALPUFF) dispersion model, and the widely used CALifornia GRID (CALGRID) dispersion model. The CALifornia METeorological (CALMET) model provides the meteorological fields (e.g., winds, mixing height) for the CALPUFF and CALGRID dispersion models. DUSTRAN includes dust-emission modules for creating source-term factors from both wind-blown dust generation and wheeled military vehicle activities. The primary features of DUSTRAN are:

- The modeling domain is graphically specified and is size selectable (20 km to $400 \mathrm{~km})$.

- It operates at any U.S. geographic location and has an "Add Site" wizard that generates a new site's supporting files and data structure for use in a simulation.

- Single-station or multiple-station meteorology can be used and easily specified.

- Multiple point, area, and line releases can be accommodated and specified graphically.

- Simulation and release times are easily specified in the user interface.

- The output concentrations and deposition contours can be viewed graphically, and the output can be animated to view the progression of the plume across the modeling domain.

- Multiple particle sizes and gaseous species can be simulated at one time.

- Simulation periods are typically a few hours to a few days.

- The atmospheric models treat wet and dry deposition and complex terrain effects.

This manual documents the DUSTRAN modeling system and includes installation instructions, a user's guide, and detailed example tutorials. 



\section{Acronyms}

APGEMS

CALGRID

CALMET

CALPOST

CALPUFF

CST

DEM

DoD

DOE

DRI

DUSTRAN

EPA

ESRI

EST

FS

FSL

GIS

GLCC

M-O

MST

NARCS

NOAA

NWS

NTC

OWE

PGEMS

PGT

PM

PNNL

PST
Air Pollutant Graphical Environmental Modeling System

CALifornia photochemical GRID model

CALifornia METeorological model

CALifornia POST-processing program

CALifornia PUFF model

Central Standard Time

digital elevation model

U.S. Department of Defense

U.S. Department of Energy

Desert Research Institute

DUST TRANsport

U.S. Environmental Protection Agency

Environmental System Research Institute

Eastern Standard Time

U.S. Forest Service

Forecast Systems Laboratory

geographic information system

global land cover characteristics

Monin-Obukhov (similarity theory)

Mountain Standard Time

number of arc distances

National Oceanic \& Atmospheric Administration

National Weather Service

National Training Center

Olson World Ecosystem (database)

Pacific Gas and Electric Modeling System

Pasquill-Gifford-Turner specifications

particulate matter

Pacific Northwest National Laboratory

Pacific Standard Time 
SERDP

SPRAYTRAN

USGS

UTM
Strategic Environmental Research and Development Program

Spray TRANsport modeling system

U.S. Geological Survey

Universal Transverse Mercator 


\section{Acknowledgments}

This research was supported in part by the U.S. Department of Defense through the Strategic Environmental Research and Development Program (SERDP) under a Related Services Agreement with the U.S. Department of Energy (DOE) under Contract DE-AC05-76RL01830. Pacific Northwest National Laboratory (PNNL) is operated for DOE by Battelle.

Ms. Crystal Driver and Mr. Randy Kirkham of PNNL provided field data from studies conducted at the National Training Center, Fort Irwin, California, for initial testing of the modeling system. These studies were conducted by PNNL through funding from the U.S. Army Forces Command. Dr. Jack Gillies of Desert Research Institute (DRI) provided emission-factor data for wheeled military vehicles. The emission factors were developed under an existing Strategic Environmental Research and Development Program project (CP-1191) with DRI.

Dr. Bob Holst, the SERDP program manager, provided invaluable guidance and support during all phases of the development of DUSTRAN. Additionally, Dr. Harold Thistle of the U.S. Forest Service and Ms. Sandy Bird of the U.S. Environmental Protection Agency provided important guidance and insight in adapting DUSTRAN to treat the off-target drift of pesticides. Dr. Jim Droppo of PNNL

provided valuable reviews and guidance during completion and final testing of the modeling system. 



\section{Contents}

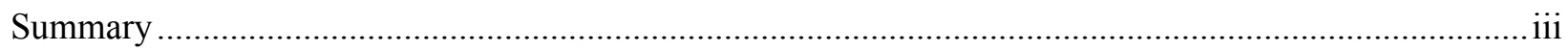

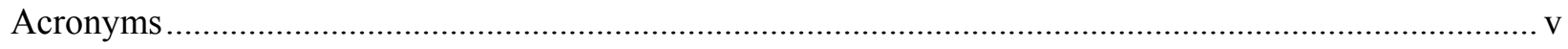

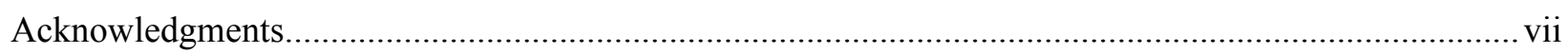

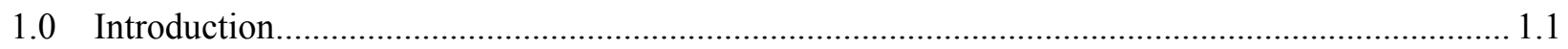

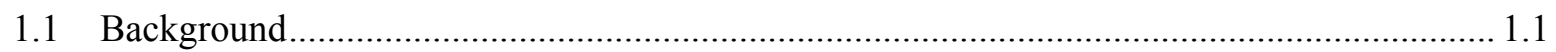

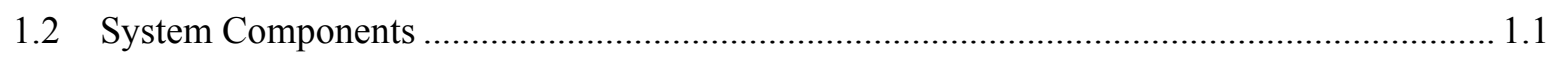

2.0 Technical Overview of the DUSTRAN Modeling System..................................................... 2.1

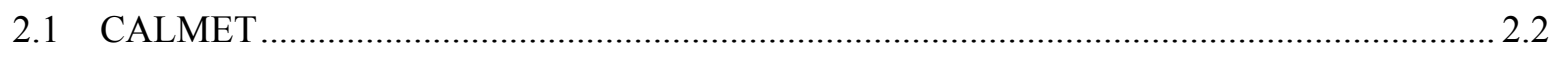

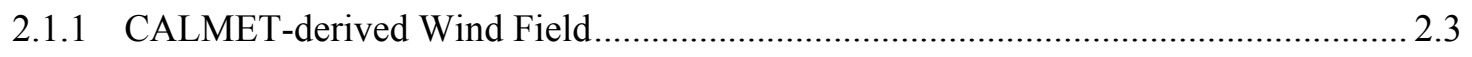

2.1.2 CALMET-Derived Boundary Layer Parameters .................................................... 2.5

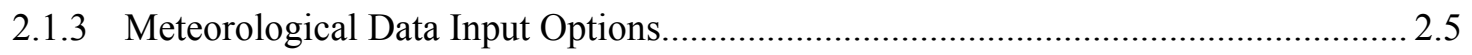

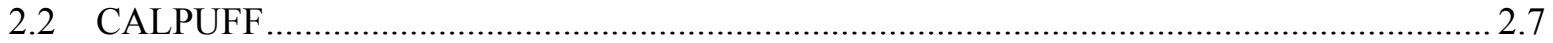

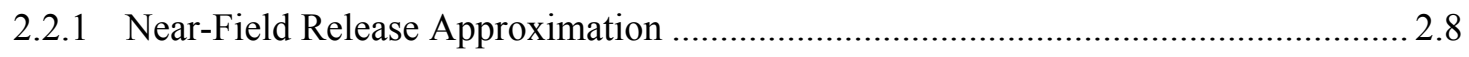

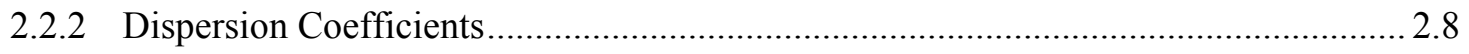

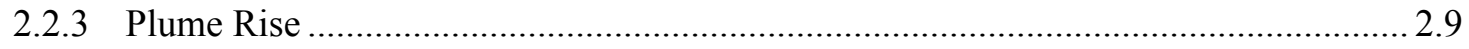

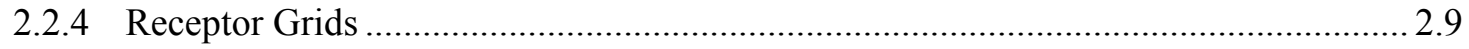

2.2.5 Representing Moving Vehicles as Line and Area Dust Sources............................. 2.10

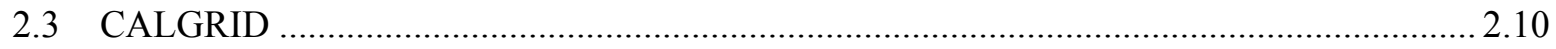

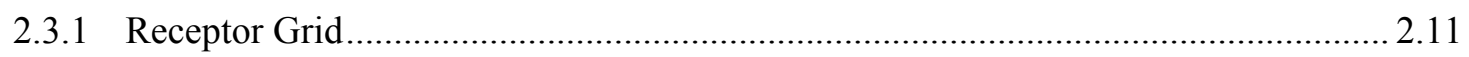

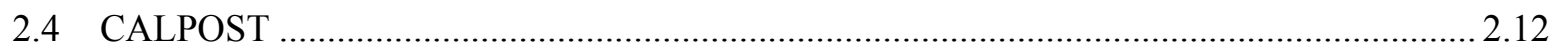

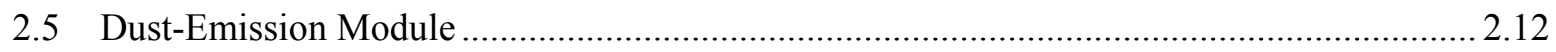

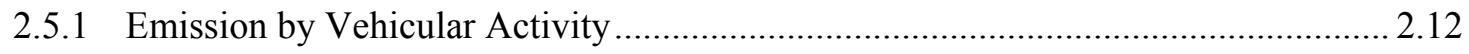

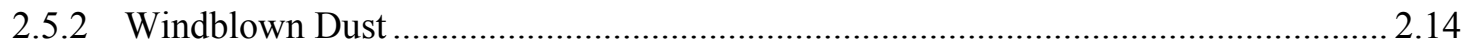

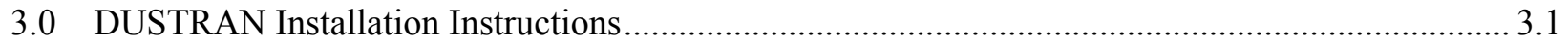

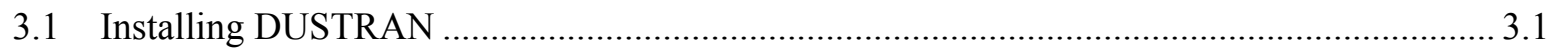

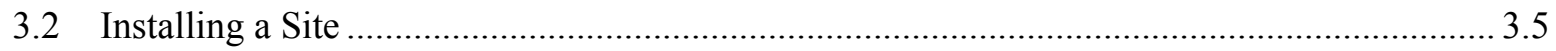

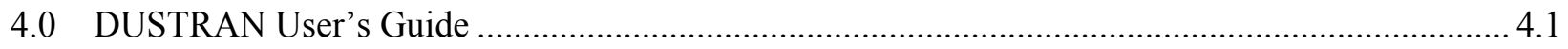

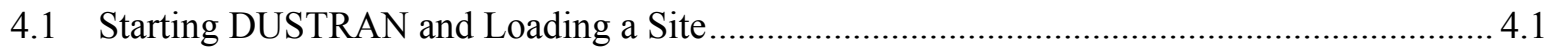

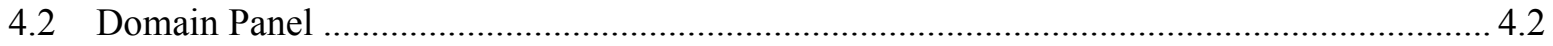




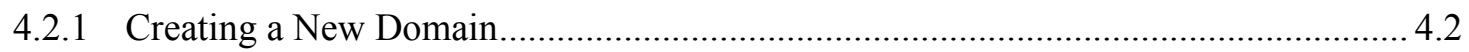

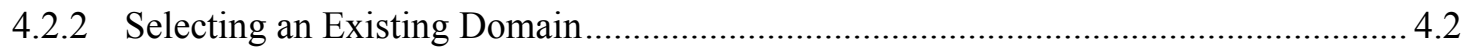

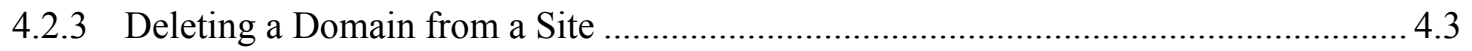

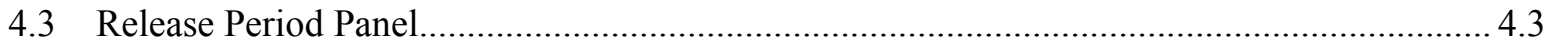

4.3.1 Setting the Default Release Start Time and Duration ........................................... 4.3

4.3.2 Synchronizing the Release Start Time and Duration for all Sources.......................... 4.4

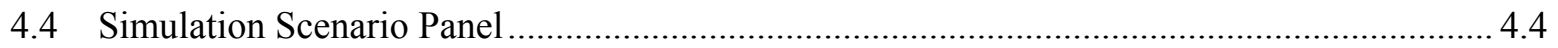

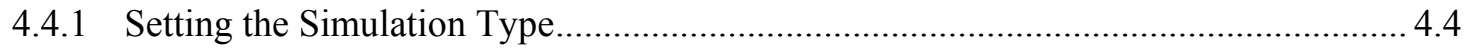

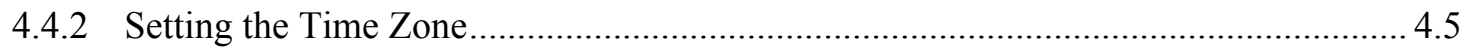

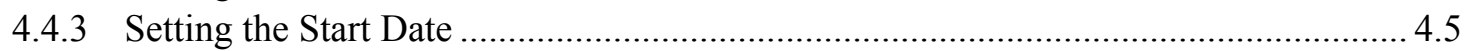

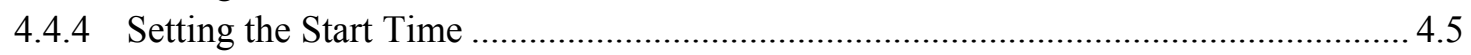

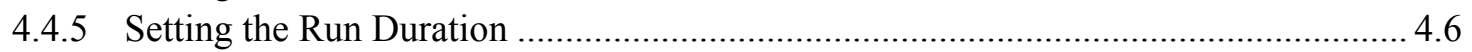

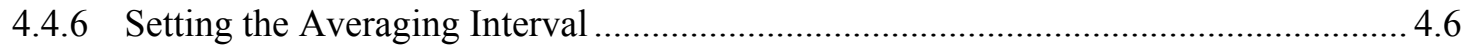

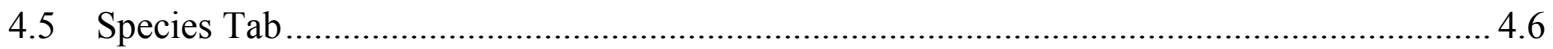

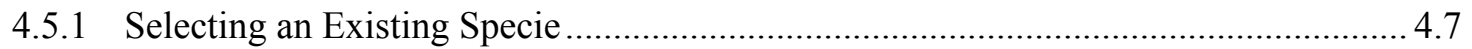

4.5.2 Modify Data for an Existing Specie....................................................................... 4.7

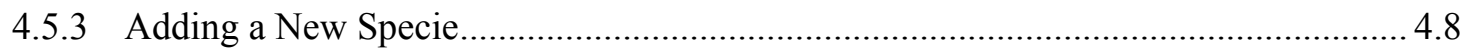

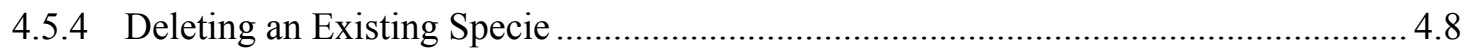

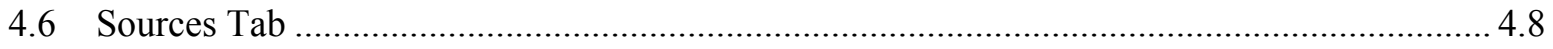

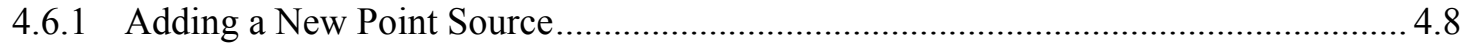

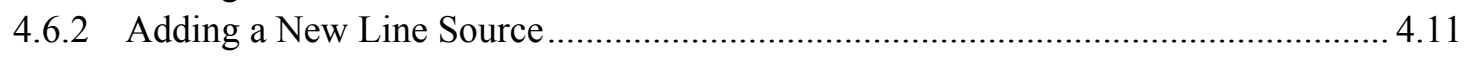

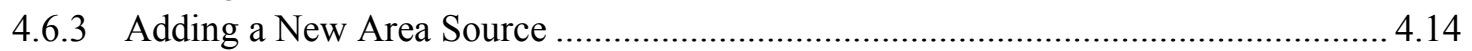

4.6.4 Selecting Existing Sources to Use in a Simulation .............................................. 4.18

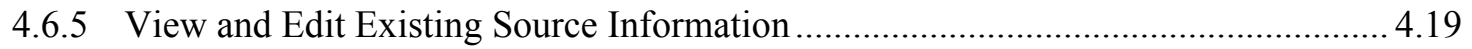

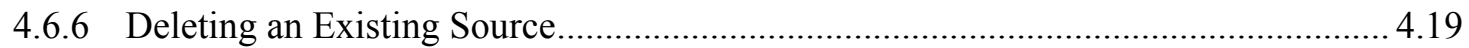

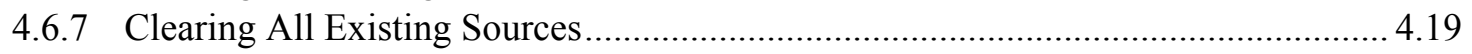

4.6.8 Saving Existing Sources as a Scenario .................................................................. 4.19

4.6.9 Adding Characteristic Files (Soil and Vegetation for Wind-blown Dust) ................. 4.19

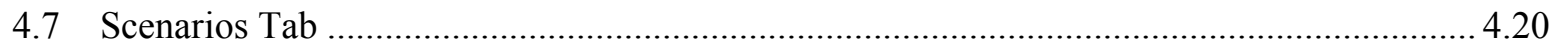

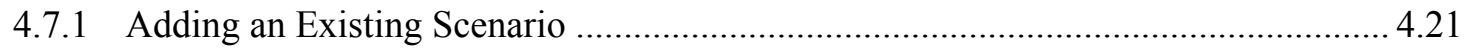

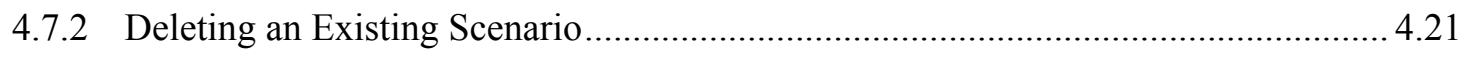

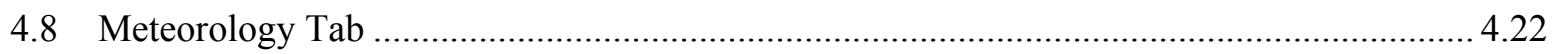

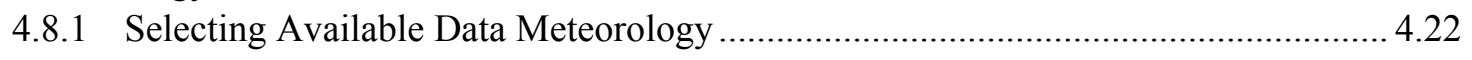

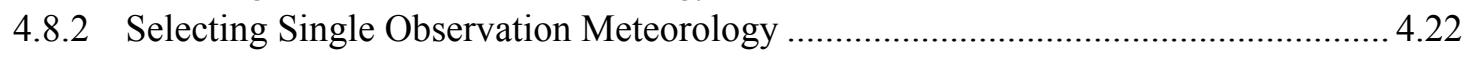

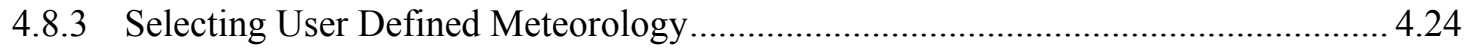

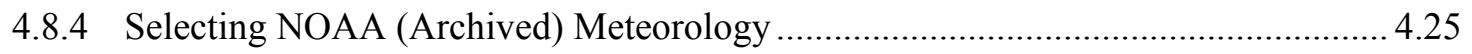

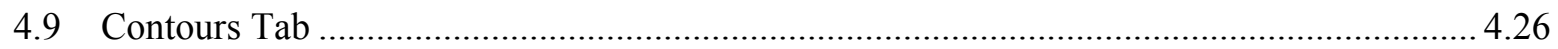

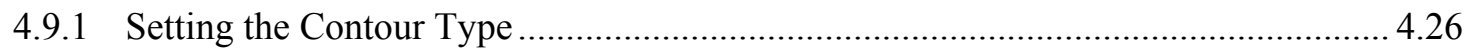

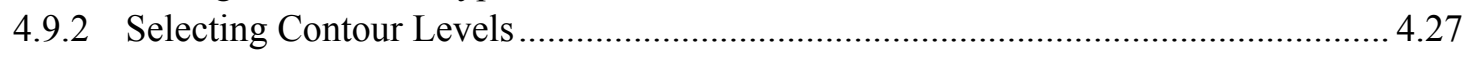

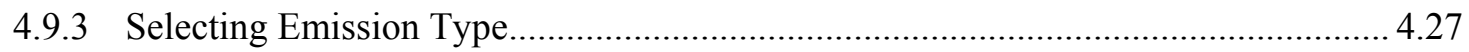

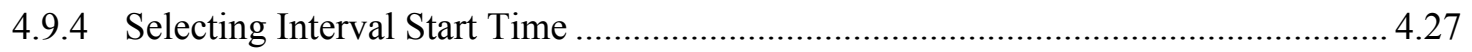




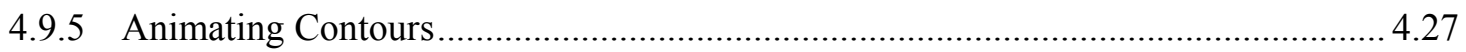

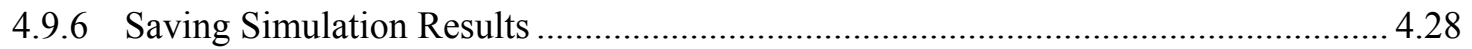

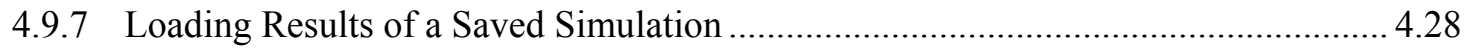

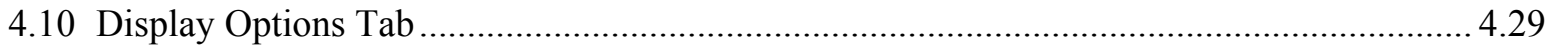

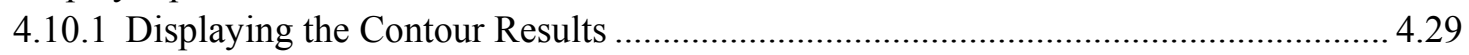

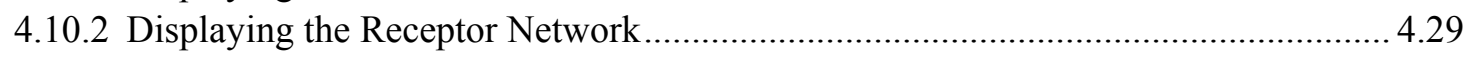

4.10.3 Displaying the Calculated Wind Vector Field ........................................................... 4.30

4.10.4 Displaying Surface and Upper-Air Meteorological Station Locations ....................... 4.30

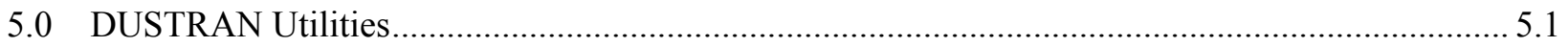

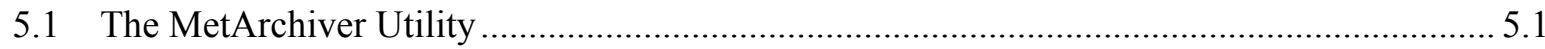

5.1.1 Starting the MetArchiver Application........................................................................... 5.1

5.1.2 Configuring MetArchiver with Windows Task Scheduler ............................................ 5.5

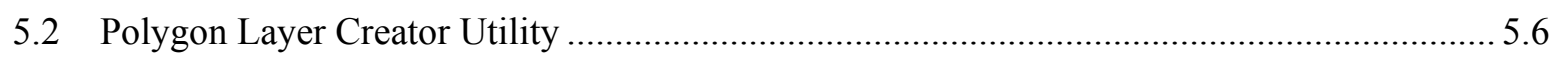

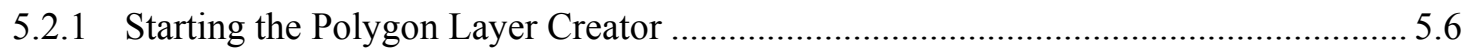

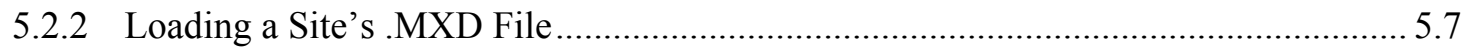

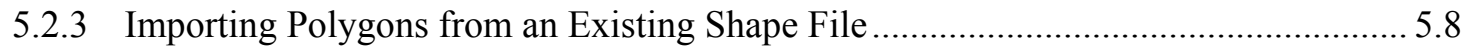

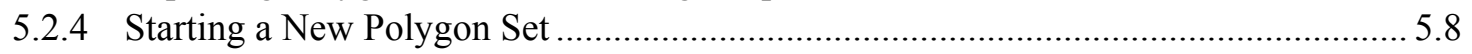

5.2.5 Site Navigation within Map Display Window ........................................................ 5.9

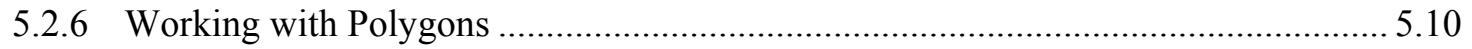

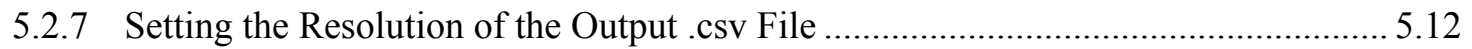

5.2.8 Creating the Output Shape File and .csv File........................................................ 5.13

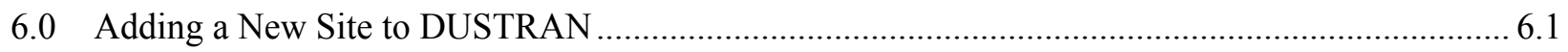

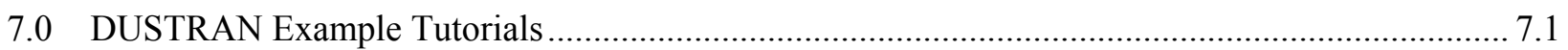

7.1 Simulating Dust Dispersion from Source Emissions....................................................... 7.1

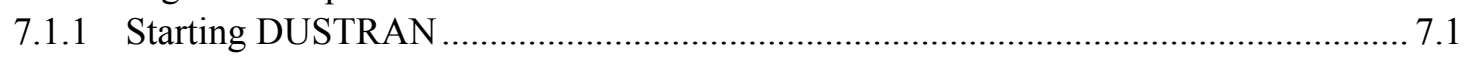

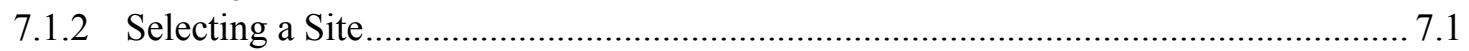

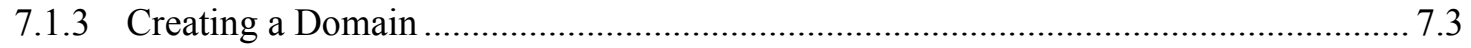

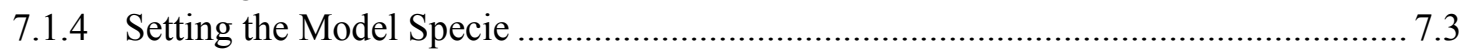

7.1.5 Creating a Point Source .......................................................................................... 7.4

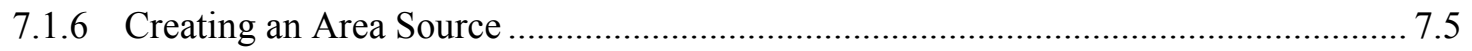

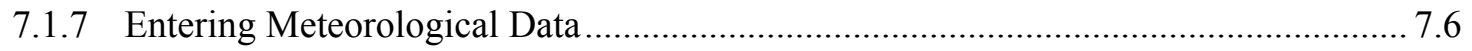

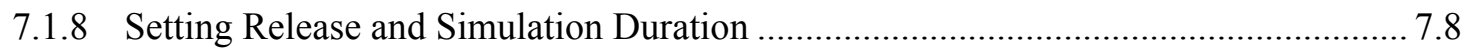

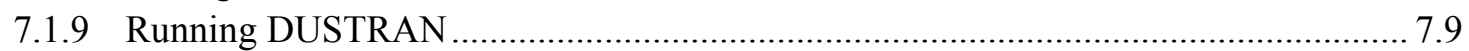

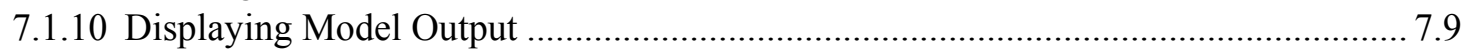

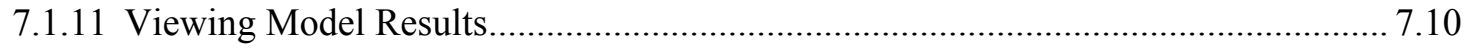

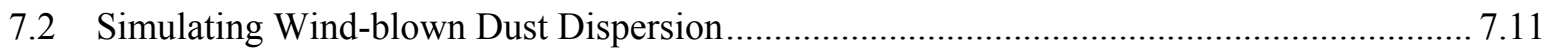

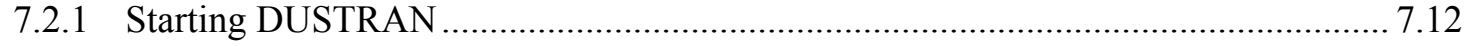

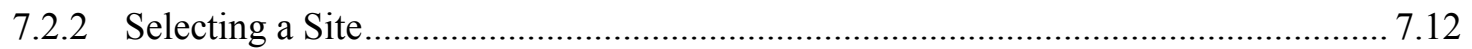

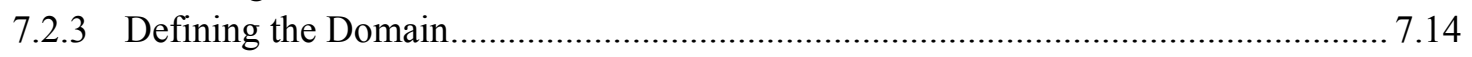

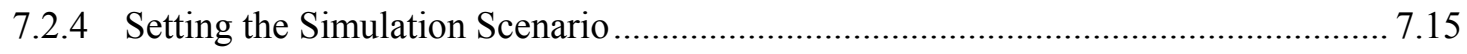


7.2.5 Setting the Soil and Vegetation Characteristic Files........................................... 7.16

7.2.6 Viewing the Soil and Vegetation Characteristic Files ........................................... 7.17

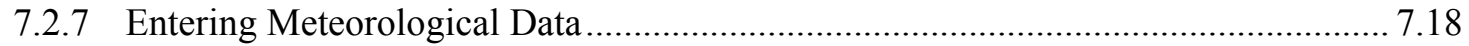

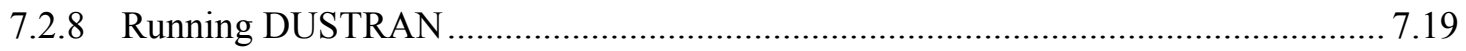

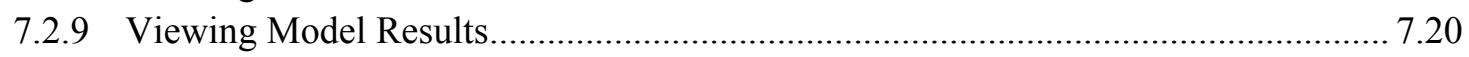

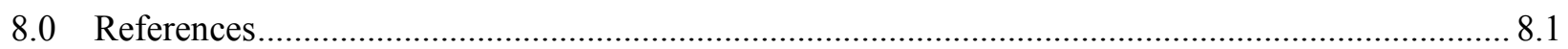

Appendix A: DUSTRAN Directory and File Documentation ......................................................... A.1

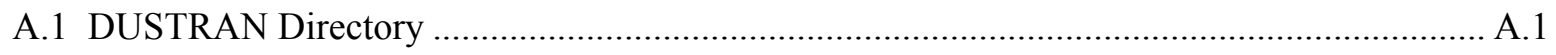

A.2 Site Directory Static Data Files.................................................................................... A.7

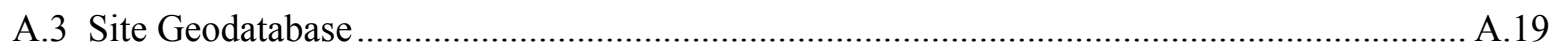

A.4 DUSTRAN Initialization File (DUSTRAN.ini) …....................................................... A.24

A.5 Emission Model Input and Output Files …................................................................. A.26 


\section{Figures}

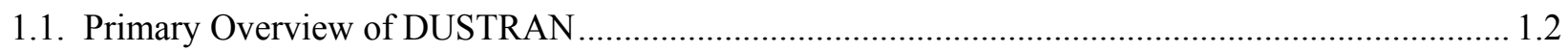

2.1. Dispersion Model Components within the DUSTRAN Modeling System......................................2.1

2.2. Data Flow and Geophysical Preprocessors for CALMET ….................................................... 2.4

2.3. Example of the Primary Cartesian Receptor Grid and a Polar and Cartesian Sub-Grid Used Within CALPUFF.

2.4. Contour Plot of RMS Differences Between Equation (2.7) and Observations of Dust Flux G Discussed by Gillette and Passi (1988) ................................................................................ 2.16

2.5. Observations of G Versus $u^{*}$ after Gillette and Passi $(1988)$.................................................. 2.16

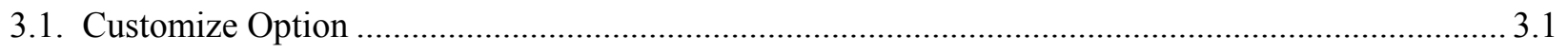

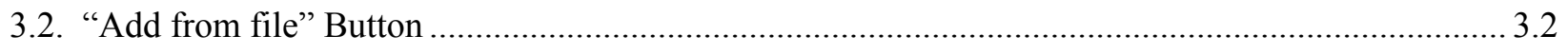

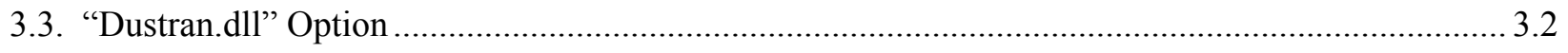

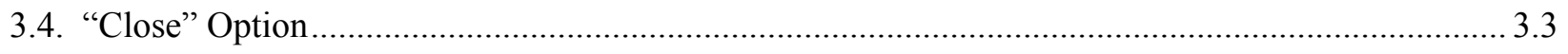

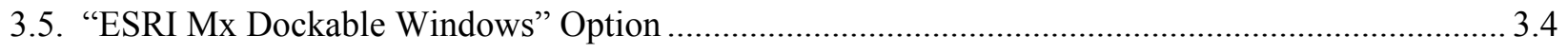

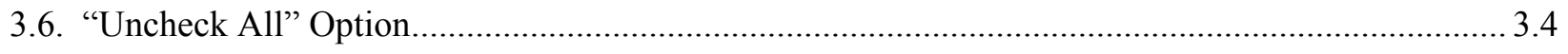

4.1. Site Loaded into the DUSTRAN Modeling System ................................................................ 4.1

4.2. Example Domain Panel with Controls Labeled ...................................................................... 4.2

4.3. Example Release Period Panel with Controls Labeled ............................................................ 4.3

4.4. Example Simulation Scenario Panel with Controls Labeled …................................................... 4.4

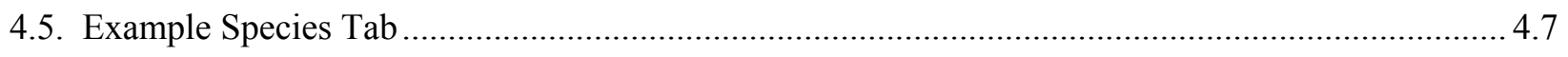

4.6. Example Sources Tab with Point, Line, and Area Sources Entered ............................................. 4.9

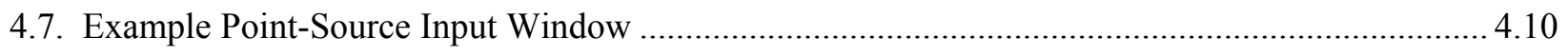

4.8. Example Line-Source Input Window Using the User Defined Emission Option .......................... 4.12

4.9. Example Vehicle Input Window for a Line Source …......................................................... 4.14

4.10. Example Area-Source Input Window Using the User Defined Emission Option ....................... 4.16

4.11. Example Vehicle Input Window for an Area Source........................................................... 4.17 
4.12. Characteristics Priorities Dialog Box for Adding Files, such as Soil and Vegetation Classes, in DUSTRAN

4.13. Example Scenario Tab with Controls Labeled

4.14. Onsite Data Selected Through the "Available Data" Option on the "Meteorology" Tab.

4.15. Single Observation Meteorology Input Window

4.16. Sample "Surface Stations" Tab for Specifying CALMET-Ready Station Locations in DUSTRAN.

4.17. Example Contours Tab with Controls Labeled

4.18. Animation Control with Buttons Labeled

4.19. Example Prior Run Selection Window

4.20. Display Options Tab

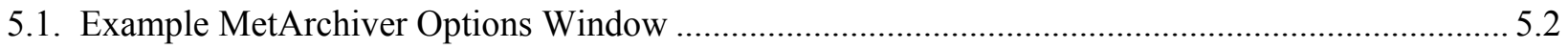

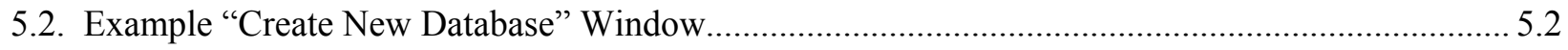

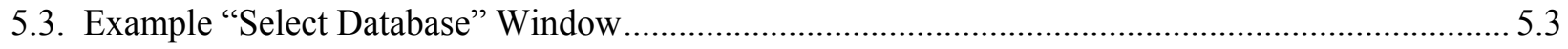

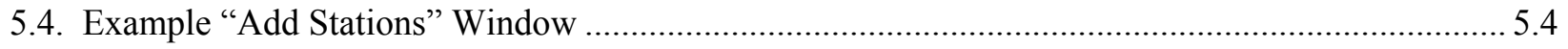

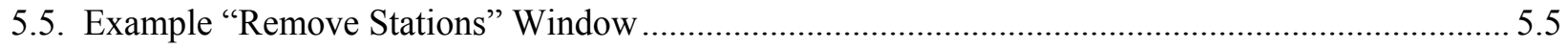

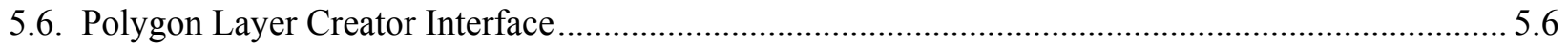

5.7. Launching Polygon Layer Creator from Within DUSTRAN …................................................. 5.7

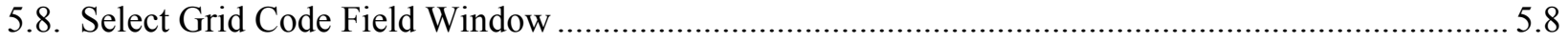

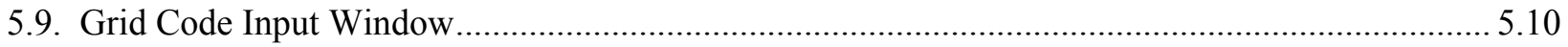

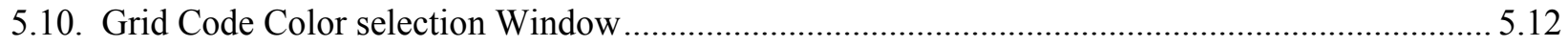

5.11. Polygon Grid Code Value Window …….......................................................................... 5.12

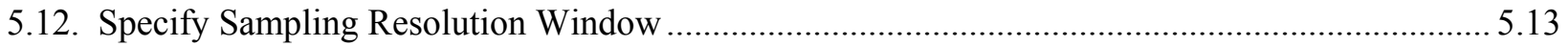

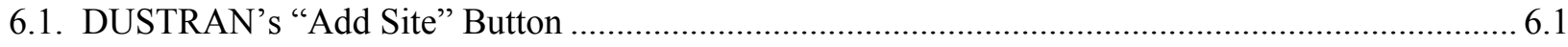

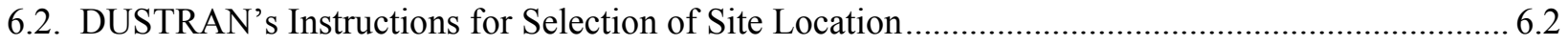

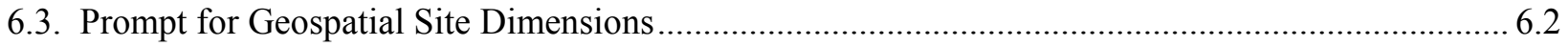

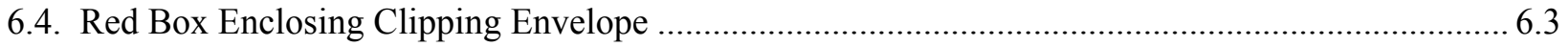




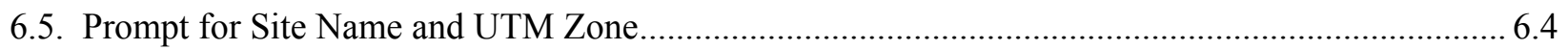

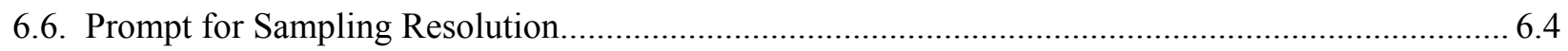

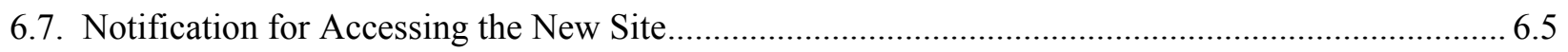

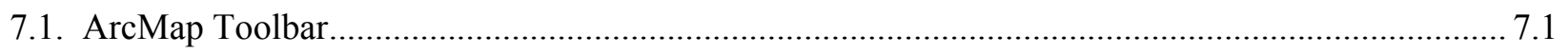

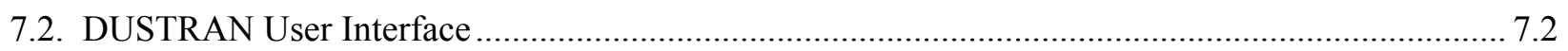

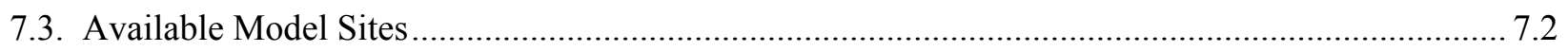

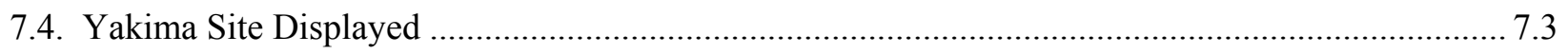

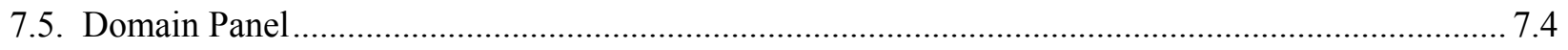

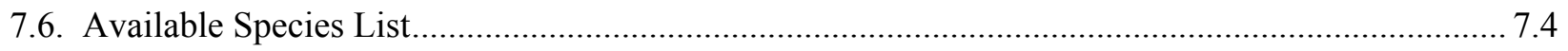

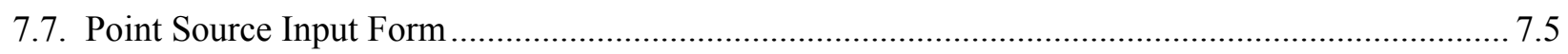

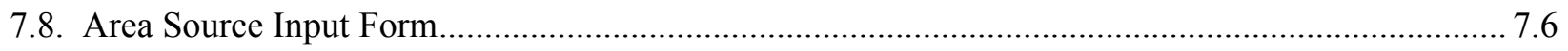

7.9. Information Required Under the Vehicle Parameters Tab....................................................... 7.7

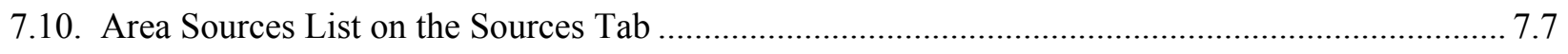

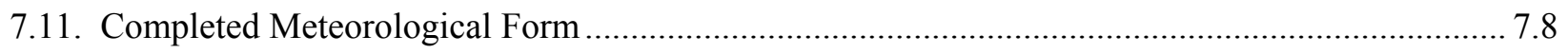

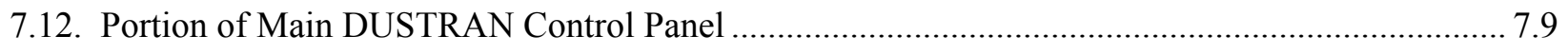

7.13. "Run Simulation” Button for Running DUSTRAN Simulation ............................................... 7.9

7.14. Choices After Clicking the "Display Options" Tab..............................................................10

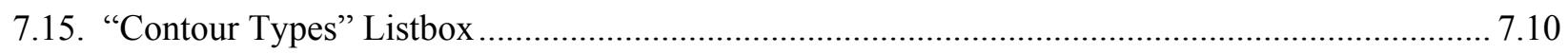

7.16. Display of Concentration Contours and Wind Vectors for the Hour from 9-10 AM .................. 7.11

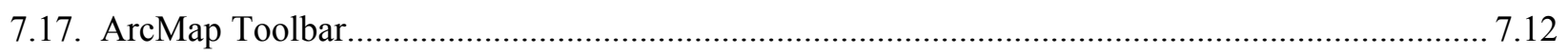

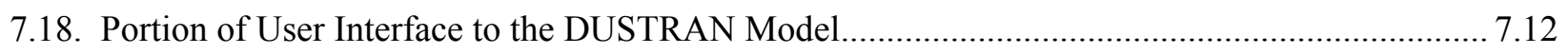

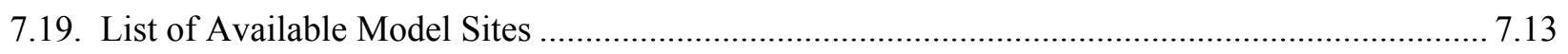

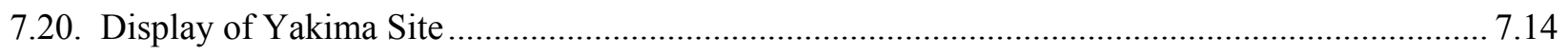

7.21. Display of 200-km-Square Yakima Domain Within Yakima Site............................................. 7.15

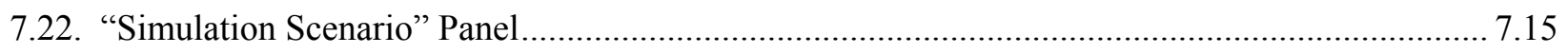

7.23. Adding Soil and Vegetation Files from DUSTRAN User Interface ....................................... 7.16 


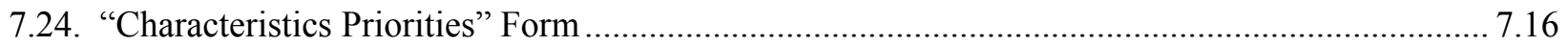

7.25. Display Showing Choices for Soil and Vegetation Layers ................................................... 7.17

7.26. Display of Olson Vegetation Layer for the Yakima Site ........................................................... 7.18

7.27. Completed "Specify Meteorological Data" Form .....................................................................19

7.28. "Run Simulation” Button in DUSTRAN User Interface ............................................................ 7.19

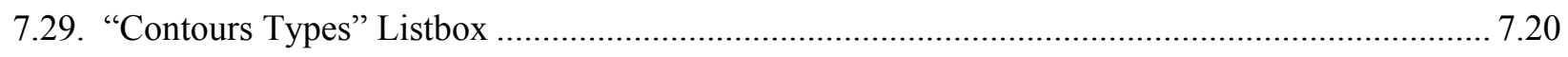

7.30. Display of Concentration Contours for the Hour from 7-8 AM ............................................. 7.21

\section{Tables}

2.1. Version Numbers for Model Components Implemented in DUSTRAN 1.0 ...............................2.2

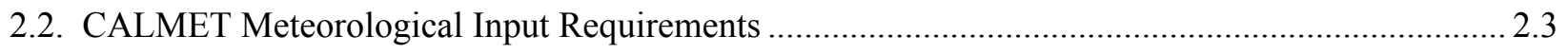

2.3. Olson Vegetation Classes Used in the Wind-blown Dust-Emission Model ................................. 2.18

2.4. Features of Typical Dust Particles (after Nickovic et al. 2001) ................................................. 2.19

2.5. Fractions, $\beta_{j, k}$, of the Soil Texture Classes in each Zobler Soil Category .................................. 2.19 


\subsection{Introduction}

\subsection{Background}

Activities at U.S. Department of Defense (DoD) training and testing ranges can be sources of dust in local and regional air sheds governed by air quality regulations. Activities that generate dust by disturbing local surfaces include vehicle and troop maneuvers, convoy movement, helicopter activities, munitions impacts, roadway preparations, and wind erosion. Other sources of particulates include the use of smokes and obscurants, controlled burns, and engine operations.

Beginning January 2001, a multi-year research project was started to develop an atmospheric dispersion modeling system to assist the DoD in addressing particulate air quality issues at military training and testing ranges. The effort at the U.S. Department of Energy's Pacific Northwest National Laboratory (PNNL) was funded primarily by DoD's Strategic Environmental Research and Development Program (SERDP). The U.S. Forest Service (FS) and U.S. Environmental Protection Agency (EPA) have also provided funds towards developing the atmospheric modeling system. The FS and the EPA need to address issues related to the "off-target" drift of aerially applied pesticides. They consequently have funded a portion of the development program for the modeling-system user interface and funded wholly the development of a separate pesticide-source-term module. Allwine et al. (2006) describe the Spray TRANsport modeling system (SPRAYTRAN) modeling system used to assess the off-target drift of aerially applied pesticides.

The culmination of this work has led to the development of the DUST TRANsport, or DUSTRAN, modeling system. The basic objectives in formulating DUSTRAN were to (1) identify and construct the system from widely available, scientifically defensible models and model components, (2) couple and integrate the models within a user-friendly geographic information system (GIS) interface, (3) develop and implement an advanced dust-emission model into the modeling system, and (4) document the system through technical articles and a supporting user guide. This manual supports the final objective.

\subsection{System Components}

The DUSTRAN user interface integrates the input and output operations needed for evaluating potential air quality impacts. The three primary components of DUSTRAN and their linkages, illustrated in Figure 1.1, have the following functionalities:

- $\underline{G I S}$ for specifying geographical inputs (e.g., modeling domain, surface characteristics, activity locations), and for viewing model outputs (e.g., spatially and temporally varying dust concentration and deposition fields).

- $\quad$ Dust-emission model for determining dust-emission rates as a function of time and location for various dust-generating activities specified in a simulation.

- $\quad$ Dust-dispersion models for simulating dispersion and deposition to determine the ground-level air concentration and deposition patterns. 
The GIS component is based on the Environmental System Research Institutes' (ESRI's) ArcMap GIS (Version 9.x). DUSTRAN can be configured for application at any U.S. geographical location. New sites can be added using an "Add Site" wizard that creates the required files and data structures. The GIS user interface implemented in DUSTRAN includes the capability to graphically define simulations by:

- Creating a model domain and its surface characteristics

- Specifying modeling domain size by selecting dimensions ranging from 20 to $400 \mathrm{~km}$

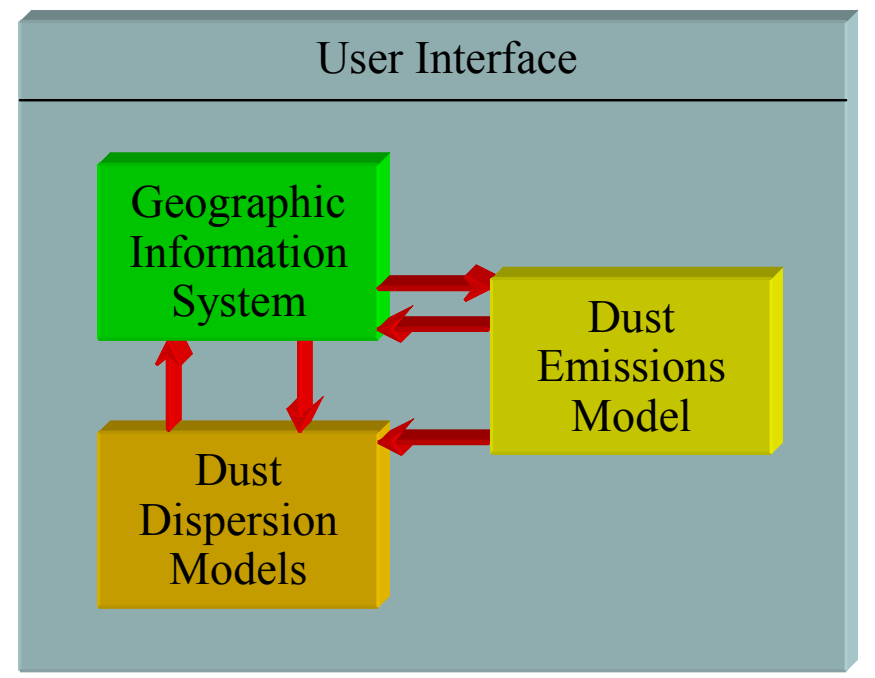

Figure 1.1. Primary Overview of DUSTRAN

- Specifying model simulation and activity release start times and durations

- Specifying activity details in terms of multiple point, area, and line releases.

The GIS output functionality includes the capability to graphically view spatial patterns of ground-level concentration and deposition, including an option to view a temporal animation of these patterns. Additionally, patterns of wind vectors for selected heights above ground can be viewed graphically.

The dust-emission model uses vehicular dust-emission factors developed on SERDP project CP-1191 (Gillies et al. 2005a, 2005b) and incorporates the widely used AP-42 emission factors for paved and unpaved roads (EPA 2005). Additionally, wind-blown dust-source strengths are based on current formulations in the scientific literature. The dust-emission model functionality includes creating release rates for:

- Wind-blown dust generation

- Wheeled military vehicle activities.

The definition of emission rates is integrated into the GIS. The user interface includes the ability to graphically define source areas of wind-blown dust as well as area- and line-source characteristics (such as type, speed, and number of vehicles) associated with vehicular dust emission.

The air dispersion models implemented in DUSTRAN are the EPA-approved CALifornia PUFF (CALPUFF) model (Scire et al.2000a) and the widely-used CALifornia photochemical GRID

(CALGRID) model (Scire et al.2000b). The functionality of the DUSTRAN implementation of these air dispersion models includes: 
- Easy selection of single or multiple station meteorology

- Use of temporally and spatially varying wind fields

- Air flow models accounting for terrain influences

- Accounting for wet and dry deposition processes

- Typical simulation periods of a few hours to a few days

- Consideration of multiple particles size ranges in a single simulation

- Ability to specify combinations of receptor grids, including polar grids for point sources and specialized rectangular grids for area sources.

The CALGRID model is used for wind-blown dust simulations because the entire modeling domain is a potential source of dust, necessitating the use of a grid model for computational efficiency. For simulating dust dispersion and deposition from various specific point, line, and areas sources, the CALPUFF model is used, giving better definition of near-source (within $1 \mathrm{~km}$ ) impacts. 


\subsection{Technical Overview of the DUSTRAN Modeling System}

DUSTRAN is a comprehensive dispersion modeling system, consisting of a dust-emissions module, a diagnostic meteorological model, and dispersion models that are integrated seamlessly into ESRI's ArcMap GIS. DUSTRAN functions as a console application within ArcMap and allows the user to interactively create a release scenario and run the underlying models. Through the process of data layering, the model domain, sources, and results - including the calculated wind vector field and plume contours - can be displayed with other spatial and geophysical data sources to aid in analyzing and interpreting the scenario.

Fundamental to DUSTRAN is a dust-emissions model that includes algorithms for calculating dust emissions from both active and natural sources. Active sources include vehicular dust generation from paved/unpaved roadway surfaces as well as emission factors for various wheeled military vehicles (Gillies et al. 2005a, 2005b) and the widely-used AP-42 emission factors (EPA 2005). Natural sources include wind-blown dust generation from a user-specified domain, and are a function of surface wind stress, soil type, and vegetation type. In either case, dust emissions are calculated for explicit particle size classes; $\mathrm{PM}_{2.5}, \mathrm{PM}_{10}, \mathrm{PM}_{15}$, and $\mathrm{PM}_{30}$. DUSTRAN also allows the user to simulate dispersion and deposition for any desired particle sizes and release rates specified through the user interface. Dispersion and deposition can also be simulated for user-specified gases.

In DUSTRAN, dust transport, diffusion, and deposition are simulated using one of two regulatory (40 CFR Part 51, Appendix W) dispersion models - CALPUFF or CALGRID. The use of two dispersion models arises from their frame-of-reference used in calculating plume transport, which leads to inherent strengths in simulating different source types. The CALPUFF (Scire et al., 2000a) dispersion model is used for vehicle-generated dust emissions where explicit source-types can be identified using welldefined area or line-source configurations. The CALGRID (Scire et al., 1989) dispersion model is used for wind-blown dust emissions where the entire model domain is a potential emission source.

A diagnostic meteorological model, called the CALifornia METeorological (CALMET) model (Scire et al., 2000b) is also integrated in DUSTRAN. The main function of CALMET is to create gridded fields of wind and boundary-layer parameters from observed meteorological data. These gridded fields are then supplied to the CALPUFF and CALGRID dispersion models, which perform the plume advection, diffusion, and deposition calculations. Figure 2.1 shows the linkages of these dust dispersion models within DUSTRAN.

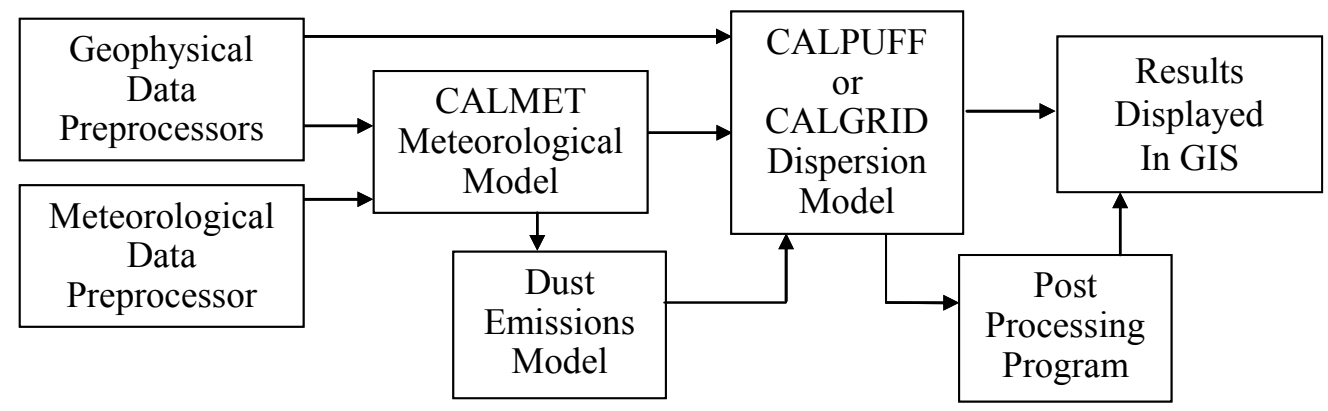

Figure 2.1. Dispersion Model Components within the DUSTRAN Modeling System 
Numerous data preprocessors interface CALMET, CALPUFF, and CALGRID to routinely available terrain elevation and land-use datasets for use in model calculations. A post-processing program is also available for computing average concentration and deposition values. All the model components are dynamically linked by the DUSTRAN interface. Table 2.1 lists the version numbers for the model components as implemented in DUSTRAN 1.0.

Table 2.1. Version Numbers for Model Components Implemented in DUSTRAN 1.0

\begin{tabular}{|l|l|c||}
\hline \multicolumn{1}{|c|}{ Component } & \multicolumn{1}{|c|}{ Purpose } & $\begin{array}{c}\text { Version } \\
\text { Number }\end{array}$ \\
\hline CALPUFF & Dispersion model & 5.5 \\
\hline CALGRID & Dispersion Model & 1.6 \\
\hline CALMET & Meteorological Model & 5.2 \\
\hline CALPOST & Post-processing Program & 5.2 \\
\hline TERREL & Terrain Preprocessor & 2.1 \\
\hline CTGPROC & Land Use Preprocessor & 1.2 \\
\hline MAKEGEO & Merges Terrain/Land Use Datasets & 1.1 \\
\hline READ62 & $\begin{array}{l}\text { Meteorological Preprocessor for Extracting Standard } \\
\text { Upper Air Formats }\end{array}$ \\
\hline
\end{tabular}

The following sections provide a brief technical overview of the DUSTRAN model components. Numerous documents (e.g., Scire et al. 1989; Scire et al. 2000a; Scire et al. 2000b) discuss the theoretical and technical basis of the dispersion and meteorological models used within DUSTRAN; readers are referred to these documents for detailed information on CALPUFF, CALGRID, and CALMET. A cursory overview is provided here of these model components and their integration within the DUSTRAN framework. A more detailed discussion of the vehicular and wind-blown dust-emissions factor module is given in Section 2.5, which is the technical documentation and reference for this component.

\subsection{CALMET}

CALMET is a diagnostic meteorological model that generates three-dimensional gridded wind fields and two-dimensional fields of boundary-layer parameters. Surface and upper-air meteorological observations are required to generate the gridded fields. These data are supplied through the "Meteorology" tab within DUSTRAN (see Section 4.8).

Table 2.2 lists required meteorological observations used by CALMET. The surface data are hourly observations whereas the upper-air vertical profiles are required less frequently, normally twice daily (00Z and 12Z). Multiple surface and upper-air stations may be used, and the stations are not required to be on the DUSTRAN domain. CALMET can interpolate the data to the domain. These input meteorological data are written to formatted surface and upper-air files by the DUSTRAN interface for use in CALMET. 
Table 2.2. CALMET Meteorological Input Requirements

\begin{tabular}{||l|l||}
\hline Surface Data (Hourly) & Upper Air Data (Twice-daily) \\
\hline Wind Speed and Direction & Wind Speed and Direction \\
Temperature & Temperature \\
Cloud Cover & Pressure \\
Ceiling Height & Elevation \\
Surface Pressure & \\
Relative Humidity & \\
\hline
\end{tabular}

Geophysical data are also used by CALMET to derive the gridded meteorological fields. These data (terrain elevations and land-use/land-cover) are routinely available in datasets from the U.S. Geological Survey with varying spatial resolution. In DUSTRAN, terrain data are supplied through GTOPO30 files, which are digital elevation models (DEMs) with a horizontal spacing of 30 arc seconds (approximately 1 kilometer). Land-use/land-cover data are supplied through global land cover characteristics files (GLCC) and are of similar resolution.

Preprocessing programs interface the geophysical datasets with the CALMET meteorological model. These preprocessors, shown in Figure 2.2, are implemented within DUSTRAN to automatically extract the required geophysical data based on the user's domain size. The extracted data are used in the CALMET model formulations and are also written to the CALMET output file for use in the CALPUFF and CALGRID dispersion models.

The procedures that CALMET uses to derive the gridded meteorological fields are controlled largely by an input file called "Calmet.inp." The input file is a text file with a series of keywords that are logically grouped based upon their overall function within CALMET. Every site in DUSTRAN has a "StaticData" directory that stores the template Calmet.inp to be used for that site. The template file is merged with user-input from the DUSTRAN interface before running the model. The parameter settings within the template file are set to optimized values to produce the most realistic output (see Appendix A.2.2). Caution should be exercised if the user wishes to change any setting within the template file, as unrealistic results may be produced.

The following subsections provide an overview of the CALMET procedures for deriving gridded meteorological fields using the meteorological and geophysical input datasets defined previously. Technical formulations are not provided here, as they are available in the CALMET User's Guide (Scire et al. 2000b); instead, the CALMET processing and creation of gridded meteorological fields are described qualitatively.

\subsubsection{CALMET-derived Wind Field}

CALMET uses a two-step process to create the three-dimensional wind field for each hourly time step. In step one, an "initial guess" wind field is modified for terrain effects. In step two, surface and upper-air observations are merged objectively with the step-one, terrain-adjusted winds to create the final flow field. Each step is briefly discussed below. 


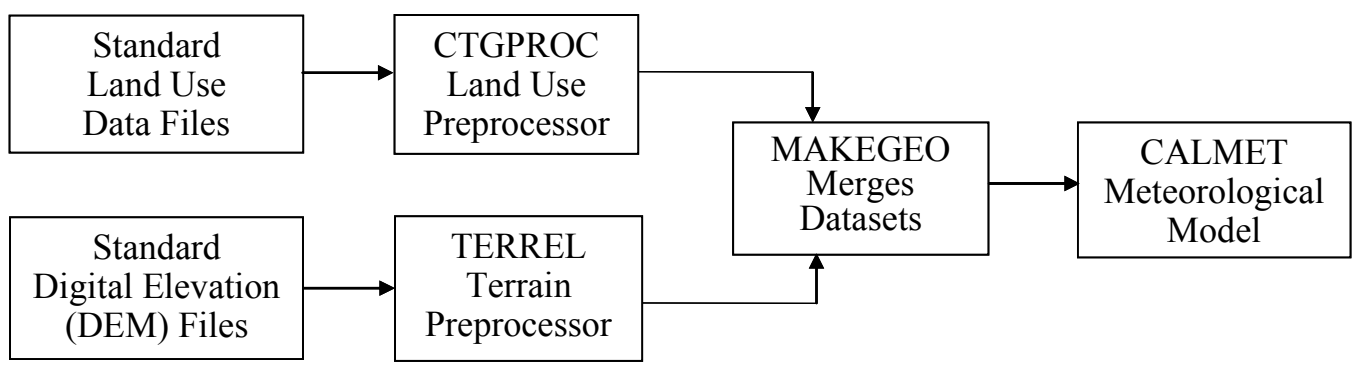

Figure 2.2. Data Flow and Geophysical Preprocessors for CALMET

\subsubsection{Step-One Wind Field Formulation}

The step-one wind field formulation begins with an "initial guess" wind field. The initial guess field can be a spatially varying or a constant, domain-mean wind used throughout the grid. In DUSTRAN's implementation of CALMET, the initial guess wind field is spatially varying and is based on surface and upper-air observations. The surface observations are extrapolated vertically using a power-law or MoninObukhov (M-O) similarity theory, assuming a neutral boundary layer, with M-O extrapolation used by default. The vertically extrapolated surface winds are then merged with the upper-air observations at each node on the grid using a $1 / \mathrm{r}^{2}$ interpolation. During the merging, a bias can be applied at each vertical level in the domain, whereby the relative weighting of the surface and upper-air data can be controlled. This level-by-level bias allows for surface data to more greatly influence the flow field in the lowest layers and the upper-air data to dominate the higher layers.

Once the initial guess field has been created, it is adjusted for terrain effects. CALMET has the option of adjusting the wind field for kinematic effects, slope flow, and flow blocking. Each option can be explicitly treated, and the cumulative effects are merged with the initial-guess field to determine the step-one flow field.

1. Kinematic effects are calculated by assuming an initial zero vertical velocity in the first-guess wind field. The vertical velocity is then calculated because of topographic effects, and the horizontal velocity is adjusted using a divergence minimization scheme that iteratively adjusts the horizontal wind components until the three-dimensional divergence is less than a specified value.

2. Slope flow effects, such as upslope flows during the day or drainage flows at night, are based on an empirical scheme that is a function of the terrain slope, distance to the crest, and the sensible heat flux. A separate formulation for the sensible heat flux is used for the daytime and nighttime in CALMET and is performed for overland locations only.

3. Flow blocking, which is the result of stable stratification, is determined by calculating the Froude number for each grid node in CALMET. If a critical Froude number is not exceeded, then the flow is blocked by terrain and is adjusted tangent to the land feature (i.e., the flow is forced around the land feature).

Of the three terrain adjustment procedures, the kinematic effects can sometimes lead to unrealistically large horizontal velocities, particularly in complex terrain. Therefore, the kinematic adjustment is not implemented in DUSTRAN. 


\subsubsection{Step-Two Wind Field Formulation}

The step-two formulation is an objective merging of the terrain-adjusted, step-one wind field with surface and upper-air observations. The objective analysis is performed level-by-level by first extrapolating the surface wind observations vertically using a constant power-law or M-O as a function of stability. Then, for a given level, observations that are within a specified radius are weighted equally with the step-one wind field. All other observations at that level have a $1 / \mathrm{r}^{2}$ weighting out to a specified radius of inclusion. The radii for equal weighting and inclusion can be specified separately for the surface and all other vertical levels.

Each level of the merged wind field is then smoothed, and the divergence at each grid cell is calculated to provide a new estimate of the vertical velocity. The vertical velocity for the top level of the domain can be set to zero (called the O'Brian adjustment procedure), and the horizontal wind components are readjusted to be mass consistent with the new vertical velocity field using a divergence minimization procedure. The resulting wind field is the final wind field that is output by CALMET for use in the CALPUFF or CALGRID dispersion calculations.

\subsubsection{CALMET-Derived Boundary Layer Parameters}

CALMET contains a micrometeorological model that is based upon an energy balance method, whereby the sensible heat flux is calculated at each grid node by parameterizing the unknown termslatent heat flux, anthropogenic heat flux, ground storage/soil heat flux, and net radiation - in the surface energy balance equation. Once the sensible heat flux is calculated, gridded fields of other boundary layer parameters that are functionally dependent on the sensible heat flux, such as the M-O length and surface friction velocity, are computed.

CALMET has various formulations for calculating the mixing height and are based upon time of day and stability classification. For unstable, daytime conditions, the mixing height is thermally driven, and so it is a function of the surface heat flux and the vertical temperature profile from upper-air soundings. For stable, nighttime conditions, the mixing height is mechanically driven, and so it is functionally dependent upon the friction velocity.

Because of the explicit use of the surface energy balance method in CALMET, all DUSTRAN simulation start times must start after midnight and before sunrise. CALMET contains time validation routines that mandate a start time of $5 \mathrm{a} . \mathrm{m}$. local time, or earlier. So, if a noon-time or evening release is desired, the simulation start time must begin by 5 a.m. even though the source release time may not occur until much later in the day. Normally, this is of little consequence, as the model runtime is extremely fast and efficient.

\subsubsection{Meteorological Data Input Options}

DUSTRAN allows the user to specify four sources of meteorological data to be used by CALMET (see Section 4.8). The four options are:

1. Available Data: Use available site-specific meteorological data where data format is known by DUSTRAN and data ingest utilities are available in DUSTRAN. Currently, this feature applies to data from the DOE's Hanford operations meteorological network (within DUSTRANs "Yakima" site; included with the DUSTRAN install) and data from the DoD's Fort Irwin national training center meteorological network (within DUSTRANs "Ft. Irwin" site; not included with install). 
This is a special option that requires modification to the DUSTRAN code for implementation at other sites. Any user wishing to use meteorological data from special networks will need to contact PNNL for modifying DUSTRAN to read the specific data format. Another option for using site-specific meteorological data in DUSTRAN is to use CALMET utilities described in CALMET documentation to produce files in the format read directly by CALMET. These meteorological data can then be used by DUSTRAN with option 3 User Defined described below.

2. Single Observation: Use single-point meteorological observations specified in DUSTRAN through an input window. DUSTRAN creates one "surface" data file and one "upper-air" data file from the user input in the format needed by CALMET. The "dummy" stations are located at the center of the modeling domain and are assumed to persist for the duration of the simulation.

3. User Defined: Use surface and upper-air meteorological data files (surf.dat and up_1.dat, up_2.dat,... up_n.dat) that have already been prepared for being directly read by CALMET. These files are created outside of DUSTRAN using CALMET utilities.

4. National Oceanic \& Atmospheric Administration (NOAA) Archived: Use meteorological data archived from web-site-accessible National Weather Service (NWS) surface and upper-air data stations. (Note: To use this option, the meteorological data archive application-MetArchivermust be running to populate the observations database. See Section 5.1 for more information).

Of the four methods, option two, "Single Observation," is the only method that relies on user input for defining basic meteorological conditions. These inputs are then used by DUSTRAN to construct all required inputs for use in the CALMET model. The other options (1,3, and 4) are actual data streams coming from defined sources. The methodology used within DUSTRAN to construct the necessary meteorological inputs for CALMET when using "Single Observation" is described in the next section.

\subsubsection{Single Observation Methodology}

The "Single Observation" option provides the user with a very easy and convenient way to quickly view the effects of various configurations (e.g., multiple sources, long-rang transport, nighttime stable flows) on resulting concentration and deposition fields. Even though the single-point meteorological observation persists and is used for the entire simulation, the model-derived meteorological grids will still vary spatially and temporally, as they are a function of land use, topography, and the surface-sensible heat flux (i.e., time of day). CALMET contains a solar model for use in determining sensible heat flux (which drives diffusion rates and mixing height growth) as a function time.

The user specifies wind speed, wind direction, mixing height, ambient temperature, relative humidity, ambient pressure, and atmospheric stability through the DUSTRAN meteorological input window (see Section 4.8.2). CALMET also needs other surface quantities for completeness. These are ceiling height, opaque sky cover, and precipitation code, which are specified near the start of the cal.par DUSTRAN setup file (see Section A.2.1). The cal.par is a static text file that is used to initialize certain parameters in DUSTRAN. The file can be edited in a standard text editor. However, because it allows many features of DUSTRAN to be controlled, caution should be exercised if modifications to this file are desired. The default values in cal.par for ceiling height, opaque sky cover, and precipitation code are 100 (units are hundreds of feet), 0 (units are tenths of coverage), and 0 (no precipitation), respectively. The default values for the meteorological variables specified through the DUSTRAN user-input window are also given in the cal.par file. 
CALMET requires at least one upper-air sounding for operation. Using the single observation data entered by the user and parameters listed in the cal.par file, DUSTRAN automatically generates an upperair sounding file, which is used by the CALMET model. The sounding data consist of pressure, temperature, wind speed, and wind direction at several heights where the lower and upper heights and the number of heights are specified in the cal.par file (see Section A.2.1). The height spacing is logarithmic to allow narrower spacing close to the surface. For simplicity, the wind direction is assumed constant with height and the wind speed is assumed to increase with height using the power law relationship

$$
U_{n}=U_{1}\left(\frac{Z_{n}}{Z_{1}}\right)^{P}
$$

where $U_{n}=$ wind speed at sounding height " $n$ " $\left(\mathrm{m} \mathrm{s}^{-1}\right)$

$\mathrm{U}_{1}=$ wind speed at lowest sounding height $\left(\mathrm{m} \mathrm{s}^{-1}\right)$

$\mathrm{Z}_{\mathrm{n}}=$ sounding height " $\mathrm{n}$ " (m)

$\mathrm{Z}_{1}=$ lowest sounding height $(\mathrm{m})$

$\mathrm{P}=$ power law exponent depending on atmospheric stability.

The power law exponents in Equation 2.1 follow from Turner (1994) as given in his Table 4.6 and listed in the cal.par file (Section A.2.1) for application of DUSTRAN in either "rural" or "urban" areas. The temperature sounding is developed from temperature lapse rates specified as a function of stability in the Cal.par file and the surface temperature specified in the user input window. Consequently, the temperature sounding is determined as:

$$
T_{n}=T_{1}+T_{L R}\left(Z_{n}-Z_{1}\right)
$$

where $T_{n}=$ temperature at sounding height " $n$ " $(K)$

$\mathrm{T}_{1}=$ temperature at lowest sounding height $(\mathrm{K})$

$\mathrm{T}_{\mathrm{LR}}=$ temperature lapse rate depending on stability $\left(\mathrm{deg} \mathrm{m}^{-1}\right)$.

The atmospheric pressure as a function of sounding height is determined from the hydrostatic relationship as:

$$
P_{n}=P_{1} E X P\left[-\frac{a\left(Z_{n}-Z_{1}\right)}{\left(T_{n}+T_{1}\right) / 2}\right]
$$

where $\quad P_{n}=$ pressure at sounding height " $n$ " $(\mathrm{mb})$

$\mathrm{P}_{1}=$ pressure at lowest sounding height $(\mathrm{mb})$

$\mathrm{a}=0.0342 \mathrm{~K} \mathrm{~m}^{-1}$.

\subsection{CALPUFF}

CALPUFF is one of two dispersion models that are implemented in DUSTRAN. The model is ideal for simulating releases from discrete source-type configurations, such as point, line, and area sources, and is activated by setting the "Simulation Type" to "Source Emissions" within the DUSTRAN interface (See 
Section 4.4). The latter two sources - area and line - are integrated with a dust-emissions model and can simulate particle dispersion and deposition from paved or unpaved roadways due to various vehicle types.

As the name implies, CALPUFF is a puff model; it transports and diffuses source material as a series of discrete puffs using gridded meteorological fields from CALMET. The model calculates average plume concentration and deposition flux values at defined receptor locations. The receptor field is automatically defined and created by DUSTRAN based upon the model domain size and source input configuration.

The use of spatially varying meteorological fields makes CALPUFF ideal for medium- and longrange transport applications where domain sizes often exceed 50 kilometers, and the assumption of "spatially homogenous" meteorology used in straight-line plume models often fails. As a result, CALPUFF has gained widespread acceptance and recently has been approved as a regulatory model (40 CFR Part 51, Appendix W) by the EPA for applications involving long-range transport. In DUSTRAN, domain sizes are often on the order of 20 to $400 \mathrm{~km}$; thus, CALPUFF is an appropriate selection for use in the modeling system.

The procedures that CALPUFF uses to define plume transport and dispersion are controlled largely by an input file called "Calpuff.inp." The input file is a text file with a series of keywords that are logically grouped based upon their overall function within CALPUFF. Every site in DUSTRAN has a "StaticData" directory that stores the template Calpuff.inp to be used for that site. The template file is merged with user-input from the DUSTRAN interface before running the model. The parameter settings within the template file are set to optimized values to produce the most realistic output (see Appendix A.2.3).

The sections that follow review some of the more important parameters that are used by CALPUFF to control puff transport and dispersion. Recommendations are made for the various parameter settings and are based upon experience, guidance documents (e.g., Irwin 1998), and CALPUFF's specific implementation within the DUSTRAN system. Extreme caution should be used if the user wishes to change any setting within the template file, as unrealistic results may be produced.

\subsubsection{Near-Field Release Approximation}

Puff models are often computationally restrictive when used for near-field applications involving continuous releases because the puffs are still relatively small, and so enough puffs must be released to approximate the source. In addition, sampling problems may arise near the source if too few puffs are released in a given time-step, especially during rapidly varying meteorological conditions. To address these issues, CALPUFF can use an elongated puff, called a slug, to approximate the release. As the slug is transported downwind and its crosswind dimensions become larger because of dispersion, CALPUFF can transition the slug back to a puff. The slug method is an input parameter set in the CALPUFF input file and is recommended for use in DUSTRAN applications.

\subsubsection{Dispersion Coefficients}

CALPUFF is a Gaussian model and therefore approximates atmospheric diffusion through the specification of dispersion coefficients. The dispersion coefficients are a function of atmospheric stability and affect the vertical and lateral growth of a puff as it is transported downwind. CALPUFF provides many methods for defining the dispersion coefficients, including: 
- Direct measure of the horizontal and vertical velocity variances

- Similarity theory formulations

- Pasquill-Gifford-Turner (PGT) specifications.

Of the listed methods, the similarity theory formulations are recommended in DUSTRAN, as the parameters used in their formulation are explicitly calculated by CALMET.

\subsubsection{Plume Rise}

CALPUFF can account for plume rise, especially from point sources, which are often used to approximate releases from stacks. With stack-type releases, plume buoyancy (due to increased exhaust temperature) and momentum (from exhaust flows) can loft plumes into the air. Plume lofting can result in a phenomenon called "partial plume penetration," whereby part of the plume is ejected into a stable layer (called an inversion) above the release. The overall effect of these parameters is to increase the release height and remove material from the initial plume, all of which act to reduce surface concentration and deposition flux values downwind of the release, particularly near the source. Because DUSTRAN domain sizes tend to be large (e.g., greater than $50 \mathrm{~km}$ ), these effects play a smaller role and only act to increase computation time. They are not recommended for use unless near-field effects are of concern.

\subsubsection{Receptor Grids}

Receptors are locations where the model performs concentration and deposition calculations. In CALPUFF, a primary receptor grid is used for calculating values across the entire domain. The grid is Cartesian and has uniformly spaced receptors in the $\mathrm{X}$ and $\mathrm{Y}$ directions. By default, 50 receptors are specified for both directions, so a 100-km domain, for example, has a receptor spacing of $2 \mathrm{~km}$ in the $\mathrm{X}$ and $\mathrm{Y}$ directions. The number of receptors in the primary grid can be changed within the Cal.par file (see Appendix A.2.1).

In DUSTRAN, secondary receptor grids, or sub-grids, are automatically generated in-and-around sources to increase the resolution of the calculated concentration and/or deposition fields very near the source. These sub-grids are treated as discrete receptors in CALPUFF, and up to 4000 discrete receptors are allowed. For each point source, a polar receptor sub-grid is used. For each area source, a rectangular receptor sub-grid is used. No sub-grid is currently implemented for a line source. The size and resolution of the receptor sub-grids are defined according to parameters set within the Cal.par file (see Appendix A.2.1).

Figure 2.3 is an example of the various receptor grids implemented in DUSTRAN for a CALPUFF

model simulation. Receptors are displayed as blue dots, with the primary Cartesian grid spaced uniformly across the domain and a polar and a rectangular sub-grid centered over their respective source types. 


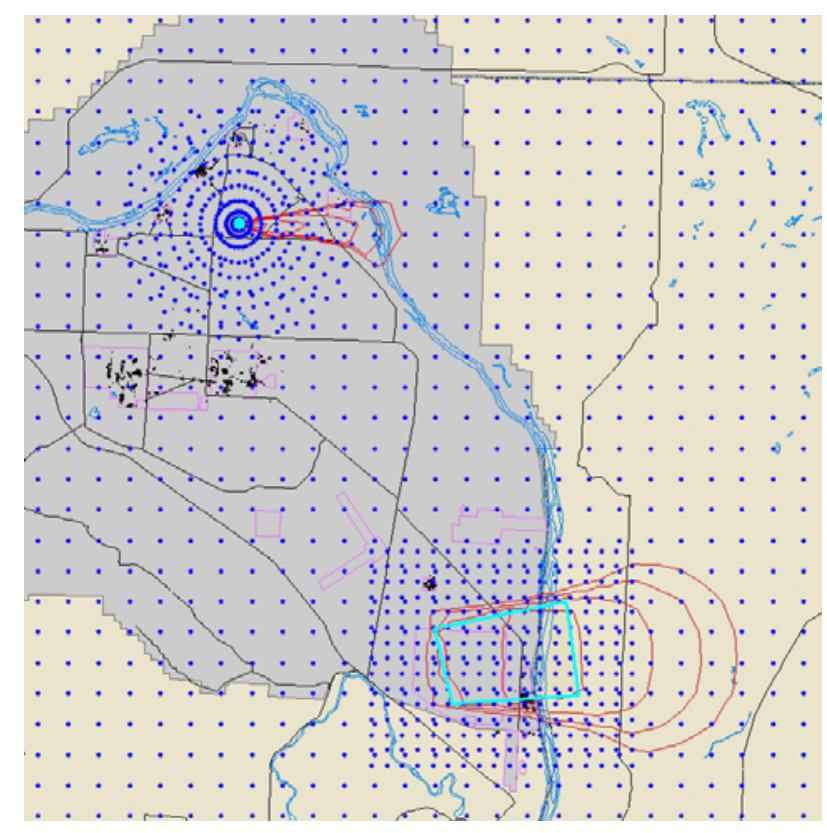

\section{Figure 2.3. Example of the Primary Cartesian Receptor Grid and a Polar and Cartesian Sub-Grid Used Within CALPUFF}

\subsubsection{Representing Moving Vehicles as Line and Area Dust Sources}

DUSTRAN does not treat the motion of individual vehicles, but rather takes a "bulk" approach to dust emissions from vehicle activities. That is, the dust emissions from all vehicles active on a road segment or within a training area over a specified time are assumed to be released uniformly from the road or area at a constant rate throughout the duration of the activity. Therefore, the input fields on the DUSTRAN "Vehicle Parameters" window should not be interpreted as representing the specific motion of individual vehicles, but rather as a convenient approach for providing the vehicle information needed by DUSTRAN. Details of specifying the vehicle characteristics and activities for road segments are given in Section 4.6.2.2 and for training areas are given in Section 4.6.3.2.

As described in Section 2.5.1, the dust emissions from a moving vehicle are proportional to the vehicle momentum (i.e., vehicle weight $\times$ vehicle speed). Therefore, if some vehicles of one type travel at significantly different speeds than other vehicles of the same type, another "vehicle type" will need to be added to the DUSTRAN vehicle list such that the other speed(s) can be specified. Additional vehicles can be specified in DUSTRAN by editing the Cal.par file (see Section A.2.1).

\subsection{CALGRID}

CALGRID is the second dispersion model that is available within DUSTRAN. The model has been implemented to simulate the dispersion of wind-generated dust and is activated by setting the "Simulation Type" to "Wind-blown Dust" within the DUSTRAN interface (See Section 4.4). In the wind-blown dust mode, DUSTRAN creates gridded dust emission factors for the entire model domain, which are then supplied to the CALGRID model to simulate the downwind dispersion and deposition. The dust emission 
factors calculated by DUSTRAN are a function of wind stress, soil texture, and vegetation type across the domain and are discussed further in Section 2.5.2.

CALGRID is a Eulerian model and uses mass continuity to track material throughout a gridded volume. In DUSTRAN, the volume boundaries are defined by specifying a domain in which the user would like to simulate wind-blown dust dispersion. The amount of dust in a given volume is the sum of dust being generated by the wind or lost by deposition as well as the transfer of dust between volumes through wind transport and atmospheric diffusion. The gridded nature of the model makes it ideal for examining releases from large areas, such as wind-blown dust over a large domain.

The CALMET-derived spatially and temporally varying meteorological fields are used in CALGRID to transport and diffuse material throughout the domain. Horizontal transport requires the twodimensional gridded fields of the velocity components ( $U$ and $V$ ) for each vertical layer. Terrainfollowing vertical velocities are used to determine the vertical transport through each of the vertical cell faces in CALGRID. Horizontal diffusion is a function of the CALMET-gridded PGT stability classification, modified for wind speed within each cell and distortion or shear between horizontal cells. Vertical diffusion is calculated from CALMET-gridded similarity fields and is functionally dependent upon the height above ground and stability.

Emissions are introduced into the CALGRID domain depending on the source type. For area sources, which include the model domain for wind-blown dust simulations, emissions are injected into CALGRID using emission layers, with each layer containing a fraction of the total emissions. In DUSTRAN's implementation of CALGRID, area sources have one emission layer, bounded between the surface and 20 meters. For other source types, such as point sources, material is injected into one or more CALGRID layers based on the height of the stack, plume rise due to buoyancy and momentum, and the plume overlap with the model layers.

The procedures that CALGRID uses to define plume transport and dispersion are controlled largely by an input file called "Calgrid.inp." The input file is a text file with a series of keywords that are logically grouped based upon their overall function within CALGRID. Every site in DUSTRAN has a "StaticData" directory that stores the template Calgrid.inp to be used for that site. The template file is merged with user-input from the DUSTRAN interface before running the model. The parameter settings within the template file are set to optimized values to produce the most realistic output (see Appendix A.2.4). Extreme caution should be used if the user wishes to change any setting within the template file, as unrealistic results may be produced.

\subsubsection{Receptor Grid}

In CALGRID, the primary receptor grid is Cartesian and has uniformly spaced nodes in the $\mathrm{X}$ and $\mathrm{Y}$ directions. The nodes serve to both define the horizontal extent of a given cell and specify receptor locations where concentration and deposition values are calculated. Because CALGRID is a Eulerian model, inherent problems exist for situations when the horizontal grid cell size is small and the wind speed is large, as material may be transported through more than one grid cell in a single time step. To minimize this possible issue, 20 nodes in the $\mathrm{X}$ and $\mathrm{Y}$ directions are recommended and are set as the default. Therefore, for a 100-km grid, for example, the cell size (and receptor spacing) is $5 \mathrm{~km}$. The number of nodes in the primary grid can be changed within the Cal.par file (see Appendix A.2.1). 
It should be noted that the outer-band of grid cells in CALGRID serve to initialize the inner grid cells within the domain. These cells are considered "boundary cells" and serve as storage locations for the lateral boundary conditions of the grid; no calculations (e.g., transport, diffusion, deposition) are performed within these cells, and so no values are available for contouring. Therefore, the number of receptors in the $\mathrm{X}$ and $\mathrm{Y}$ directions available for contouring will always be two less than the actual number of nodes.

\subsection{CALPOST}

The CALifornia POST-processing program (CALPOST) is designed to interface with and summarize the output from the CALPUFF or CALGRID models. In DUSTRAN, the CALPOST post-processing module is used to create user-specified time-averaged values from standard hourly outputs generated by the models. In addition, CALPOST is used to create "Top 50" tables, which are tabular values of the highest 50 concentration and deposition values during a simulation for the averaging period of interest. The averaging periods are set within the DUSTRAN interface; currently, 1, 3, 8, and 24 hour averages are available as well as averages calculated for the length of the run. CALPOST can also be run independent of DUSTRAN using results from DUSTRAN, to provide a wider range of output products than accessible through the DUSTRAN interface.

\subsection{Dust-Emission Module}

Dust is injected into the atmosphere through active and natural processes. Active processes primarily involve human activity that directly disturbs the surface - for example, vehicle activity on dirt roads and other unpaved areas or from re-suspension of loose material covering paved roads. Natural processes include wind erosion, which occurs primarily in arid or semiarid environments and may be enhanced by soil disturbance following recent human activity or following natural disasters, such as range fires. The dust-emission module that is incorporated into DUSTRAN accounts for both vehicular and wind-blown dust-generation processes.

\subsubsection{Emission by Vehicular Activity}

The vehicular dust emission module represents dust emissions as the product of an empirically formulated emission factor and the vehicle activity, the latter taken as the total vehicle distance traveled (summed if there are multiple vehicles) in a given period of interest. Explicitly, it can be written as

$$
F_{j}=E_{j} \cdot A
$$

where $\quad F_{j}=$ dust emission due to vehicle activity for particulate size class $j[\mathrm{~g}]$

$E_{j}=$ emission factor for particulate size class $j[\mathrm{~g} / \mathrm{VKT}]$

$A=$ vehicle activity $[\mathrm{VKT}]$

$\mathrm{VKT}=$ vehicle kilometers traveled.

The relations that are used to determine $E_{j}$ are entirely empirical and are usually available for only some of the standard particulate size classes (e.g., $\mathrm{PM}_{2.5}, \mathrm{PM}_{10}, \mathrm{PM}_{15}$, and $\mathrm{PM}_{30}$ ). Variables on which various authors have expressed an $E_{j}$ dependency include the silt content of the surface, the number of vehicle axles, vehicle weight, vehicle speed, and soil moisture. The emission factor, $E_{j}$, is determined as a product of some combination of these variables, each raised to an empirically determined power and a 
fitted constant. The paved- and unpaved-road emission factors in EPA's AP-42 (EPA 2005) are based on this approach and are available for use in DUSTRAN.

Emission factors have been measured for specific vehicles or classes of vehicles. The particulate emission factors for wheeled military vehicles used in DUSTRAN were provided through SERDP research projects. In observations carried out using a variety of wheeled vehicles (primarily military) at Ft. Bliss, Texas, Gillies et al. (2005a, 2005b) found that the only two variables that matter significantly in calculating the $\mathrm{PM}_{10}$ emission factor for unpaved roads are vehicle weight and vehicle speed. Moreover, when weight and speed are properly accounted for, a single empirically derived functional form may be used to calculate a vehicle-specific emission factor. This function may be expressed using

$$
E_{P M 10}=0.003 \cdot W \cdot S
$$

where $W$ is the vehicle weight $(\mathrm{kg})$, and $S$ is the mean vehicle speed $(\mathrm{km} / \mathrm{h})$.

Combining Equations 2.4 and 2.5 and summing over the types of vehicles operating on an unpaved road of length, $L$, during time period, $T$, gives the total emission from the road for that time period as:

$$
F_{P M 10}=0.003 \cdot \sum_{i=1}^{k} W_{i} \cdot S_{i} \cdot A_{i}
$$

where $\quad i=$ vehicle type (e.g., Humvee; specifies vehicle weight)

$k=$ total number of vehicle types

$A_{i}=L \cdot N_{i}$

$N_{i}=$ total number of vehicles of type $i$.

The DUSTRAN vehicle-activity dust-emission module produces total emissions for each road segment over the time period, $T$. Vehicular dust emissions are then passed to the CALPUFF dispersion model where they are released into the modeling domain uniformly along each road segment in both space and time for the duration of the activity.

The vehicle emissions module requires the Universal Transverse Mercator (UTM) easting and northing coordinates to describe the starting and ending points of each road segment as well as the activity duration. Within DUSTRAN, the roadways are created graphically by drawing each segment within the ArcMap map window. For a given line segment, DUSTRAN prompts the user to enter the weight and mean speed for various vehicle types traveling on the roadway. For paved surfaces, emission factors are based on EPA AP-42 recommended values (EPA 2005) and are available for $\mathrm{PM}_{2.5}, \mathrm{PM}_{10}$, $\mathrm{PM}_{15}$, and $\mathrm{PM}_{30}$. For unpaved road surfaces, the user has the option of specifying whether to use emission factors derived from EPA AP-42 (EPA 2005) or from Gillies et al. (2005a, 2005b). Because the Gillies et al. (2005a, 2005b) work is specifically for $E_{\mathrm{PM} 10}$, emission factors for other size classes under this option are estimated by computing the ratio of Gillies et al. $E_{\mathrm{PM} 10}$ to EPA AP-42 $E_{\mathrm{PM} 10}$ and applying this ratio to values for EPA AP-42 unpaved road $E_{\mathrm{PM} 2.5}$ and $E_{\mathrm{PM} 30}$ particle class sizes. As of this writing, AP-42 does not include recommendations for unpaved road $\mathrm{PM}_{15}$ emission factors; $\mathrm{PM}_{15}$ emissions under both options are thus estimated via a linear interpolation between $\mathrm{PM}_{10}$ and $\mathrm{PM}_{30}$ emissions. Average 
fleet weight and average fleet speed, required for AP-42 formulations, are calculated automatically from information input by the user in the DUSTRAN interface.

During military training exercises, off-road activities can occur within specific training areas where numerous vehicles can move around the area (both on-road and off-road) during a period of time where the specific paths of the vehicles are not known. DUSTRAN treats this area-wide training activity as an area source (see Section 4.6.3.1 - Setting Area-Source Release Parameters). The total area-wide dust emissions for each particle size range during the period of the training are determined using the same method as for roads described above. Knowing the total distance traveled by each vehicle type during the training period, the total dust emissions for each particle size range are determined using the emission factors described above for unpaved roads. No distinction is made between dust emissions from vehicles operating on unpaved roads versus vehicles operating during off-road maneuvers. At this time, dustemissions factors for off-road activities are not available; and the assumption is made that emission factors for unpaved roads are a reasonable surrogate to off-road vehicular activities.

\subsubsection{Windblown Dust}

The windblown dust formulation in DUSTRAN provides a measure of the dust emission from the modeling domain caused by wind erosion of the surface. These emissions are a function of the surface wind stress, vegetation class, and soil texture across the modeling domain. The surface wind stress, as approximated by the friction velocity, is calculated as a function of time and location from the CALMET meteorological model. Vegetation class and soil texture coverage are obtained from well-established global databases and are discussed further in Sections 2.5.2.3 and 2.5.2.4, respectively.

The "Add Site Wizard" (Section 6.0) within DUSTRAN automatically creates vegetation class and soil texture files for use in a wind-blown dust simulation whenever a new site is created. These characteristic files, which are a subset of the original global datasets, can be used for any domain specified within a site and are the default files that are used for generating dust emissions in a wind-blown dust simulation. Additionally, the user has the option of specifying finer-resolution characteristic files (see Section 4.6.9) and these files can be ranked in their order of use in a given simulation. When used in this way, high-resolution files can provide detailed information in user-specific regions within the domain and the default files provide information where there is no user-specified information. High-resolution files can be created using the "Polygon Layer Creator Utility" (see Section 5.2), which is an easy way to build vegetation class and soil texture files or for exploring the effects of vegetation removal or soil disruption (e.g., off-road vehicle traffic disturbing soils in new areas, field plowing, etc.) on dust emissions.

The approach in DUSTRAN for computing $\mathrm{PM}_{10}$ concentrations resulting from wind-blown dust is to first calculate gridded fields of wind-generated dust emissions over the modeling domain for each model time step. This time- and space-varying dust emission data are then provided to the CALGRID dispersion model, which uses winds from CALMET for transporting, dispersing, and depositing the emitted dust throughout the modeling domain. The wind-blown dust emissions for each model grid cell are calculated using the method given in the following sections (2.5.2.1 through 2.5.2.4). The principal information needed to calculate the dust emissions for each model grid cell is the time-varying friction velocity (from CALMET), the area-weighted average vegetation mask, and the area-weighted average fraction of total dust emissions by particle size category for each grid cell. Section 2.5.2.5 gives the method for calculating the fractional distribution of vegetation class and soil texture for each grid cell. 


\subsubsection{Dust Flux as a Function of Friction Velocity}

Numerous authors over the past three decades have made laboratory and field measurements of dust flux from wind erosion and empirically related those measurements to the friction velocity, $u_{*}$, which is a measure of wind stress on the surface. There have been some efforts to provide a theoretical foundation for the functional form of the flux in terms of friction velocity, but observations continue to have a great deal of scatter and do not yet validate particular theoretical results. The primary difference among published relations is whether the flux depends on $u_{*}$ raised to the third or fourth power. Because of their field measurements of dust flux, $\mathrm{G}\left(\mathrm{g} \mathrm{cm}^{-2} \mathrm{~s}^{-1}\right)$, under a variety of conditions, we have adopted the formulation of Gillette and Passi (1988):

$$
G=C u_{*}^{4}\left(1-\frac{u_{*_{t}}}{u_{*}}\right)
$$

where $u_{* t}\left(\mathrm{~cm} \mathrm{~s}^{-1}\right)$ is a threshold friction velocity below which dust emission does not occur. In addition to the uncertainty in the exponent of $u_{*}$, there has also been significant experimental variation in the values of $u_{*}$.

Gillette and Passi did not actually publish values of $\mathrm{C}$ and $u_{* t}$ in the above relation. However, they did graphically present a variety of observations of $G$ versus $u_{*}$. We digitized the data from their graph and computed the root-mean-square (rms) difference between the function above and the data for a variety of combinations of $\mathrm{C}$ and $u_{*_{t}}$. These results are shown as a contour plot in Figure 2.4. The figure shows that there is a broad region over which the rms differences are not much different from the absolute minimum value, which occurs near a fitted threshold friction velocity of about $33 \mathrm{~cm} \mathrm{~s}^{-1}$. We selected compromise values of $C=1.0 \times 10^{-14} \mathrm{~g} \mathrm{~cm}^{-6} \mathrm{~s}^{3}$ and $u_{*_{t}}=20 \mathrm{~cm} \mathrm{~s}^{-1}$, which places the threshold friction velocity below the lowest reported value of $u_{*}$, because some of the observations of Gillette and Passi showed dust flux occurring for values of $u_{*}<30 \mathrm{~cm} \mathrm{~s}^{-1}$. Figure 2.5 shows the fit with our coefficients. 


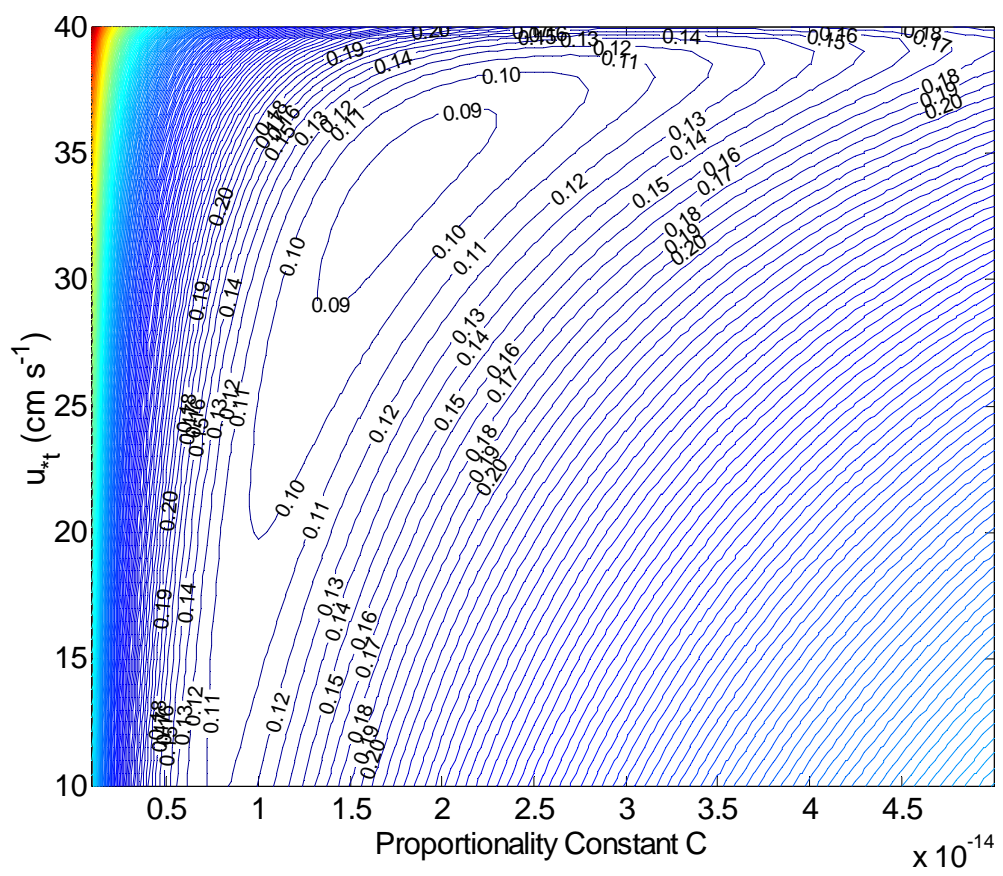

Figure 2.4. Contour Plot of RMS Differences Between Equation (2.7) and Observations of Dust Flux G Discussed by Gillette and Passi (1988)

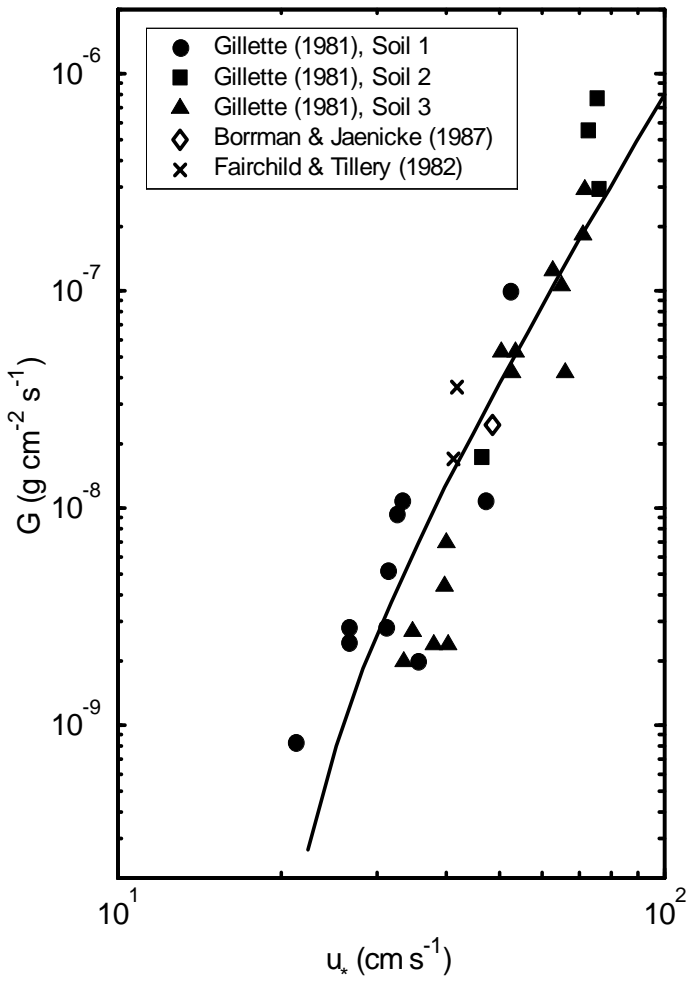

Figure 2.5. Observations of $G$ Versus $u^{*}$ after Gillette and Passi (1988).

The solid line is Equation (2.7) with $C=1.0 \times 10^{-14} \mathrm{~g} \mathrm{~cm}^{-6} \mathrm{~s}^{3}$ and $u_{* t}=20 \mathrm{~cm} \mathrm{~s}^{-1}$. 


\subsubsection{Effect of Soil Moisture}

Soil moisture, if a measure is available, is taken into account in our approach using the method of Fecan et al. (1999) as cited by Nickovic et al. (2001). The soil moisture is incorporated through a wetness factor, $f_{w}$, that multiplies the threshold friction velocity to increase it. Consequently, the total dust flux from the surface accounting for soil moisture is

$$
G=C u_{*}^{4}\left(1-\frac{f_{w} u_{* t}}{u_{*}}\right)
$$

where the soil wetness factor is given as

$$
f_{w}=\left\{\begin{array}{cc}
{\left[1+1.21\left(w-w^{\prime}\right)^{0.68}\right]^{1 / 2}} & w>w^{\prime} \\
1 & w \leq w^{\prime}
\end{array}\right.
$$

$w$ is the gravimetric soil moisture (mass of water/mass of soil; \%), and $w^{\prime}$ is the maximum amount of water that can be adsorbed by the soil (\%), given as a function of the fraction of clay in the soil, $\beta_{1}$ (see Section 2.5.2.4). $w^{\prime}$ is given as

$$
w^{\prime}=14 \beta_{1}^{2}+17 \beta_{1}
$$

At this time, soil moisture, $w$, is not a function of time or location in DUSTRAN, but is currently specified as a constant value in the Cal.par model static file described in Section A.2.1.1. The default value is zero, leading to a wetness factor of one, which is dry soil.

\subsubsection{Vegetation Cover Effects on Dust-Emitting Potential}

Equation 2.8 gives the maximum wind-generated dust flux from the surface not accounting for the effects of different types of vegetation cover on dust-emitting potential. Essentially, more vegetation cover results in less wind-blown dust generated from the surface. This effect of vegetation cover on wind-blown dust is treated by simply multiplying the dust flux from Equation 2.8 by a vegetation mask, $\alpha$, that ranges from zero to one. The total dust flux from the surface accounting for vegetation is then given as

$$
G=\alpha C u_{*}^{4}\left(1-\frac{f_{w} u_{* t}}{u_{*}}\right)
$$

The vegetation mask is determined using the Olson World Ecosystem (Olson, 1992) that defines 59 distinct classes of vegetation. Of the 59 classes, only four have been sufficiently exposed soil to allow for wind erosion, and they include two desert categories and two semi-desert categories. Because of the sparseness of vegetation in deserts, $\alpha$ for those categories has a value of 1.0. Because of the more widespread presence of shrubs and grasses in the semi-desert categories, $\alpha$ is assigned a value of 0.5 . Table 2.3 lists the Olson identification number, vegetation class description, and $\alpha$ for these four classes. 
All other Olson categories are assigned a value of zero for $\alpha$ and therefore do not contribute to windblown dust emissions.

Within DUSTRAN, the default Olson vegetation class file is derived from the original 10-minute resolution global database at the time of the sites creation (see the "Add Site Wizard", Section 6.0). The default dataset can be supplemented with higher-resolution, Olson-based vegetation class files created by the user using the "Polygon Layer Creator Utility" (Section 5.2).

Table 2.3. Olson Vegetation Classes Used in the Wind-blown Dust-Emission Model

\begin{tabular}{||c|l|c|}
\hline \hline ID \# & \multicolumn{1}{|c|}{ Olson Vegetation Class Description } & $\boldsymbol{\alpha}$ \\
\hline 8 & Desert, mostly bare stone, clay and sand & 1.0 \\
\hline 50 & Sand desert, partly blowing dunes & 1.0 \\
\hline 51 & Semi-desert/desert, scrub/sparse grass & 0.5 \\
\hline 52 & Cool/cold shrub, semi-desert/steppe & 0.5 \\
\hline
\end{tabular}

The value of $\alpha$ actually used in Equation 2.11 to calculate the dust emissions for a model grid cell is the area-weighted average of $\alpha$ 's for all the vegetation categories that fall within the grid cell. The areaweighted average vegetation mask, $\bar{\alpha}$, is calculated as

$$
\bar{\alpha}=\sum_{i=1}^{4} f_{i}^{V} \alpha_{i}
$$

where $\alpha_{i}$ is the vegetation mask for the $\mathrm{i}^{\text {th }}$ Olson vegetation class (Table 2.3), and $f_{i}^{V}$ is the area fraction of the $i^{\text {th }}$ Olson vegetation class in a grid cell. The area fractions for the four Olson vegetation classes will sum to one or less. The sum may be less that one because, as noted previously, many other Olson vegetation classes exist that have $\alpha$ 's equal to zero. See Section 2.5.2.5 for the determination of the area fractions.

\subsubsection{Approximating the Size Distribution of Windblown Dust}

The formulation given above estimates the total mass of dust produced by wind. However, it is also important to know the size distribution and in particular how much of the dust consists of particles smaller than $10 \mu \mathrm{m}$ in diameter. To do this, we have used global databases of soil texture class to estimate the fraction of the emitted dust in four separate particle size ranges. Soil textures are typically defined (Tegen and Fung 1994) in terms of their fractions of clay, small silt, large silt, and sand. Table 2.4 gives typical properties of particles for each soil texture class.

The approach for determining the soil texture class (and thus the particle size distribution) follows from Nickovic et al. (2001) using a Zobler soil categories database, and Table 2.5 lists the fractions, $\beta_{j, k}$, of the four (k-index) soil texture classes within each of the seven (j-index) Zobler soil categories. Note that the fractions of soil texture classes for each Zobler category sum to one. It should also be noted that the size fractions for small and large silt given by Nickovic et al. (2001) were too large by a factor of two. The values in Table 2.5 are corrected. 
Table 2.4. Features of Typical Dust Particles (after Nickovic et al. 2001)

\begin{tabular}{||c|l|c|c|c|c||}
\hline \hline $\mathbf{k}$ & $\begin{array}{c}\text { Soil Texture } \\
\text { Class }\end{array}$ & $\begin{array}{c}\text { Range of Particle } \\
\text { Diameters }(\boldsymbol{\mu m})\end{array}$ & $\begin{array}{c}\text { Typical Particle } \\
\text { Diameter }(\boldsymbol{\mu m})\end{array}$ & $\begin{array}{c}\text { Particle Density } \\
\left(\mathbf{g ~ c m}^{-3}\right)\end{array}$ & $\boldsymbol{\gamma}_{\boldsymbol{k}}$ \\
\hline 1 & clay & $1-2$ & 1.5 & 2.50 & 0.08 \\
\hline 2 & small silt & $2-20$ & 12 & 2.65 & 1.00 \\
\hline 3 & large silt & $20-50$ & 36 & 2.65 & 1.00 \\
\hline 4 & sand & $50-100$ & 76 & 2.65 & 0.12 \\
\hline
\end{tabular}

Table 2.5. Fractions, $\beta_{j, k}$, of the Soil Texture Classes in each Zobler Soil Category

\begin{tabular}{|c|l|c|c|c|c|}
\hline & \multirow{2}{*}{$\begin{array}{c}\text { Zobler Soil } \\
\text { Categories }\end{array}$} & $\mathbf{k}=\mathbf{1}$ & $\mathbf{2}$ & $\mathbf{3}$ & $\mathbf{4}$ \\
\cline { 3 - 6 } $\mathbf{j}$ & & $\mathbf{C l a y}$ & Small Silt & Large Silt & Sand \\
\hline 1 & coarse & 0.12 & 0.04 & 0.04 & 0.80 \\
\hline 2 & medium & 0.34 & 0.28 & 0.28 & 0.10 \\
\hline 3 & fine & 0.45 & 0.15 & 0.15 & 0.25 \\
\hline 4 & coarse-medium & 0.12 & 0.09 & 0.09 & 0.70 \\
\hline 5 & coarse-fine & 0.40 & 0.05 & 0.05 & 0.50 \\
\hline 6 & medium-fine & 0.34 & 0.18 & 0.18 & 0.30 \\
\hline 7 & coarse-medium-fine & 0.22 & 0.09 & 0.09 & 0.60 \\
\hline
\end{tabular}

Currently, the soil texture category for a desired location in the modeling domain is being read from an ASCII, comma-delimited text file that was derived from the Zobler raster image with a 1-degree resolution (Staub and Rosenzweig 1992). Sources for higher-resolution soil textures that are spatially complete (e.g., cover the continental U.S.) are being investigated for possible inclusion in a future version of DUSTRAN. Additionally, the user has the option of creating high-resolution Zobler soil texture files using the "Polygon Layer Creator Utility" (see Section 5.2). The user-specific files can be associated with a given simulation (see Section 4.6.9) to supplement or replace the soil textures derived from the default Zobler global file.

To accomplish the size partitioning of the dust flux from each model grid cell, we use the dustproductivity factor as defined by Nickovic et al. (2001). For each of the four particle size classes, we define a dust-productivity factor $\delta_{k}$ so that the dust flux in the $\mathrm{k}^{\text {th }}$ particle size class, $G_{k}$, is

$$
G_{k}=\delta_{k} G
$$

where $\mathrm{G}$ is from Equation 2.11. The dust-productivity factor for a grid cell is determined by

$$
\delta_{k}=\gamma_{k} \sum_{j=1}^{7} f_{j}^{Z} \beta_{j, \boldsymbol{k}}
$$

where $\gamma_{k}$ is the ratio of mass available for uplift to total mass in that soil texture class (size range), $\beta_{j, k}$ is from Table 2.5, and $f_{j}^{Z}$ is the area fraction of the $\mathrm{j}^{\text {th }}$ Zobler soil category in a grid cell. The area 
fractions for the seven Zobler categories sum to one. See Section 2.5.2.5 for the determination of the area fractions. Table 2.4 lists the values of $\gamma_{k}$, which are those used by Nickovic et al. (2001). Because the values of $\delta_{k}$ represent a partitioning of the total flux, G, they should sum to unity. However, the values of G used by Gillette and Passi (1988) to develop Equation 2.7 were for particle sizes smaller than $40 \mu \mathrm{m}$ in diameter. This essentially excludes the larger sand category. This exclusion of the larger sand category is accounted for by actually using an "enhanced" total dust flux, $G^{\prime}$, in Equation 2.13. Therefore, the "actual" dust flux for each particle size (soil texture class) within a grid cell is

$$
G_{k}=\delta_{k} G^{\prime}
$$

where $G^{\prime}$ is determined as

$$
G^{\prime}=\frac{\sum_{k=1}^{4} \delta_{k}}{\sum_{k=1}^{3} \delta_{k}} G
$$

Substituting Equation 2.16 into 2.15 gives the actual dust flux by particle size category as

$$
G_{k}=\delta_{k} \frac{\sum_{i=1}^{4} \delta_{i}}{\sum_{i=1}^{3} \delta_{i}} G
$$

Currently in DUSTRAN, only the $\mathrm{PM}_{10}$ size particles from wind-blown dust are provided as a gridded output. The emission flux of $\mathrm{PM}_{10}$ for each grid cell is the sum of the first two particle size categories, or

$$
G_{P M 10}=\sum_{k=1}^{2} G_{k}
$$

\subsubsection{Calculating Soil and Vegetation Types for Use in Wind-Blown Dust Simulations}

This section provides specific information on how the Olson vegetation class and Zobler soil-texture values are sampled and mapped to model-grid-cell average values in DUSTRAN. Mapping is necessary because the characteristic files provide vegetation and soil texture information as a series of polygon shapes (polygon refers to an area unit within the GIS) whereas CALGRID requires emissions from a regular array of cells within a Cartesian grid. This section is not necessary to understand how windblown dust is determined, but is given here for completeness of the calculations and operations performed within DUSTRAN.

For calculating wind-blown dust emissions, DUSTRAN requires the fractional area coverage of the four Olson vegetation classes and seven Zobler soil texture categories for all the model grid cells within 
the model domain. The fractional area coverage of either the vegetation or soil textures class for each model grid cell is calculated by "sampling" the respective base characteristic file. The default base characteristic files are created automatically at the time of the site's creation (using the "Add Site Wizard", Section 6.0) and are derived from the original Olson World Ecological and Zobler soil textures global database files. The default files are assigned automatically to a given wind-blown dust scenario using the "Sources" tab (see Section 4.6) within DUSTRAN and can be supplemented with higherresolution, user-specific characteristic files created using the "Polygon Layer Creator Utility" (see Section 5.2).

The format of the two base data files includes headers containing the number of data columns and rows found in the two site-wide files as well as headers detailing the UTM coordinates of the lower left and upper right corners of the site extent. Typically, site extents are greater than $600 \mathrm{~km}$ square to accommodate modeling domains centered at various locations and of various sizes. Following the headers are data records containing the soil or vegetation data. Each data record contains five fields separated by commas that represent one grid point in the base file. The first two fields of each record contain the column and row indices of the data's location in the data file. The third element contains the soil or vegetation type ID. A 9999 in the type field represents a grid point that does not contain any soil or vegetation data. Lastly, the fourth and fifth elements are the UTM easting and northing coordinates of the grid point. The $[0,0]$ indices in the file represent the $\mathrm{X}$ and $\mathrm{Y}$ values of the lower left (southwest) corner of the site extent. $\mathrm{X}$ indices increase to the right (east), and $\mathrm{Y}$ indices increase to the top (north).

The fractional area coverage, $f_{k}^{A}$, within a model grid cell for the $\mathrm{k}^{\text {th }}$ category of surface property A (e.g., soil texture) calculates as

$$
f_{k}^{A}=\frac{N_{k}}{N_{G} N_{G}}
$$

where $N_{k}$ is the number of occurrences of the $k^{\text {th }}$ category of property " $A$," and $N_{G}$ is the number of sampling points in the $\mathrm{x}$ - and $\mathrm{y}$-directions in a square model grid cell. Currently in DUSTRAN, $\mathrm{N}_{\mathrm{G}}$ is equal to five for a total of 25 sampling points per model grid cell.

The desired property value for each model grid cell sampling point is read from the appropriate sitewide file containing the specific property (e.g., soil texture, vegetation cover) of interest. The property value at a grid cell point located at $\left[X_{m}, Y_{n}\right]$, where $m=1$ to $N_{G}$ and $n=1$ to $N_{G}$, is determined by knowing the nearest site-wide data point to the sampling point. The indices $\left[i_{m}, j_{n}\right]$ of the nearest sitewide point to the $[\mathrm{m}, \mathrm{n}]$ grid cell point are determined as

$$
\begin{gathered}
i_{m}=N I N T\left[\frac{X_{m}-X_{0}}{X_{d}-X_{0}}\left(N_{x}-1\right)\right] \\
j_{n}=N I N T\left[\frac{Y_{n}-Y_{0}}{Y_{d}-Y_{0}}\left(N_{y}-1\right)\right]
\end{gathered}
$$


where $\quad \mathrm{i}_{\mathrm{m}}=0$ to $\mathrm{N}_{\mathrm{x}}-1 ; \mathrm{X}$-direction index of $\mathrm{m}^{\text {th }}$ grid cell sampling point in site-wide file

$\mathrm{j}_{\mathrm{n}}=0$ to $\mathrm{N}_{\mathrm{y}}-1$; Y-direction index of $\mathrm{n}^{\text {th }}$ grid cell sampling point in site-wide file

$\mathrm{X}_{\mathrm{m}}=$ UTM Easting coordinate of the model grid cell sampling point

$\mathrm{Y}_{\mathrm{n}}=$ UTM Northing coordinate of the model grid cell sampling point

$\mathrm{X}_{0}=\mathrm{UTM}$ Easting coordinate of the southwest corner of the site-wide file

$\mathrm{Y}_{0}=$ UTM Northing coordinate of the southwest corner of the site-wide file

$\mathrm{X}_{\mathrm{d}}=\mathrm{UTM}$ Easting coordinate of the northeast corner of the site-wide file

$\mathrm{Y}_{\mathrm{d}}=\mathrm{UTM}$ Northing coordinate of the northeast corner of the site-wide file

$\mathrm{N}_{\mathrm{x}}=$ Number of data points in the X-direction in the site-wide file

$\mathrm{N}_{\mathrm{y}}=$ Number of data points in the Y-direction in the site-wide file

and NINT means get nearest integer of quantity in brackets.

The $\mathrm{X}_{\mathrm{m}}$ and $\mathrm{Y}_{\mathrm{n}}$ coordinates for each of the grid cell sampling points are determined as

$$
\begin{aligned}
& X_{m}=X_{0}^{G}+\frac{\Delta}{2 N_{G}}[1+2(m-1)] \\
& Y_{n}=Y_{0}^{G}+\frac{\Delta}{2 N_{G}}[1+2(n-1)]
\end{aligned}
$$

where $\Delta=$ Length of square grid cell along one side

$X_{0}^{G}=$ UTM Easting coordinate of the model grid cell southwest corner

$Y_{0}^{G}=$ UTM Northing coordinate of the model grid cell southwest corner

As mentioned previously, the spatial resolution of the Olson vegetation class database is 10 minutes (roughly $15 \mathrm{~km}$ at mid-latitudes) and the Zobler soil texture database is 1 degree (roughly $80 \mathrm{~km}$ at midlatitudes). In general, these data are at a much coarser resolution than the grid resolution that will be likely used in a windblown dust simulation. For example, a $200 \mathrm{~km}$ domain with 20 grid cells in the $\mathrm{X}$ and $\mathrm{Y}$ direction will have a $10 \mathrm{~km}$ resolution, which is a finer resolution than either the default vegetation class or soil texture database files. Nevertheless, the 25 ( 5 by 5) sampling points per grid cell method is not computationally expensive and is employed for situations where the user may supplement a simulation with higher-resolution characteristic files. 


\subsection{DUSTRAN Installation Instructions}

DUSTRAN is implemented within the ArcMap Version 9.x interface and requires that ArcMap be installed before installing DUSTRAN. Also note that the installation of this software requires that the user has administrative privileges on the machine in which it is being installed. Be sure privileges are properly set and that ArcMap is installed before proceeding with DUSTRAN installation. This section provides detailed instructions on:

- Installing DUSTRAN and setting up access to DUSTRAN from within ArcMap

- Installing a sample site, including setting the site's data sources for display within ArcMap.

\subsection{Installing DUSTRAN}

1. Before installing DUSTRAN, be sure all other applications are closed, including ArcMap.

2. Install the DUSTRAN model by double-clicking on the "DUSTRAN.exe" executable file found on the installation CD. Follow the step-by-step installation instructions that are presented by the "InstallShield Wizard."

3. DUSTRAN is accessed through a button on the ArcMap toolbar. To add DUSTRAN to the ArcMap toolbar, launch ArcMap and click on the "Tools" menu and select "Customize" (see Figure 3.1):

\begin{tabular}{|c|c|c|}
\hline \multicolumn{3}{|c|}{ Q FTHood.mxd - ArcMap - ArcView } \\
\hline File Edit View Insert SEelection & Iools Window Help & \\
\hline Editor $\mathbf{V}|\boldsymbol{\wedge}| \boldsymbol{\bullet} \mid$ Task & I. Editor Toolbar & \\
\hline 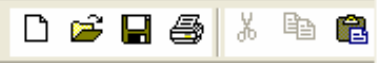 & Graphs & $\bullet$ \\
\hline \multirow{2}{*}{$\square \Theta^{3}$ Layers } & Reports & $\bullet$ \\
\hline & Geocoding & $\bullet$ \\
\hline & $\stackrel{+}{\times} Y+_{Y}^{+}$Add XY Data... & \\
\hline $\begin{array}{l}\square \text { Apgems } \\
+\square \text { Ft. Hood Border } \\
+\square \text { Black-capped Vireo }\end{array}$ & $\frac{\text { It+ }}{+1+}$ Add Route Events... & \\
\hline $\begin{array}{l} \pm \square \text { Black-capped Vireo } \\
\pm \square \text { Golden-cheek Warbler }\end{array}$ & I+1 Buffer Wizard... & \\
\hline \multirow{2}{*}{$\begin{array}{l} \pm \square \text { Lakes } \\
\pm \square \text { Rivers }\end{array}$} & 圆 GeoProcessing Wizard... & \\
\hline & \multicolumn{2}{|c|}{$\pm \square$ Roads ArcCatalog } \\
\hline \multirow{2}{*}{$\begin{array}{l} \pm \square \text { Ft. Hood Training Areas } \\
\pm \square \text { Domains } \\
\pm \square \text { Elevation }\end{array}$} & Macros & • \\
\hline & Customize... & \\
\hline \multirow{3}{*}{$\begin{array}{l} \pm \square \text { Counties } \\
\pm \square \text { PointSources } \\
\pm \square \text { LineSources } \\
\pm \square \text { AreaSources }\end{array}$} & Extensions... & \\
\hline & Styles & • \\
\hline & Options... & \\
\hline
\end{tabular}

Figure 3.1. Customize Option

4. The "Customize" toolbar dialog window will appear (see Figure 3.2). Click on the "Add from file..." button: 


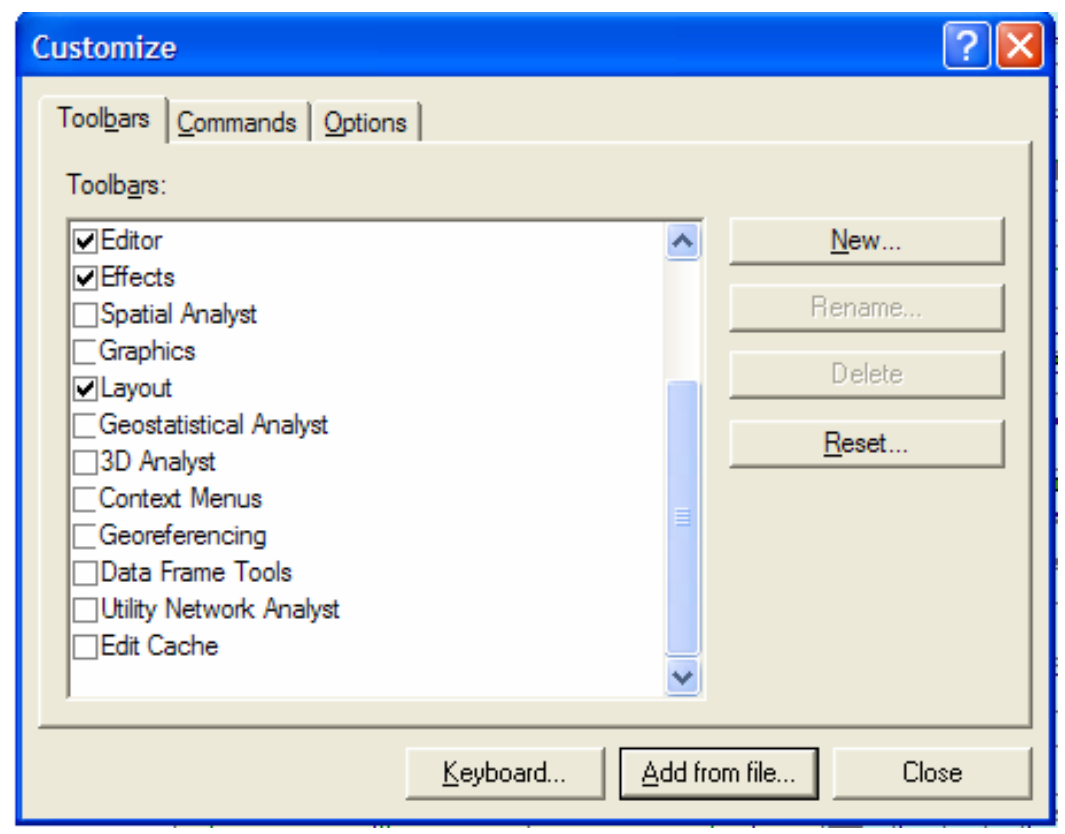

Figure 3.2. "Add from file" Button

5. Browse to the root DUSTRAN directory and select the "DUSTRAN.dll" file and then click "Open" (see Figure 3.3):

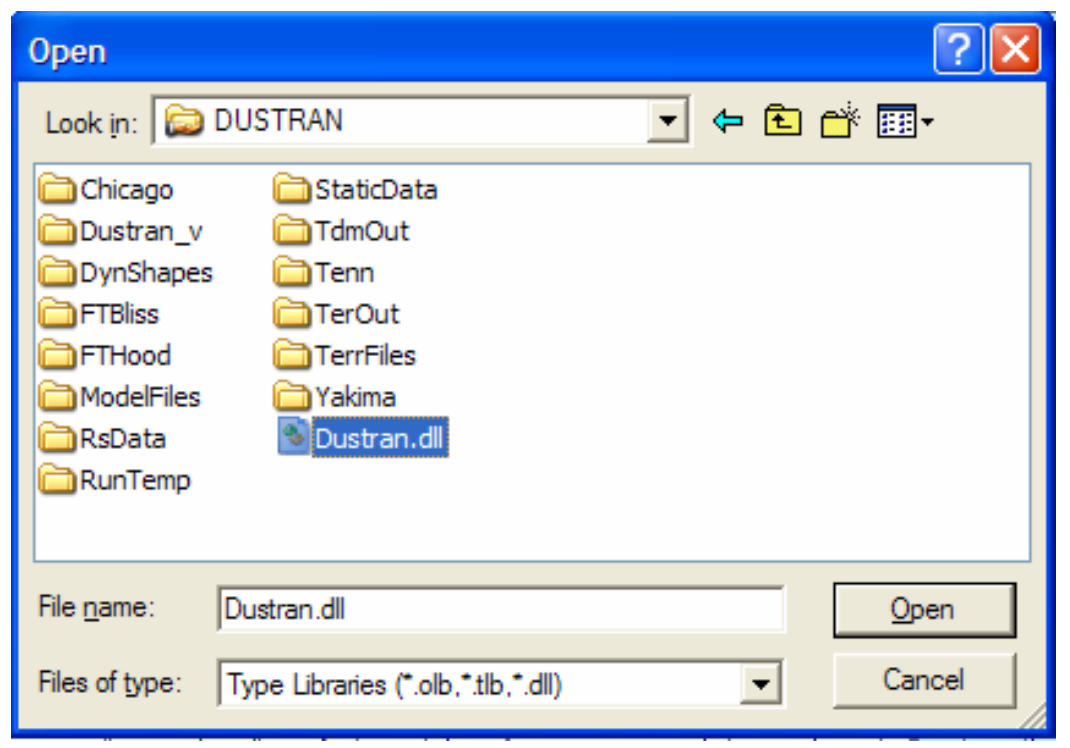

Figure 3.3. "Dustran.dll" Option

A window displaying the objects to be added will be displayed. Close this window by selecting the "OK" button. 
6. In the "Customize" window, check "DUSTRAN" from the list and click the "Close" button (see Figure 3.4).

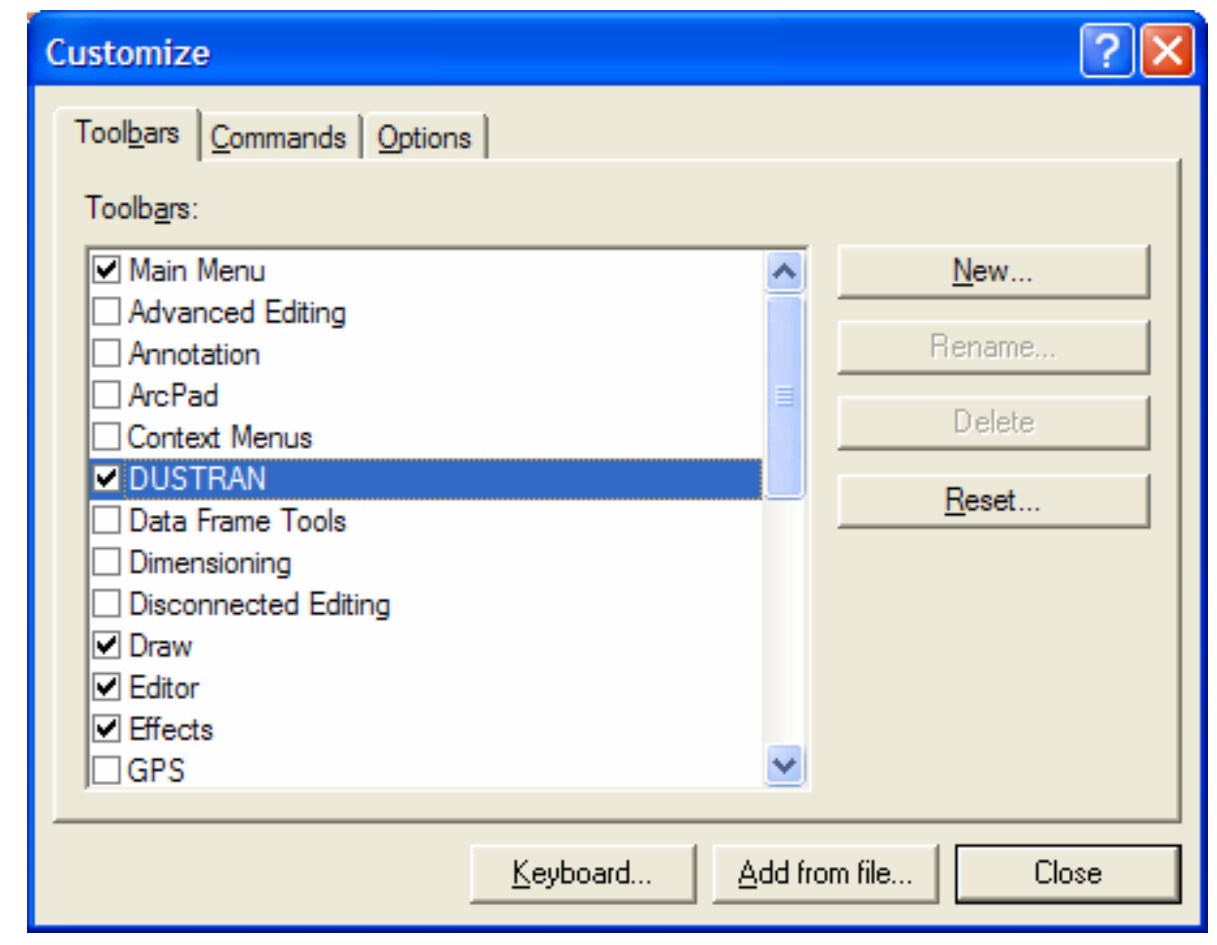

Figure 3.4. "Close" Option

A button called "DUSTRAN," which is used to launch the DUSTRAN model, will appear within the ArcMap interface. (Note: Newly registered tools are often displayed using an image, or icon, by default. It is not uncommon for the new toolbar to appear empty because the DUSTRAN toolbar does not have an associated icon. To correct this setting, right-click on the empty toolbar while the Customize dialog is still open. A context (pop-up) menu will appear with several display options. Select the "text-only" option and type the word "DUSTRAN" into the text box on the context menu.)

7. To allow the button to be dockable within the ArcMap toolbar, close the "Customize" window and shut down the ArcMap application.

8. Using Window's "Explorer" or "My Computer," browse to the directory path "c: $\mid$ arcgis $\backslash$ arcexe90 $\backslash$ bin" and start the "categories" executable by double-clicking on the "categories" icon.

9. Once the "Component Category Manager" window is displayed, scroll down the list and select the "ESRI Mx Dockable Windows" option (see Figure 3.5): 


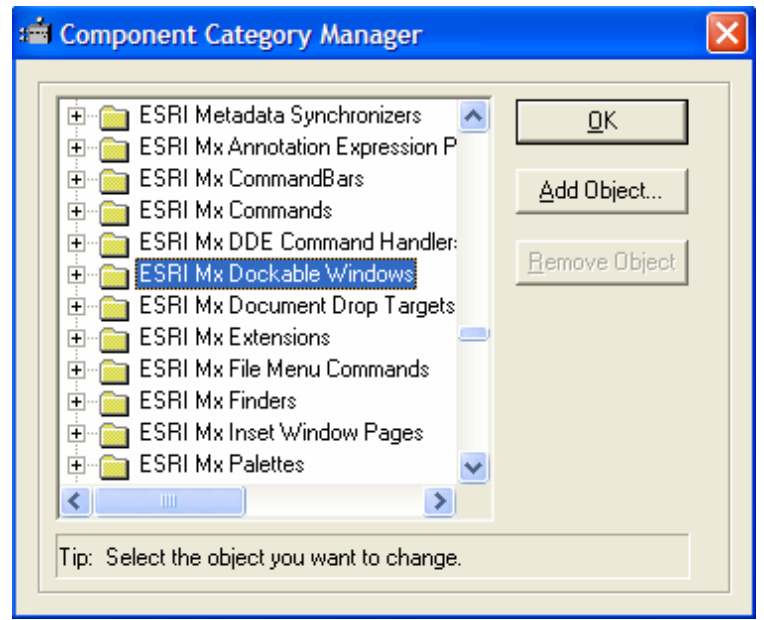

Figure 3.5. "ESRI Mx Dockable Windows” Option

10. Click on the "Add Object" button, and a file selection window will be displayed.

11. Using the file selection window, navigate to the DUSTRAN root directory and select the "DUSTRAN.dll."

12. Once the "Add Objects" window is displayed, select the "Uncheck All" button and check the "DockWin" option from the list of objects (see Figure 3.6):

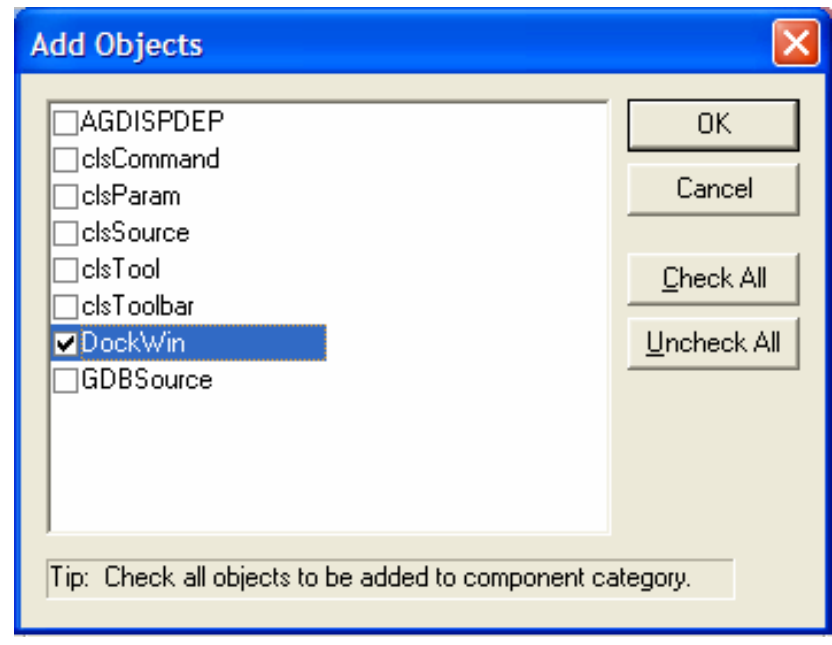

Figure 3.6. "Uncheck All” Option

13. Click "OK" and then click "OK" on the "Component Manager Window" to close the program.

14. Launch ArcMap. A new, detached toolbar button should appear within ArcMap. If the button does not have a title, right-click on the empty toolbar while the Customize dialog is still open. A context (pop-up) menu will appear offering several display options for the DUSTRAN toolbar, including: 
- Text only

- Image only

- $\quad$ Text and Image

Select the "text-only" option. The name of the tool should read "DUSTRAN" by default. If not, type this into the text box on the context menu. Drag the DUSTRAN button to the ArcMap toolbar and click the button to begin the application.

\subsection{Installing a Site}

This section steps through the process of installing a site from the installation CD. The sample site, called Yakima, is included with all DUSTRAN installations. If you are installing a user-specific site, the same procedure will be followed; the only change involves the name of the site.

1. Before installing a site, be sure all other applications are closed, including ArcMap.

2. Example sites are available on the installation CD. These sites contain GIS data layers, terrain data, and land use/land cover information for making DUSTRAN simulations. To install the Yakima site, double-click on the executable file called "Yakima.exe." Follow the step-by-step installation instructions that are presented by the "InstallShield Wizard."

3. After installing the site, launch ArcMap. Within the DUSTRAN interface, click the "Select Site..." button and select the site from the list. To open the Yakima site, select "Yakima" from the list. The site will open in DUSTRAN and is available for performing a simulation. 


\subsection{DUSTRAN User's Guide}

To perform a simulation in DUSTRAN, numerous fields within the interface must be selected, entered, or completed. This section details the various user-entry fields within the interface and is intended as a reference guide for the application. This section assumes that both ArcMap and DUSTRAN are already installed, and the user has some familiarity with ArcMap.

\subsection{Starting DUSTRAN and Loading a Site}

DUSTRAN functions as a console application within ArcMap and is accessible through the ArcMap toolbar (see Section 3.0, "DUSTRAN Installation Instructions"). To start DUSTRAN, first start ArcMap, which immediately shows a window for selecting a map. Leave the window at the default setting of "A new empty map" and click "OK." Then launch DUSTRAN by simply clicking on the "DUSTRAN" button found on the ArcMap toolbar.

Once the DUSTRAN interface has been loaded into ArcMap, click on the "Select Site..." button to open a dialog box that contains a list of available sites. Select a site from the "Current Site" list and click "Open" to open the site in DUSTRAN. The GIS map files associated with the site are automatically loaded and displayed in the ArcMap map window. Figure 4.1 shows the Yakima site loaded into the DUSTRAN modeling system showing the Hanford area of Washington State.

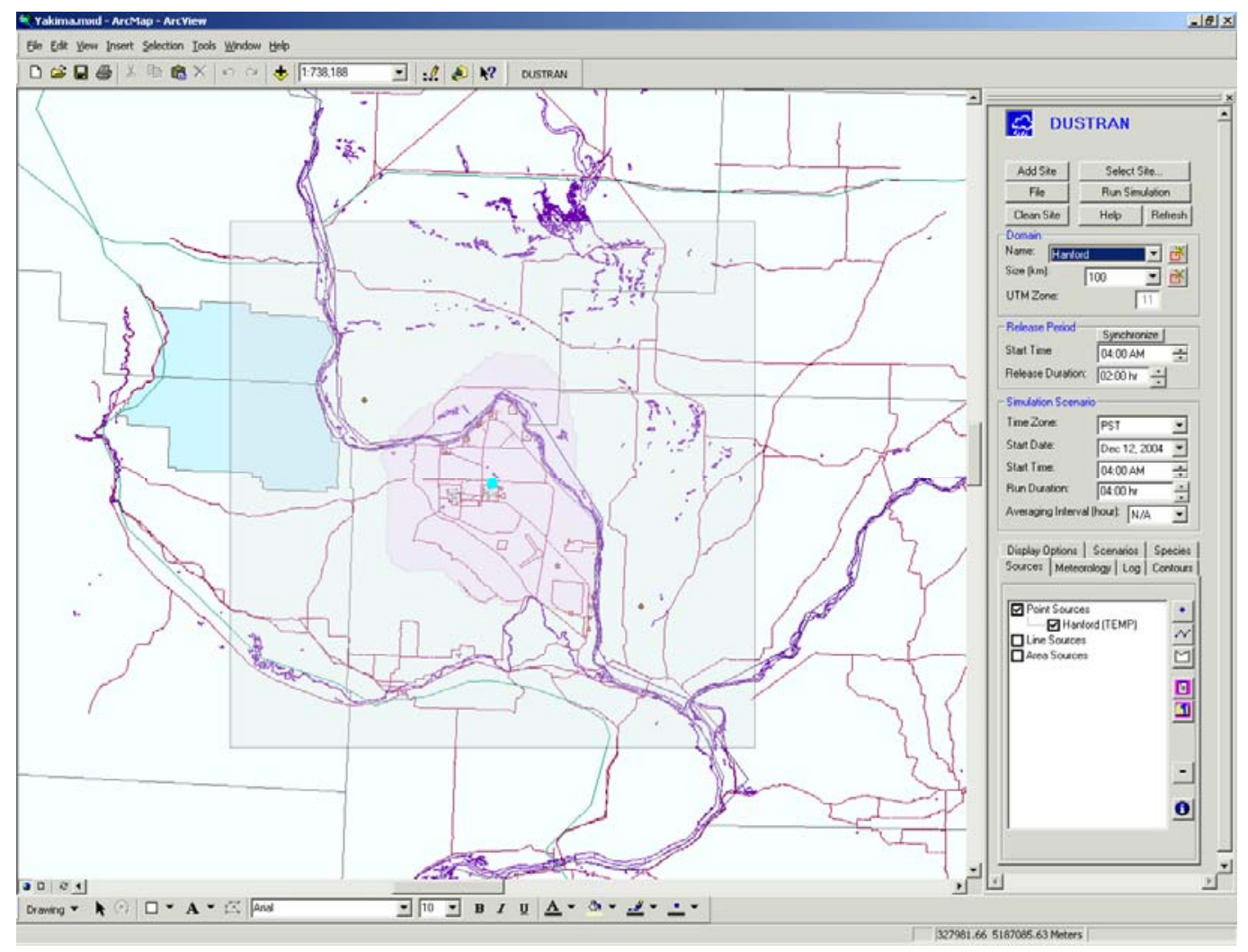

Figure 4.1. Site Loaded into the DUSTRAN Modeling System 


\subsection{Domain Panel}

The "Domain" panel within the DUSTRAN interface is used to set the location and size of the modeling domain that will be used in a model simulation. The modeling domain is the area where both the meteorological and dispersion calculations are performed.

The "Domain" panel allows for domains to be selected, added, and deleted. Figure 4.2 displays an example of the "Domain" panel with an existing domain name and size selected.

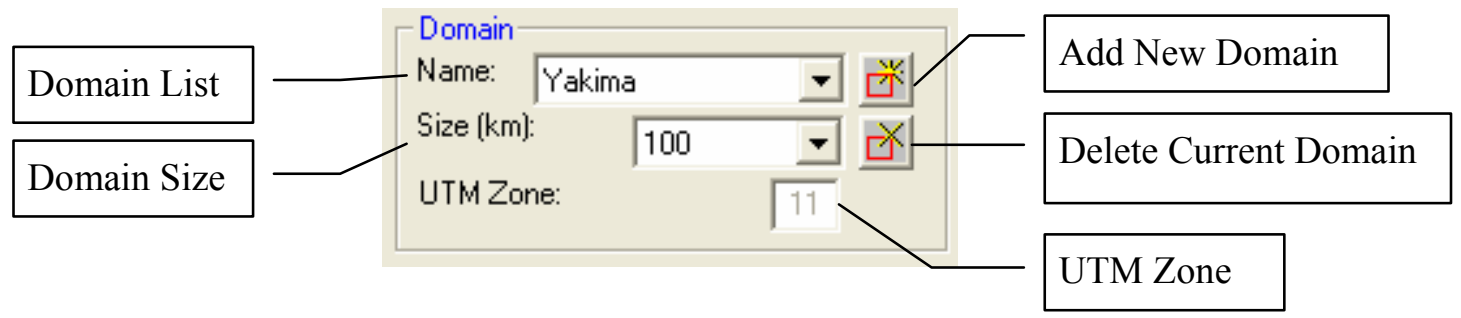

Figure 4.2. Example Domain Panel with Controls Labeled

\subsubsection{Creating a New Domain}

Multiple model domains can be created for a site, but only one model domain can be used in a given simulation. To create a model domain:

- Within the "Domain" panel, click on the “Add Domain”

- Select the location of the center of the new domain by clicking on a map location within ArcMap.

- When prompted, enter a name for the domain. This name will be added to the list of domains that are stored within the "Name" list; these domains are available for use in a given simulation.

- Set the domain size by selecting a size from the "Size" list. Domain sizes available for use within DUSTRAN include square areas that are 20, 50, 80, 100, 150, 200, 250, 300, 350, and 400 kilometers on a side.

- Following the selection of a domain size, ArcMap's map display will refresh automatically to the selected domain location, and the domain boundary will be sized appropriately.

\subsubsection{Selecting an Existing Domain}

Domains that currently exist for a site are available for selection under the "Name" list. To select an existing domain:

- Within the "Domain" panel, select a desired domain from the "Name" list.

- The domain will automatically display in ArcMap. To change the size of the domain, select a size from the "Size" list.

- Following the selection of a domain size, ArcMap's map display will refresh automatically to the selected domain location, and the domain boundary will be sized appropriately. 


\subsubsection{Deleting a Domain from a Site}

A previously created domain can be deleted from a site so that it no longer appears as an available domain under the "Name" list. To delete a domain:

- Within the "Domain" panel, select the name of the domain to delete from the "Name" list.

- Click on the "Delete Domain"

\subsection{Release Period Panel}

The "Release Period" panel is used to set a default start time and duration for all newly created sources. The start time and duration for a given source can be set independently of the default value at the time of the source's creation (see the Section 4.6 for information on creating sources). In addition, the "Release Period" panel can be used to synchronize each selected source's start and end times. Source synchronization is normally performed after all sources have been created and is a convenient method for assigning sources the same release time and duration, if desired. Figure 4.3 displays an example of the "Release Period" panel with the input controls labeled.

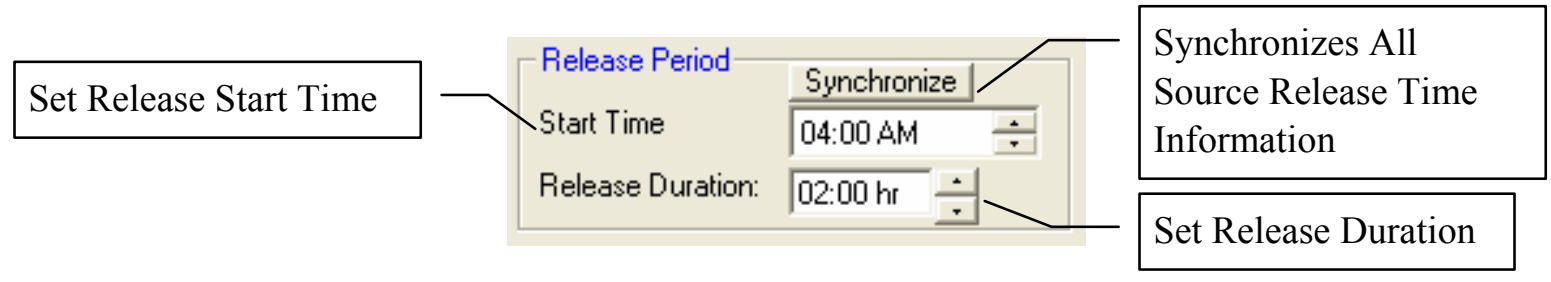

Figure 4.3. Example Release Period Panel with Controls Labeled

\subsubsection{Setting the Default Release Start Time and Duration}

A default "Start Time" and "Release Duration" can be set for sources on the "Release Period" panel. The "Start Time" is the default starting time in which sources begin releasing material. The "Release Duration" is the default period for which material is released. These values are used as defaults whenever a source is created; they can be changed specifically for each source on the "Release Parameters" form for that source.

To change the default "Start Time":

- Enter an hour value or click the increase or decrease arrow buttons for the "Start Time" input box. Clicking the up-arrow (down-arrow) will increase (decrease) the time by 1 hour.

- Currently in DUSTRAN, start times are limited to hour increments, and any minutes entered will be ignored by the simulation.

To change the default "Release Time":

- Enter an hour value or click the increase or decrease arrow buttons for the "Release Duration" input box. Clicking the up-arrow (down-arrow) will increase (decrease) the time by 15 -minute intervals. Currently, the model simulation uses only hour increments, and any minutes will be ignored. 


\subsubsection{Synchronizing the Release Start Time and Duration for all Sources}

All start times and durations can be "Synchronized" to the same time and period for all selected sources. However, synchronization should be used with caution to avoid inadvertently resetting release start times and durations to unintended values. Synchronization is typically performed after all sources have been created via the "Source" tab, but only if the user wants all source start times and release durations to be the same. To synchronize source times:

- Set the default "Start Time" and "Release Duration" on the "Release Period" panel.

- Click on the "Synchronize" button. A dialog box will be displayed asking the user to confirm changing the time data for all of the sources. Selecting the "OK" option will result in the time data for all sources to be changed to the default values found in the "Release Period" panel. Selecting the "Cancel" option will cancel the synchronize operation, and no changes will occur.

\subsection{Simulation Scenario Panel}

The "Simulation Scenario" panel is used to set the simulation type, start date, time, and run duration. The start date, time, and run duration correspond to the time that the CALMET and CALPUFF or CALGRID models are run and do not need to correspond with source release start times or durations. Often, the run duration is chosen so that it is longer than the release duration to continue simulating the plume movement and diffusion on the domain after all sources have finished releasing material. Figure 4.4 shows an example of the "Simulation Scenario" panel with sample entries.

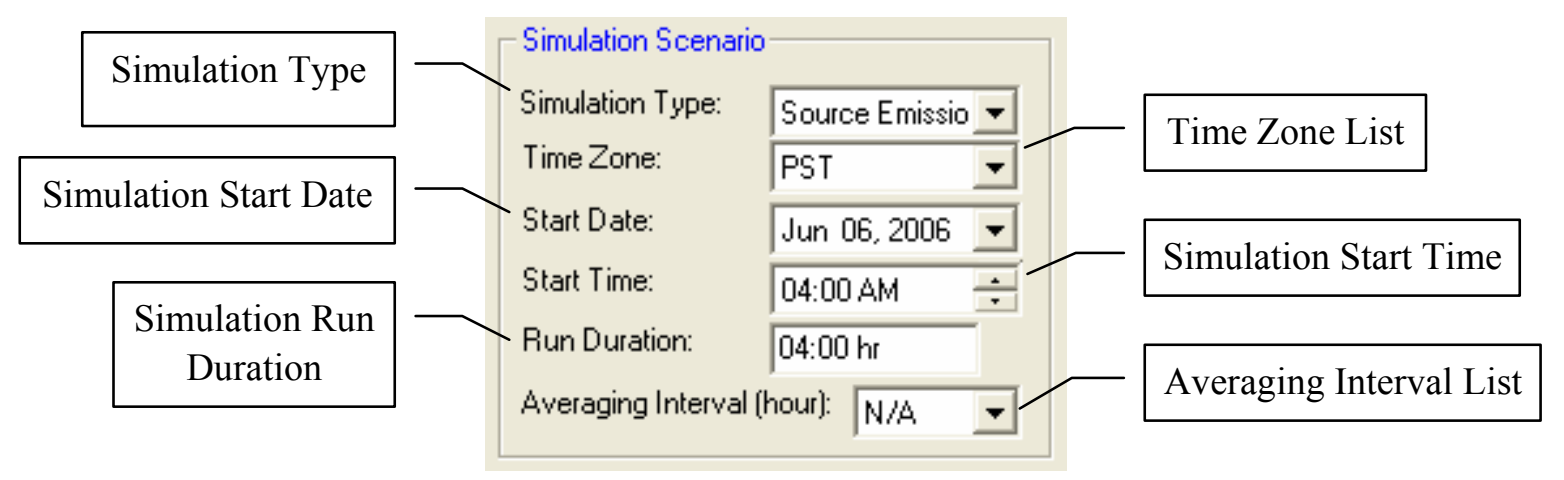

Figure 4.4. Example Simulation Scenario Panel with Controls Labeled

\subsubsection{Setting the Simulation Type}

The simulation type is used to distinguish between which dispersion model (CALPUFF or CALGRID) is used to run a given simulation. In DUSTRAN, CALPUFF is used to model standard "Source Emissions" and includes emissions that can be quantified using point, area, or line-source types. "Vehicular dust emissions," which can be quantified using area or line-source configurations, is an example source type that should be run using this mode. CALGRID is used to model "Wind-blown Dust" dispersion over the modeling domain. A qualitative overview of both models is provided in Sections 2.2 and 2.3 , respectively.

To set the dispersion model to use for the current "Simulation Type," select 
- "Source Emissions" to use the CALPUFF dispersion model for the current simulation. CALPUFF is ideal for modeling discrete sources that can be quantified using point, area, or linesource types. Emissions from stacks or roadways are example sources that would normally be run using this simulation type.

- "Wind-blown Dust" to use the CALGRID dispersion model for the current simulation. The wind-blown dust emissions provided to CALGRID are automatically calculated for each model grid cell within the modeling domain using the approach described in Section 2.5.2.

\subsubsection{Setting the Time Zone}

The time zone is set through the "Time Zone" listbox within the "Simulation Scenario" panel. Available time zones include Pacific Standard (PST), Central Standard (CST), Mountain Standard (MST), and Eastern Standard (EST). The time zone is used to correct upper-air soundings to local time and is also used by the CALMET meteorological model to calculate sunrise and sunset for sensible heat flux calculations.

\subsubsection{Setting the Start Date}

The start date for the simulation is entered in the "Start Date" textbox within the "Simulation Scenario" panel.

- To enter the date manually, click on the month, day, or year portion of the date displayed in the control. Once the appropriate portion of the date has been selected, the new value can be entered. All values should be entered as integers and are automatically checked for correctness. In the case of the month, it will convert the integer to the appropriate month name abbreviation.

- To enter the date using the calendar control, first click on the dropdown arrow. A calendar will be displayed for the date that is currently entered in the "Start Date" control. To change years, click on the year label within the calendar and click on the up (down) arrows to increment (decrement) by 1 year. To change months, click on the right or left arrow buttons located at the top of the calendar control. Clicking on the left (right) arrow buttons decreases (increases) the month by one. To select a day, click on the desired day within the calendar, and the calendar control will then close with the "Start Date" input box automatically updating to the new date.

\subsubsection{Setting the Start Time}

The start time for the simulation is entered in the "Start Time" textbox within the "Simulation Scenario" panel. The simulation start time can begin before any sources begin releasing material. In fact, the meteorological model, CALMET, requires the simulation to begin before sunrise, which for most of the year generally occurs after $5 \mathrm{a} . \mathrm{m}$. local time at most continental U.S. locations. This requirement exists because the CALMET-derived boundary layer parameters and mixing height are a function of the sensible heat flux, which in turn is function of sunrise. For most simulations, setting the "Start Time" to 04:00 a.m. adequately meets CALMET requirements.

To set the "Start Time":

- Click the "Start Time" textbox and enter the hour value manually or by clicking on the increase or decrease arrow buttons found to the right of the input box. Clicking the up-arrow (down- 
arrow) will increase (decrease) the time by 1 hour. Start times are currently limited to hour increments, and any minutes are ignored.

\subsubsection{Setting the Run Duration}

The run duration for the simulation is entered in the "Run Duration" textbox within the "Simulation Scenario" panel. The run duration is typically longer than any source release duration to allow for the CALPUFF or CALGRID dispersion models to continue calculating concentration and deposition values for any residual plume material in the modeling domain.

To set the "Run Duration":

- Click the "Run Duration" textbox and enter an hour value manually or by clicking on the increase or decrease arrow buttons found to the right of the input box. Clicking the up-arrow (downarrow) will increase (decrease) the time by 15 -minute intervals. Currently, the models simulate hour increments, and any minutes are ignored.

\subsubsection{Setting the Averaging Interval}

The "Averaging Interval" of a simulation is the number of hours to use when processing the average of the results generated by the CALPUFF or CALGRID dispersion models. The following "Averaging Intervals" are available:

N/A: No averaging will be performed for the simulation.

Note: the "N/A" interval corresponds to a model-default, 1-hour average.

3 Hours: 3-hour averaging will be used for the processing of results.

8 Hours: 8-hour averaging will be used for the processing of results.

24 Hours: 24-hour averaging will be used for the processing of results.

Run Length: Averages will be calculated using the entire length of the simulation run.

To set the "Averaging Interval":

- Select the desired interval from the "Averaging Interval" list found within the "Simulation Scenario" panel. If an averaging interval that is selected is greater than the "Run Duration," a message box will be displayed explaining the time mismatch.

\subsection{Species Tab}

The "Species" tab is used to view, add, and delete species from the current model scenario. Species can be either particles or gases, and their properties (diameter or molecular weight) can be set to allow for deposition calculations. Species that are "checked" are available for selection on all source forms when creating new or editing existing sources. Figure 4.5 shows an example of the "Species" tab with four species selected and the controls labeled. 


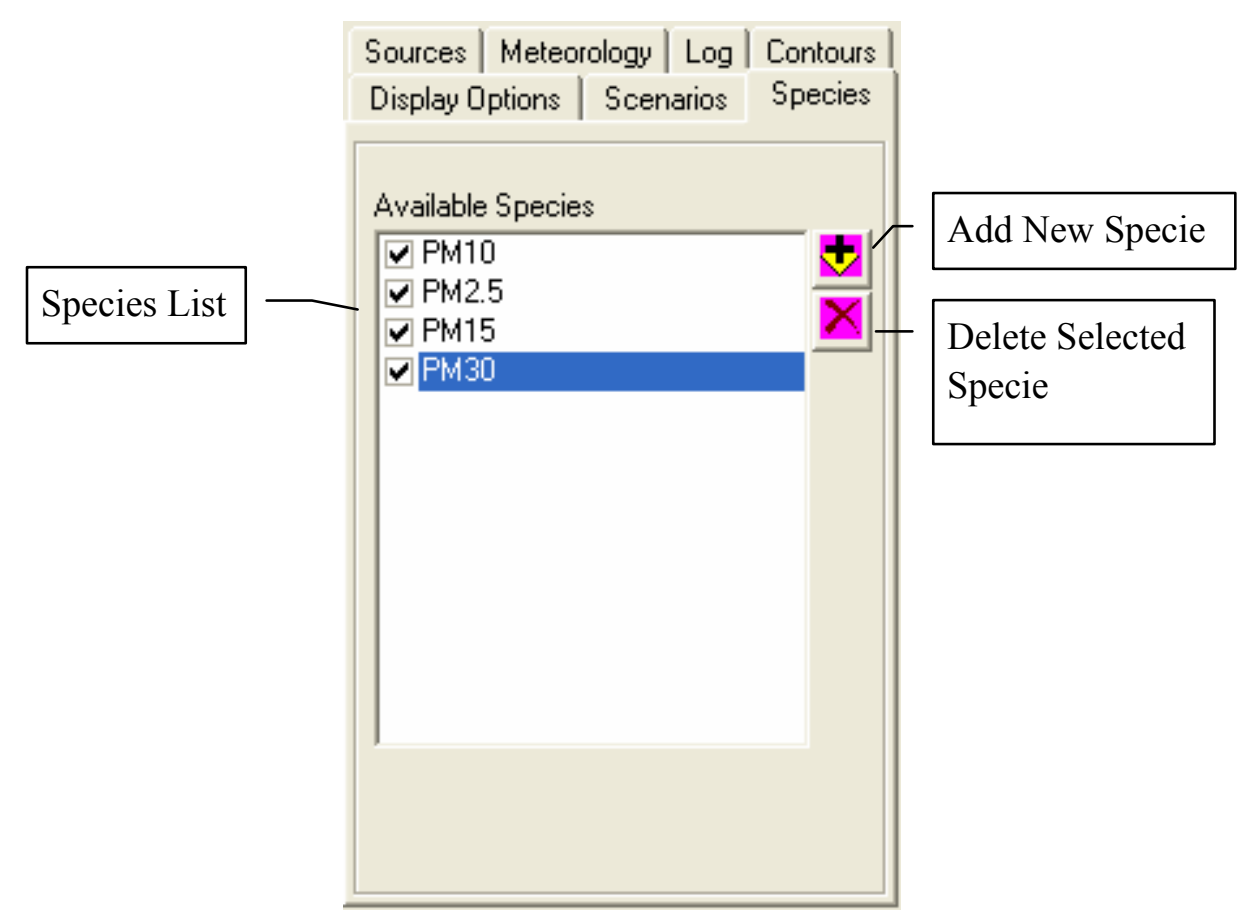

Figure 4.5. Example Species Tab

\subsubsection{Selecting an Existing Specie}

Existing species that are available for selection in a simulation are shown in the "Available Species" list on the "Species" tab. Species that are selected will appear on each source's "Release Parameter" form. To select an existing specie:

- Check the box located to the left of the specie. There are four default particulate matter (PM) species-PM10, PM2.5, PM15, and PM30. The number after the PM designation represents the mean particle diameter, in microns. Emissions for these four particle size categories are calculated automatically by the dust emission module within DUSTRAN. However, emission rates for user-specified species are not determined by DUSTRAN and must be entered directly for each source.

\subsubsection{Modify Data for an Existing Specie}

Existing specie data can be modified. For example, the mean diameter for a particle or the molecular weight for a gas can be changed. To modify an existing specie:

- Double-click on the name of the specie in the "Available Species" list on the "Species" tab. The "Specie Input Data" window will appear.

- To change the existing specie, click "Yes."

- Indicate whether the specie is a gas by clicking "Yes" or particle by clicking "No."

- If deposition calculations are desired, click "Yes"; otherwise click "No." For deposition, the program will prompt for the specie's mean diameter (particle) or molecular weight (gas). 


\subsubsection{Adding a New Specie}

New species, either particles or gases, can be added to a simulation. The specie will then be available on each source's "Release Parameter" form. To add a new specie:

- Click the "Add" $¥$ button located on the "Specie" tab. When prompted, enter a name for the new specie.

- Next, indicate whether the specie is a gas by clicking "Yes" or particle by clicking "No."

- If deposition calculations are desired, click "Yes"; otherwise click "No." For deposition, the program will prompt for the specie's mean diameter in $\mu \mathrm{m}$ (particle) or molecular weight (gas).

\subsubsection{Deleting an Existing Specie}

Existing species can be deleted from a simulation. Release information from existing sources for that specie will also be deleted. To delete a specie:

- Select the specie from the list and click the "Delete" $\$ button located on the "Species" tab.

\subsection{Sources Tab}

The "Sources" tab is used to add, edit, and delete sources within DUSTRAN. In addition, the sources tab is used to associate characteristic data files, such as vegetation and soil texture layers, which are used by the DUSTRAN modeling system to generate gridded dust emissions for a model domain when simulating "Wind-blown Dust." Various source types are available, including point, area, and line sources. Once a source has been created, it will appear on the source list within the "Sources" tab; each source appearing on the list can be edited and can be selected or deselected for use in the current simulation. Figure 4.6 shows an example "Sources" tab with the controls labeled and existing sources populated in the source list.

\subsubsection{Adding a New Point Source}

A point source, such as a stack emission, is modeled as a discrete point that can include the effects of plume rise due to buoyancy and momentum. A new point source can be added to a DUSTRAN simulation by doing the following:

- Click on the "Point Source" - button. A prompt will appear directing the user to select a location in the ArcMap map window for the point source. Click "OK."

- The mouse cursor will change to a crosshair; move the crosshair to the desired location for the point source on the map within ArcMap and click. Enter a name for the point source in the dialog box that appears and click "OK."

- The point source will appear in the source list under "Point Sources" and will also be displayed within ArcMap.

A "Source Input" window will appear with two tabs - "Release Parameters" and "Coordinates." "Release Parameters" are editable parameters that describe the release for the point source.

"Coordinates" are the UTM easting and northing coordinates for the source and are not editable. 


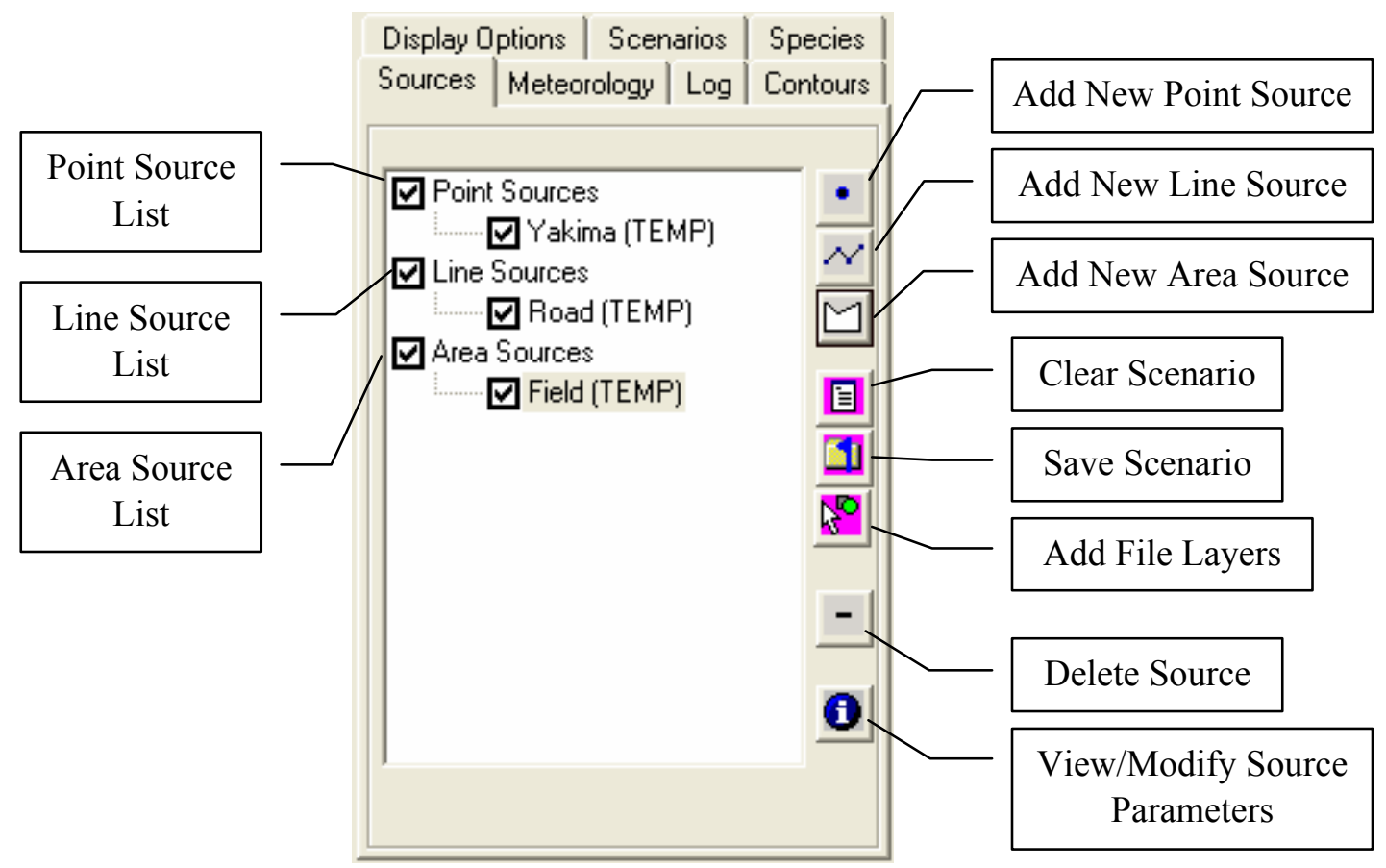

Figure 4.6. Example Sources Tab with Point, Line, and Area Sources Entered

\subsubsection{Setting Point-Source Release Parameters}

Once a new point source has been added or the name of an existing point source has been doubleclicked on the "Sources" tab, the point "Source Input" window will be displayed. The "Release Parameters" tab is selected by default and is used to enter the characteristics of the point source, such as the stack diameter, release time period, and the emission rates of species emitted by the source. Figure 4.7 displays an example "Release Parameters" form for a point source.

The following input parameters are available on the "Release Parameters" tab for a point source:

- Height of release

- Stack gas exit velocity

- Stack gas exit temperature

- Stack diameter

- Building cross section

- Initial horizontal plume size

- Initial vertical plume size.

Default values are provided for each parameter. To change a parameter's value, click on the textbox and enter a value. For quality assurance, textbox entries that are valid will appear green; invalid textbox entries will appear red. A range of valid entries appears in the yellow label at the bottom of the form for a given textbox entry. Each parameter's default values, units, and range validation can be changed in the "Cal.par" file (See Appendix A, Section A.2 for more information). 


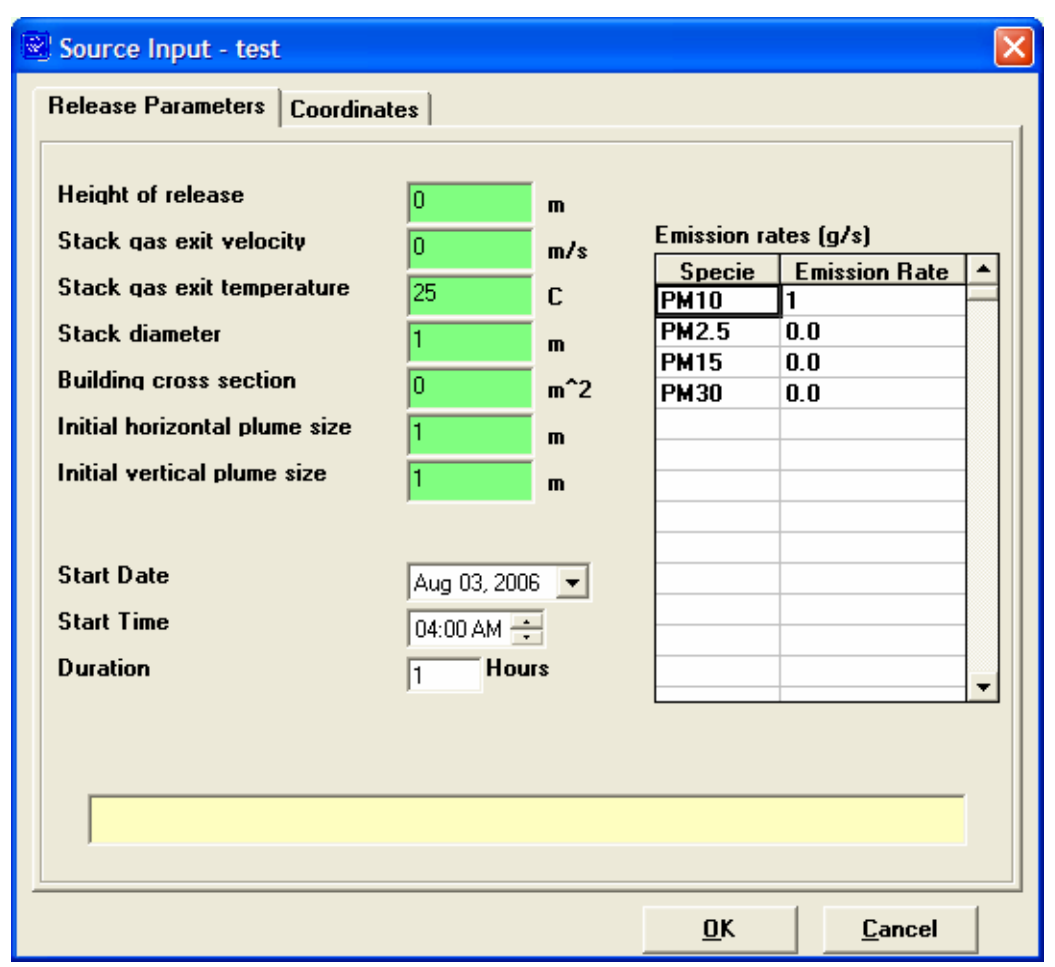

Figure 4.7. Example Point-Source Input Window

The point-source "Start Date," "Start Time," and "Duration" must also be entered on the "Release Parameter" tab. This is the date, time, and total period the source emits material in the simulation.

\section{$\underline{\text { Setting the Point-Source Start Date }}$}

- To enter the date manually, click on the month, day, or year portion of the date displayed in the control. Once the appropriate portion of the date has been selected, the new value can be entered. All values should be entered as integers and are automatically checked for correctness. In the case of the month, it will convert the integer to the appropriate month name abbreviation.

- To enter the date using the calendar control, first click on the dropdown arrow. A calendar will be displayed for the date that is currently entered in the "Start Date" control. To change years, click on the year label within the calendar and click on the up (down) arrows to increment (decrement) by 1 year. To change months, click on the right or left arrow buttons located at the top of the calendar control. Clicking on the left (right) arrow buttons decreases (increases) the month by one. To select a day, click on the desired day within the calendar, and the calendar control will then close with the "Start Date" input box automatically updating to the new date.

\section{Setting the Point-Source Start Time}

- Click the "Start Time" textbox and enter the hour value manually or by clicking on the increase or decrease arrow buttons found to the right of the input box. Clicking the up-arrow (downarrow) will increase (decrease) the time by 1 hour. Start times are currently limited to hour increments, and any minutes are ignored. 


\section{Setting the Point-Source Duration}

- Click the "Duration" textbox and enter an hour value manually or click on the increase or decrease arrow buttons found to the right of the input box. Clicking the up-arrow (down-arrow) will increase (decrease) the time by 15-minute intervals. Currently, the models simulate hour increments, and any minutes are ignored.

Emission rates for material being released from the point source are also entered on the "Release Parameters" tab. Emission rates can be set for several species (Note: Species are added in DUSTRAN through the "Species" tab; see Section 4.5.)

\section{$\underline{\text { Setting the Point-Source Emission Rates }}$}

- Click on the "Emission Rate" cell for a given "Specie" and enter its emission rate. If an "Emission Rate" is labeled "Not Selected," the specie must be activated on the "Species" tab in DUSTRAN. All emission rates are validated before closing the form.

\subsubsection{Adding a New Line Source}

A line source, such as emissions from roadways, is modeled as a series of line segments in DUSTRAN. Line-source emissions can be entered explicitly or calculated automatically when using the vehicular dust-emissions module for simulating dust emission from paved or unpaved roadways. A new line source can be added to a simulation by doing the following:

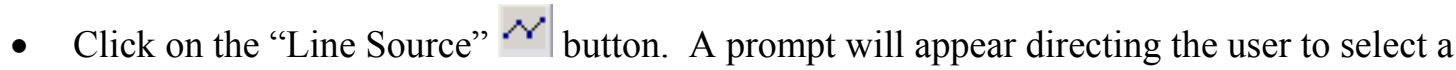
location in the ArcMap map window for the line source. Click "OK."

- The mouse cursor will change to a crosshair. The line source is drawn as a series of line segments. To create a line segment, click the mouse on the beginning and ending points of the segment. To complete the line source, double-click the mouse on the ending point of the last line segment. Enter a name for the line source in the dialog box that appears and click "OK."

- The line source will appear in the source list under "Line Sources" and will also be displayed within ArcMap.

A "Source Input" window will appear with two tabs - "Release Parameters" and "Coordinates." The "Release Parameters" tab contains editable parameters that describe the release for the line source. The "Coordinates" tab lists the UTM easting and northing coordinates for the source, and the coordinates are not editable. A third tab, called "Vehicle Parameters," is available if the "Emission Model" option is selected on the "Release Parameters" tab. The "Vehicle Parameters" tab is used to enter characteristic vehicle information for the line-source dust-emissions model.

\subsubsection{Setting Line-Source Release Parameters}

Once a new line source has been added or the name of an existing line source has been doubleclicked on the "Sources" tab, the line "Source Input" window will be displayed. By default, the "Release Parameters" tab is selected and is used to enter characteristics of the line source, such as the release height above ground level. Figure 4.8 displays an example "Release Parameters" form for a line source with the "Emission Model" option selected. 


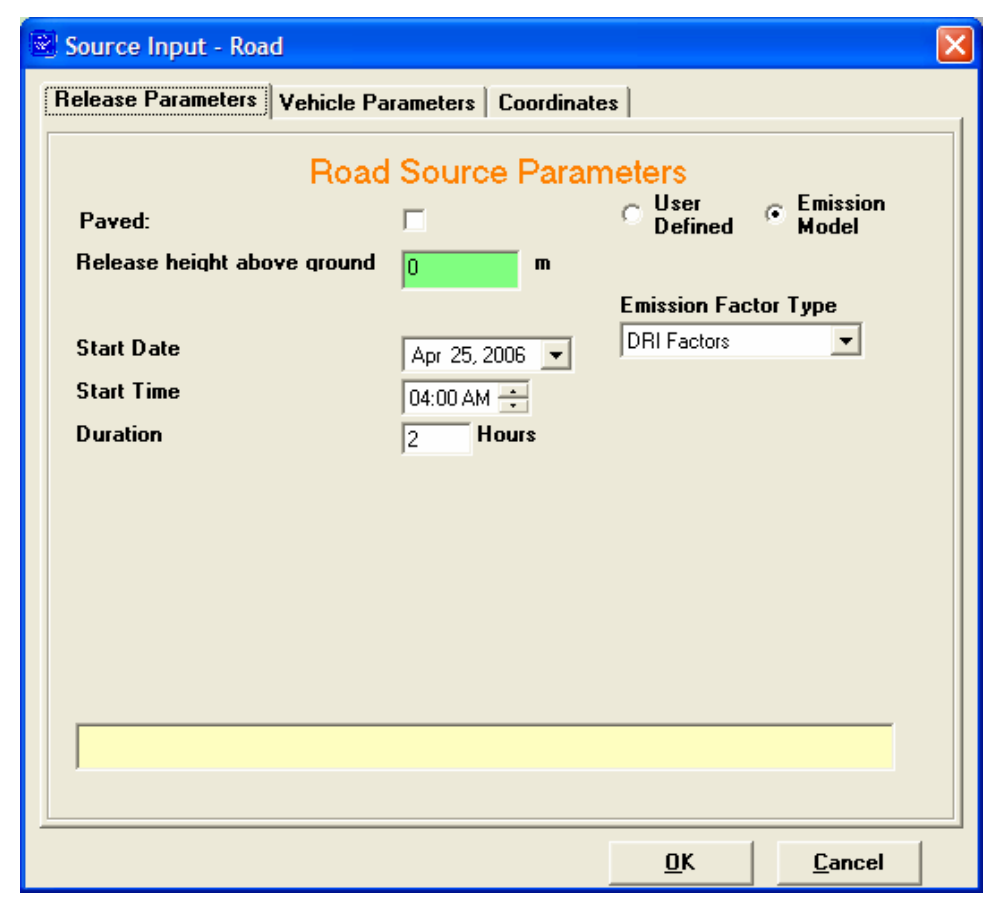

Figure 4.8. Example Line-Source Input Window Using the User Defined Emission Option

The "Release height above ground" setting is required for each line source and can include heights to accommodate elevated releases. For road emissions, the release height should be zero. To change the parameter's value, click on the textbox and enter a value.

The line-source "Start Date," "Start Time," and "Duration" must also be entered on the "Release Parameter" tab. This is the date, time, and total period the source emits material in the simulation.

\section{Setting the Line-Source Start Date}

- To enter the date manually, click on the month, day, or year portion of the date displayed in the control. Once the appropriate portion of the date has been selected, the new value can be entered. All values should be entered as integers and are automatically checked for correctness. In the case of the month, it will convert the integer to the appropriate month name abbreviation.

- To enter the date using the calendar control, first click on the dropdown arrow. A calendar will be displayed for the date that is currently entered in the "Start Date" control. To change years, click on the year label within the calendar and click on the up (down) arrows to increment (decrement) by 1 year. To change months, click on the right or left arrow buttons located at the top of the calendar control. Clicking on the left (right) arrow buttons decreases (increases) the month by one. To select a day, click on the desired day within the calendar, and the calendar control will then close with the "Start Date" input box automatically updating to the new date.

\section{$\underline{\text { Setting the Line-Source Start Time }}$}

- Click the "Start Time" textbox and enter the hour value manually or by clicking on the increase or decrease arrow buttons found to the right of the input box. Clicking the up-arrow (down- 
arrow) will increase (decrease) the time by 1 hour. Start times are currently limited to hour increments, and any minutes are ignored.

\section{Setting the Line-Source Duration}

- Click the "Duration" textbox and enter an hour value manually or click on the increase or decrease arrow buttons found to the right of the input box. Clicking the up-arrow (down-arrow) will increase (decrease) the time by 15-minute intervals. Currently, the models simulate hour increments, and any minutes are ignored.

Emissions for line sources can either be "User Defined" or calculated by the "Emissions Model" using EPA AP-42 (EPA 2005) or Desert Research Institute (Gillies et al. 2005a, 2005b) emission factors. Selecting "Emissions Model" causes the "Vehicles Parameters" tab to appear on the "Source Input" form (see "Setting Line Source Vehicle Parameters" for this option). If "User Defined" is selected, the emission rates can be entered directly on the "Release Parameters" tab. Emission rates can be set for several species (Note: species are added in DUSTRAN through the "Species" tab; see Section 4.5.)

\section{Setting the Line-Source Emission Rates}

- Click on the "Emission Rate" cell for a given "Specie" and enter its emission rate. If an "Emission Rate" is labeled "Not Selected," the specie must be activated on the "Species" tab in DUSTRAN. All emission rates are validated before closing the form.

\subsubsection{Setting Line-Source Vehicle Parameters}

The line-source "Vehicle Parameters" tab is activated on the line "Source Input" form by selecting "Emission Model" on the "Release Parameters" tab. The "Vehicle Parameters" tab is used to enter information about vehicles that will be traveling along the path of the line source. This information, which is used to calculate road dust emissions generated by vehicle traffic, includes the types of vehicles as well as their speed, weight, and the distance traveled. The particulate emission factors for wheeled vehicles operating on unpaved roads are empirically derived functions described in Section 2.5.1. Two calculation options are available: emissions using factors formulated by Desert Research Institute (Gillies et al. 2005a, 2005b), or emissions using factors calculated using EPA AP-42 recommendations (EPA 2005). Figure 4.9 shows the "Vehicle Parameters" form for a sample line source with vehicles selected.

A vehicle is used as an emission source by "checking" the box next to the vehicle's name. By clicking on a vehicle's name, a description of the vehicle is given in the "Vehicle Description" box and a set of input parameters for the vehicle is displayed. The vehicle-specific input parameters include:

- Number of Vehicles

- Vehicle Speed

- Vehicle Weight

- Road Length (non-editable - calculated by the interface).

As discussed in Section 2.2.5, DUSTRAN does not treat the motion of individual vehicles. Instead, a "bulk" approach is used to quantify dust emissions from vehicle activities. The dust emissions from all vehicles active on a road over a specified time are assumed to be released uniformly from the road at a constant rate throughout the duration of the activity. The input fields on the "Vehicle Parameters" form 
(Figure 4.9) do not represent the specific motions of individual vehicles; but rather an approach for providing the information needed by DUSTRAN the crate a "bulk" source term.

The approach for determining the input required on the "Vehicle Parameters" form (Figure 4.9) is to first estimate the total distance traveled along the road for all vehicles within a given vehicle type throughout the duration of the activity and then divide this total distance traveled by the road length to get the total effective "Number of Vehicles" on the form (Figure 4.9). Because the number of vehicles must be entered as a whole number, it is recommended that any fractional vehicles be rounded up to provide conservative estimates of dust emissions from the vehicle activities. The only constraint on the inputs on the form is that the product of the effective number of vehicles, vehicle speed, and activity duration be greater than the road length.

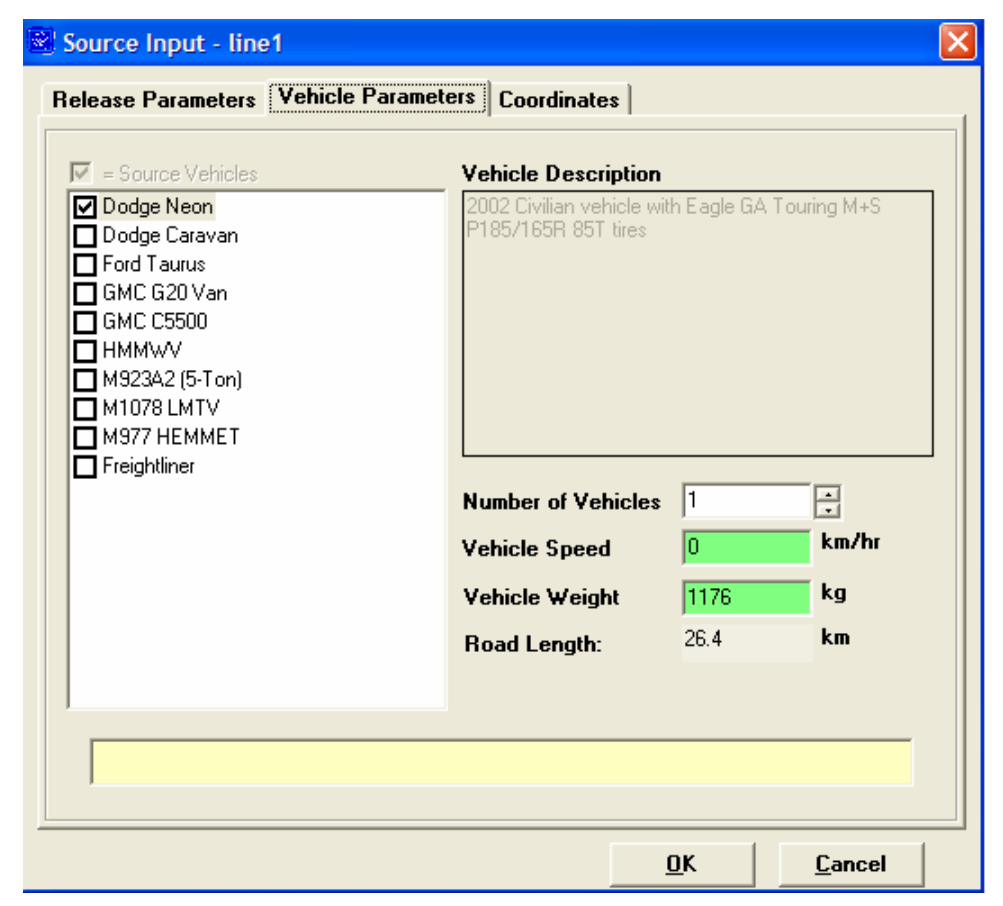

Figure 4.9. Example Vehicle Input Window for a Line Source

As described in Section 2.5.1, the dust emissions from a moving vehicle are proportional to the vehicle momentum (i.e., vehicle weight $\times$ vehicle speed). Therefore, if some vehicles of one type travel at significantly different speeds than other vehicles of the same type, another "vehicle type" will need to be added to the list such that the other speed(s) can be specified. Additional vehicles can be specified in DUSTRAN by editing the Cal.par file (see Section A.2.1).

For quality assurance, textbox entries that are valid (contained within an acceptable range) will appear green; invalid textbox entries will appear red. The range of acceptable values appears in the yellow label at the bottom of the form for a given textbox entry.

\subsubsection{Adding a New Area Source}

An area source, such as emissions from vehicles randomly crossing an off-road region, is modeled as a three- or four-sided polygon in DUSTRAN. Area-source emissions can be entered explicitly or 
calculated automatically when using the vehicular dust-emissions module for simulating dust emissions from paved or unpaved roadways. A new area source can be added to a simulation by doing the following:

- Click on the "Area Source" $\square$ button. A prompt will appear directing the user to select a location in the ArcMap map window for the area source. Click "OK."

- The mouse cursor will change to a crosshair. To create the area source, click the mouse at three or four points, depending on whether you want a triangle or a four-sided polygon. On the last point, double-click the mouse to complete the area source. Enter a name for the area source in the dialog box that appears and click "OK."

- The area source will appear in the source list under "Area Sources" and will also be displayed within ArcMap.

A "Source Input" window will appear with two tabs - "Release Parameters" and "Coordinates." The "Release Parameters" tab contains editable parameters that describe the release for the area source. The "Coordinates" tab lists the UTM easting and northing coordinates for the four corners of the area source and are not editable. A third tab, called "Vehicle Parameters," is available if the "Emissions Model" option is selected on the "Release Parameters" tab. The "Vehicle Parameters" tab is used to enter characteristic vehicle information for the area-source dust-emissions model.

\subsubsection{Setting Area-Source Release Parameters}

Once a new area source has been added or the name of an existing area source has been doubleclicked on the "Sources" tab, the area "Source Input" window will be displayed. By default, the "Release Parameters" tab is selected and is used to enter characteristics of the area source, such as the effective release height above ground level. Figure 4.10 displays an example "Release Parameters" form for an area source with the "User-Defined" emissions option selected.

The following input parameters are listed on the "Release Parameters" tab for an area source:

- Effective height above ground

- Air temperature

- Effective rise velocity

- Effective radius

- Initial vertical spread.

Default values are provided for each parameter. To change a parameter's value, click on the textbox and enter a value. For quality assurance, textbox entries that are valid will appear green; invalid textbox entries will appear red. A range of valid entries appears in the yellow label at the bottom of the form for a given textbox entry.

The area-source "Start Date," "Start Time," and "Duration" must also be entered on the "Release Parameter" tab. This is the date, time, and total period the source emits material in the simulation. 


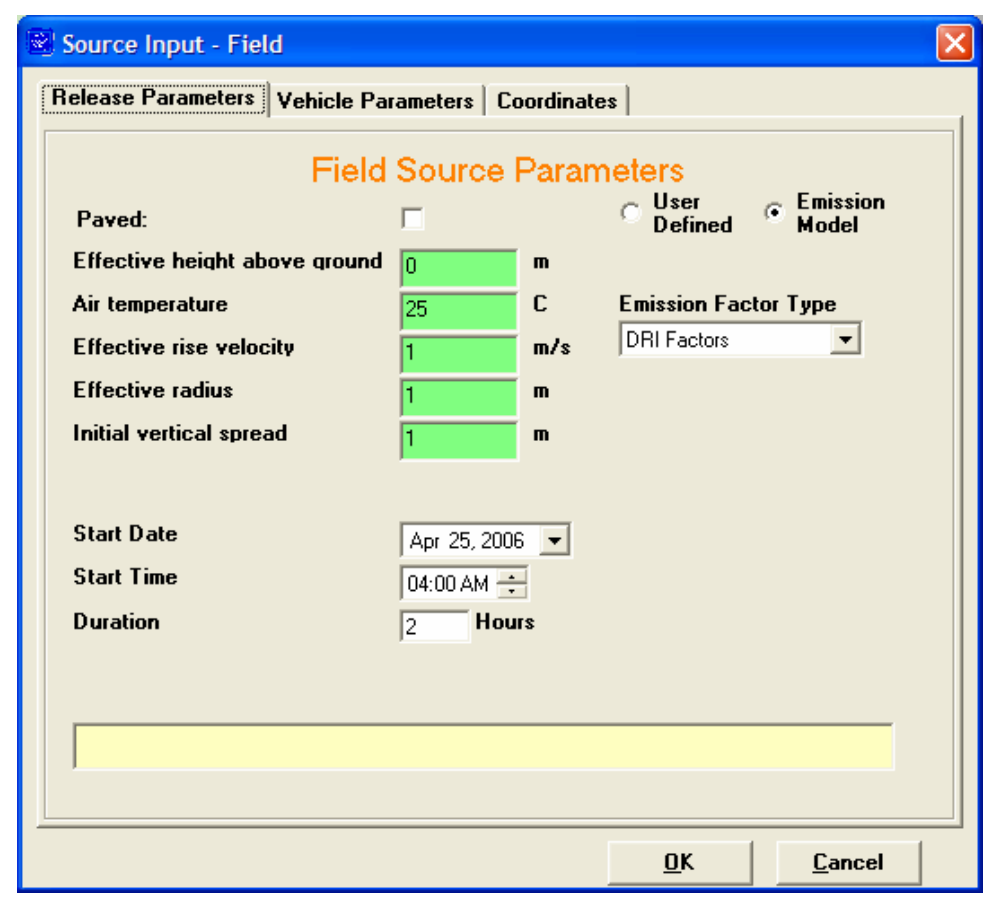

Figure 4.10. Example Area-Source Input Window Using the User Defined Emission Option

\section{$\underline{\text { Setting the Area-Source Start Date }}$}

- To enter the date manually, click on the month, day, or year portion of the date displayed in the control. Once the appropriate portion of the date has been selected, the new value can be entered. All values should be entered as integers and are automatically checked for correctness. In the case of the month, it will convert the integer to the appropriate month name abbreviation.

- To enter the date using the calendar control, first click on the dropdown arrow. A calendar will be displayed for the date that is currently entered in the "Start Date" control. To change years, click on the year label within the calendar and click on the up (down) arrows to increment (decrement) by 1 year. To change months, click on the right or left arrow buttons located at the top of the calendar control. Clicking on the left (right) arrow buttons decreases (increases) the month by one. To select a day, click on the desired day within the calendar, and the calendar control will then close with the "Start Date" input box automatically updating to the new date.

\section{$\underline{\text { Setting the Area-Source Start Time }}$}

- Click the "Start Time" textbox and enter the hour value manually or by clicking on the increase or decrease arrow buttons found to the right of the input box. Clicking the up-arrow (downarrow) will increase (decrease) the time by 1 hour. Start times are currently limited to hour increments, and any minutes are ignored.

\section{Setting the Area-Source Duration}

- Click the "Duration" textbox and enter an hour value manually or click on the increase or decrease arrow buttons found to the right of the input box. Clicking the up-arrow (down-arrow) 
will increase (decrease) the time by 15-minute intervals. Currently, the models simulate hour increments, and any minutes are ignored.

Emissions for area sources can either be "User Defined" or calculated by the "Emissions Model" using EPA AP-42 (EPA 2005) or Desert Research Institute (Gillies et al. 2005a, 2005b) emission factors. Selecting "Emissions Model" causes the "Vehicles Parameters" tab to appear on the "Source Input" form (see "Setting Area Source Vehicle Parameters" for this option). If "User Defined" is selected, the emission rates can be entered directly on the "Release Parameters" tab. Emission rates can be set for several species (Note: Species are added in DUSTRAN through the "Species" tab; see Section 4.5).

\section{Setting the Area-Source Emission Rates}

- Click on the "Emission Rate" cell for a given "Specie" and enter its emission rate. If an "Emission Rate" is labeled "Not Selected," the specie must be activated on the "Species" tab in DUSTRAN. All emission rates are validated before closing the form.

\subsubsection{Setting Area-Source Vehicle Parameters}

The area-source "Vehicle Parameters" tab is activated on the area "Source Input" form by selecting "Emission Model" on the "Release Parameters" tab. The "Vehicle Parameters" tab is used to enter information about vehicles that will be traveling within the area source. This information, which is used to calculate road dust emissions generated by the vehicles, includes the types of vehicles as well as their speed, weight, and the distance traveled. The particulate emission factors for wheeled vehicles operating on unpaved roads are empirically-derived functions described in Section 2.5.1. Two calculation options are available: emissions using factors formulated by Desert Research Institute (Gillies et al. 2005a, 2005b) or emissions using factors based on EPA's AP-42 recommendations (EPA 2005). Figure 4.11 shows the "Vehicle Parameters" form for a sample area source with vehicles selected.

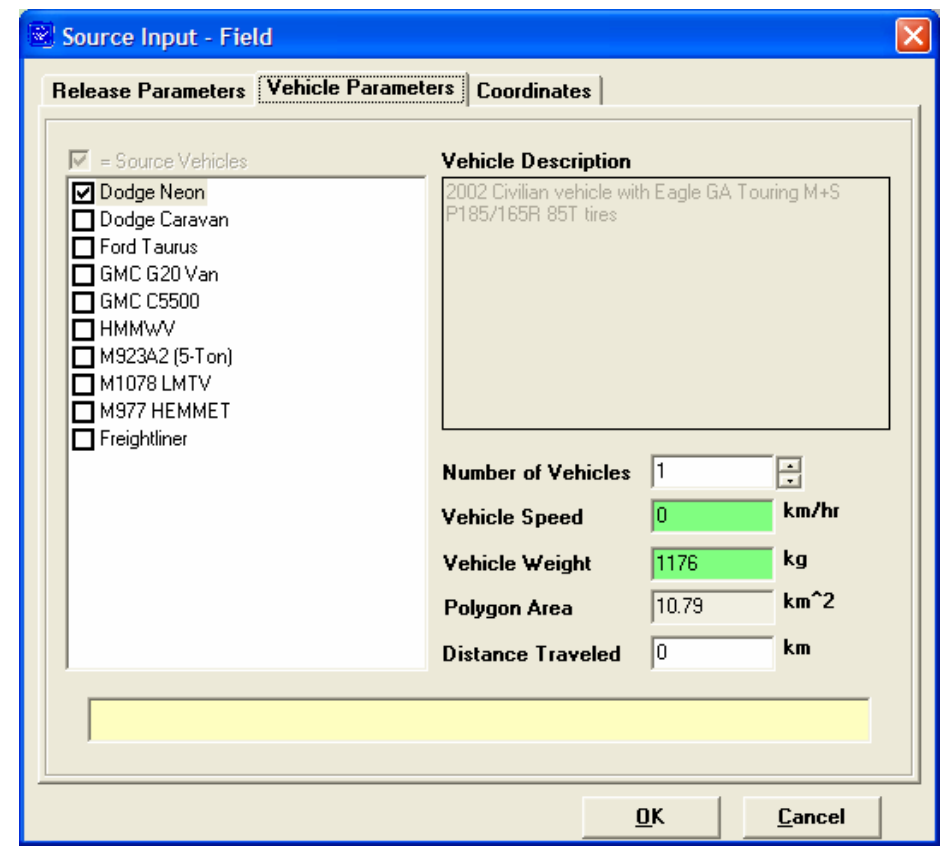

Figure 4.11. Example Vehicle Input Window for an Area Source 
A vehicle is defined as being an emission source by "checking" the box next to the vehicle's name. By clicking on a vehicle's name, a description of the vehicle is given in the "Vehicle Description" box, and a set for vehicle input parameters is displayed. The vehicle-specific input parameters include:

- Number of Vehicles

- Vehicle Speed

- Vehicle Weight

- Polygon Area (non-editable - calculated by the interface)

- Distance Traveled (by one vehicle during the duration of the release).

As discussed in Section 2.2.5, DUSTRAN does not treat the motion of individual vehicles. Instead, a "bulk" approach is used to quantify dust emissions from vehicle activities. That is, the dust emissions from all vehicles active in an area over a specified time are assumed to be released uniformly from the area at a constant rate throughout the duration of the activity. Therefore, the input fields on the "Vehicle Parameters" form (Figure 4.11) should not be interpreted as the specific motion of individual vehicles; the form is simply an approach for providing the information needed by DUSTRAN in a "bulk" sense.

The approach for determining the distance traveled by a vehicle within a specific vehicle type (specific weight and speed) is to estimate the total distance traveled for all vehicles within a vehicle type throughout the duration of the activity and then divide this total distance traveled by the total number of vehicles within a vehicle type to get the average distance traveled for one vehicle. This average value is the distance traveled specified in the input window (Figure 4.11). The only constraint on the inputs to the window is that the average distance traveled for one vehicle not be greater than the product of vehicle speed and activity duration. It is probable that the distance traveled will be less than the product of vehicle speed and activity duration because of the likelihood that not all vehicles of a particular type will be active during the entire period. For example, some vehicles may move intermittently (at their specified speed) during the period because of their particular function.

As described in Section 2.5.1, the dust emissions from a moving vehicle are proportional to the vehicle momentum (i.e., vehicle weight $\times$ vehicle speed). Therefore, if some vehicles of one type travel at significantly different speeds than other vehicles of the same type, another "vehicle type" will need to be added to the list such that the other speed(s) can be specified. Additional vehicles can be specified in DUSTRAN by editing the Cal.par file (see Section A.2.1).

For quality assurance, textbox entries that are valid will appear green; invalid textbox entries will appear red. A range of valid entries appears in the yellow label at the bottom of the form for a given textbox entry.

\subsubsection{Selecting Existing Sources to Use in a Simulation}

Multiple point, area, and line sources can be created for use in a simulation. After a source has been created, it will appear in a list on the "Sources" tab. To use the source in a simulation

- Check the "checkbox" next to the name of the source. The source will be displayed in the ArcMap map window and will be used in the model simulation.

- Check the checkbox next to that source type to select all sources for a particular source type (point, line, or area). 


\subsubsection{View and Edit Existing Source Information}

Existing point, line, and area sources appear in a source list on the "Sources" tab. To view and edit the input parameters for a particular source, double-click on the source's name or highlight the source

name and click on the "Information" $\mathbf{O}$ button. The "Source Input" form for that particular source will appear and is available for review and editing.

\subsubsection{Deleting an Existing Source}

Existing point, line, and area sources appear in the source list on the "Sources" tab. To permanently delete a source and its corresponding source information so that it is no longer available for use in a simulation, highlight the source's name and click on the "Delete" - button. The source will be removed from the simulation and will also be removed from display in the ArcMap map window.

\subsubsection{Clearing All Existing Sources}

Existing point, line, and area sources appear in the source list on the "Sources" tab. To permanently delete all sources and corresponding source information such that they are no longer available in the current simulation, click on the "New Scenario" 目 button. The sources will be removed from the simulation and will also be removed from display in the ArcMap map window.

\subsubsection{Saving Existing Sources as a Scenario}

Sources can be saved to a scenario that allows for the sources to be added to a simulation at a later time using the "Scenario" tab. A scenario is usually created after all sources and source parameters have been entered. To save the sources as a new scenario, click the "Save Scenario" 1 button. The scenario will also be added to the scenarios list, indicating that it is available for selection at a later time.

\subsubsection{Adding Characteristic Files (Soil and Vegetation for Wind-blown Dust)}

Soil and vegetation files that use the Zobler soil textures and Olson vegetation classes to characterize the underlying domain can be added to DUSTRAN for use in a "Windblown Dust" simulation. These files are created automatically at the time of the site's creation (see "Adding a New Site to DUSTRAN," Section 6.0) and must be associated with the scenario when performing a "Wind-blown Dust" simulation type. Alternatively, high-resolution soil and vegetation data files can be generated from ESRI shape files using the "Polygon Layer Creator Utility" discussed in Section 5.2. These detailed files can be used to more precisely define the underlying soil and vegetative surface characteristics for use in the wind-blown dust-emissions model.

To associate soil and vegetation characteristic files for use in a "Windblown Dust" simulation:

- Click the "Add Characteristic File" button. The "Characteristics Priorities" form, as shown in Figure 4.12, will appear. This form allows for files from the "Polygon Layer Creator" to be associated with the current scenario. Currently, two categories - Soils and Vegetation - are predefined and contain references to the standard Zobler soil texture and Olson Ecosystem class files created by the "Add Site" wizard within DUSTRAN.

- Click the "Add," "Higher," or "Lower" buttons for a given category to add a new characteristic file or to increase or decrease the file's usage priority. Generally, high-resolution files should be 
ranked higher in the list. A "checkbox" next to a file's name means the file will be used in the current simulation.

- Click "OK" to save the characteristic file selections or "Cancel" to ignore the most recent changes.

The "Polygon Layer Creator" button is used to launch the Layer Creator utility. This application allows for either manual or automatic (through ESRI shape files) creation of characteristic files for use in DUSTRAN (see Section 5.2, "Polygon Layer Creator Utility"). Although the existing soils and vegetation classification files are sufficient for running a "Wind-blown Dust" simulation in DUSTRAN, higher-resolution files can be associated with each category to provide better resolution. These files can be created within the "Polygon Layer Creator" tool, either manually or through user-specified shape files that use the Zobler or Olson classification schemes.

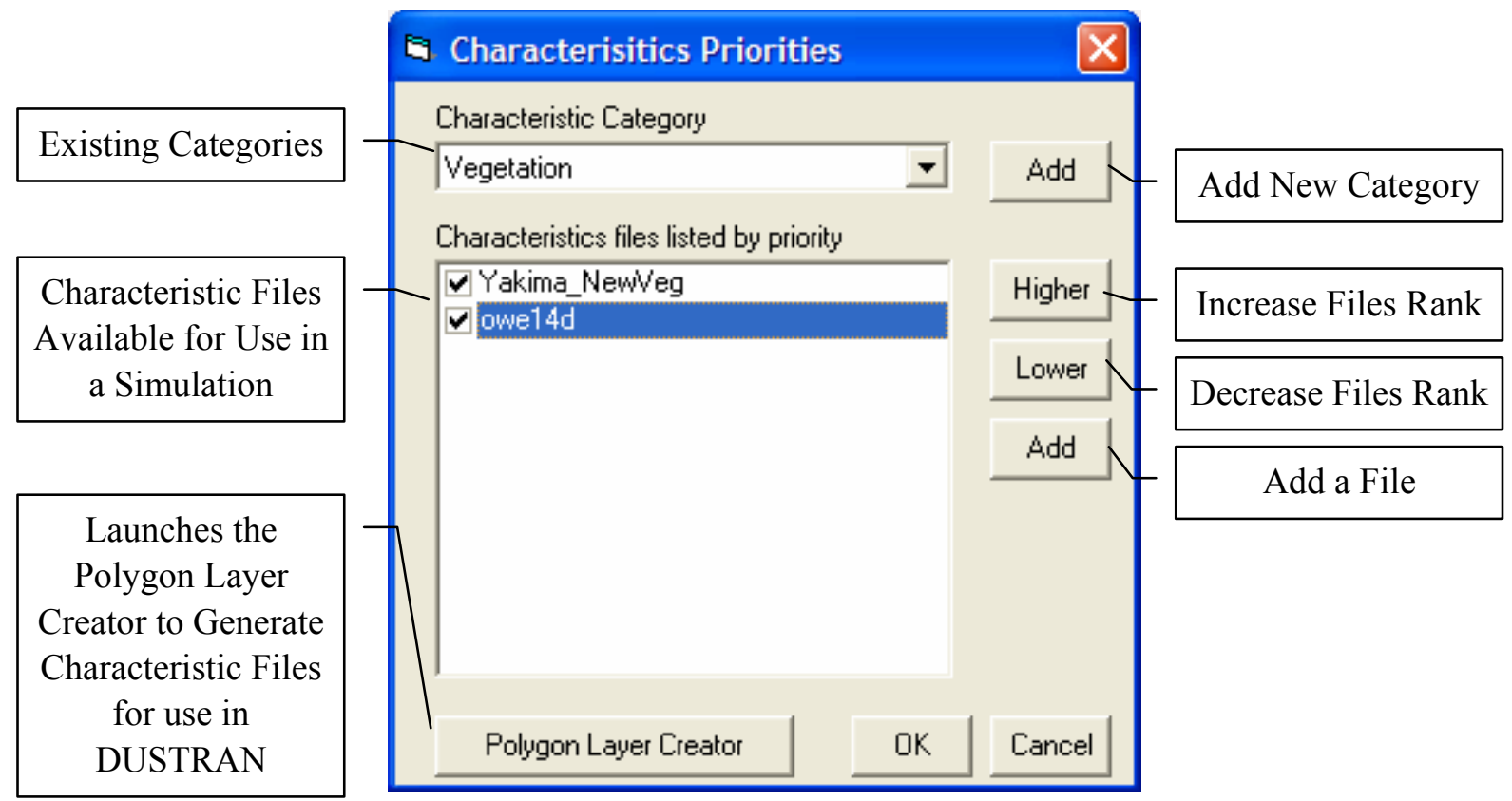

Figure 4.12. Characteristics Priorities Dialog Box for Adding Files, such as Soil and Vegetation Classes, in DUSTRAN

\subsection{Scenarios Tab}

The "Scenarios" tab is used to open or delete an existing scenario from the simulation. A scenario contains previously saved source information, including each source's location and parameter settings. A scenario gets created from the "Sources" tab (Note: To create a scenario, see Section 4.6, entitled "Sources Tab"). When selected, the "Scenarios" tab displays the current scenario name as well as a list of currently available scenarios for the site. Figure 4.13 shows the "Scenarios" tab with an example scenario entered. 


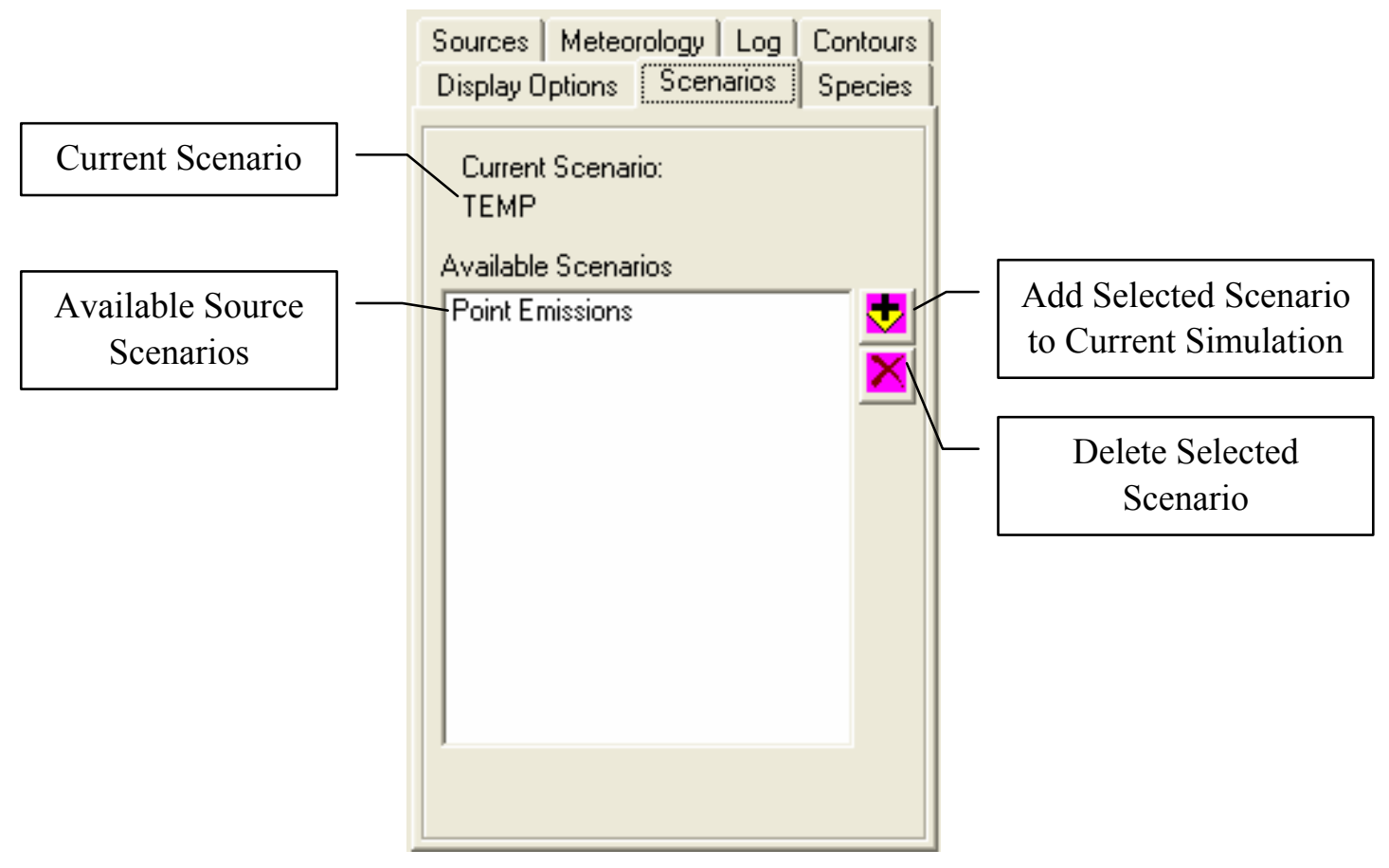

Figure 4.13. Example Scenario Tab with Controls Labeled

\subsubsection{Adding an Existing Scenario}

Previously saved sources (i.e., a scenario) can be opened for use in the current model simulation. To open a scenario:

- Click on the "Scenarios" tab. Highlight the scenario you wish to add by clicking on its name (Note: If no sources have been previously saved as a scenario, there will be no scenarios available in the list).

- Click the "Add Scenario" $¥$ button, and the scenario will be added to the simulation. All sources in the scenario will be listed on the "Sources" tab and displayed in ArcMap.

Multiple scenarios can be added to a simulation by following the steps above.

\subsubsection{Deleting an Existing Scenario}

Deleting a scenario permanently deletes all source and source-parameter information stored in that scenario from the site. Once the scenario is deleted from the site, it is no longer available for use in future simulations. To delete a scenario:

- Click on the "Scenarios" tab. Highlight the scenario you wish to delete by clicking on its name.

- Click the "Delete Scenario" $~ \$ button, and the scenario will be deleted from the list. 


\subsection{Meteorology Tab}

The "Meteorology" tab is used to select the surface and upper-air meteorological data sources for use in a simulation. Four options exist for specifying the meteorological data source in DUSTRAN:

- Available Data—for specifying site-specific (onsite) meteorological observation sources.

- Single Observation - for specifying meteorological conditions on a form within the DUSTRAN interface. These observations are for a single location (center of the domain) and persist for the duration of the simulation.

- User Defined - for specifying preformatted CALMET surface and upper-air observation files for direct use in DUSTRAN.

- NOAA (Archived) - for specifying archived NWS surface and upper-air data stations. (Note: To use this option, the meteorological data archive application-MetArchiver - must be running to populate the observations database. See Section 5.1 for more information).

Each of these options is discussed in more detail below and in Section 2.1.3.

\subsubsection{Selecting Available Data Meteorology}

Site-specific, onsite meteorological observation stations can be used in a DUSTRAN simulation by selecting "Available Data" from the "Use" list on the "Meteorology" tab. Because onsite data do not adhere to a universal format, DUSTRAN must be programmed to read the data. For example, currently two sites, Ft. Irwin and Yakima, have onsite data that have been integrated for use in the DUSTRAN interface.

To use onsite data in a DUSTRAN simulation:

- Select "Available Data" from the "Meteorology" tab.

- Check all "Surface Stations" and "Upper Air Stations" that are to be used in the simulation.

- Click the "Change Met Input Directory" to specify the directory where the surface and upper-air data files reside and then click "OK" to continue.

Figure 4.14 shows a sample "Available Data" window for the Yakima site, with a check next to each surface and upper-air station that will be used in the current DUSTRAN simulation.

\subsubsection{Selecting Single Observation Meteorology}

A single meteorological observation can be used for an entire simulation by selecting "Single Observation" from the "Use" list on the "Meteorology" tab. A form will appear, allowing for the entry of standard meteorological parameters that apply to a single point on the domain (i.e., the center of the domain). Even though the observation persists and is used for the entire simulation, the model-derived meteorological grids will still vary spatially and temporally, as they are a function of land use, topography, and the surface energy flux (i.e., time of day). 


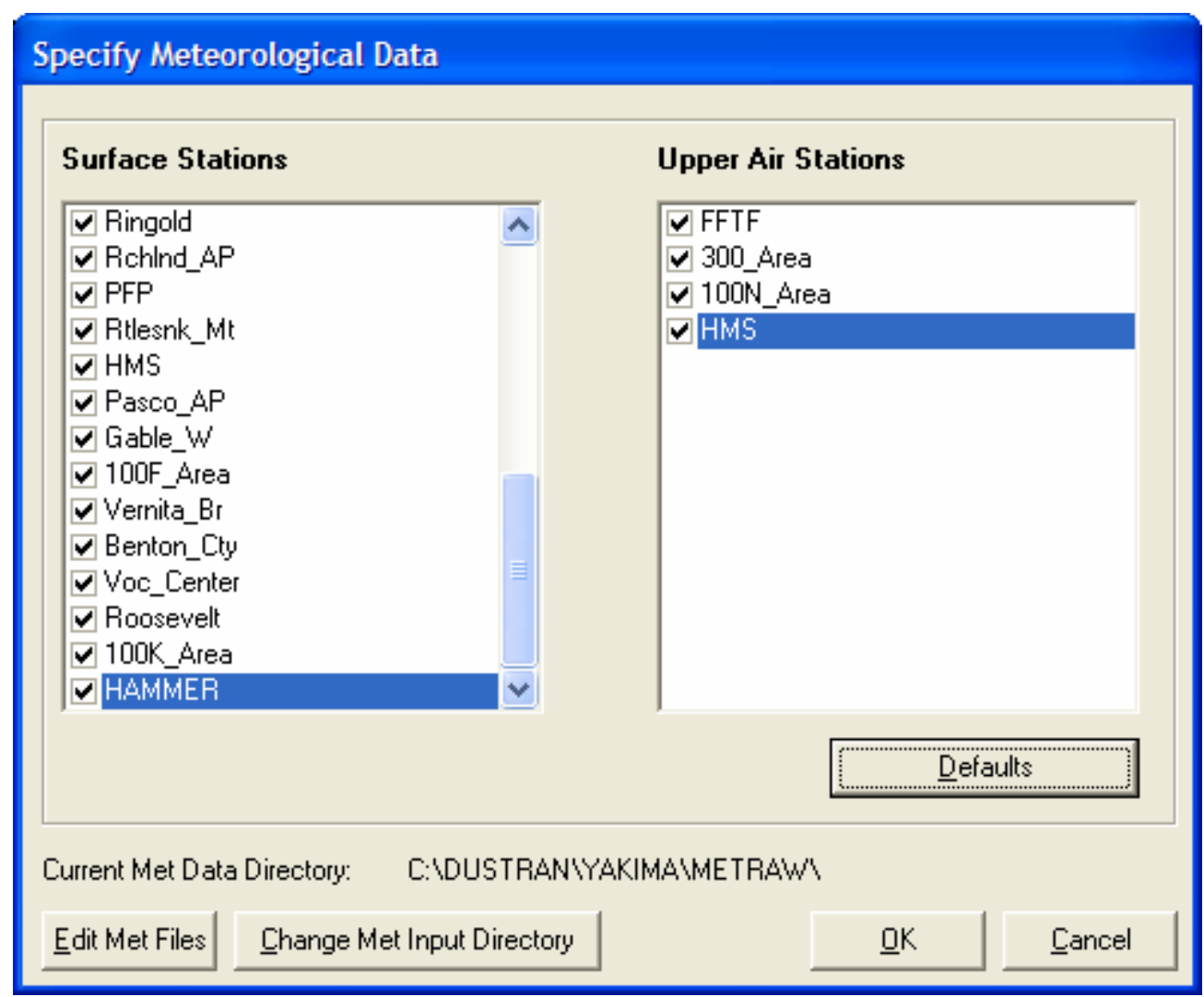

Figure 4.14. Onsite Data Selected Through the "Available Data" Option on the "Meteorology" Tab

To use a single observation in a DUSTRAN simulation:

- Select "Single Observation" from the "Meteorology" tab.

- Enter the meteorological observations on the "Specify Meteorological Data" form and then click "OK" to continue.

Figure 4.15 shows an example meteorology input window for the "Single Observation" option with default values entered for the parameters. Textboxes with valid entries are green; invalid characters or entries that are out of range will appear red. DUSTRAN uses the inputs on this form, along with the procedures and formulations detailed Section 2.1.3.1, to construct the necessary surface and upper-air profiles for use in CALMET. 


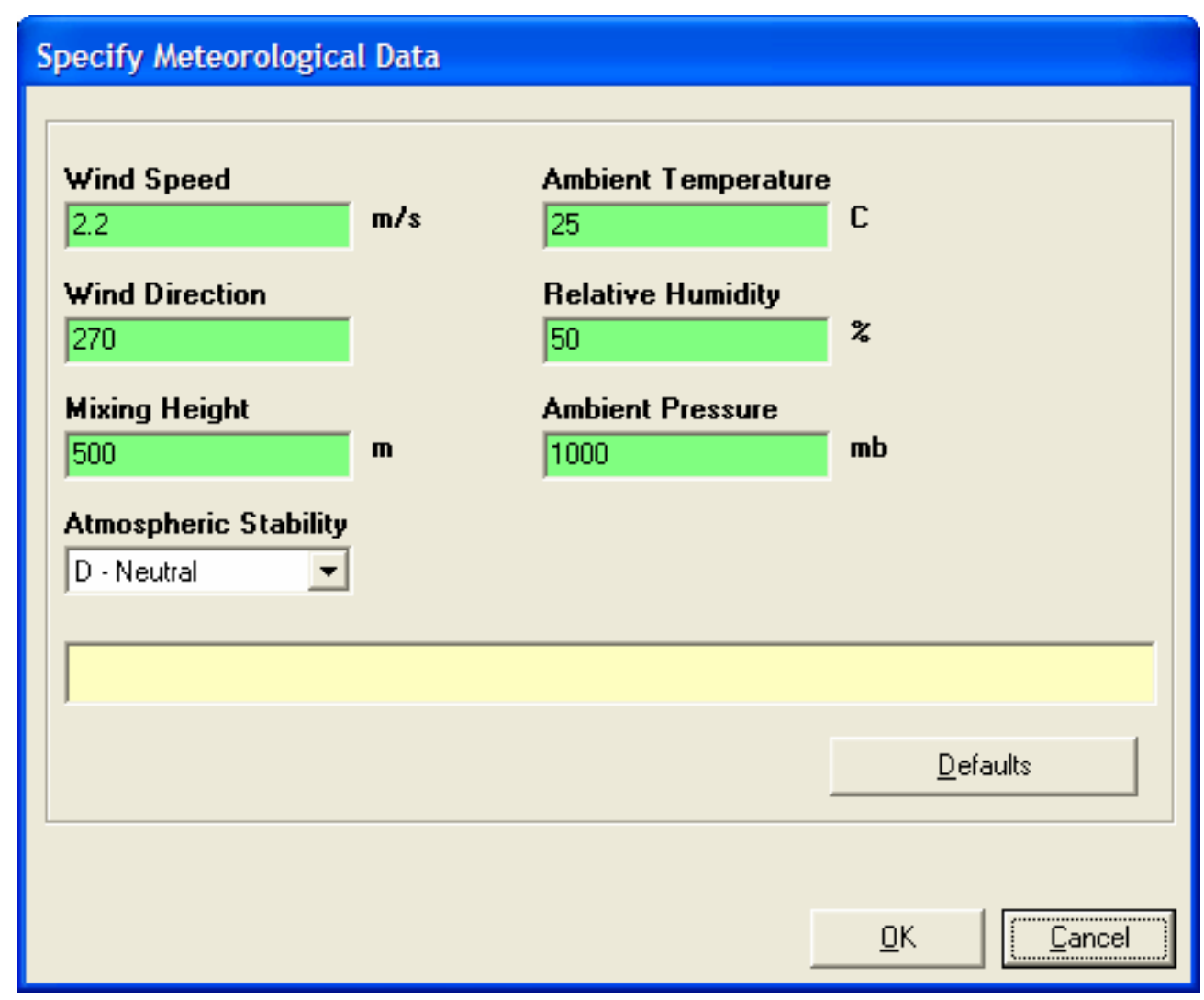

Figure 4.15. Single Observation Meteorology Input Window

\subsubsection{Selecting User Defined Meteorology}

User defined meteorological data can be used in a DUSTRAN simulation by selecting "User Defined" from the "Use" list on the "Meteorology" tab. This option requires the surface and upper-air meteorological data to already exist and to be in the correct format for CALMET. Specifically, the user must have created the CALMET surface (SURF.dat) and upper-air (UP.dat) files using a processing utility exterior to the DUSTRAN interface. For more information on the format of the meteorological input files and processing utilities for CALMET, refer to "A User's Guide for the CALMET Meteorological Model” (Scire et al. 2000b).

To associate existing CALMET-ready meteorological data files with DUSTRAN, two files must be created - a .snf (surface) and a .unf (upper-air) file. These are DUSTRAN-specific files and contain station metadata, such as station name, ID, and coordinate location. To create a .snf and unf to associate with existing CALMET SURF.dat and UP.dat files:

- Select "User Defined" from the "Meteorology" tab.

- Click the "Change Met Input Directory" to specify the directory where the CALMET-ready SURF.dat and UP.dat data files reside.

- Click on the "Edit Met Files" button. Select the "Surface Stations" tab and enter each station's Name, ID, Longitude, Latitude, Elevation, and UTM Easting and Northing coordinates. Note that the station ID must agree with the station ID(s) specified in the CALMET-ready SURF.dat file. 
- Select the "Upper Air Stations" tab and enter each station's Name, ID, Longitude, Latitude, Elevation, and UTM Easting and Northing coordinates. Note that the station ID must agree with the station ID(s) specified in the CALMET-ready UP.dat file.

- Click "OK" on the "Edit Met Station Data" form.

Figure 4.16 shows a sample "Surface Stations" tab that is used to specify the station information that is associated with the CALMET-ready SURF.dat file. The "Upper Air Stations" tab (not shown) is a similar form for entering upper-air station information that is associated with the CALMET-ready UP.dat files.

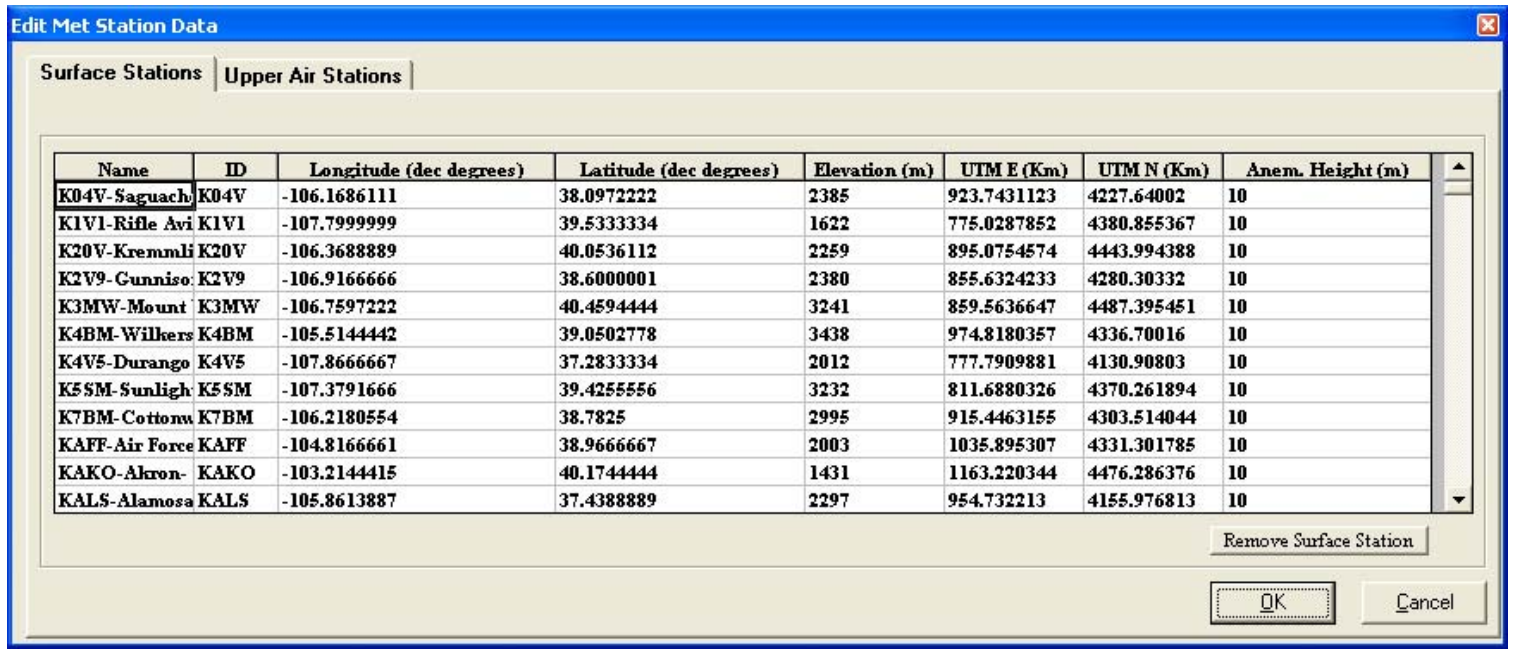

Figure 4.16. Sample "Surface Stations" Tab for Specifying CALMET-Ready Station Locations in DUSTRAN

\subsubsection{Selecting NOAA (Archived) Meteorology}

Archived NOAA meteorological data can be used in a DUSTRAN simulation by selecting "NOAA (Archived)" from the "Use" list on the "Meteorology" tab. Generally, all National Weather Service (NWS) hourly surface and twice-daily upper-air stations are available for direct use in DUSTRAN. These data are archived locally to an Access database using a separate utility, called "MetArchiver." MetArchiver is available in the root DUSTRAN directory and requires a continuous network connection to keep the archive database current. See Section 5.1 for more information on using "MetArchiver."

To use "NOAA (Archived)" data in a DUSTRAN simulation

- Select "NOAA (Archived)" from the "Meteorology" tab.

- In the "Open" file dialog box, select the Access database that is being used to archive meteorological data (Note: The database is created by the "MetArchiver" utility described in Section 5.1).

- Stations that are available for use in the simulation will populate the "Specify Meteorological Data" form. Click the checkbox next to the station's name to include the station in the analysis and click "OK" to continue. 


\subsection{Contours Tab}

The "Contours" tab is used to select the contours of interest to view for a given emission type and time period after completing a simulation in CALPUFF or CALGRID. Various contour types can be displayed, including concentration, exposure (i.e., time-integrated concentration), deposition, and total deposition for the simulated averaging period. Contours can be animated over the simulated period to provide a dynamic perspective of the plume advection and diffusion pattern. Figure 4.17 displays an example of the "Contours" tab following a simulation run.

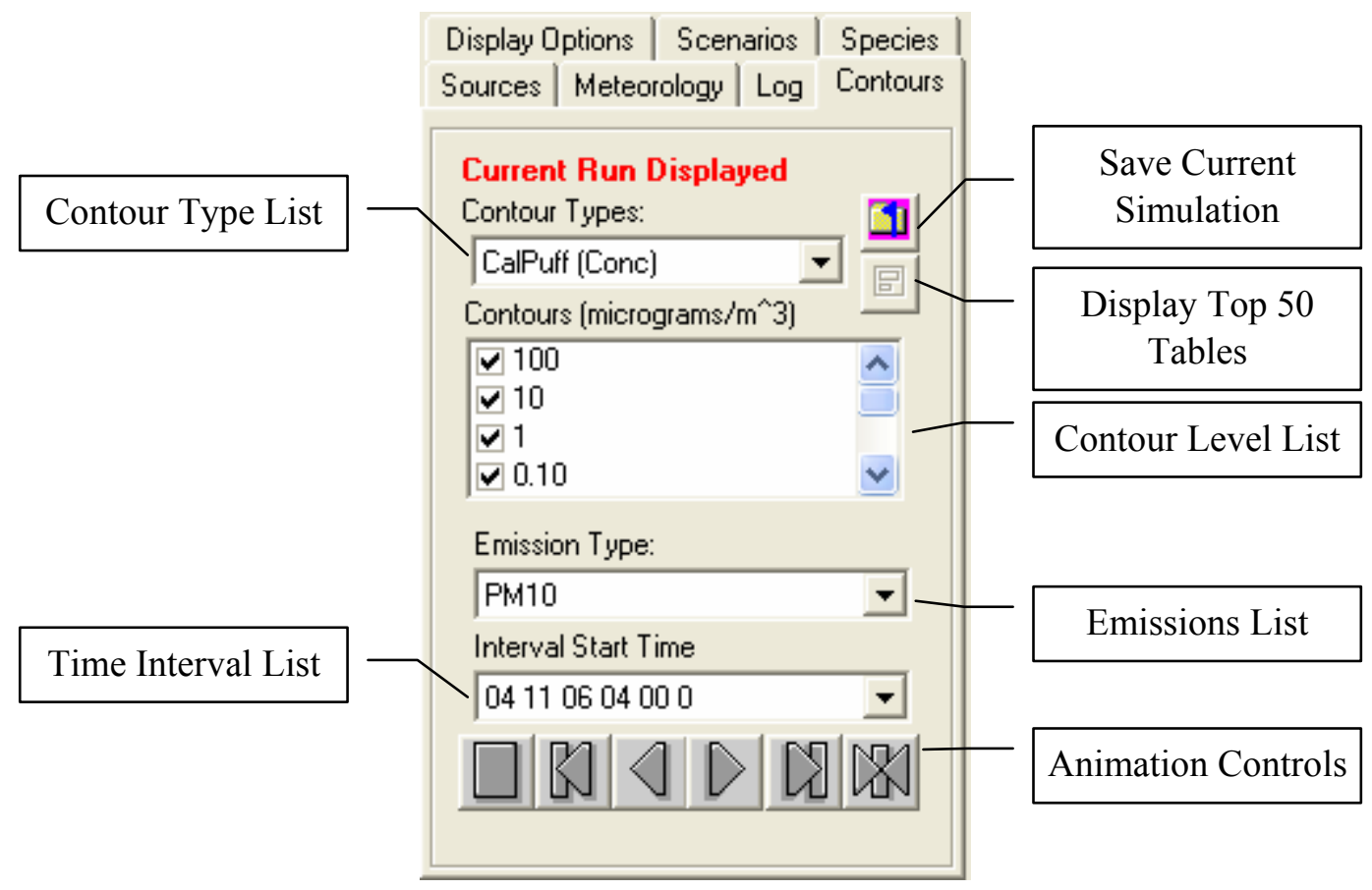

Figure 4.17. Example Contours Tab with Controls Labeled

\subsubsection{Setting the Contour Type}

After completing a simulation, various contour types are available for display in the ArcMap map window. To display the contour type of interest, select the "Contours" tab and select the desired "Contour Type" from the listbox. The dispersion model that generated the contours precedes the contour type name. Contour types include:

- "Conc"- the average air concentration. If "Conc" is followed by "AVG" and an integer value, then the concentration is for the averaging period defined by the integer.

- "Dep"- the average deposition. If "Dep" is followed by "AVG" and an integer value, then the deposition is for the averaging period defined by the integer.

- "Exp"- the total exposure (i.e., time-integrated concentration).

- "TotalDep"- the total, accumulated deposition. 


\subsubsection{Selecting Contour Levels}

After completing a simulation, various contour levels are available for display. Generally, these levels span many orders of magnitude to allow the user to clearly define the concentration or deposition footprint for a given scenario. To view the contour levels, select the "Contours" tab and check the desired levels within the "Contours" list to be displayed in the ArcMap map window. Uncheck any levels to remove them from display in the ArcMap map window. Typically, a plume ground-level footprint can reasonably be described over a range of 3-to-5 orders of magnitude. Displaying more than 5 orders of magnitude is primarily used to evaluate the model computations rather than having any semblance to a real plume. The number of contour levels and values of contour levels computed by DUSTRAN are specified in the Cal.par file described in Section A.2. Currently, DUSTRAN is set up for computing a concentration range spreading 12 orders of magnitude, which reasonably brackets expected concentrations over a range of emission and dispersion rates.

\subsubsection{Selecting Emission Type}

After completing a simulation, contours for a given emission type are available for display. The available emission type(s) correspond to the specie(s) selected on the "Species" tab before the model simulation. To set the emission type to display, click on the "Contours" tab and select a desired species from the "Emission Type" listbox. The contours for that particular emission type will be displayed in the ArcMap map window.

\subsubsection{Selecting Interval Start Time}

After completing a simulation, concentration and deposition contours can be displayed for any hourly time interval in the simulated period. To view the results for a particular time interval, select the "Contours" tab and choose the desired time interval from the "Interval Start Time" listbox. The listed times are in the following format: $\mathrm{mm}$ dd yy hh $\mathrm{min} \mathrm{sec}$, where $\mathrm{mm}$ is month, dd is day, yy is year, min is minutes, and sec is seconds. Averaged hourly results are referenced to the beginning of the "Interval Start Time." For example, to view the 0900 to 1000 hourly average concentration, select 0900 from the interval listbox.

\subsubsection{Animating Contours}

After completing a simulation, the displayed contours can be animated within the ArcMap map window to provide a dynamic perspective of the concentration or deposition pattern in time. The "Contours" tab contains a series of buttons to control the animation for the simulation. Figure 4.18 displays the animation control with each button labeled. The buttons (from left to right, in Figure 4.18) perform the following functions:

- $\quad$ Stop $\square$ : Stops the current animation at a given interval time step.

- Reset [D: Resets the contour animation to the first time step in the simulation.

- Back : Steps the contour animation back one time interval.

- Forward $D$ : Advances the contour animation one time interval.

- Run Once D: Runs contour animation once, sequentially displaying each interval in the simulation once. 
- Loop 俕: Continuously loops the contour animation.

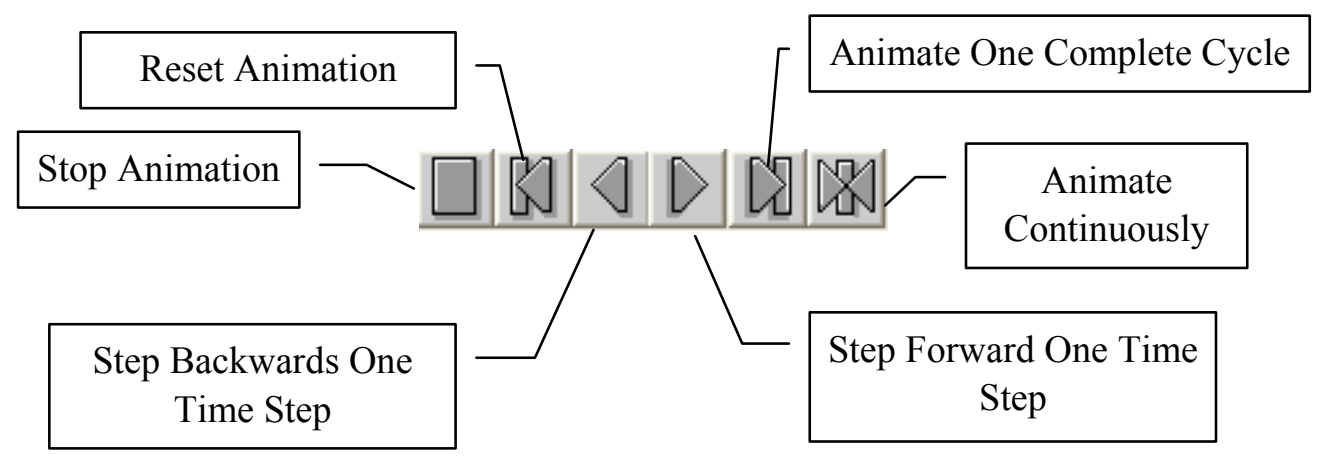

Figure 4.18. Animation Control with Buttons Labeled

\subsubsection{Saving Simulation Results}

After completing a simulation, the results can be saved for viewing at a later time. To save all results associated with a run, click on the "Contours" tab and click the "Save Current Run" 1 button. Enter a run name and description of the simulation in the prompts that follow. The run name is used to identify and open the simulation at a later time. The meteorological data are the only data that are not currently associated with a saved run. Consequently, the meteorological data must be associated with a saved run (through the "Meteorology" tab) to replicate previous simulations. When a simulation is saved, a text box shows that the analyst may be allowed to save comments with the simulation. Documenting the meteorological files associated with the saved simulation through the comment box will at least provide the necessary information to completely reconstruct previous simulations.

\subsubsection{Loading Results of a Saved Simulation}

A prior run that has been saved can be loaded into the modeling system by first clicking on the "File" button found on the main DUSTRAN interface. Once the "File" button has been clicked, the "Prior Run Selection" window will be displayed with a list of saved simulation runs. Click on the name of the saved simulation to open and click "OK" to load results. All data concerning the saved run (other than the meteorological data, as mentioned above) will be automatically loaded into the DUSTRAN interface, including the result contours. Figure 4.19 displays the Prior Run Selection window with an example run selected. 


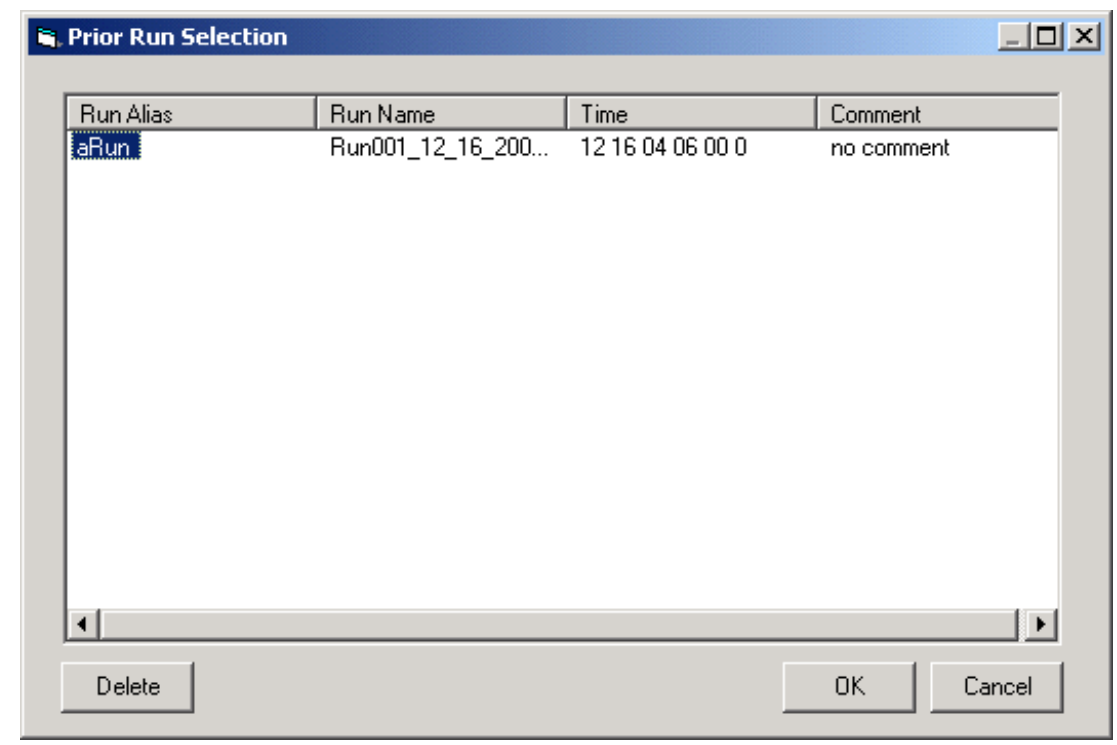

Figure 4.19. Example Prior Run Selection Window

\subsection{Display Options Tab}

The "Display Options" tab allows for controlling the rendering of certain items within the ArcMap map window, including contours, receptors, wind vectors, surface meteorological stations, and upper-air station locations. In addition, the appearance, such as the symbol size, shape, or color, for some of the items can be adjusted. This tab is usually visited after making a DUSTRAN simulation to control which objects to display in the ArcMap map window. Figure 4.20 is an example of the "Display Options" tab showing the available items for displaying in ArcMap after completing a simulation:

\subsubsection{Displaying the Contour Results}

The contours generated by the simulation can be viewed in the ArcMap map window by clicking on the checkbox labeled "Contours" on the "Display Options" tab. In addition, the color, size, and other properties of the displayed contours can be adjusted by clicking on the colored box next to the checkbox. See Section 4.9 ("Contours" tab) for controlling individual contour elements or displaying contours for a particular time interval.

\subsubsection{Displaying the Receptor Network}

The receptor field - where concentration and deposition values are calculated — can be viewed in the ArcMap map window by clicking on the checkbox labeled "Receptors" on the "Display Options" tab. In addition, the color, size, and other properties of the displayed receptors can be adjusted by clicking on the colored box next to the checkbox. 


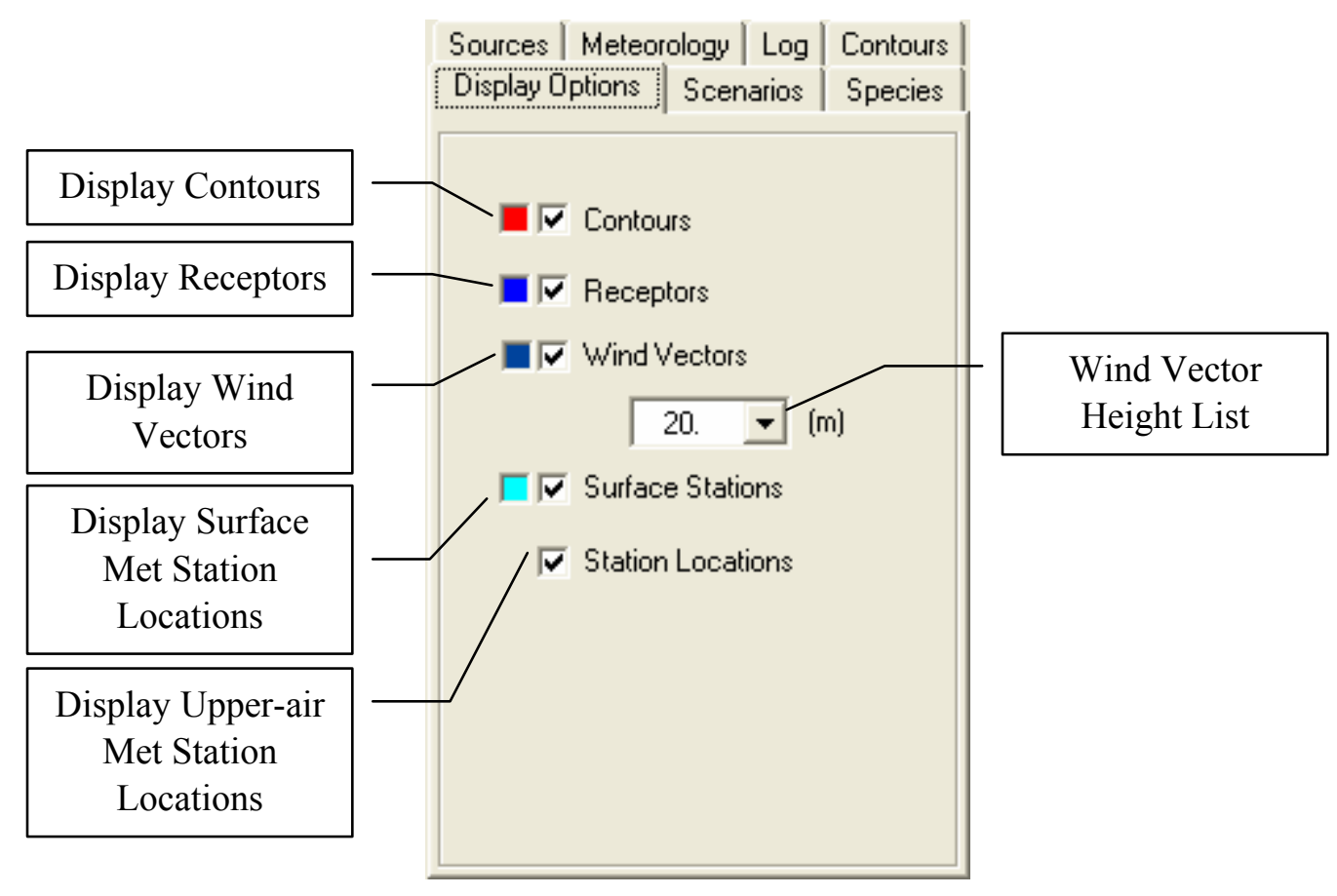

Figure 4.20. Display Options Tab

\subsubsection{Displaying the Calculated Wind Vector Field}

The gridded wind vector field created by the CALMET meteorological model and used in the dispersion calculations can be viewed in the ArcMap map window by clicking on the checkbox labeled "Wind Vectors" on the "Display Options" tab. Wind vectors can be displayed for various heights above the ground (in meters) by selecting a height from the listbox beneath the "Wind Vectors" label. In addition, the color, size, and other properties of the wind vectors can be adjusted by clicking on the colored box next to the checkbox. The number of wind vectors plotted and the size (i.e., scale) of the vectors can be adjusted within the Cal.par file. By default, wind vectors are displayed at every-other receptor location on the primary grid and are scaled such that a $3 \mathrm{~m} / \mathrm{s}$ wind is exactly the distance between successive receptor locations.

\subsubsection{Displaying Surface and Upper-Air Meteorological Station Locations}

Surface and upper-air meteorological stations that were used in the CALMET meteorological model for creating the gridded meteorological fields can be displayed in the ArcMap map window by clicking on the checkbox labeled "Surface Stations" and "Station Locations," respectively, on the "Display Options" tab. In addition, the color, size, and other properties of the stations can be adjusted by clicking on the colored boxes next to their respective checkboxes. 


\subsection{DUSTRAN Utilities}

A suite of utilities has been developed for DUSTRAN that automates certain data collection and assimilation processes. The datasets generated by these utilities can be used directly within the DUSTRAN interface and are intended to greatly extend the functionality of the modeling system while minimizing site-specific data-input requirements. Currently, two utilities-MetArchiver and Polygon Layer Creator-are available. MetArchiver downloads and archives surface and upper-air meteorological observations for direct use in a DUSTRAN simulation. The Polygon Layer Creator allows for the creation of site-specific characteristic files that can be used in certain DUSTRAN calculations. Layer Creator is currently used to create files containing gridded soil textures and vegetation codes that are used within the wind-blown dust emission module. This section provides documentation on how to use these utilities.

\subsection{The MetArchiver Utility}

The MetArchiver utility downloads and archives surface and upper-air meteorological observations in an Access database. The database is designed to be used in DUSTRAN and can be associated with a model simulation by selecting the "NOAA (Archived)" option on the "Meteorology" tab within DUSTRAN.

MetArchiver operates in two modes. The first mode is for managing the database that is used to store the downloaded meteorological data. The second mode is automated by the Windows' Task Scheduler and is used for routine download of surface and upper-air observations from two websites maintained by NOAA. Surface data are available for first-order National Weather Service (NWS) sites that record and transmit hourly observations; these data are publicly available on the web at: http://www.weather.gov/data/current_obs. Upper-air stations are for twice-daily (00Z and 12Z) radiosonde locations; these data are publicly available on the web through the Forecast Systems Laboratory (FSL) at: http://raob.fsl.noaa.gov.

MetArchiver is installed with DUSTRAN and is located in the root DUSTRAN directory (normally c:IDUSTRAN $\backslash$ MetArchiver.exe). Before scheduling the meteorological data download task (Section 5.1.2), the MetArchiver application will need to be configured for the desired stations and the creation of a station's database (Section 5.1.1). A continuous internet connection is required for observations to be accessed, updated, and stored locally in the database.

\subsubsection{Starting the MetArchiver Application}

To start the MetArchiver utility, double-click on the MetArchiver.exe file located in the root DUSTRAN directory. Once the utility has been started, a selection window will be displayed providing the following five options. Figure 5.1 displays an example of the options window. Each of the options is discussed in the sections that follow. 


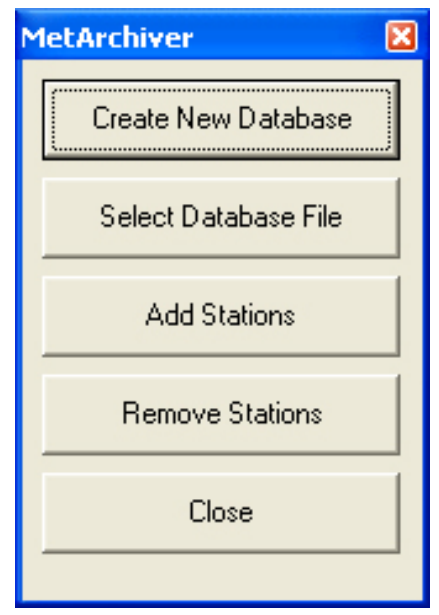

Figure 5.1. Example MetArchiver Options Window

\subsubsection{Create New Database}

The "Create New Database" option is used to create a new database file that can be used to store the meteorological data downloaded by the MetArchiver utility. To create a new database file:

- Click on the "Create New Database" button found on the options window.

- Enter a name for the new database into the database name field.

- Type in the directory path for the file or use the "Browse" button to select the directory.

- Click on the "Create" button.

Once the "Create" button has been clicked, the utility will create a new database file at the location specified by the user. During the creation process, the utility will automatically generate all required tables and fields needed to store the downloaded meteorological data as well as the tables used for selecting stations. Figure 5.2 displays an example of the "Create New Database" window.

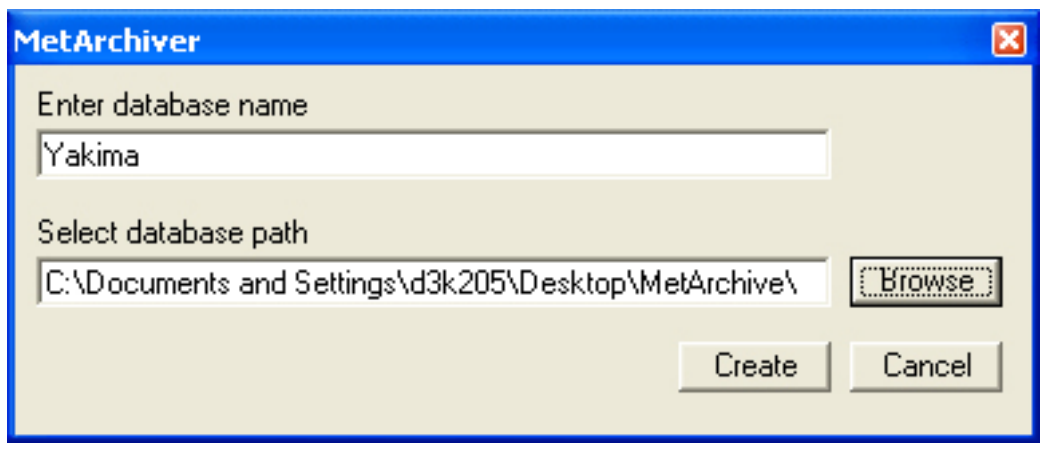

Figure 5.2. Example “Create New Database” Window 


\subsubsection{Select Database File}

The "Select Database File" option can be used to link an existing database file with the MetArchiver utility. To select an existing database file:

- Click on the "Select Database File" button found on the options window.

- Type in the full path, including the file name of the desired database file, or select it using the "Browse" option.

- Click on the "OK" button to finish the selection process.

Once a database file has been selected, the software will perform a set of checks against the database file to verify that the required tables and fields are present. If the selected file passes the checks, the system will configure itself to use the selected database for any future data downloads. Figure 5.3 displays an example of the "Select Database File" window.

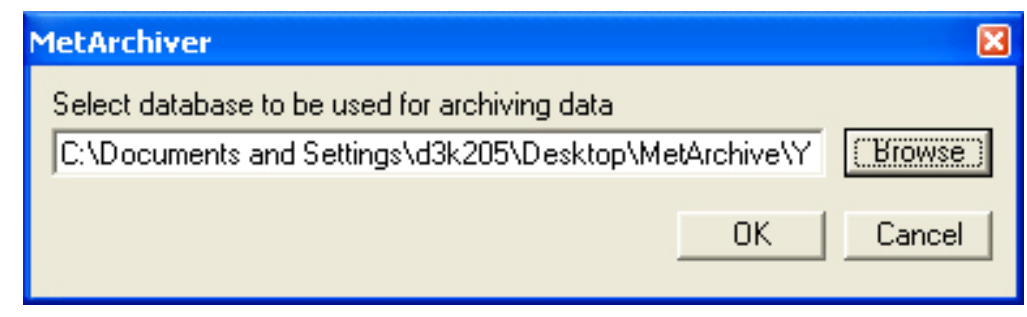

Figure 5.3. Example "Select Database" Window

\subsubsection{Add Stations}

The "Add Stations" option can be used to add new surface and upper air observation stations to the download list. To add new stations to the list:

- Click on the "Add Stations" button on the options window.

- Click on the "Select Station Type" control to select "Surface" or "Upper Air," depending upon the station type you would like to add.

- Select the state where the stations(s) are located on the "Select State" control.

- Select the desired stations from the displayed station list by checking the box next to the station ID and location.

- When the desired stations have been checked, click on the "Add" button to add the stations to the download list.

- Once all stations have been added to the download list, click on the "Done" button to return to the options window.

Figure 5.4 displays an example of the "Add Stations" window. 


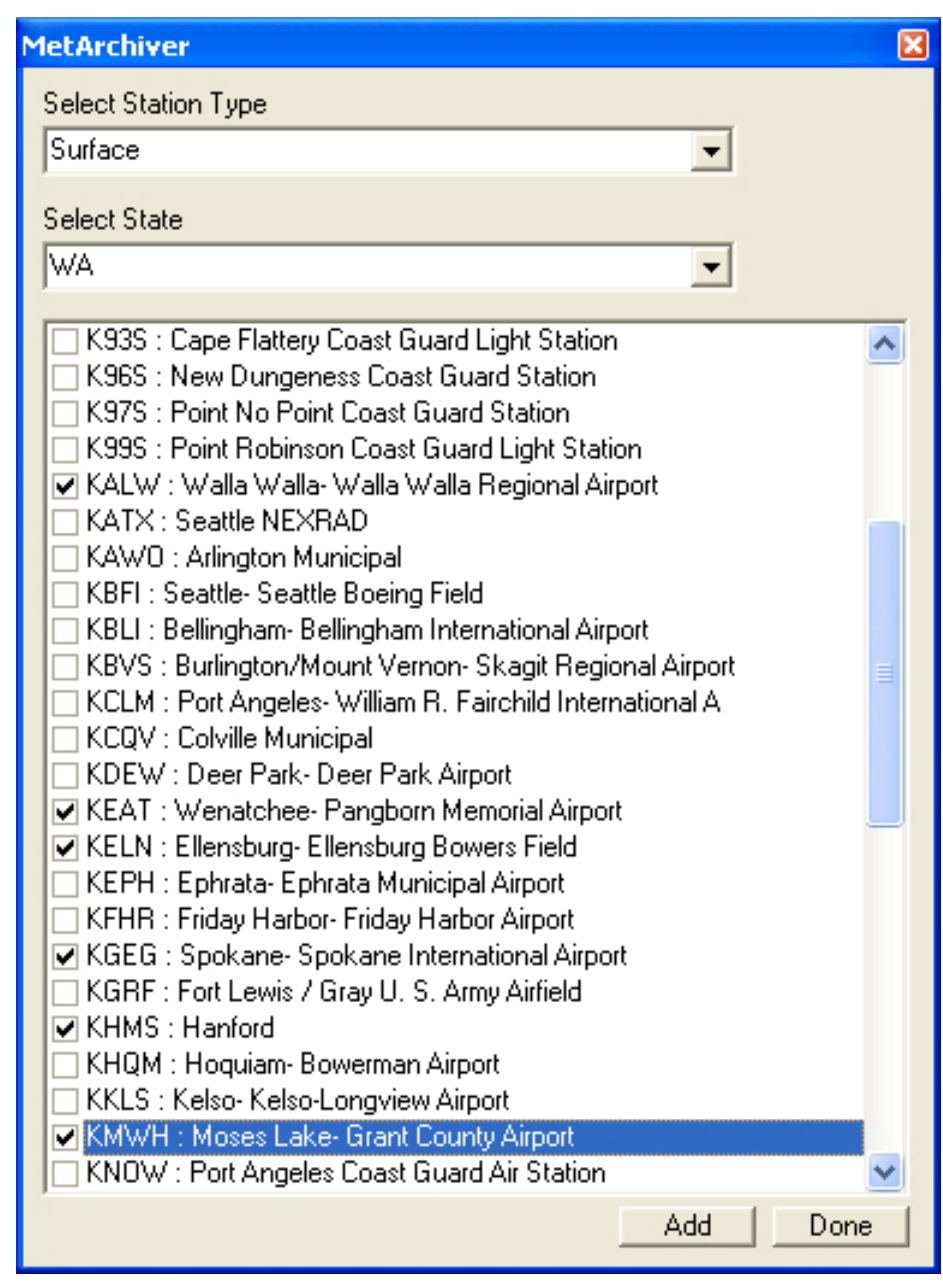

Figure 5.4. Example “Add Stations" Window

\subsubsection{Remove Stations}

The "Remove Stations" option can be used to remove stations from the download list. Please note that removing stations from the download list will not remove any data that have been previously archived for those stations. To remove stations from the download list:

- Click on the "Remove Stations" button on the options window.

- Using the "Surface stations" and "Upper air stations" lists, select the stations to be removed from the download list.

- Click on the "Remove" button to remove the stations and return to the options window.

Figure 5.5 displays an example of the "Remove Stations" window. 


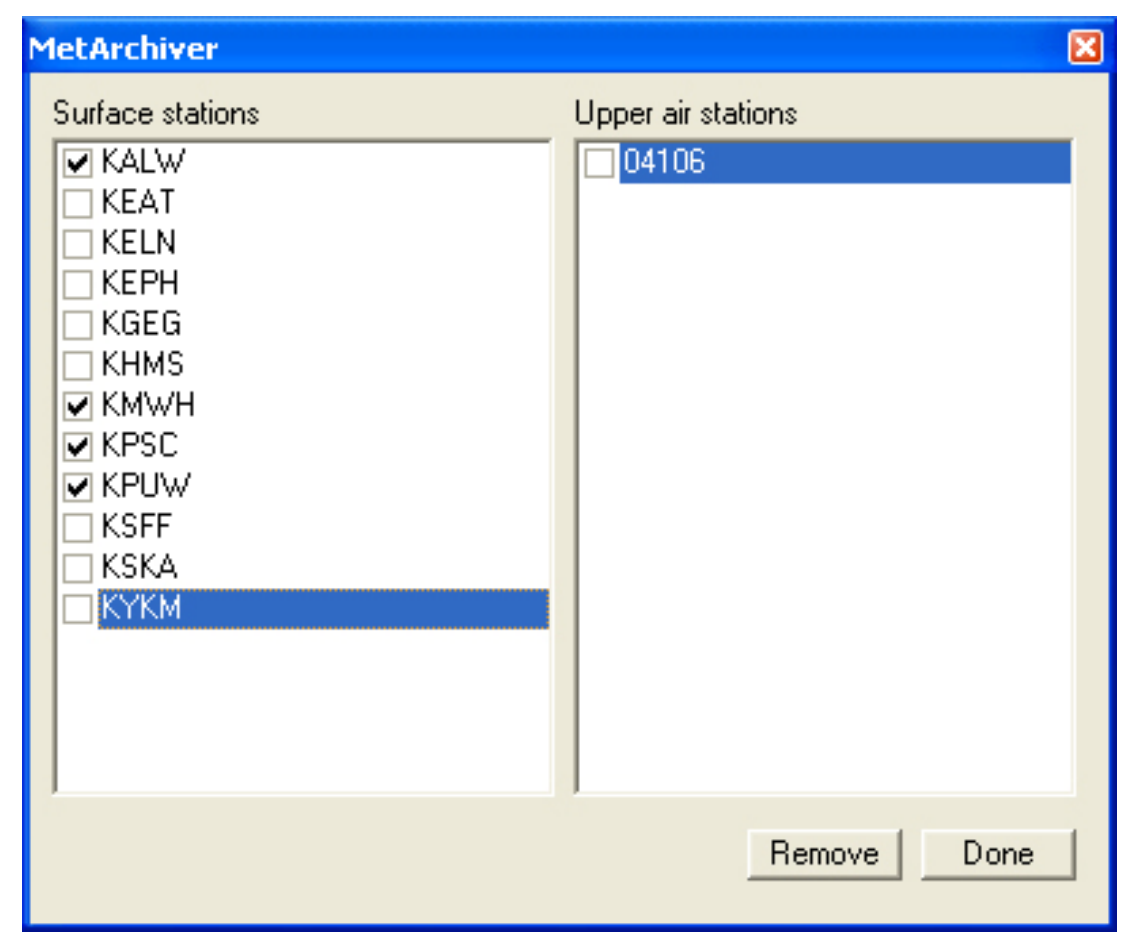

Figure 5.5. Example "Remove Stations" Window

\subsubsection{Closing the MetArchiver Utility}

The MetArchiver utility can be closed by clicking on the "Close" button of the options window or by clicking on the application terminate button located in the upper right-hand corner of the MetArchiver options window.

\subsubsection{Configuring MetArchiver with Windows Task Scheduler}

For the MetArchiver to automatically download surface and upper-air observations to the database for use in DUSTRAN, it needs to be configured with the "Task Scheduler" of the Windows operating system. The MetArchiver can be configured in the Window Task Scheduler using the following steps:

1. Select the "Add Scheduled Task" option from the "Scheduled Tasks" menu in the "Control Panel" of the Windows "Start" menu.

2. Once the "Scheduled Task Wizard" is displayed, click on the "Next" button.

3. Navigate to the MetArchiver.bat file that was included in the MetArchiver installation and select it using the "Browse" option of the wizard. This file normally resides in the root DUSTRAN directory.

4. Under the "Perform this task:" heading, select the "Daily" option and click the "Next" button.

5. Click the "Next" button on the time select window. Enter user name and password information. Click the 'Next' button to continue.

6. Click on the "Open advanced properties" check box to select it and click the "Finish" button.

7. Click on the "Schedule" tab of the advanced window. Click on the "Advanced" button. Select the "Repeat task" option on the "Advanced Schedule Options." Set the "Every" option to 1 hours and set the "Duration" option to 23 hours. 
8. Click the "OK" button to finish the Task Scheduler.

\subsection{Polygon Layer Creator Utility}

The Polygon Layer Creator utility is a software application that can be used to create or modify vegetation cover and soil texture GIS layers for wind-blown dust calculations. The utility uses the GIS to create areas (GIS "polygons") with specific vegetation or soil texture classes. Using the ArcMap .mxd project file for a DUSTRAN site, a user can draw areas onto the map of the site and assign characteristic codes to each of the polygon areas through simple point-and-click operations. Once new areas have been drawn or existing areas have been modified, the utility can be used to generate a new shape file containing the changes as well as a comma-delimited text (.csv) file. The text file can be used directly by the DUSTRAN modeling system for retrieving certain land-based characteristics, such as soil textures and vegetation classes, for use in wind-blown dust simulations. The interface for the Polygon Layer Creator utility consists of three parts: a map display, table of contents for the layers displayed in the map, and a toolbar with buttons for use in controlling the map display and editing the polygons. Figure 5.6 displays an example of the Polygon Layer Creator interface with a sample site .mxd file loaded.

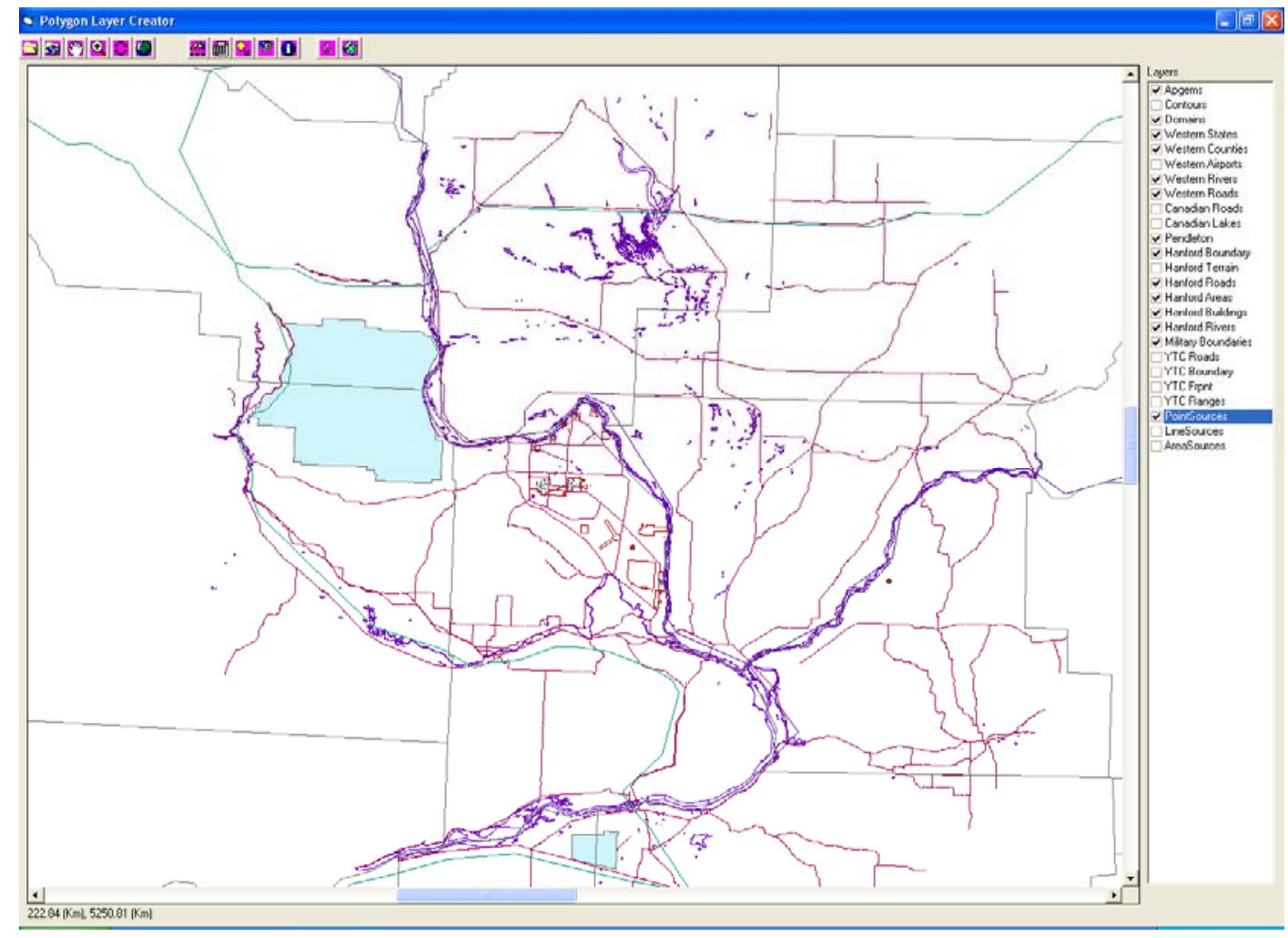

Figure 5.6. Polygon Layer Creator Interface

\subsubsection{Starting the Polygon Layer Creator}

The Polygon Layer Creator utility can be accessed from both within and outside of the DUSTRAN interface. To access the Polygon Layer Creator from within DUSTRAN: 
- Select the "Sources" tab and click on the "Characteristic Files" button. Characteristic files are comma-delimited text (.csv) files created by the Polygon Layer Creator utility that describe certain characteristics of the domain, such as soil textures and vegetation classes. These files can be read directly by the DUSTRAN interface for calculation purposes, such as calculating gridded dust emissions for the model domain.

- The "Characteristic Priorities" (Figure 5.7) form will appear, from which the "Polygon Layer Creator" button can be clicked to launch the application. The "Characteristic Priorities" form is used to associate the files created in Layer Creator with the current scenario.

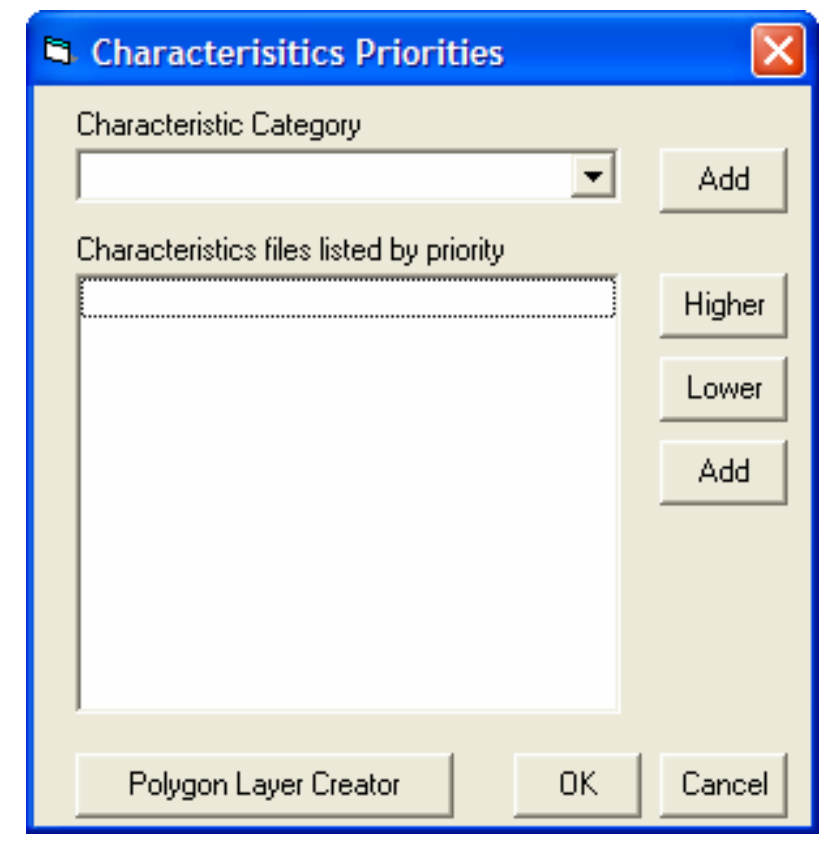

Figure 5.7. Launching Polygon Layer Creator from Within DUSTRAN

The Polygon Layer Creator utility can also be accessed outside of the DUSTRAN interface:

- To start the Polygon Layer Creator utility, double-click on the CreateLayer.exe file in the main DUSTRAN directory.

Once started, the application's interface will be displayed with all buttons disabled except for the

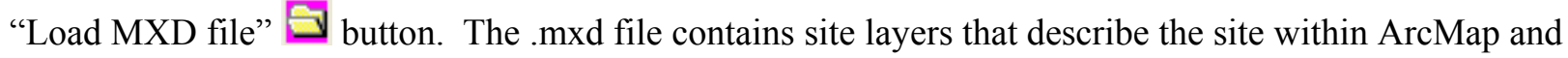
is normally generated by the "Add Site" wizard within DUSTRAN. The site .mxd file must be loaded before creating or editing site-specific polygons within the Layer Creator utility.

\subsubsection{Loading a Site's .MXD File}

An .mxd file contains layers that describe a site and is used to display the site within ArcMap. The .mxd file is normally created by the "Add Site" wizard within DUSTRAN; therefore, this file is usually located within the DUSTRAN directory for a given site. The .mxd file must be loaded before creating or editing site-specific polygons. To load a site's .mxd file: 
- Click on the "Load MXD file" $\square$ button.

- Using the browse window, navigate to the desired DUSTRAN site directory and select the .mxd file for that site.

- Click on the "Open" button of the browse window to open the .mxd file and close the browse window.

- Once the .mxd file has been selected, the map layers for the selected site will be automatically loaded into the map display, and the layer names will be displayed in the "Layers" list.

\subsubsection{Importing Polygons from an Existing Shape File}

Shape files can be loaded into the "Polygon Layer Creator" utility to quickly create polygon shapes for use in DUSTRAN. These shape files typically describe characteristic features for a site, such as soil textures or vegetation classes. To import polygons from a shape file:

- Click on the "Import polygons from shape file" button.

- Using the browse window, navigate to the directory folder that contains the desired shape file.

- Click on the shape file name and then click on the "Open" button to load the shape file and close the browse window.

- Once the shape file has been selected, a window will be displayed listing the field names available in the shape file.

- Select the primary field name that corresponds to the polygon values that will be used to describe the shapes. Figure 5.8 displays an example of the field selection window.

- Once selected, the shape file will be automatically loaded into the map display and its name added to the "Layers" list.

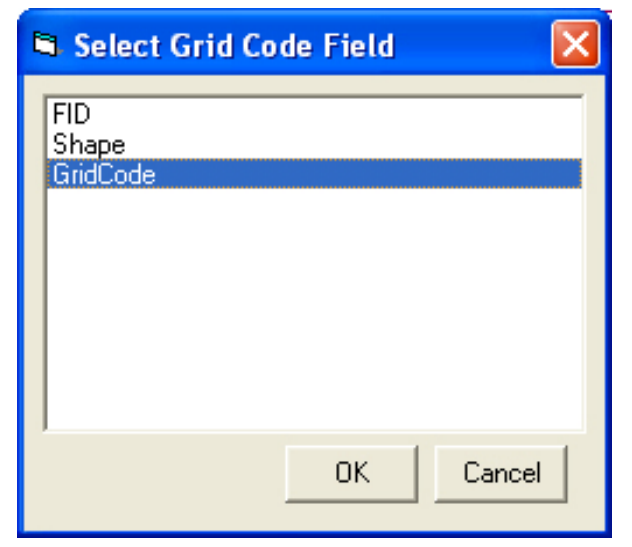

Figure 5.8. Select Grid Code Field Window

\subsubsection{Starting a New Polygon Set}

After a site's .mxd file has been loaded, polygons can be created to describe certain underlying characteristics of a site. To clear all existing polygons from a site and start a new polygon set: 
- Click on the "Start new polygon set" $\square$ button to clear the current set of polygons and start a new set.

- Once the "Start new polygon set" button has been clicked, the previously selected polygons will be cleared, and new polygons can then be entered.

\subsubsection{Site Navigation within Map Display Window}

Standard navigation buttons are included within the "Polygon Layer Creator" utility for working with a site in the map display window. These navigation features include:

- Panning

- Zooming

- Reverting to Prior Extent

- Viewing the Full Extent.

\subsubsection{Panning the Map Display}

Panning is used to move around a site without changing the zoom. To pan the view in the map display:

- Click on the "Pan map image" $\$$ button.

- Click and hold on a point in the viewable map display.

- Move the mouse, and this will then move the map view displayed in the map control.

\subsubsection{Zooming into the Map Display}

Zooming is used to focus on a particular location within a site. To zoom on the map display:

- Click on the "Zoom into map image" $\stackrel{\Theta}{ }$ button.

- Using the mouse, click on a point in the map display and then drag a rectangle around the area you would like to zoom into.

- Release the mouse button, and this will set the extent of the viewable map to the extent of the rectangle drawn, and the map will automatically zoom in.

\subsubsection{Reverting the Map Display to the Last Extent}

Reverting the map display to the last extent is used to undo a particular zoom level. To revert to the extent for the map display:

- Click on the "Zoom to last extent" $\widehat{N}$ button. The map display will automatically revert to the last extent used.

\subsubsection{Returning the Map Display to the Full Extent}

The full extent of the map display, as defined by the layers within the .mxd file, is an efficient way to quickly view the entire extent of the site. To view the full extent: 
- Click on the "Zoom to full map extent" button. The map display will automatically adjust to the full extent of the map and refresh the display.

\subsubsection{Working with Polygons}

The "Polygon Layer Creator" allows for the creation of polygon areas that spatially represent a certain characteristic, such as soil textures or vegetation classes, for a site. Each polygon is assigned a value, called a grid code, which identifies it from surrounding polygons. For example, soil textures may be assigned a grid code ID, which identifies it by Zobler soil category (see Table 2.4). These grid codes can be read by DUSTRAN and used in certain calculations. Currently, DUSTRAN can read gridded Zobler soil texture IDs and Olson Ecosystem vegetation codes (see Section 2.5.2), both of which are used in the wind-blown dust-emissions module. The "Polygon Layer Creator" utility creates gridded values of these codes from the polygon shapes that are displayed in the map window and writes them to a text file for use in DUSTRAN. This section provides guidance on how to work with polygon shapes within the Layer Creator utility.

\subsubsection{Adding Polygons to the Layer}

Polygons can be manually drawn and added to a layer to create a collection, or set of polygons. These polygons are used to represent a certain characteristic of the site, such as a specific soil texture or vegetation class. Polygons are differentiated by their grid code, which is a value that is entered after drawing the polygon shape. To add a new polygon to a polygon set:

- Click on the "Add polygons"

- Draw the new polygon onto the map display using the mouse. Each corner of the polygon is created by a left-click of the mouse at the desired location.

- When finished drawing the desired polygon, double-click on the last point of the polygon. An input window will be displayed asking for the code value associated with the polygon. Figure 5.9 displays an example of the input window.

- Enter the value into the input window and click on the "OK" button.

- The new polygon will then be added to the map display.

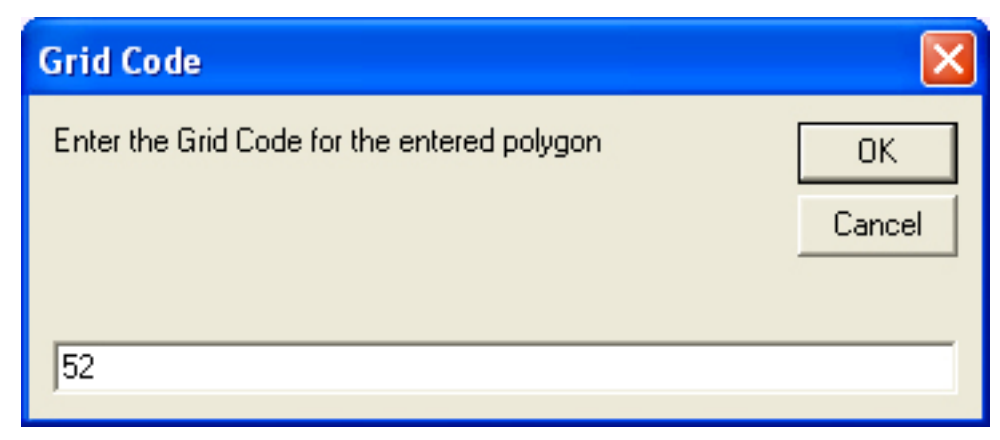

Figure 5.9. Grid Code Input Window 


\subsubsection{Editing the Value Associated with a Polygon}

Each polygon has an associated value which identifies a particular attribute of the polygon. These values are assigned at the time the polygon is created, but can be edited. To edit a value associated with a particular polygon:

- Click on the "Edit layer polygon" 盽 button.

- Double-click on the polygon whose value is to be changed.

- Once clicked, the polygon will flash, and an input window will be displayed showing the original value of the polygon.

- Enter the new value for the polygon and then select the "Ok" button.

\subsubsection{Deleting a Polygon}

A single polygon shape and its associated value can be deleted from the map display. To delete a polygon:

- Click on the "Delete polygon" $\nabla_{x}$ button.

- Double-click on the polygon to be deleted.

- Once clicked, the polygon will flash, and then a message window will be displayed asking for confirmation of the deletion.

- Click on the "OK" button to delete the polygon.

\subsubsection{Changing the Color of the Polygon Boundaries}

The boundaries that define a polygon area can have different colors to distinguish it from other polygons within the map display. Normally, colors are used for display purposes and visually represent values used to define each polygon layer. To change polygon boundary colors:

- Click on the "Change the color of the polygon boundaries" button. Once the button has been clicked, the "Grid Code Color Select" window will be displayed. Figure 5.10 displays an example of the "Grid Code Color Select" window.

- Within the "Grid Code Color Select" window, double-click on the code value that the color will be changed. Once the code has been clicked, a color selection window will be displayed.

- Select the desired color by clicking on the colored box and then click on the "OK" button.

- Once the colors have been selected for all polygons, click the "Ok" button of the "Grid Code Color Select" window. The polygon boundary colors will be automatically updated in the map display. 


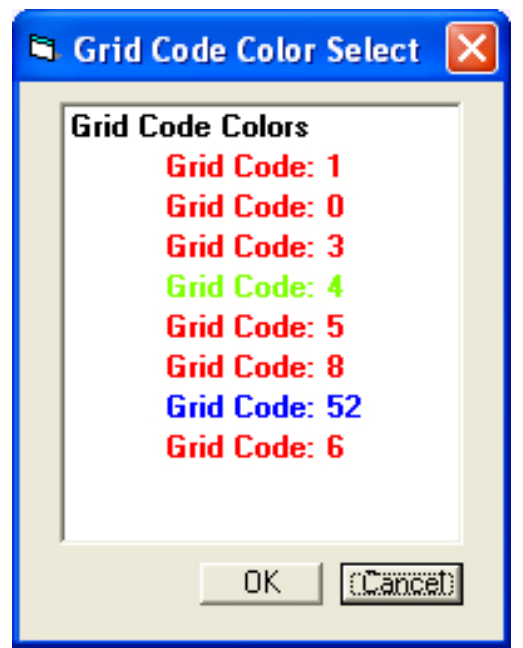

Figure 5.10. Grid Code Color selection Window

\subsubsection{Viewing the Value of a Polygon}

The grid code value that defines a polygon can be viewed within the map display window. To view the polygon value:

- Click on the "Show polygon grid code" button and then click on the polygon of interest.

- After clicking on a polygon, the polygon will flash, and the "Polygon Grid Code Value" window will be displayed with the value of the polygon. Figure 5.11 shows an example of the "Polygon Grid Code Value" window.

- To close the "Polygon Grid Code Value" window, click on the "OK" button.

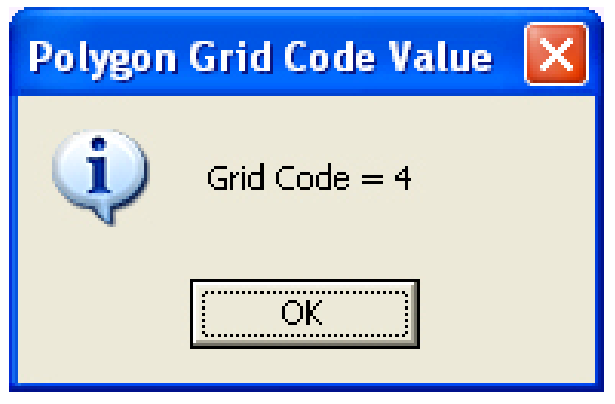

Figure 5.11. Polygon Grid Code Value Window

\subsubsection{Setting the Resolution of the Output .csv File}

The polygon areas and their associated values output a comma-delimited text (.csv) file that can be read by DUSTRAN and used in certain calculations. Currently, DUSTRAN can read gridded Zobler soil texture IDs and Olson Ecosystem vegetation codes, both of which are used in the wind-blown dust gridded emissions module. To set the resolution, or grid spacing, derived from the polygon shapes: 
- Click on the "Modify the resolution of the output data" button; the "Specify Sampling Resolution" window will be displayed (Figure 5.12).

- Enter the desired "Easting" and "Northing" resolutions into the text boxes provided.

- Click on the "OK" button to accept the changes and close the window.

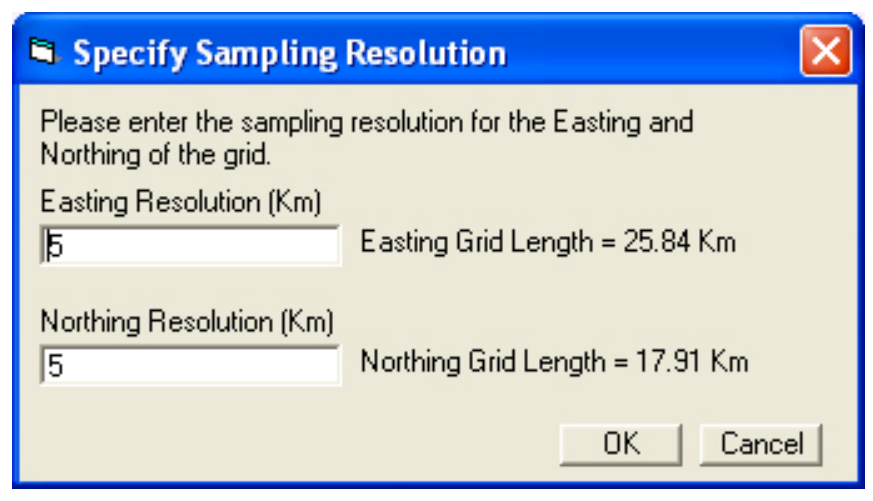

Figure 5.12. Specify Sampling Resolution Window

The output grid resolution should be commensurate with the average size of the polygons making up the data set. Fore example, if the various polygons making up the data set have an average dimension of roughly $10 \mathrm{~km}$ on a side, then the grid resolution should be on the order of $5 \mathrm{~km}$ to have roughly four grid points per polygon.

\subsubsection{Creating the Output Shape File and .csv File}

The polygons can be saved to a shape and text file for use in DUSTRAN. The shape file allows for the polygons to be displayed within the DUSTRAN map window; the text file can be interrogated by the DUSTRAN software for use in certain calculations. Currently, DUSTRAN reads gridded Zobler soil texture IDs and Olson Ecosystem vegetation codes, both of which are used in the wind-blown dustemissions module. To create the files, click on the "Create shape file" button; a file browse window will be displayed. Enter a name for the output files and then click on the "Open" button to save the files. 


\subsection{Adding a New Site to DUSTRAN}

A new site can be added to DUSTRAN using the "Add Site" wizard within the DUSTRAN interface. A new site contains all the geophysical data, such as terrain and land use/land cover, that is needed to run the CALMET, CALPUFF, and CALGRID models. In addition, a new site contains base geospatial data that are used for displaying and analyzing the site within ArcMap. After a new site has been created, the user can add additional geospatial data to customize the map for a particular scenario or application.

This section provides step-by-step instructions for adding a new site to DUSTRAN. Specifically, the "Add Site" wizard will guide the user through a series of forms that automate the following tasks:

- Specify the spatial range, or extent, of the new site.

- Designate a name and UTM zone for the site.

- Create a new site directory that contains all the necessary template files for performing a simulation in DUSTRAN.

- Create and load a new, personal geo-database that contains standard geospatial data for display in ArcMap.

- Build DUSTRAN's meteorological surface (.snf) and upper-air (.unf) station information files.

- Build and save a new map file (.mxd) for display in ArcMap.

- Register the site so that it can be opened within the DUSTRAN interface.

1. To add a new site, both ArcMap and DUSTRAN must already be installed. Open ArcMap and click on the "DUSTRAN" button on the ArcMap toolbar. DUSTRAN will open as a console application within ArcMap.

2. Within DUSTRAN, click on the "Add Site" button (see Figure 6.1):

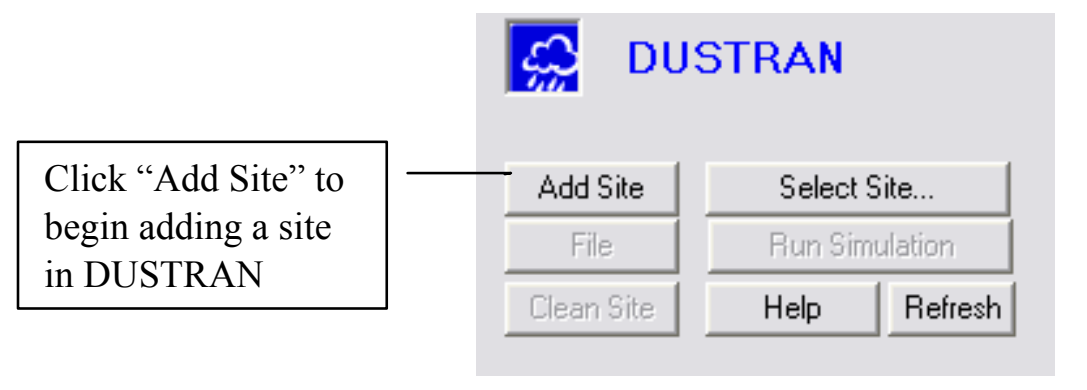

Figure 6.1. DUSTRAN's “Add Site” Button

3. After clicking "Add Site," a set of instructions will appear that provides information on how to select the site location within ArcMap (see Figure 6.2). After reading the instructions, click "OK" to continue. 


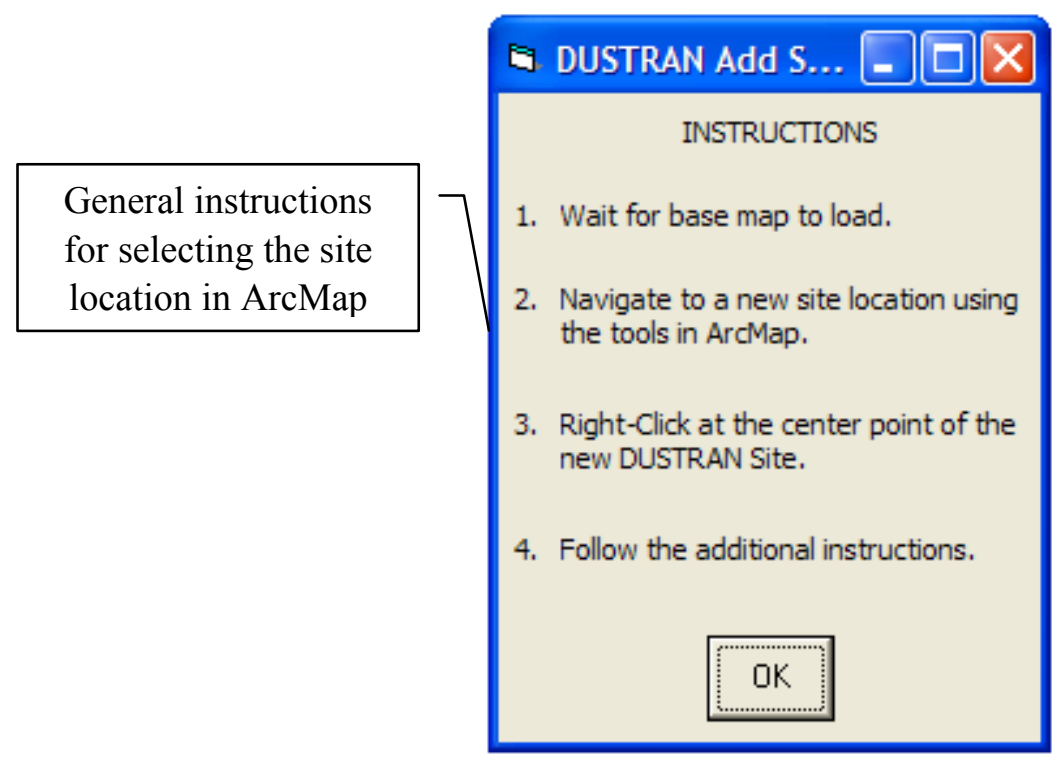

Figure 6.2. DUSTRAN's Instructions for Selection of Site Location

4. Use the ArcMap navigation tools (e.g., zoom and pan) to navigate to a desired location on the base map where the new site is to be located. Right-click on the general location of where the site is to be located. This sets a central point from which all geospatial data will be selected and clipped.

5. After right-clicking on the map, a dialog box will appear prompting for the geospatial site dimensions (see Figure 6.3). This affects the overall extent of the geospatial data and sets the clipping envelope for the data. The default values for the height and width are $600 \mathrm{~km}$ to verify that the spatial envelope is large enough to provide flexibility in siting the model domain in DUSTRAN. After setting the height and width for the geospatial envelope, click "OK" to continue.

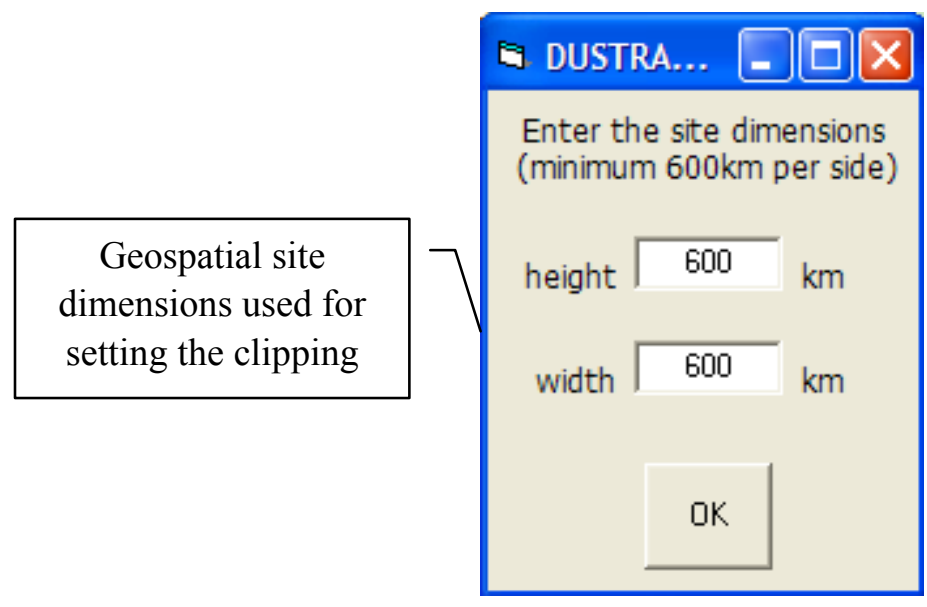

Figure 6.3. Prompt for Geospatial Site Dimensions

6. The base map will be displayed with a red box that represents the clipping envelope that will be applied to the geospatial data (see Figure 6.4). The box is used to intersect the "US_States" layer. 
The envelope of the intersecting states serves as the actual clipping boundary for the geospatial data. Click "Yes" to accept or "No" to start again.

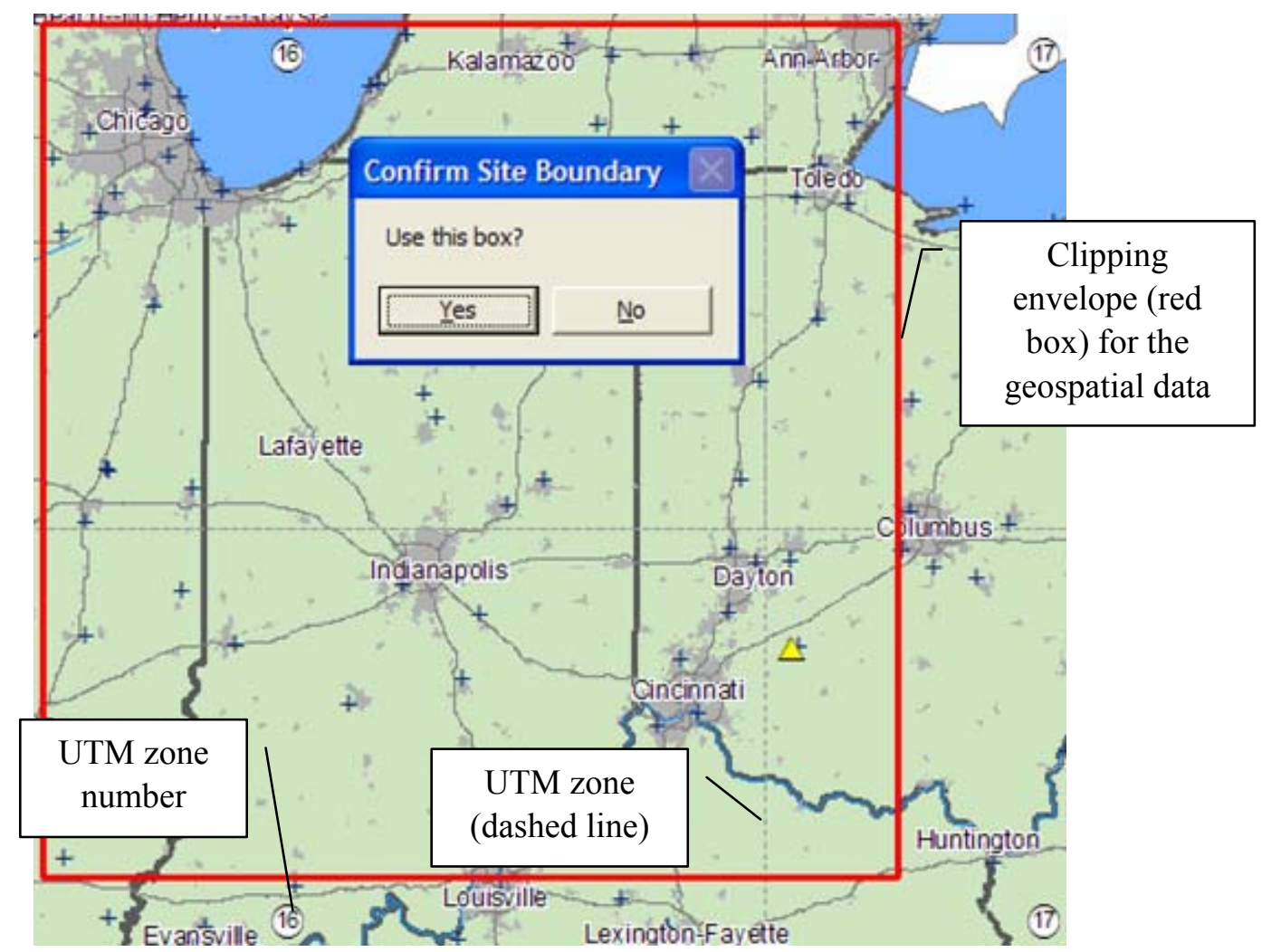

Figure 6.4. Red Box Enclosing Clipping Envelope

7. After accepting the clipping envelope, a dialog box will appear prompting for the site name and UTM zone (see Figure 6.5). The site name must be unique and cannot be the name of an existing site. The UTM zones are labeled on the base map (see image above for an example) and are used as the coordinate system in DUSTRAN. If the center of the site is in a UTM zone that is different than the UTM zone selected by the user, the selection must be confirmed before continuing.

8. Click "Finish" to finalize the site creation process. A progress bar and task list display the progress of the site creation. Because the utility must clip all of the base layers, loading the geo-database may take several minutes, especially for sites located on the eastern coast of the United States.

9. A window will appear asking for the sampling resolution for the vegetation and soil texture files that are used in wind-blown dust simulations (see Figure 6.6). A default resolution of $4 \mathrm{~km}$ has been set.

10. Click the "OK" button, and the wizard will create four files: srztext.shp, srztext.csv, owe14d.shp, and owe14d.csv. The srztext files define the soil texture, and the owe14d files define the vegetation cover. The files with the extension .shp are shape files that are viewable within ArcMap and are stored within the site's geodatabase; the .csv files are comma-delimited ASCII files that are interrogated by DUSTRAN when performing a wind-blown dust simulation and are stored within the TerData directory of the new site. 


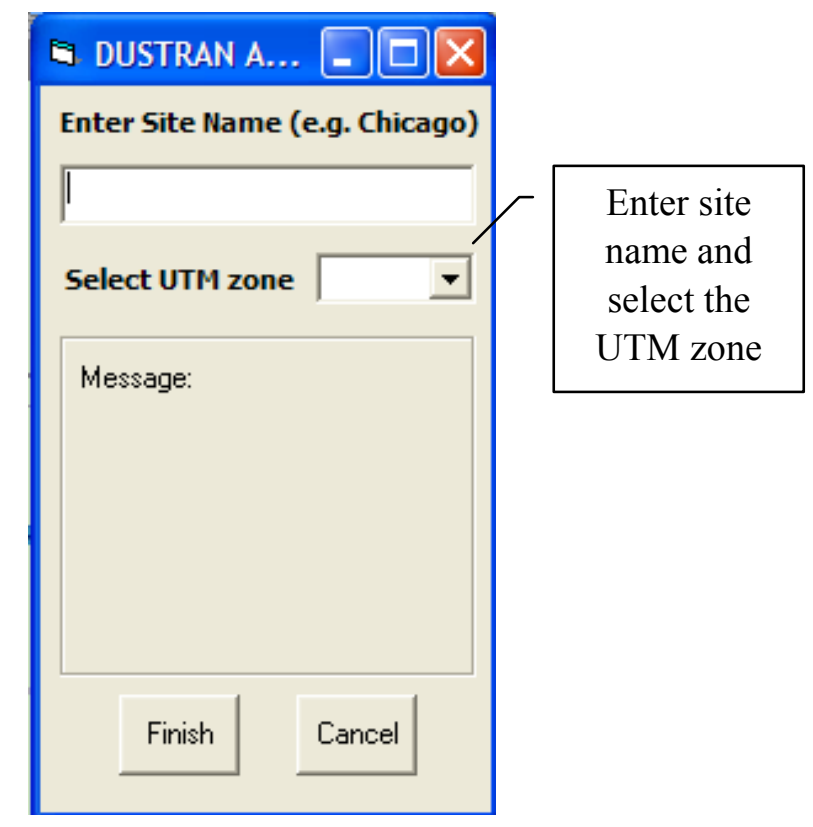

Figure 6.5. Prompt for Site Name and UTM Zone

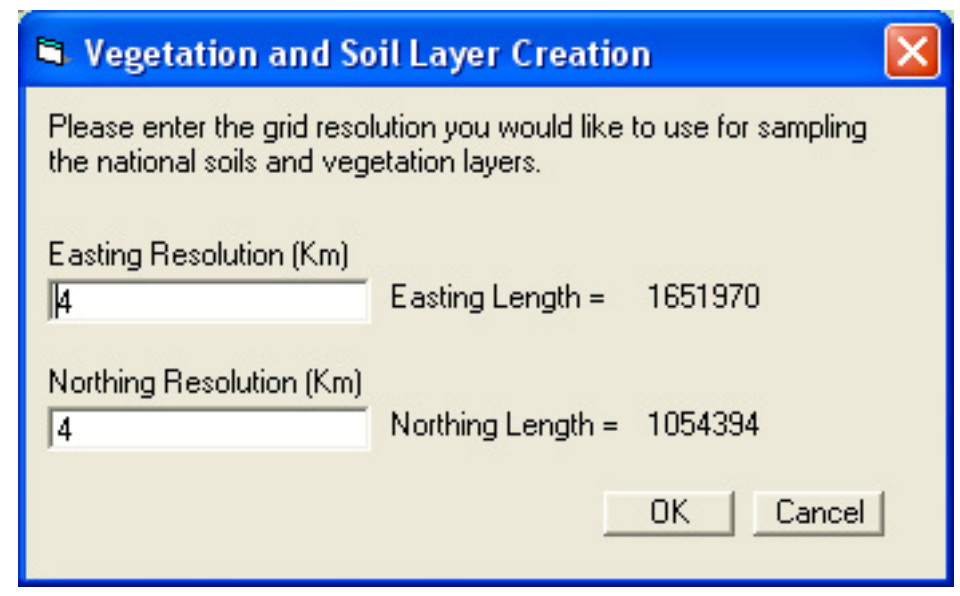

Figure 6.6. Prompt for Sampling Resolution

11. Following the site's registration, a new map file is constructed for the site. The file is automatically symbolized, displayed, and saved. The site will close, and ArcMap may prompt and ask if the user wishes to save the changes to the base map (Addsite.mxd). Select "NO," as the base map is used for the creation of future sites. The new site is displayed, and the user is notified with a message box to access the new site from the "Select Site" button within the DUSTRAN interface (see Figure 6.7). 


\begin{tabular}{|c|c|c|}
\hline Ddd Site & \multicolumn{1}{|c|}{$\begin{array}{c}\text { After the } \\
\text { site has been } \\
\text { created, it is } \\
\text { available for } \\
\text { use in } \\
\text { DUSTRAN }\end{array}$} \\
\hline File & Pelect Site... \\
\hline Clean Site & Help & Refresh \\
\hline
\end{tabular}

Figure 6.7. Notification for Accessing the New Site 


\subsection{DUSTRAN Example Tutorials}

This section provides example tutorials for creating and running DUSTRAN simulations. Two tutorials are provided, both based on the sample Yakima site (Note: the Yakima site can be installed from the DUSTRAN installation CD; see Section 3.2, Installing a Site). The first tutorial simulates dust emissions from a point and area source. The point-source emissions are prescribed explicitly; the areasource emissions are from vehicular activity and use the vehicular dust-emissions module intrinsic to DUSTRAN to generate the particulate emission rate. The second tutorial simulates a wind-blown dust event using the wind-blown dust-emissions module within DUSTRAN.

To run this example, ArcMap, DUSTRAN, and the Yakima site must already be installed. Refer to the section entitled "DUSTRAN Installation Instructions, Section 3.0" in this manual for detailed instructions on installing DUSTRAN and the Yakima site.

\subsection{Simulating Dust Dispersion from Source Emissions}

This tutorial steps through a dust-dispersion scenario from a point and area source. The point source represents particle emissions from a stack, and the emissions are defined explicitly. The area source represents a region of dust emissions from vehicular activity; these emissions are calculated automatically by the DUSTRAN vehicular dust-emissions module. Both sources are set to run for the same duration, and the downwind air-concentration and soil deposition are simulated.

\subsubsection{Starting DUSTRAN}

DUSTRAN is an integrated dispersion modeling application within the ArcMap (Version 9.x) GIS interface. To begin a DUSTRAN simulation, open ArcMap. On the ArcMap toolbar, click on the "DUSTRAN" button (see Figure 7.1):

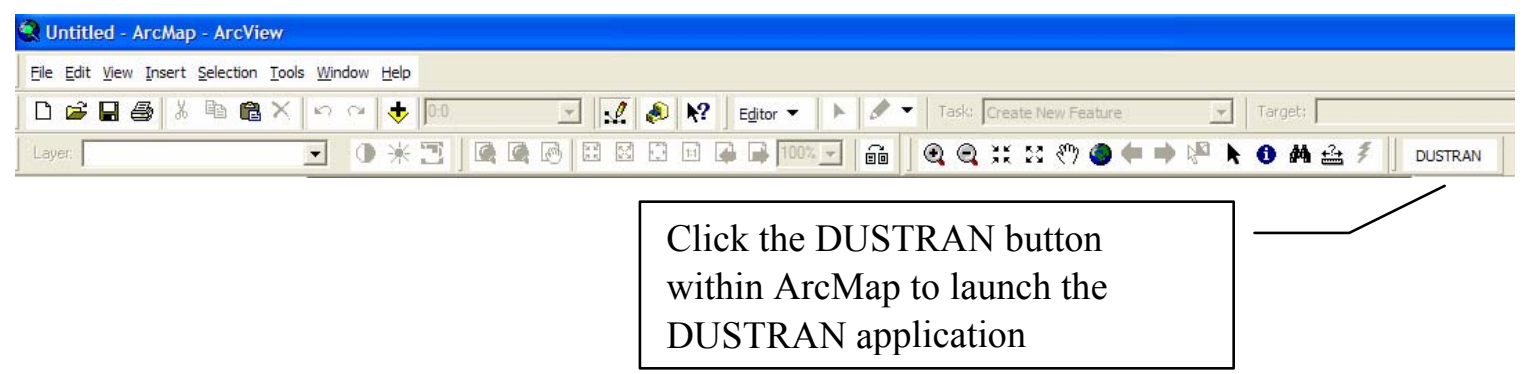

Figure 7.1. ArcMap Toolbar

\subsubsection{Selecting a Site}

The user interface to the DUSTRAN model will appear in a frame within the ArcMap application (see Figure 7.2). Click the "Select Site..." button to open a dialog box that allows the user to select an existing site: 


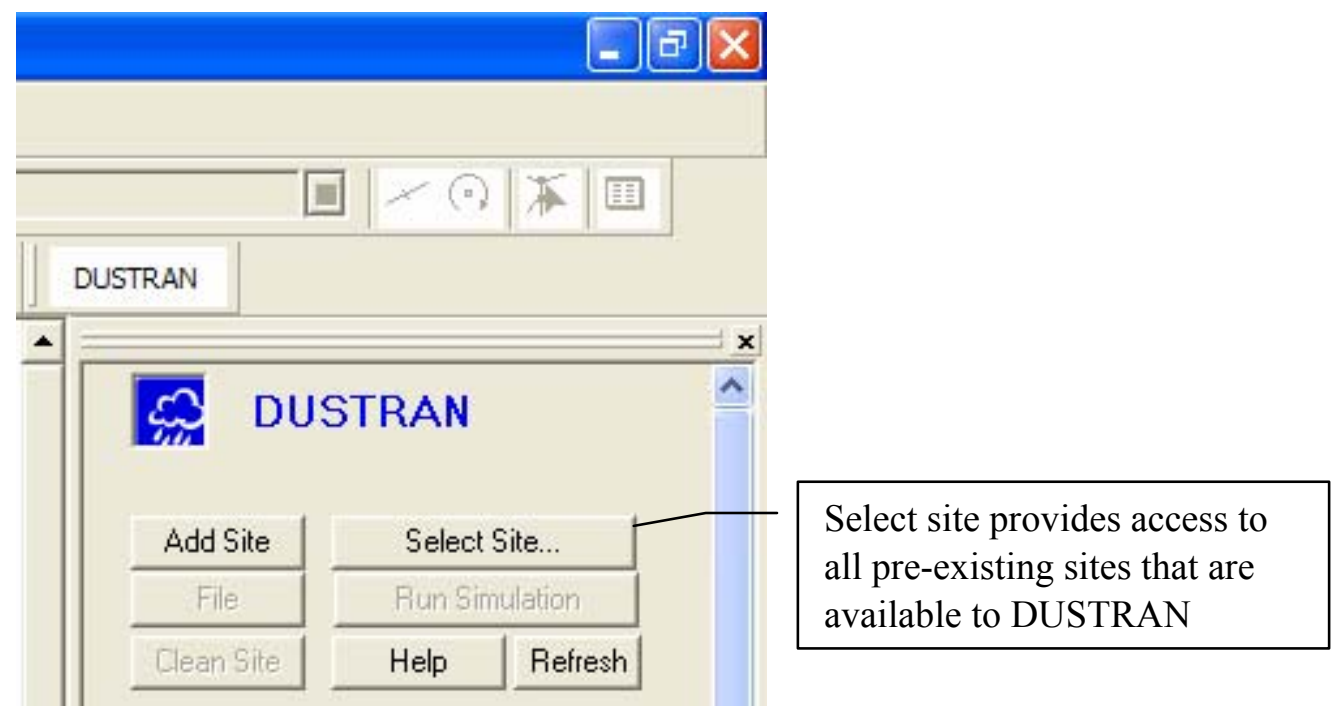

Figure 7.2. DUSTRAN User Interface

Select "Yakima" from the list of available model sites (see Figure 7.3) and then click "Open":

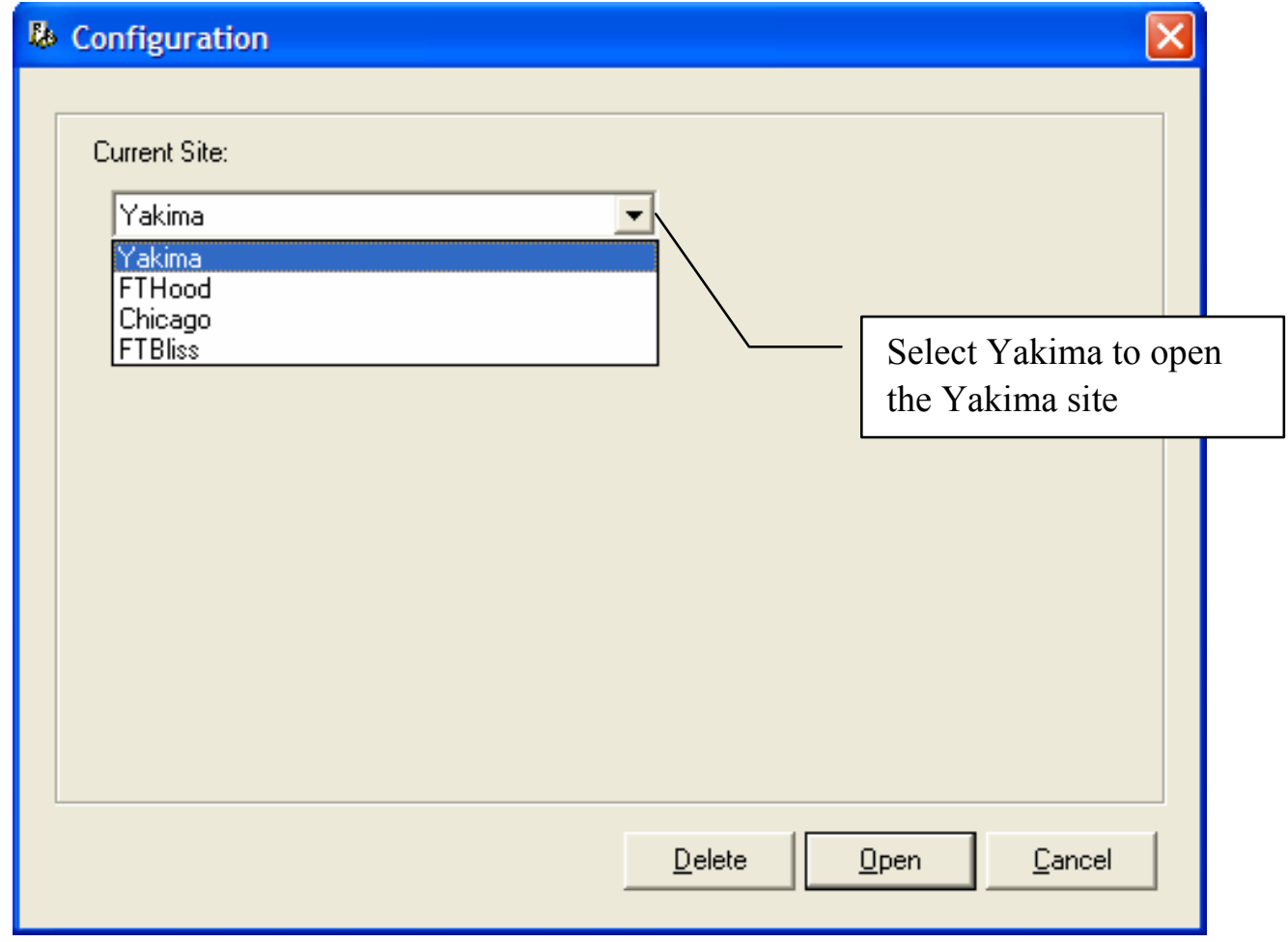

Figure 7.3. Available Model Sites

The Yakima site will open within ArcMap. A list of available GIS data layers will appear in the left frame, and DUSTRAN-specific input parameters will appear in the right frame (see Figure 7.4). 


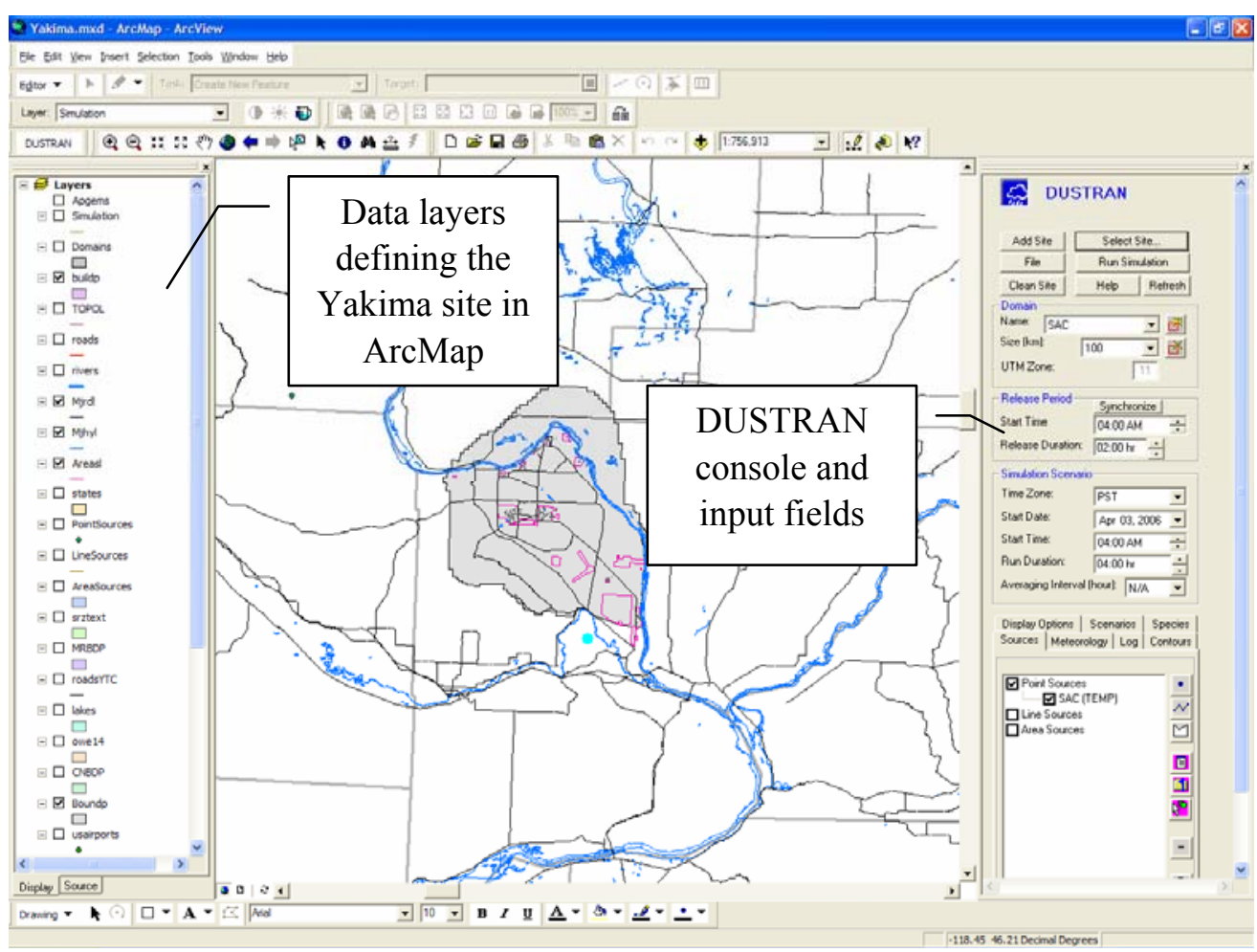

Figure 7.4. Yakima Site Displayed

\subsubsection{Creating a Domain}

The first step in setting up a scenario in DUSTRAN is to create a domain and set the domain size. A domain is a user-specified area where both meteorological and dispersion model calculations are performed. Click the "New Domain" button in the "Domain" panel (see Figure 7.5). Then, click on a location (somewhere near the center of the site) in the map window that represents the center of the model domain. In the dialog box that appears, enter the name "Yakima" for the domain and click "OK."

By default, the domain size is set to $100 \mathrm{~km}$. Set the size from the "Size" listbox in the "Domain" panel to $80 \mathrm{~km}$. The domain should now appear as a shaded, rectangular region bounding DOE's Hanford area.

\subsubsection{Setting the Model Specie}

By default, there are four PM species available to model in DUSTRAN (see Figure 7.6). This tutorial will model the 10 micron $\left(\mathrm{PM}_{10}\right)$ specie; uncheck $\mathrm{PM}_{2.5}, \mathrm{PM}_{15}$, and $\mathrm{PM}_{30}$. Additional species (particles and gases) can be added using the "Add Specie" $\boldsymbol{\Psi}$ button on the "Species" tab, but for this exercise, we will use the existing $\mathrm{PM}_{10}$ specie in the list: 


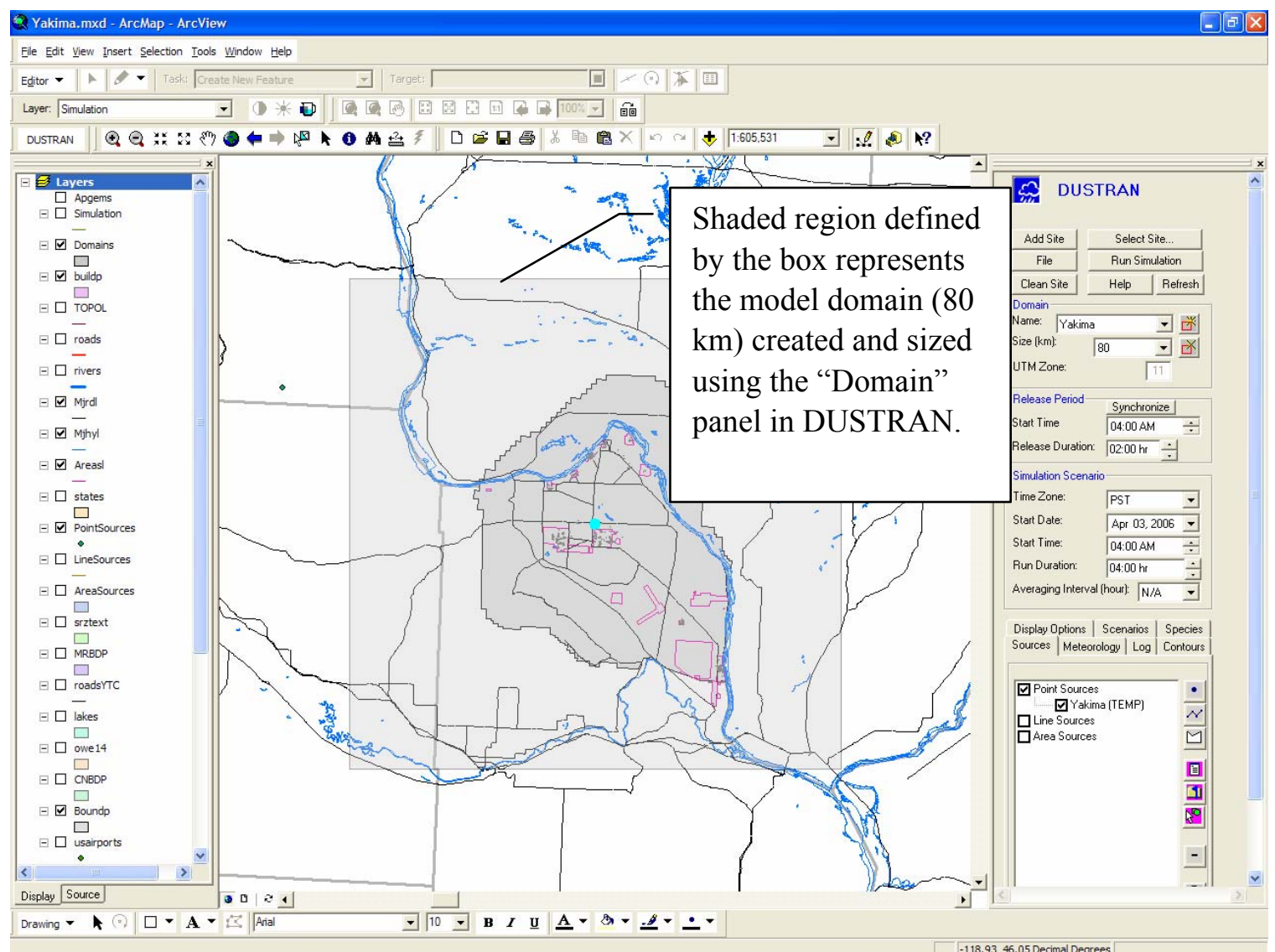

Figure 7.5. Domain Panel

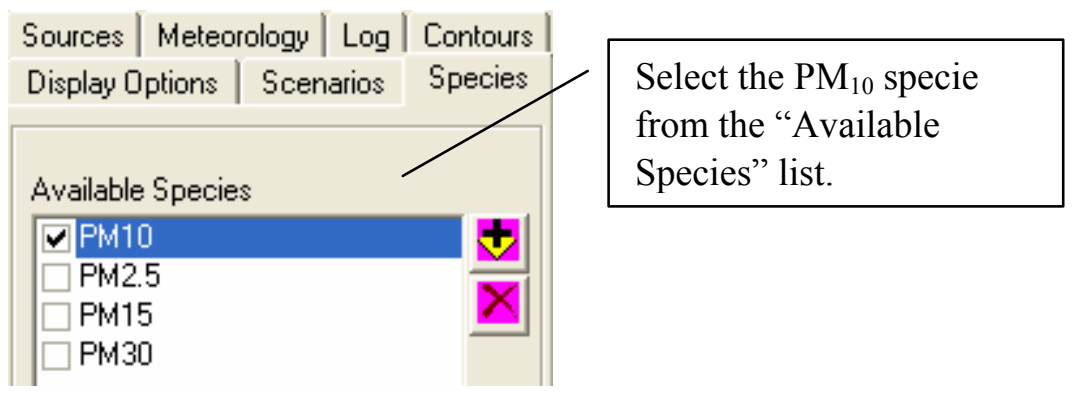

Figure 7.6. Available Species List

\subsubsection{Creating a Point Source}

Click on the "Sources" tab. Note that there is already a point source called "Yakima" listed under "Point Sources"; this corresponds to the point that is used by DUSTRAN to mark the center of the domain. Uncheck the name, and the point will disappear from the center of the domain in the ArcMap map window. 
To create a new point source, click on the "Point Source" - $\mid$ button on the "Sources" tab. Click on a location within the domain to place the point source. Call the source "Stack" and click "OK."

The "Source Input" form for the point source will appear (see Figure 7.7). Enter the "Release Parameters" as follows and click "OK" to continue:

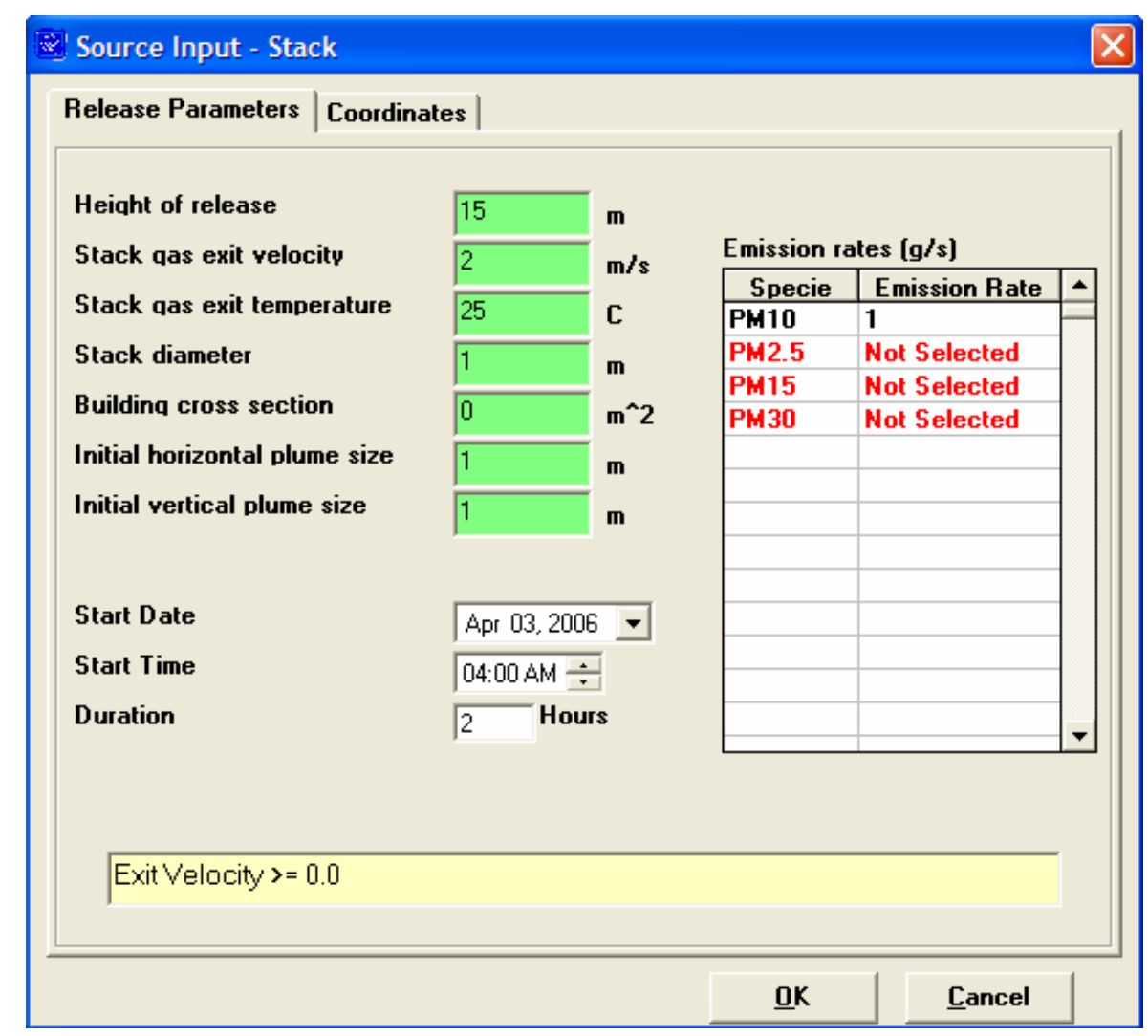

Figure 7.7. Point Source Input Form

Notice that the new source shows up as a point in the ArcMap map window and also appears in the "Point Source" list on the "Sources" tab.

\subsubsection{Creating an Area Source}

Next, create an area source by clicking on the "Area Source" $\square \mid$ button on the "Sources" tab. An area source can be a triangle or four-sided polygon. It is created by clicking on three or four locations depending on if want a triangle or a four-sided polygon in the ArcMap map window. The final corner should be a "double-click" to complete the polygon. Create the area source by drawing $\approx 1 \mathrm{~km}$ square polygon near the center of the domain, enter the source name "Field," and click "OK."

The "Source Input" form for the area source will appear (see Figure 7.8): 


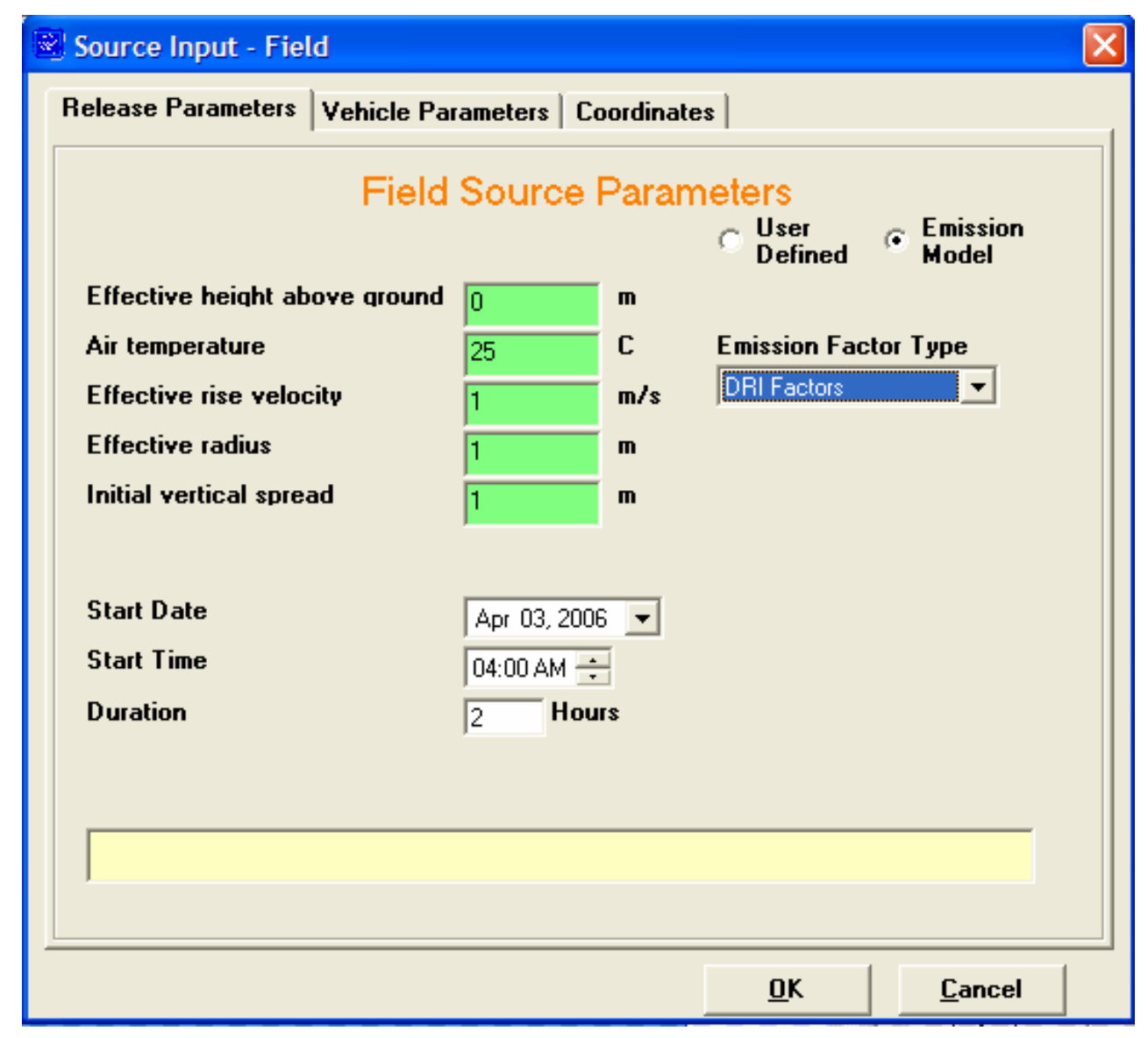

Figure 7.8. Area Source Input Form

For the area source, we will use the "Emission Model" to calculate dust emissions created by a single vehicle using the "DRI Factors" (Desert Research Institute). Vehicular dust emissions are a function of vehicle parameters, such as weight and speed. Click on the "Vehicle Parameters" tab (see Figure 7.9) and specify the following vehicular information:

- The "Distance Traveled" is the total distance traveled by the vehicle within the area. The emissions are assumed to be uniformly distributed over the area and constant for the duration of the release.

- Click "OK," and the area source will appear in the ArcMap map window and also appears under the "Area Sources" list on the "Sources" tab (see Figure 7.10).

\subsubsection{Entering Meteorological Data}

Next, click on the "Meteorology" tab within DUSTRAN and select "Single Observation" from the listbox. The "Specify Meteorological Data" form appears. Enter the meteorological parameters as shown in the form below. Select the "Atmospheric Stability" as "E - Slightly Stable," reasonable for early morning springtime conditions. Also, enter a wind direction from approximately the west-southwest (240 degrees). Figure 7.11 shows the completed form. 


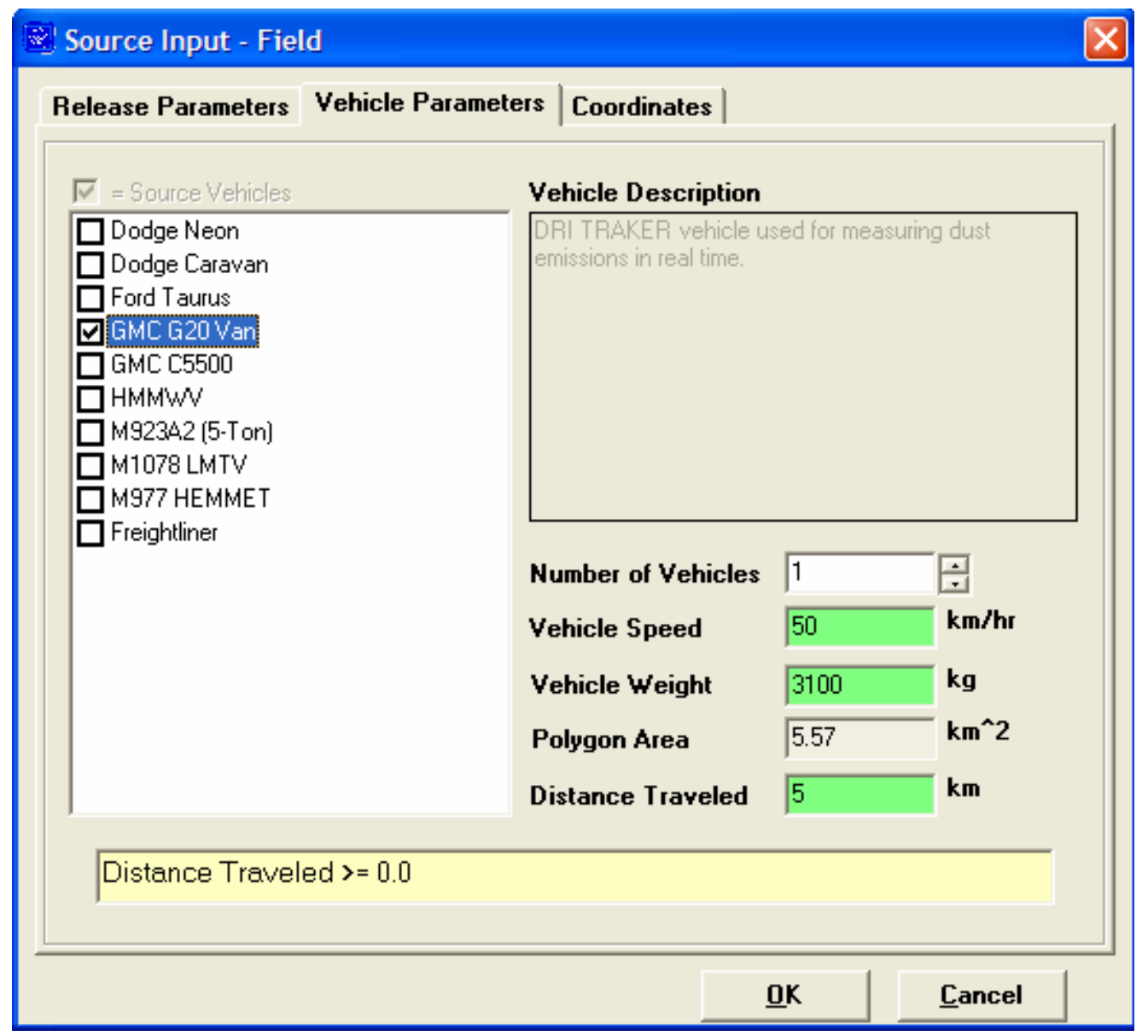

Figure 7.9. Information Required Under the Vehicle Parameters Tab

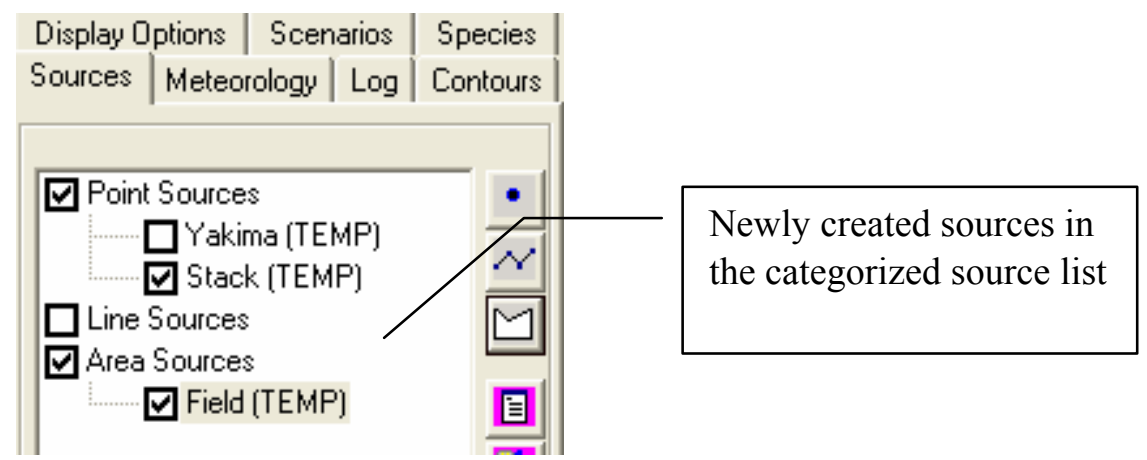

Figure 7.10. Area Sources List on the Sources Tab 


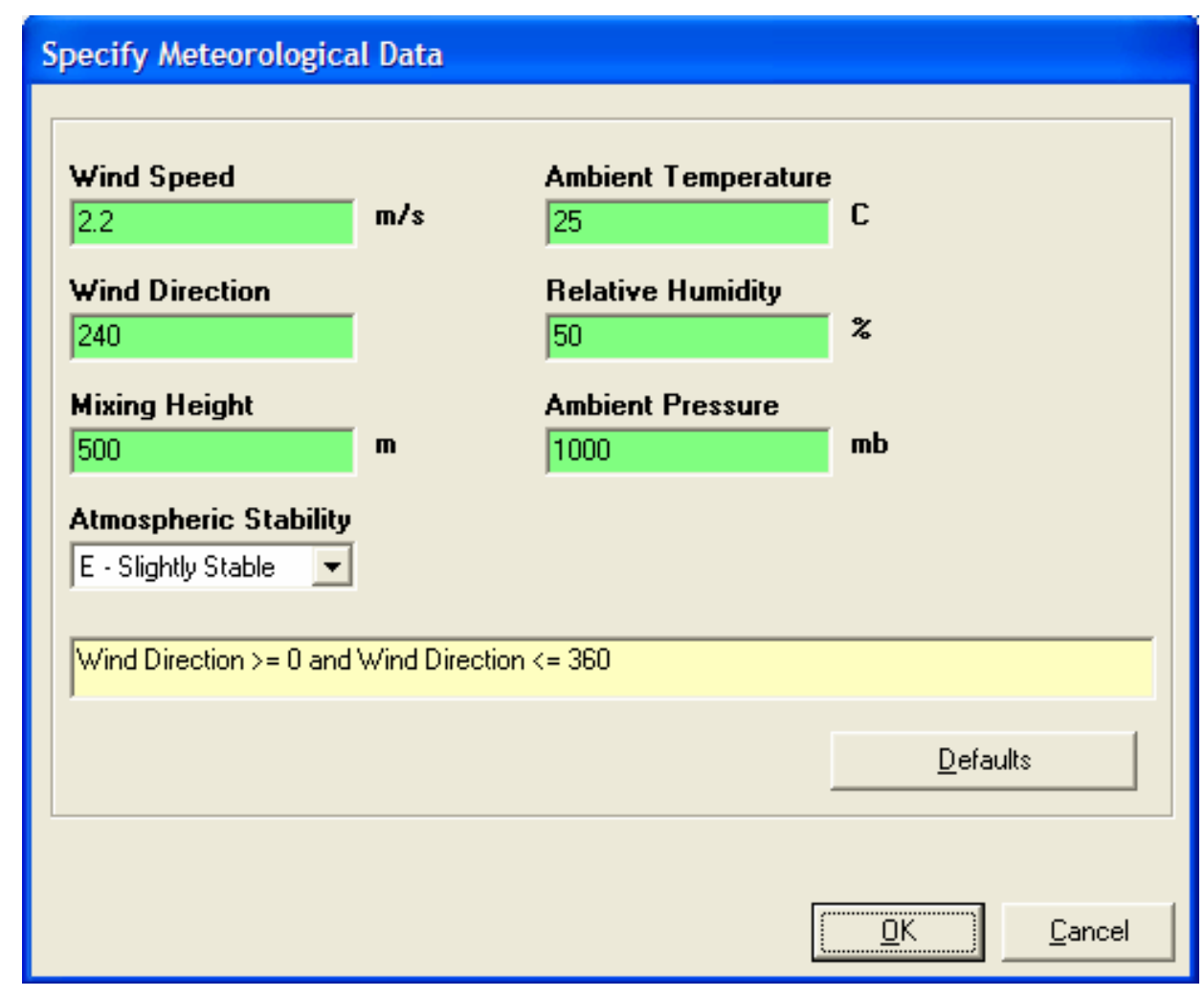

Figure 7.11. Completed Meteorological Form

\subsubsection{Setting Release and Simulation Duration}

The final step is to set the "Release Period" and "Simulation Scenario" details on the main DUSTRAN control panel (see Figure 7.12). The release duration is how long the sources release material, whereas the scenario duration is how long to simulate the released material. Set the Release Period's "Start Time" for 7 a.m. (early morning release) and set the "Release Duration" for 3 hrs. For the simulation, set the "Time Zone" to PST, the "Start Date" to June 16, 2005, the "Start Time" to 4 a.m., and the "Run Duration" to 8 hrs. (Note: the simulation start time must begin before sunrise, as CALMET, the meteorological model in DUSTRAN, needs to calculate the hourly surface heat flux from sunrise until the end of scenario or sunset, whichever occurs first.) Lastly, verify that the "Simulation Type" is set to "Source Emissions" as the scenario involves modeling the downwind dispersion from individual source types.

After entering the proper "Release Period" and "Simulation Scenario," click on the "Synchronize" button. Synchronization causes all the sources being simulated in DUSTRAN to have the same starting date, time, and duration. In this case, all sources will begin releasing material on June 16, 2005, at 7 a.m. PST and cease at 10 a.m. PST. The model will continue simulating the released material until 12 p.m., or until the concentration field no longer changes with time. 


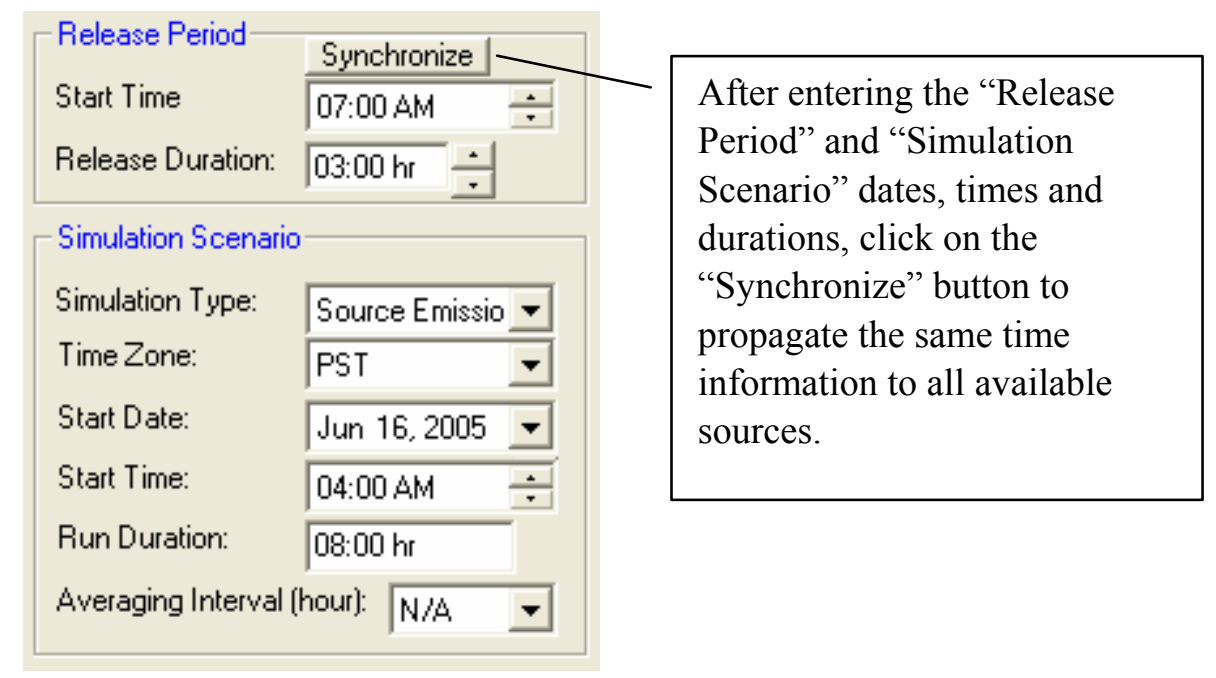

Figure 7.12. Portion of Main DUSTRAN Control Panel

\subsubsection{Running DUSTRAN}

After the sources, meteorology, and release duration information have been entered, a DUSTRAN simulation can be made. To run DUSTRAN, click on the "Run Simulation" button (see Figure 7.13).

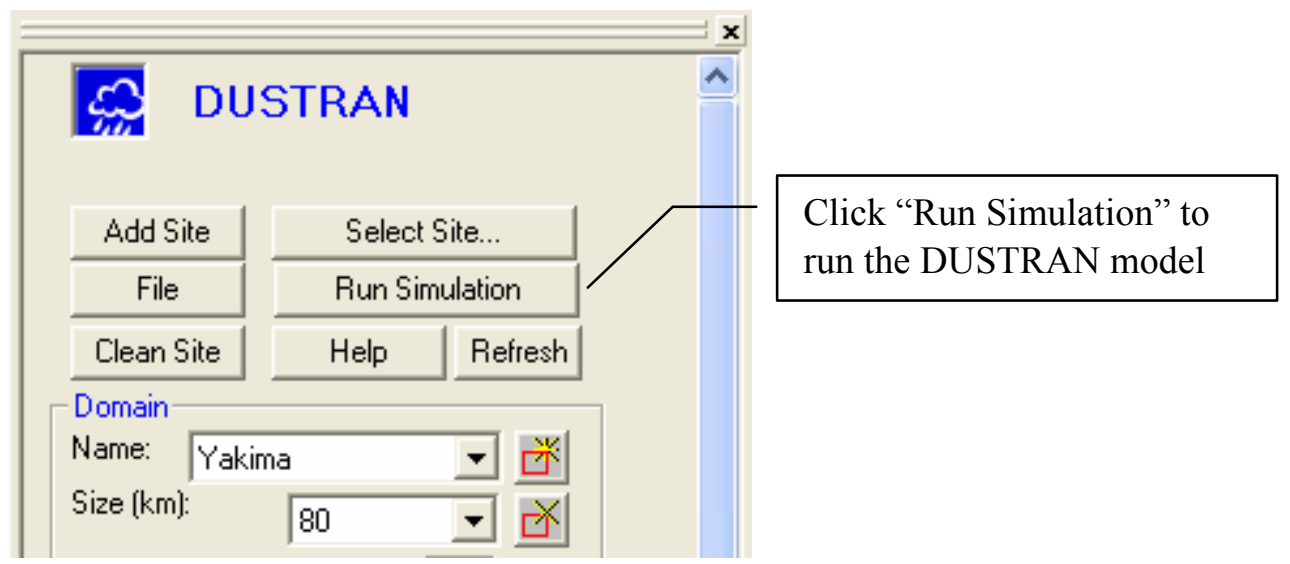

Figure 7.13. “Run Simulation” Button for Running DUSTRAN Simulation

\subsubsection{Displaying Model Output}

After the models finish running, click on the "Display Options" tab. Check "Contours" and "Wind Vectors" so that the plume contours and wind field will be displayed in ArcMap (see Figure 7.14). Wind vectors can be displayed for various layers throughout the domain; 20 meters is the top of the first layer, with mid-cell corresponding to 10 meters above ground level: 


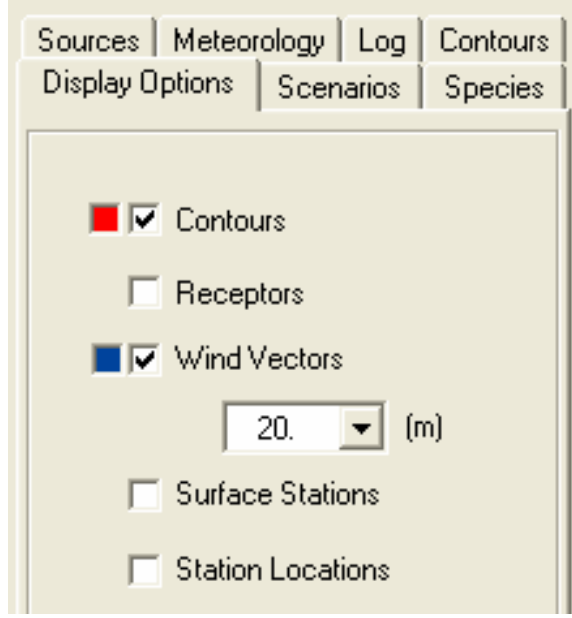

Figure 7.14. Choices After Clicking the "Display Options" Tab

\subsubsection{Viewing Model Results}

For each model time step, DUSTRAN calculates plume concentration and exposure as well as deposition and total deposition. To view a particular contour, click on the "Contours" tab and choose from the "Contour Types" listbox (see Figure 7.15):

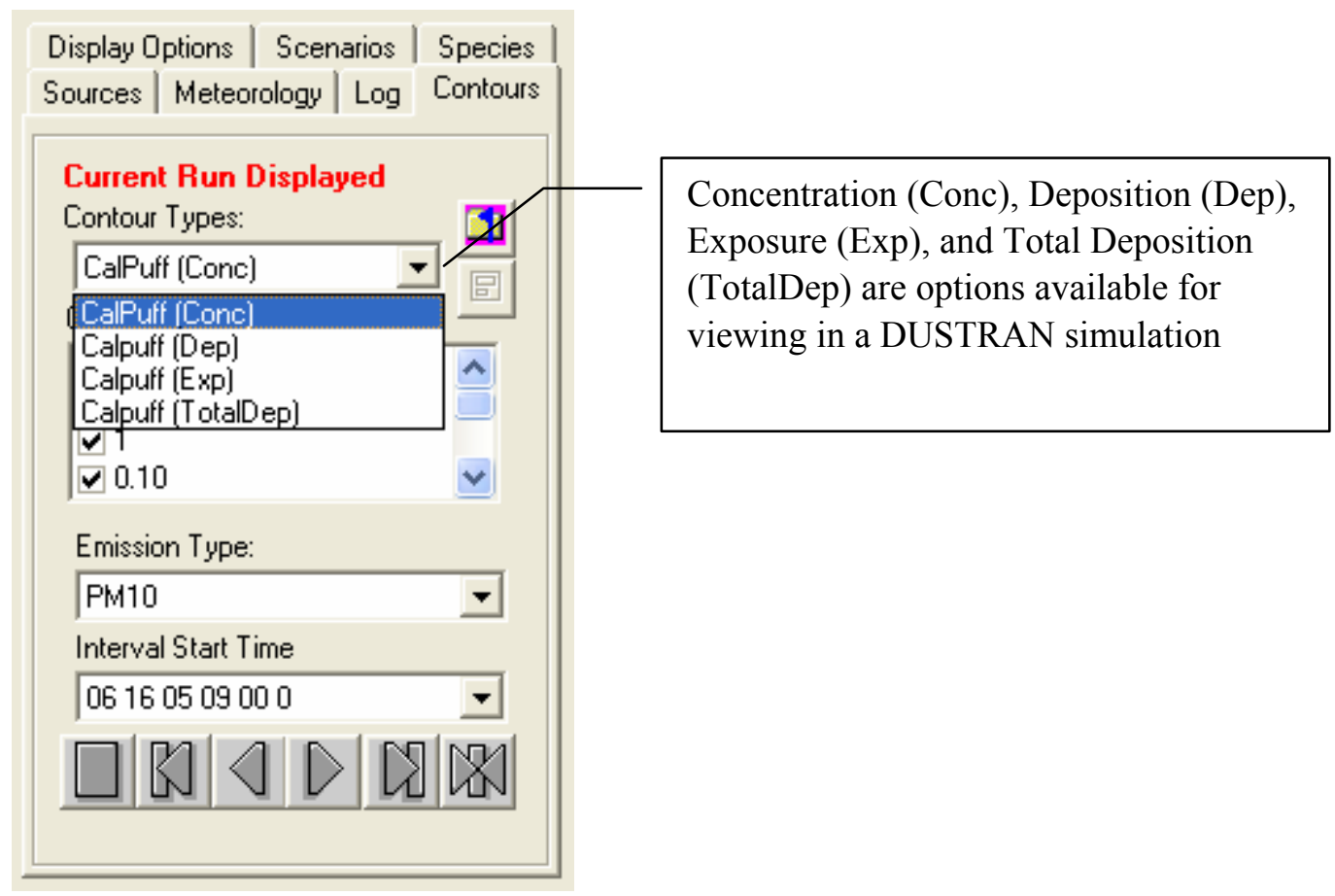

Figure 7.15. "Contour Types" Listbox

In this example, select "Conc" to display hourly concentrations within ArcMap. 
For a given contour type, numerous "Contours" are available for displaying. To display a particular contour interval, check the box next to the contour value. The default selection is normally adequate for displaying the maximum extent of the plume envelope.

To view a particular time step, select an interval from the "Interval Start Time" listbox. In this example, hourly time steps are available from the start of the release (7:00 a.m. local time) till the end of the run duration. Choose the 9:00 a.m. time step, which corresponds to the one hour (9:00 a.m. till 10:00 a.m.) average concentration (see Figure 7.16):

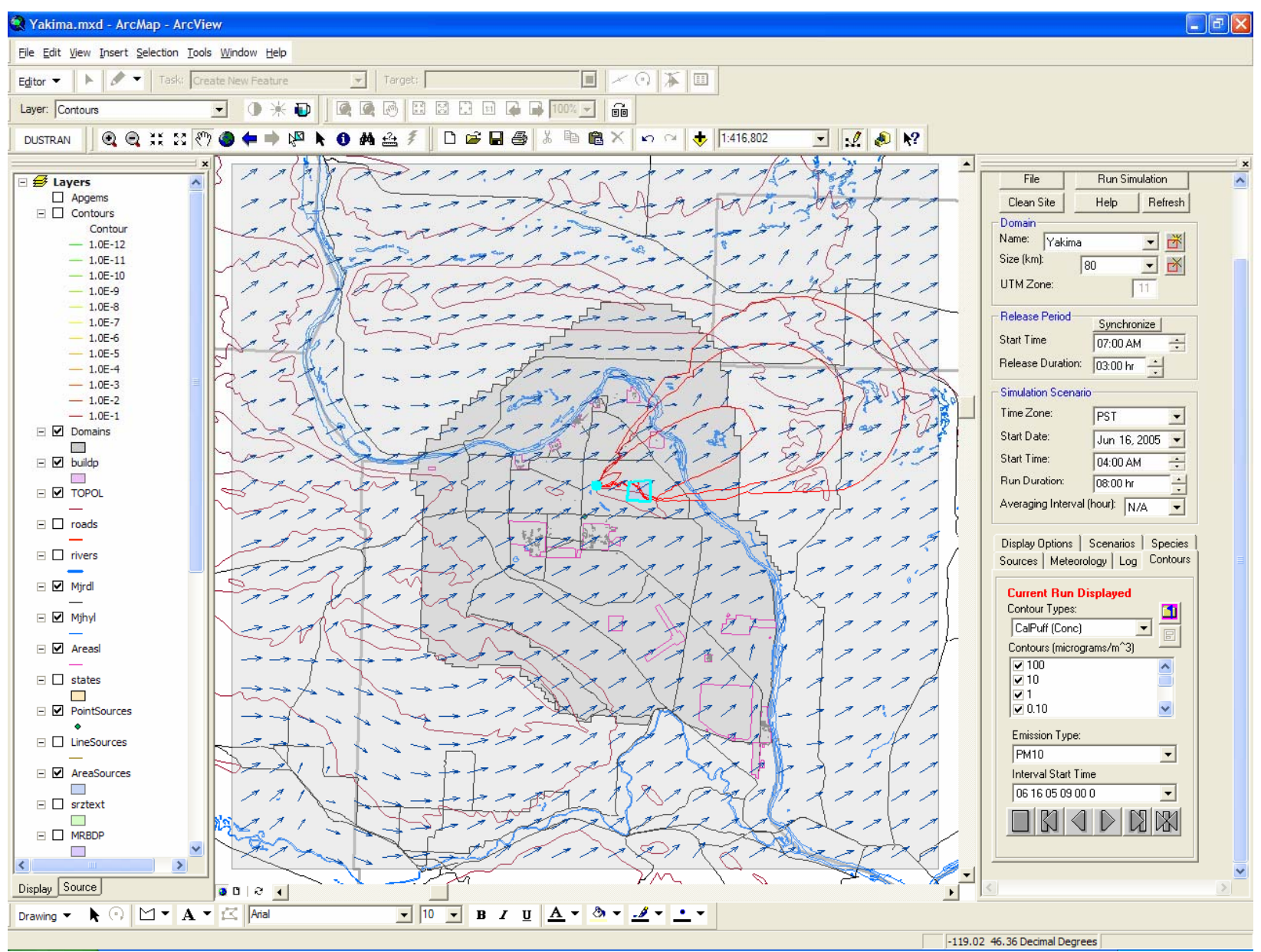

Figure 7.16. Display of Concentration Contours and Wind Vectors for the Hour from 9-10 AM

Notice in the above image that the two plumes (point and area-source plumes) have merged. In addition, note the distortion to the wind field due to the local topography. The distortion is caused by terrain blocking because of the early-morning stability and slope flow due to cold-air drainage.

\subsection{Simulating Wind-blown Dust Dispersion}

This tutorial steps through the process of simulating a wind-blown dust scenario. Gridded dust emissions are created automatically by the emissions module and are a function of wind speed, soil 
texture, and vegetation class. In this scenario, a high wind event is assumed to occur over a 3-hour period, and the resulting wind-blown dust transport and diffusion is simulated.

\subsubsection{Starting DUSTRAN}

DUSTRAN is an integrated dispersion modeling application within the ArcMap (Version 9.x) GIS interface. To begin a DUSTRAN simulation, open ArcMap. On the ArcMap toolbar (see Figure 7.17), click on the "DUSTRAN" button:

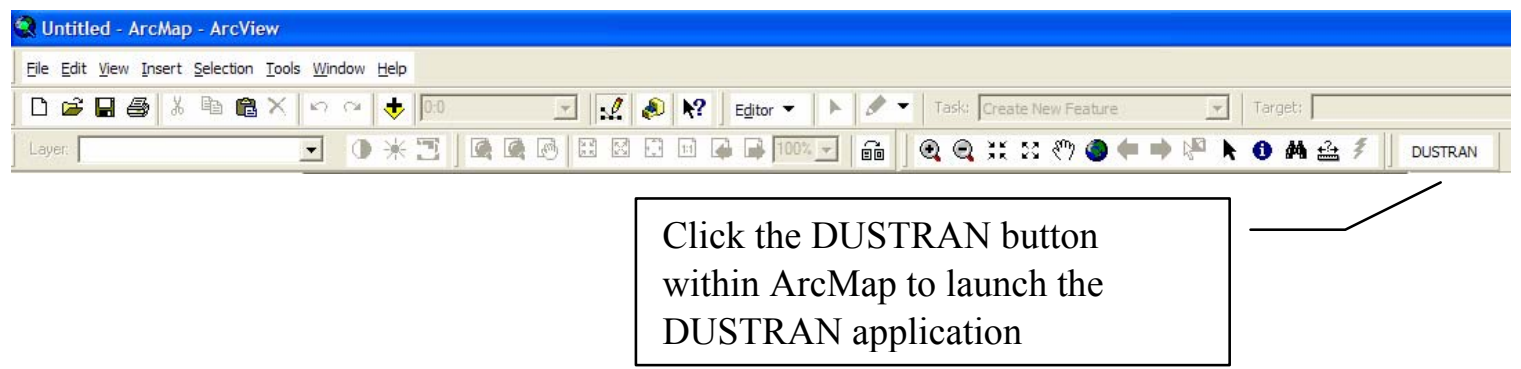

Figure 7.17. ArcMap Toolbar

\subsubsection{Selecting a Site}

The user interface to the DUSTRAN model will appear in a frame within the ArcMap application (see Figure 7.18). Click the "Select Site..." button to open a dialog box which allows the user to select an existing site:

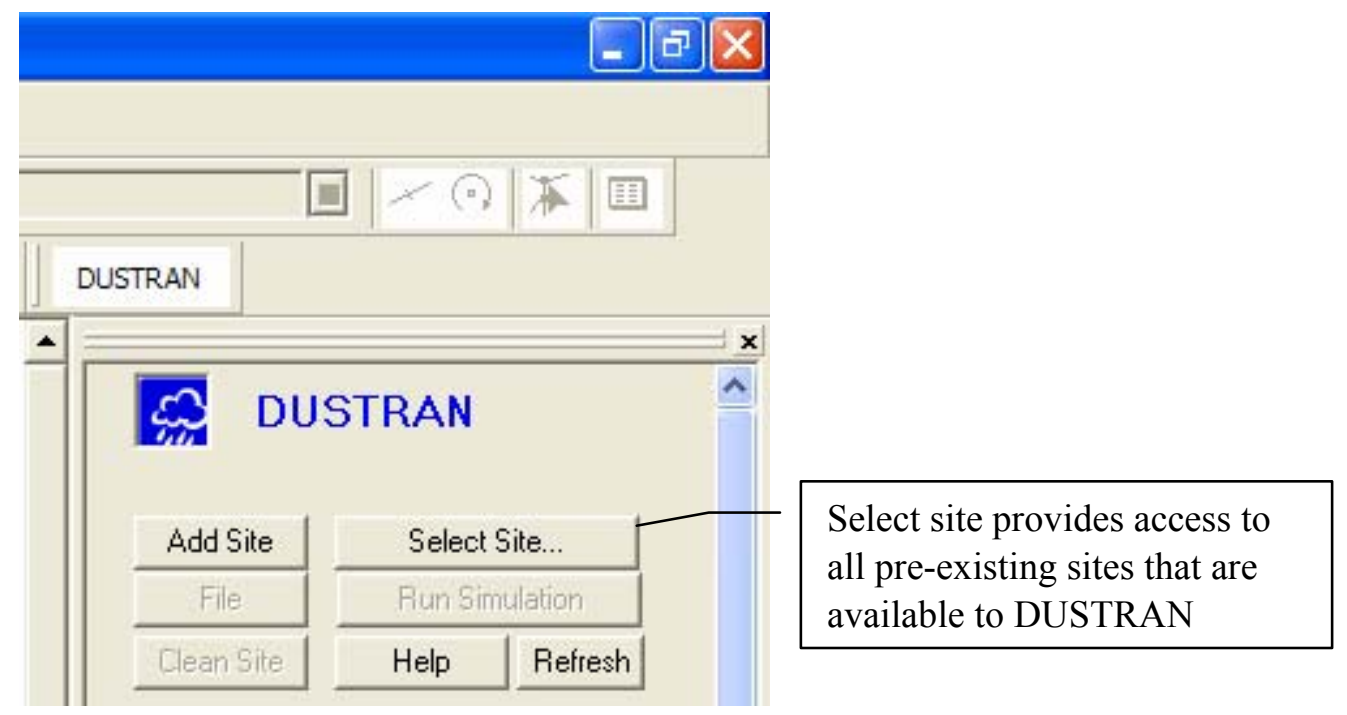

Figure 7.18. Portion of User Interface to the DUSTRAN Model

Select "Yakima" from the list of available model (see Figure 7.19) sites and then click "Open": 


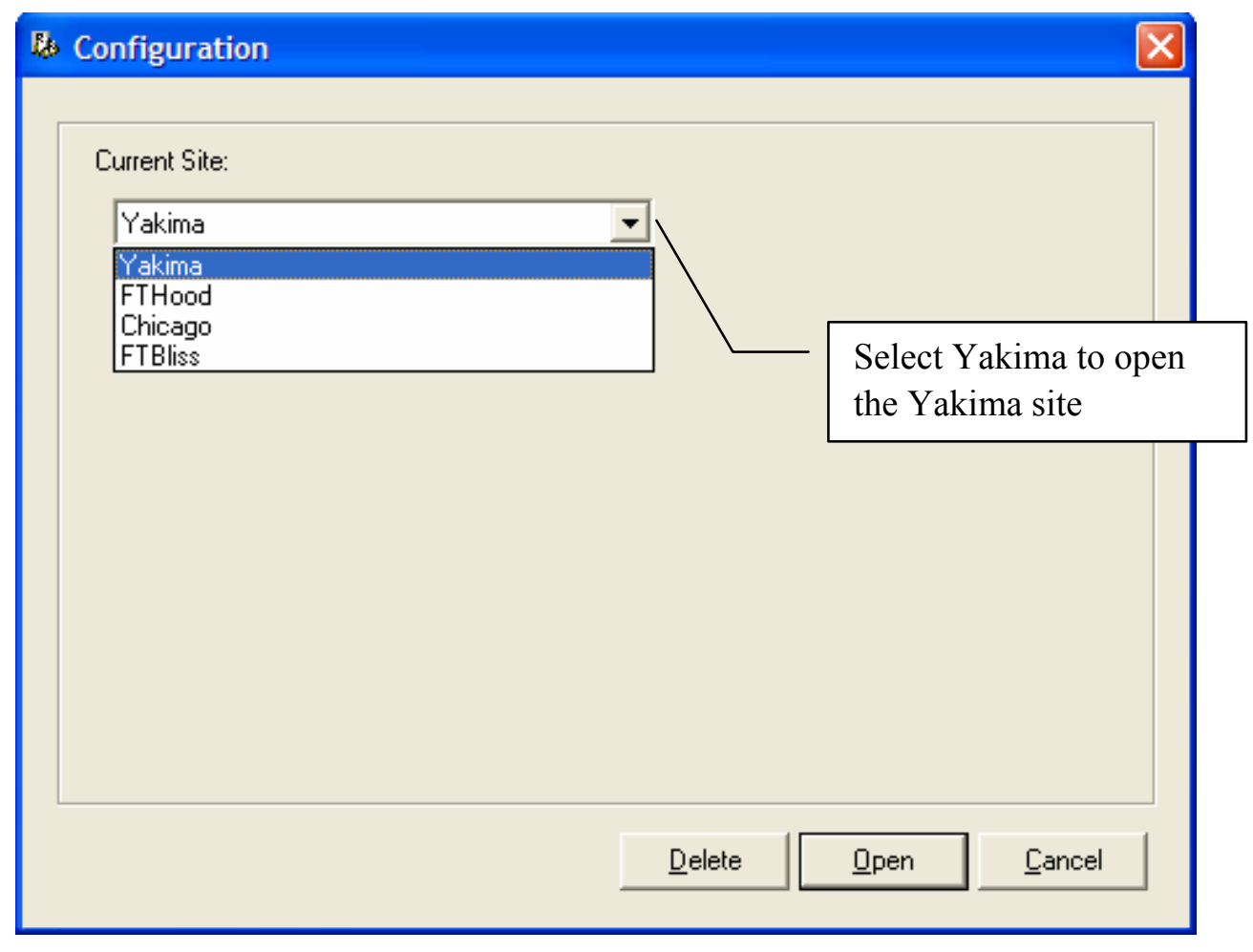

Figure 7.19. List of Available Model Sites

The Yakima site will open within ArcMap. A list of available GIS data layers will appear in the left frame and DUSTRAN-specific input parameters will appear in the right frame (see Figure 7.20): 


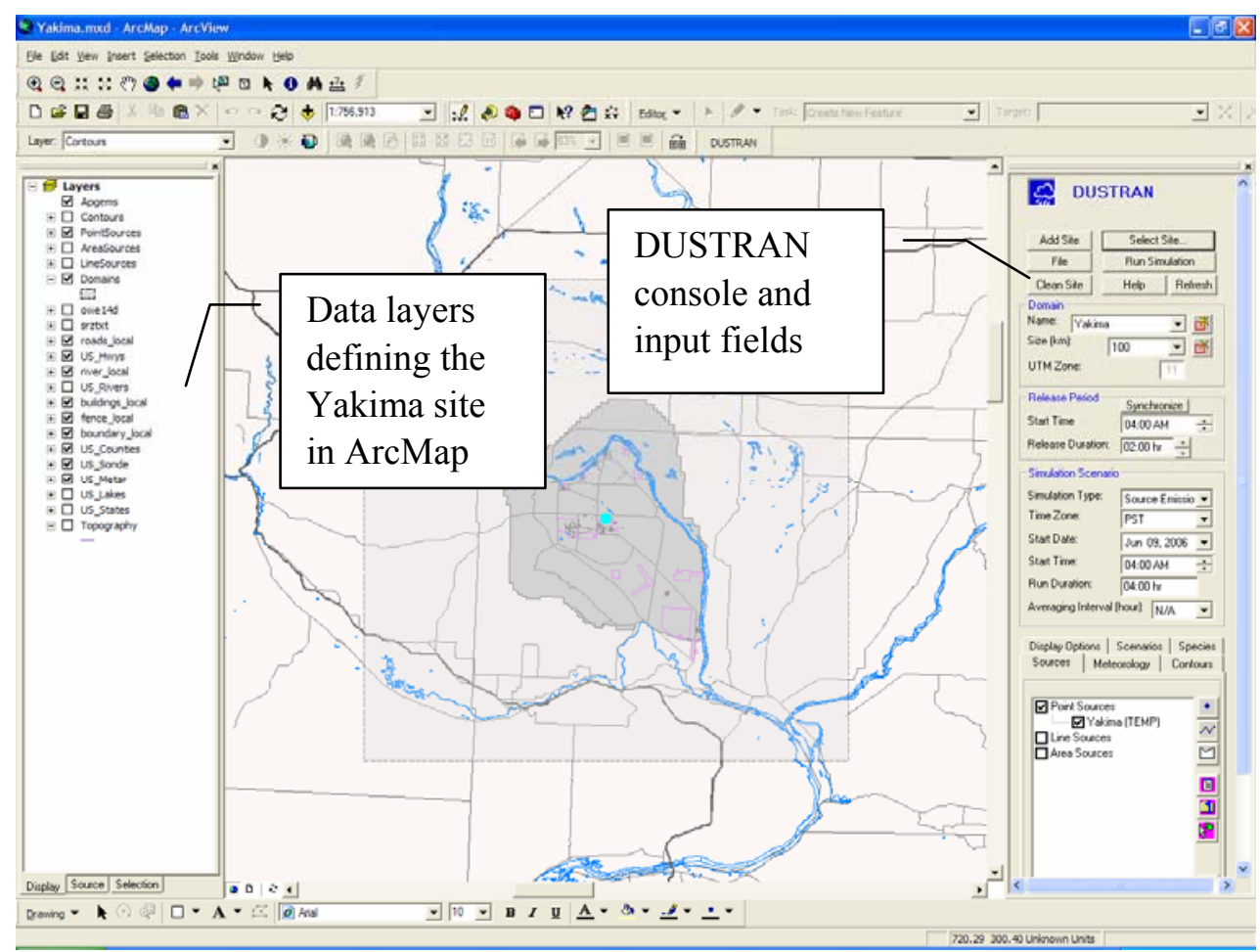

Figure 7.20. Display of Yakima Site

\subsubsection{Defining the Domain}

In a wind-blown dust simulation, the domain represents the area from which dust emissions will be generated. In addition, the domain is the area where both meteorological and dispersion calculations are performed.

In this example, an existing domain, called "Yakima," will be used. From the "Domain" panel within DUSTRAN, select the domain "Named" Yakima and set the domain "Size" to $200 \mathrm{~km}$. The Yakima site should appear as follows within ArcMap (see Figure 7.21): 


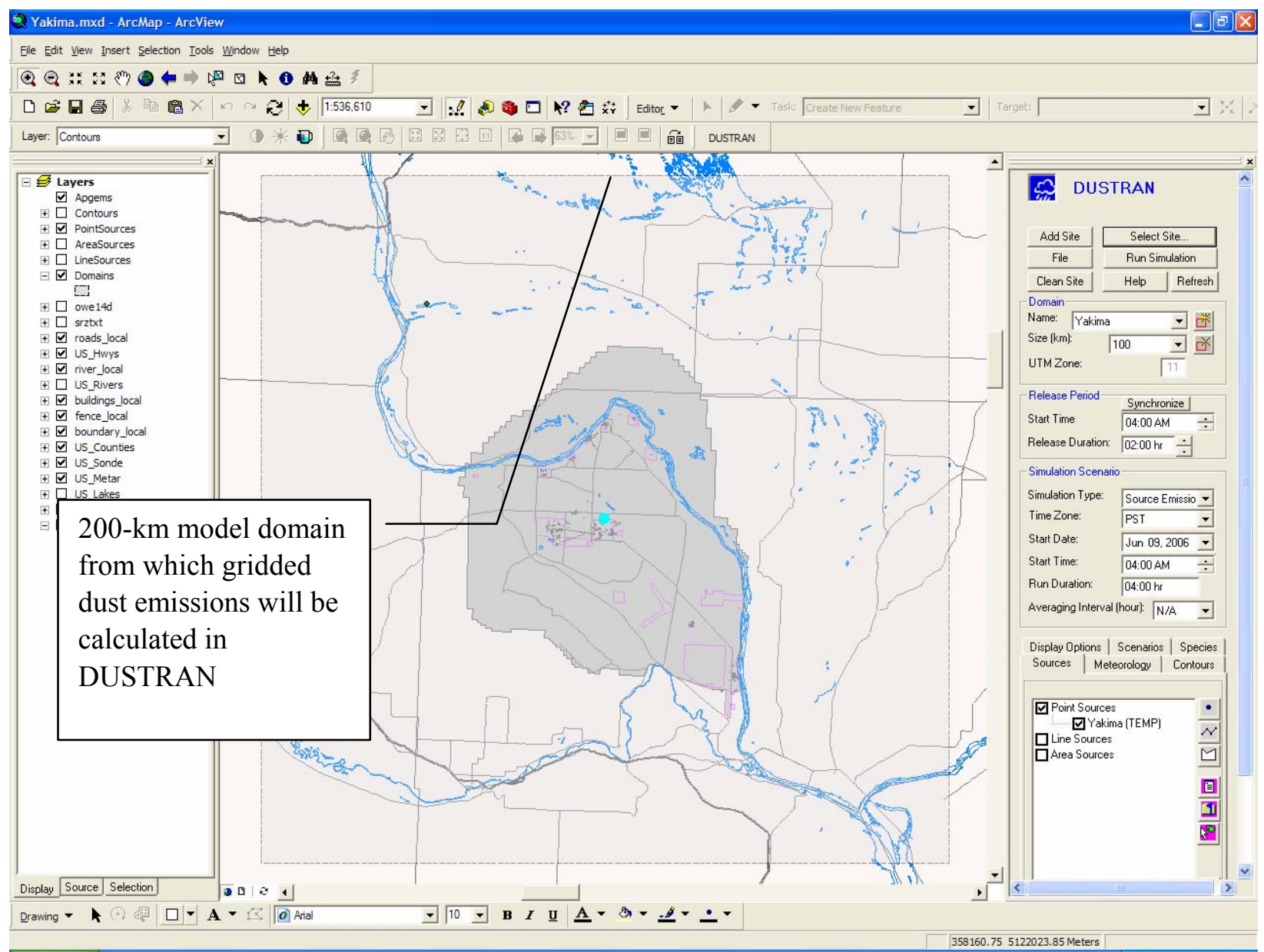

Figure 7.21. Display of 200-km-Square Yakima Domain Within Yakima Site

\subsubsection{Setting the Simulation Scenario}

This scenario will simulate wind-blown dust during a 3-hour wind event that occurs in the early morning hours of April 15, 2005. In the "Simulation Scenario" panel, set the "Simulation Type" to "Wind-blown Dust" and the "Time Zone," "Start Date," "Start Time," and "Run Duration" as shown in Figure 7.22:

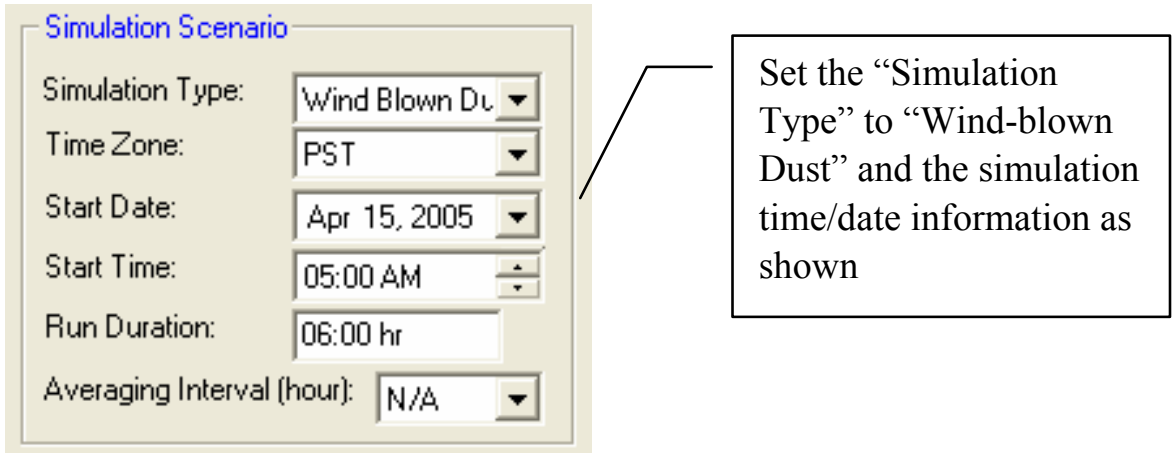

Figure 7.22. "Simulation Scenario" Panel 


\subsubsection{Setting the Soil and Vegetation Characteristic Files}

Zobler soil texture and Olson Ecosystem vegetation class files are generated whenever a site is created using the "Add Site" wizard within the DUSTRAN interface; these files are required to calculate dust emissions for the domain. In addition, the "Polygon Layer Creator" utility can be used to create high-resolution characteristic files for direct use in DUSTRAN (see Polygon Layer Creator Utility, Section 5.2).

In this example, the characteristic files that are generated with the site will be used. To add soil and vegetation files, select the "Sources" tab and click on the "Characteristic Files" button (see Figure 7.23):

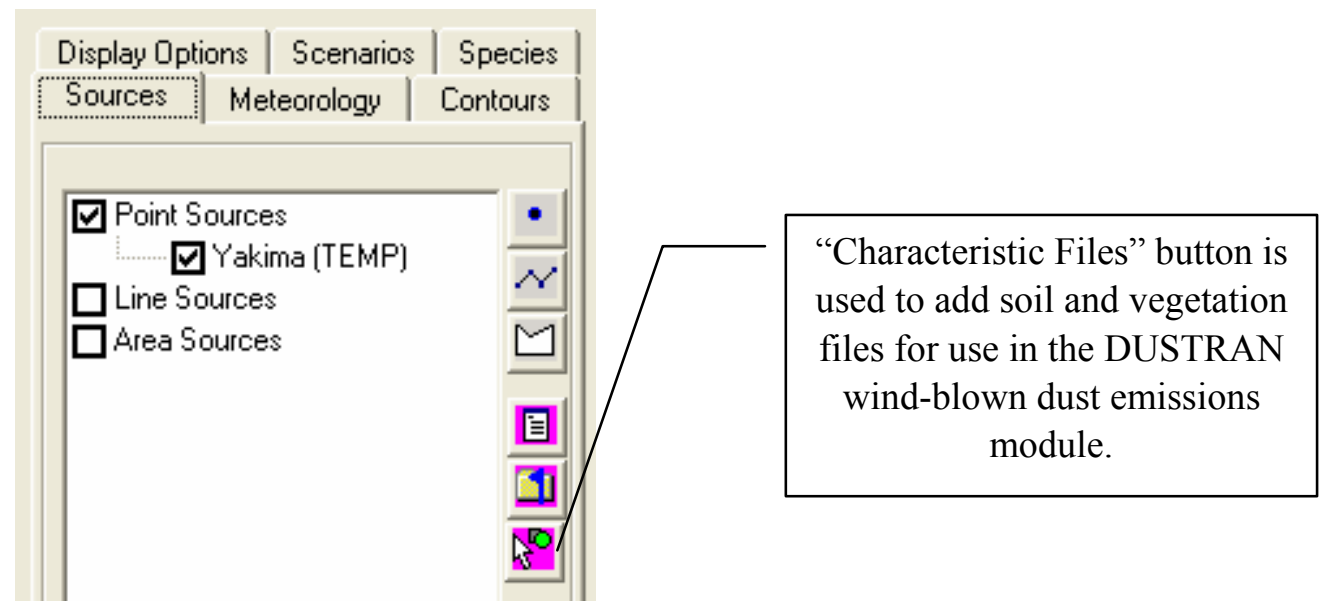

Figure 7.23. Adding Soil and Vegetation Files from DUSTRAN User Interface

After clicking the "Characteristics File" button, a form will appear that allows for the selection of both the "Soils" and "Vegetation" categories (see Figure 7.24):

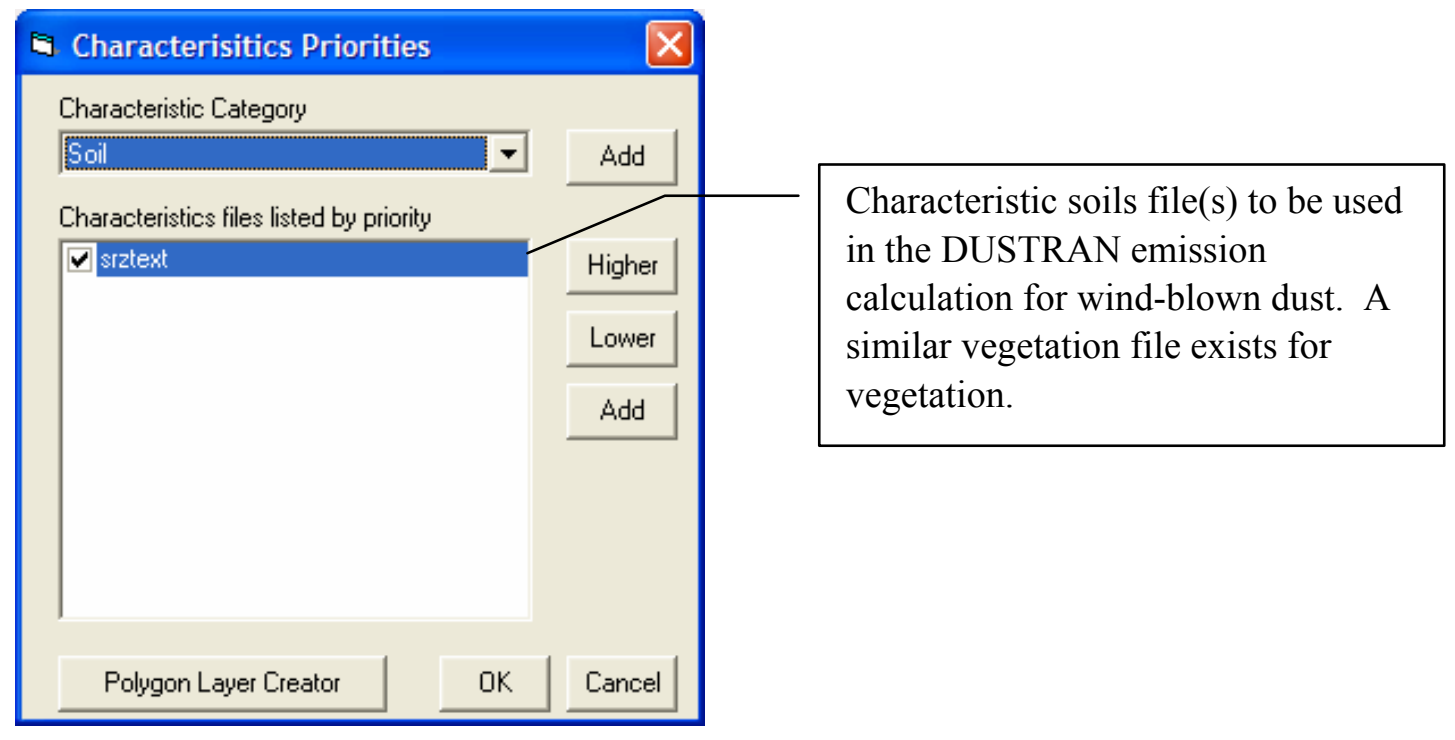

Figure 7.24. “Characteristics Priorities" Form 
The standard soils file created for the site is called "srztext" and is selected by default. The file contains gridded values of Zobler soil textures for the site. Similarly, an Olson World Ecosystem gridded file, called "owe14d," exists for the vegetation category and is selected by default. Site-specific soils and vegetation files can be added for use in the emissions calculations; however, they must use the Zobler or Olson identification system (see Windblown Dust, Section 2.5.2 for more information on these classifications).

\subsubsection{Viewing the Soil and Vegetation Characteristic Files}

Shape files, which represent the various soil and vegetation categories, can be displayed within ArcMap so that potential dust-emission regions within the domain can be readily identified. Default shape files are automatically created with the site and stored in the "TerData" directory for the site. These files can be added to ArcMap as "Layers" for display.

These Zobler soil and Olson World Ecosystem shape files have already been added to the Yakima site. The layers are turned off by default, but can be activated by "checking" the box next to each layer's respective name.

To turn on the soil's layer, "check" the layer called "srztxt" (see Figure 7.25). Similarly, to turn on the vegetation layer, "check" the layer called "owe14d":

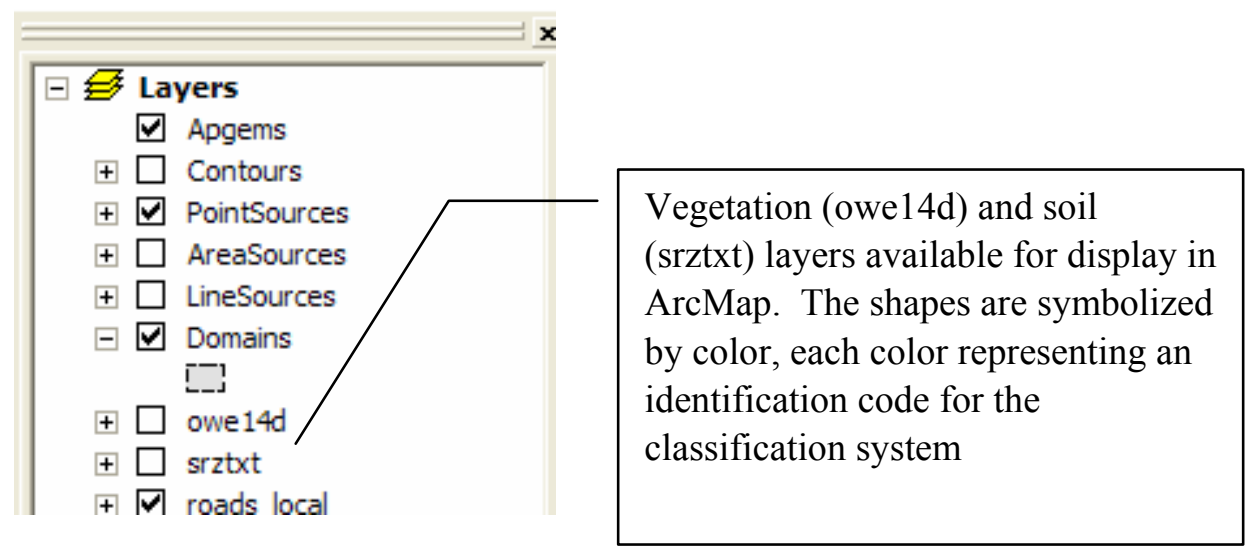

Figure 7.25. Display Showing Choices for Soil and Vegetation Layers

Figure 7.26 displays the Olson vegetation layer for the Yakima site. Each colored polygon represents an Olson World Ecosystem category ID, the values of which are displayed when the "Layer" is expanded. Of all the vegetation codes available, only four have been identified as allowing for effective wind-blown dust emissions. These categories, discussed in Section 2.5.2.4, include 8, 50, 51, and 52. For the Yakima site, only category 52 - Cool/cold shrub, semi-desert/steppe exists within the domain; these are the red polygon regions and are the only potential source locations for wind-blown dust. 


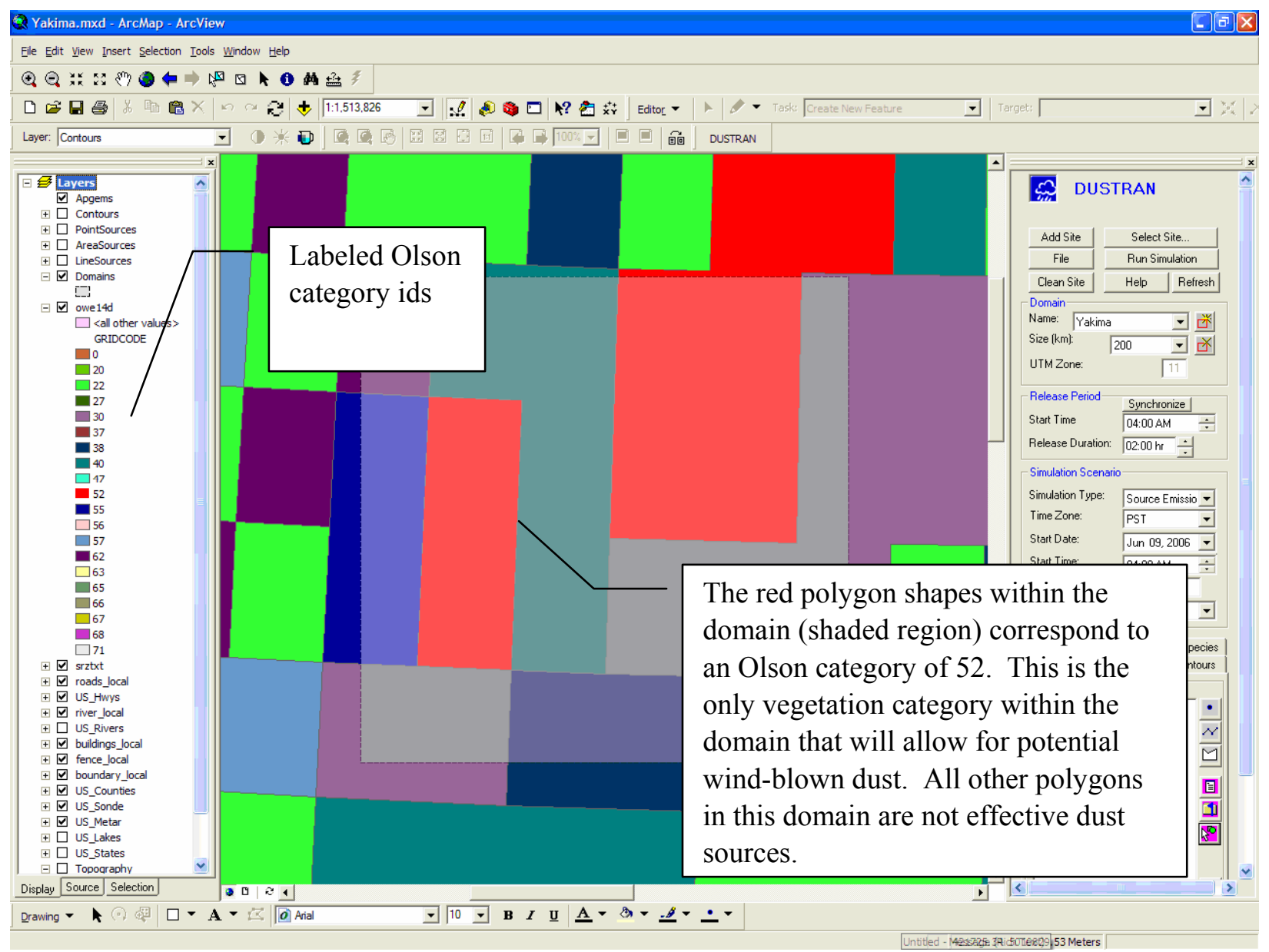

Figure 7.26. Display of Olson Vegetation Layer for the Yakima Site

\subsubsection{Entering Meteorological Data}

The final step before running the simulation is to set the meteorology. Click on the "Meteorology" tab within DUSTRAN and select "Single Observation" from the listbox. The "Specify Meteorological Data" form appears. Enter the meteorological parameters as shown in the form below. Select the "Atmospheric Stability" as "D - Neutral," a wind speed of $10 \mathrm{~m} / \mathrm{s}$, and a wind direction from the southwest (240 degrees). The completed form will appear as shown in Figure 7.27: 


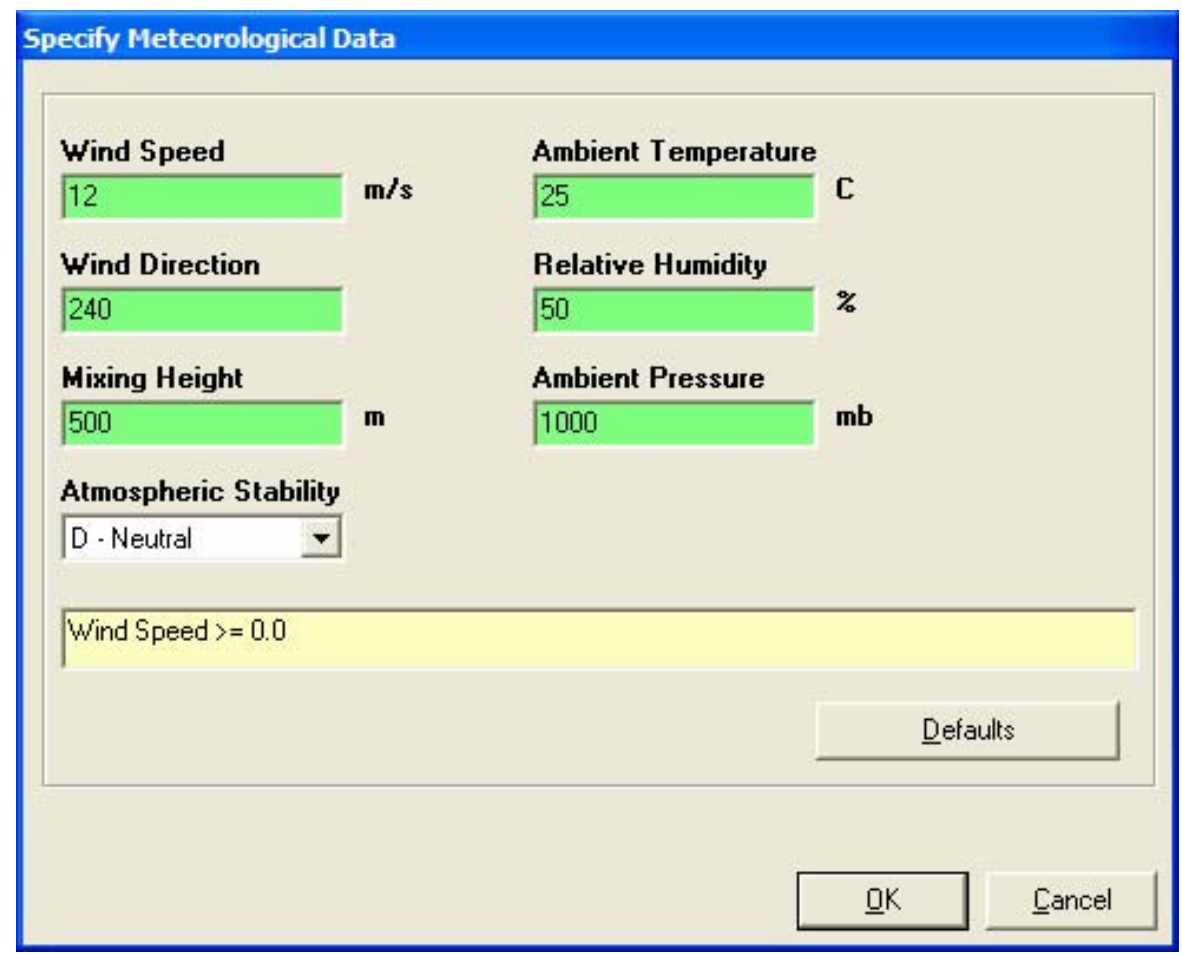

Figure 7.27. Completed "Specify Meteorological Data” Form

\subsubsection{Running DUSTRAN}

After the domain, simulation details, soil/vegetation categories, and meteorology have been entered, a wind-blown dust simulation in DUSTRAN can be made. To run DUSTRAN, click on the "Run Simulation" button (see Figure 7.28).

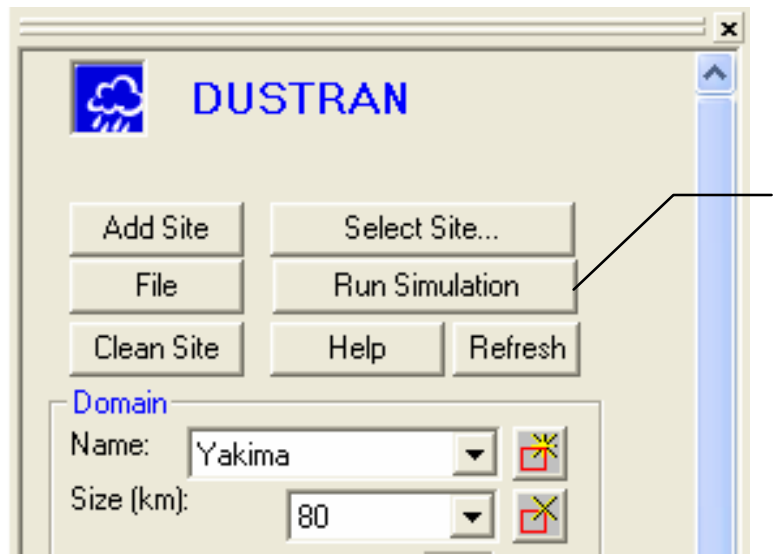

Click "Run Simulation" to run the DUSTRAN model

Figure 7.28. “Run Simulation” Button in DUSTRAN User Interface 


\subsubsection{Viewing Model Results}

For each model time step, DUSTRAN calculates plume concentration and exposure as well as deposition and total deposition. To view a particular contour, click on the "Contours" tab and choose from the "Contour Types" listbox (see Figure 7.29):

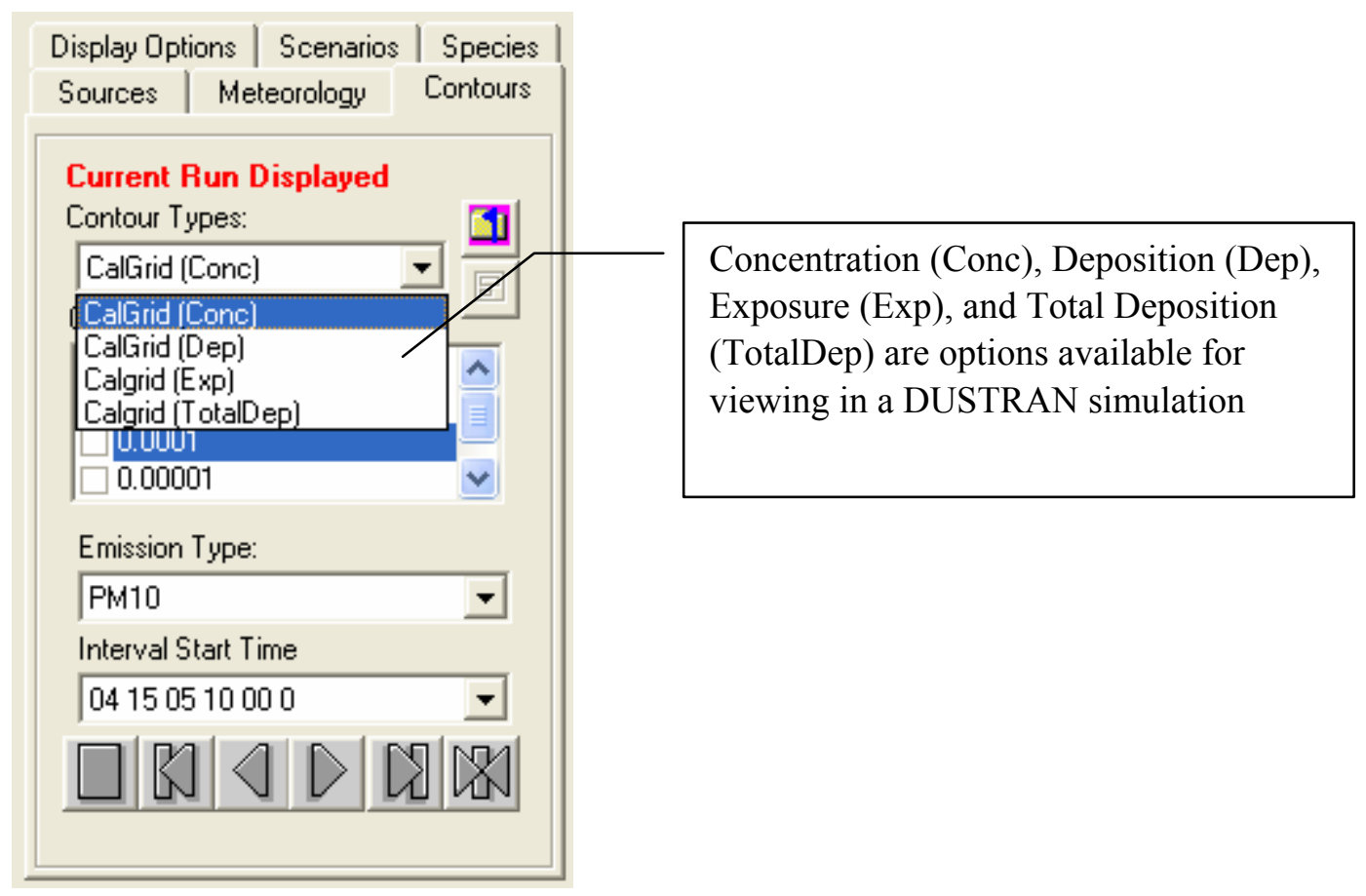

Figure 7.29. “Contours Types” Listbox

In this example, select "Conc" to display hourly concentrations within ArcMap.

For a given contour type, numerous "Contours" are available for display. To display a particular contour interval, check the box next to the contour value. The default selection is normally adequate for displaying the maximum extent of the plume envelope.

To view a particular time step, select an interval from the "Interval Start Time" listbox (see Figure 7.30). In this example, hourly time steps are available from the start of the release (5:00 a.m. local time) till the end of the simulation. Choose the 7:00 a.m. time step, which corresponds to the one hour (7:00 a.m. till 8:00 a.m.) average concentration: 


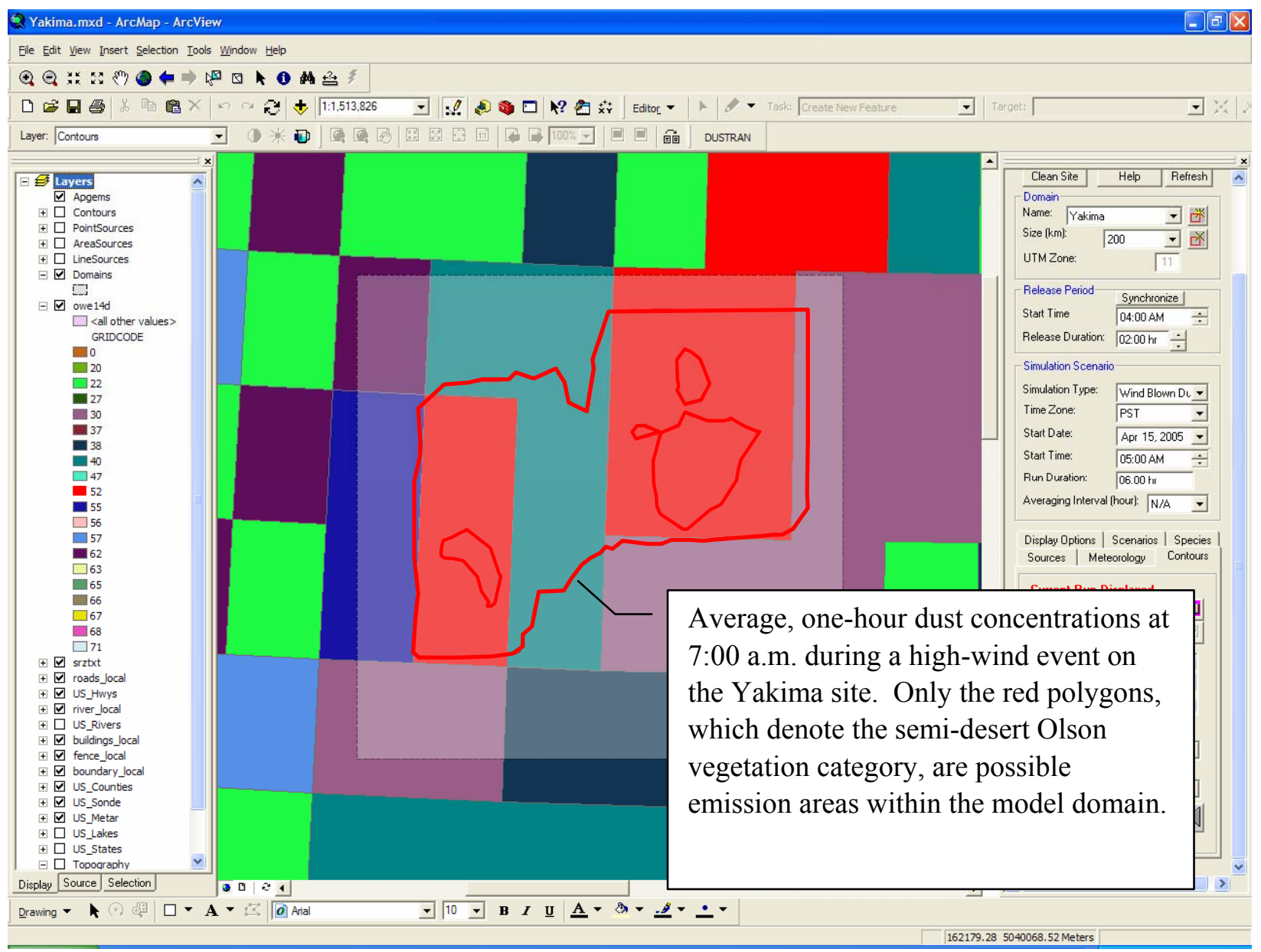

Figure 7.30. Display of Concentration Contours for the Hour from 7-8 AM

In the above image, the Olson vegetation coverage (colored polygons) and plume contours are displayed. Only the red polygons (Olson category 52-Cool/cold shrub, semi-desert/steppe) are potential source regions (as discussed previously) and are the only regions within the domain with plume contours. 


\subsection{References}

Allwine KJ, FC Rutz, JP Rishel, JG Droppo, SL Bird, and H Thistle. 2006. SPRAYTRAN 1.0 User's Guide: A GIS-Based Atmospheric Spray Droplet Dispersion Model. PNNL-16075, Pacific Northwest National Laboratory, Richland, WA.

Fecan F, B Marticorena, and G Bergametti. 1999. "Parameterization of the increase of the Aeolian erosion threshold wind friction velocity due to soil moisture for arid and semi-arid areas.” Annales Geophys. 17:194-157.

Gillette DA, and R Passi. 1988. “Modeling dust emission caused by wind erosion.” J. Geophys. Res. 93:14233-14242.

Gillies JA, V Etyemezian, H Kuhns, D Nikolic, and DA Gillette. 2005a. "Effect of vehicle characteristics on unpaved road dust emissions.” Atmos. Environ. 39:2341-2347.

Gillies JA, WP Arnott, V Etyemezian, H Kuhns, H Moosmuller, D DuBois, M Abu-Allaban, G Schwemmer, DA Gillette, WG Nickling, R Varma, T Wilkerson, and R Varma. 2005b. Characterizing and Quantifying Local and Regional Particulate Matter Emissions from Department of Defense Installations. Final report prepared for the Strategic Environmental Research and Development Program under Project CP-1191, March 2005.

Irwin JS. 1998. Interagency Workgroup on Air Quality Modeling (IWAQM) Phase 2 Summary Report and Recommendations for Modeling Long Range Transport Impacts. EPA-454/R-98-019, Office of Air Quality Planning and Standards, Research Triangle Park, NC, 151 pp. (NTIS Accession Number PB 99-121089).

Nickovic S, G Kallos, A Papadopoulos, and O Kakaliagou. 2001. “A model for prediction of desert dust cycle in the atmosphere.” J. Geophys. Res. 106, 18113-18129.

Olson JS. 1992. "World Ecosystems (WE1.4). Digital Raster Data on a 10-minute Cartesian Orthonormal Geodetic $1080 \times 2160$ grid.” In: Global Ecosystems Database, Version 2.0. Boulder, CO. Available at: http://www.ngdc.noaa.gov/seg/cdroms/ged iia/datasets/a05/ow.htm (accessed 08-15-2006).

Scire JS, DG Strimaitis, and RJ Yamartino. 2000a. A User's Guide for the CALPUFF Dispersion Model (Version 5). Earth Tech, Inc., Concord, MA.

Scire JS, FR Robe, ME Fernau, and RJ Yamartino. 2000b. A User's Guide for the CALMET Meteorological Model (Version 5). Earth Tech, Inc., Concord, MA.

Scire JS, RJ Yamartino, GR Carmichael, and YS Chang. 1989. CALGRID: A Mesoscale Photochemical Grid Model Volume II - User's Guide. California Resources Board, Sacramento, CA.

Staub B, and C Rosenzweig. 1992. "Global Zobler Soil Type, Soil Texture, Surface Slope, and Other Properties. Digital Raster Data on a 1-degree Geographic (lat/long) $180 \times 360$ grid.” In: Global 
Ecosystems Database Version 2.0. Boulder, CO, NOAA National Geophysical Data Center. Available at: http://www.ngdc.noaa.gov/seg/cdroms/ged_iia/datasets/a11/sr.htm (accessed 08-15-2006).

Tegen I, and I Fung. 1994. "Modeling of mineral dust in the atmosphere: sources, transport, and optical thickness." J. Geophys. Res. 99:22987-22914.

Turner DB. 1994. Workbook of Atmospheric Dispersion Estimates: An Introduction to Dispersion Modeling. Second Edition. ISBN 1-56670-023-X. CRC Press, Inc. Boca Raton, FL, 33431.

U.S. Environmental Protection Agency (EPA). 2005. "Compilation of Air Pollutant Emission Factors (AP-42) Fifth Edition, Volume 1: Stationary Point and Area Sources." U. S. Environmental Protection Agency, Research Triangle Park, NC. Available at http://www.epa.gov/ttn/chief/ap42/ (accessed 08-15-2006). 
Appendix A

\section{DUSTRAN Directory and File Documentation}




\section{Appendix A: DUSTRAN Directory and File Documentation}

This appendix provides detailed information about the DUSTRAN directory structure and the supporting files used within the modeling system. The section begins with an overview of the folders and files that are within the root DUSTRAN directory. Input files that are stored within each site's "StaticData" directory are then defined. These files may be edited by an advanced user who wishes to control certain options not available within the DUSTRAN interface or control the behavior of the CALMET, CALPUFF, and CALGRID models. An overview of DUSTRAN's geodatabase is then given and is provided as a reference for users who wish to interact with the model data within ArcMap. Lastly, detailed information about the structure of the dust emission input and output files (Ldustinp.txt and Ldustout.txt) are provided.

\section{A.1 DUSTRAN Directory}

The DUSTRAN directory is the main directory used by the modeling system. This directory is normally a root directory on the machine's primary hard drive (e.g., c: IDUSTRAN). The main directory contains the executables used by the modeling system as well as the dynamic link library (DUSTRAN.dll) that integrates the modeling system with the ArcMap application. This directory also contains the individual site directories that are used within DUSTRAN simulations. The following table outlines the directories and files that are present in the DUSTRAN directory.

\begin{tabular}{|l|l|}
\hline Name & Description \\
\hline TerrFiles & $\begin{array}{l}\text { Directory in which the land-use and terrain files used in the simulations are } \\
\text { stored. }\end{array}$ \\
\hline ModelFiles & $\begin{array}{l}\text { Template model and processor input files are stored in this directory. The } \\
\text { Add Site wizard uses these templates during the creation of a new site. }\end{array}$ \\
\hline Site Directories & $\begin{array}{l}\text { For each site present in the modeling system, a directory with the name of the } \\
\text { site will be present. This directory is used to store all data that are specific to } \\
\text { the site. }\end{array}$ \\
\hline Areadust.exe & Wind-blown dust emission model executable. \\
\hline CalConc.exe & $\begin{array}{l}\text { Executable that processes the output of the CALPUFF and CALGRID } \\
\text { models into the contours that are displayed in the map display of ArcMap. }\end{array}$ \\
\hline Calgrid.exe & CALGRID dispersion model executable. \\
\hline Calmet.exe & CALMET meteorological model executable. \\
\hline Calpuff.exe & CALPUFF dispersion model executable. \\
\hline Ctgproc.exe & Ctgproc land-use file processor executable. \\
\hline LineDust.exe & Line and area dust-emission model executable. \\
\hline Makegeo.exe & Makegeo land-use and terrain file processor executable. \\
\hline Read62.exe & $\begin{array}{l}\text { Upper air station meteorological data processor executable. This processor } \\
\text { works with the Forecast System Laboratory (FSL) file format taken from the } \\
\text { NOAA website. }\end{array}$ \\
\hline Readmeviewer.exe & $\begin{array}{l}\text { Executable that displays the readme.txt file before the first startup of } \\
\text { DUSTRAN. }\end{array}$ \\
\hline Terrel.exe & Terrain file processor executable. \\
\hline
\end{tabular}




\begin{tabular}{|c|c|}
\hline Name & Description \\
\hline Dustran.dll & DUSTRAN dynamic link library. \\
\hline Calmet.inp & $\begin{array}{l}\text { Input file used by the CALMET model. This file is generated by the } \\
\text { modeling system before the execution of the model. }\end{array}$ \\
\hline Calpuff.inp & $\begin{array}{l}\text { Input file used by the CALPUFF model. This file is generated by the } \\
\text { modeling system before the execution of the model. }\end{array}$ \\
\hline Calgrid.inp & $\begin{array}{l}\text { Input file used by the CALGRID model. This file is generated by the } \\
\text { modeling system before the execution of the model. }\end{array}$ \\
\hline Ctgproc.inp & $\begin{array}{l}\text { Input file used by the Ctgproc processor. This file is generated by the } \\
\text { modeling system before the execution of the processor. }\end{array}$ \\
\hline Makegeo.inp & $\begin{array}{l}\text { Input file used by the Makegeo processor. This file is generated by the } \\
\text { modeling system before the execution of the processor. }\end{array}$ \\
\hline Read62.inp & $\begin{array}{l}\text { Input file used by the Read } 62 \text { processor. This file is generated by the } \\
\text { modeling system before the execution of the processor. }\end{array}$ \\
\hline Terrel.inp & $\begin{array}{l}\text { Input file used by the Terrel processor. This file is generated by the } \\
\text { modeling system before the execution of the processor. }\end{array}$ \\
\hline Calmet.lst & $\begin{array}{l}\text { Output file generated by the CALMET model, which details the success or } \\
\text { failure of the model run. }\end{array}$ \\
\hline Calpuff.lst & $\begin{array}{l}\text { Output file generated by the CALPUFF model, which details the success or } \\
\text { failure of the model run. }\end{array}$ \\
\hline Calgrid.lst & $\begin{array}{l}\text { Output file generated by the CALGRID model, which details the success or } \\
\text { failure of the model run. }\end{array}$ \\
\hline Ctgproc.lst & $\begin{array}{l}\text { Output file generated by the Ctgproc processor, which details the success or } \\
\text { failure of the processor run. }\end{array}$ \\
\hline Makegeo.lst & $\begin{array}{l}\text { Output file generated by the Makegeo processor, which details the success or } \\
\text { failure of the processor run. }\end{array}$ \\
\hline Read62.1st & $\begin{array}{l}\text { Output file generated by the Read } 62 \text { processor, which details the success or } \\
\text { failure of the processor run. }\end{array}$ \\
\hline Terrel.lst & $\begin{array}{l}\text { Output file generated by the Terrel processor, which details the success or } \\
\text { failure of the processor run. }\end{array}$ \\
\hline Convert.csv & $\begin{array}{l}\text { Comma-separated text file containing the unit conversion factors used by the } \\
\text { modeling system. }\end{array}$ \\
\hline Apgemshelp.rtf & File that is displayed by the system when the Help option is selected. \\
\hline Fileinstructions.rtf & $\begin{array}{l}\text { File that is displayed by the system when the File Instructions option is } \\
\text { selected. }\end{array}$ \\
\hline Readme.rtf & File that is displayed by the "read me" viewer. \\
\hline Baemarb.dat & $\begin{array}{l}\text { Area-source input file used by the CALPUFF model. This file is generated } \\
\text { by the modeling system based upon user inputs. }\end{array}$ \\
\hline Lnemarb.dat & $\begin{array}{l}\text { Line-source input file used by the CALPUFF model. This file is generated } \\
\text { by the modeling system based upon user inputs. }\end{array}$ \\
\hline Ptemarb.dat & $\begin{array}{l}\text { Point-source input file used by the CALPUFF model. This file is generated } \\
\text { by the modeling system based upon user inputs. }\end{array}$ \\
\hline Ldustinp.txt & $\begin{array}{l}\text { Line and area-source input file used by the line dust emission model. This } \\
\text { file is generated by the modeling system based upon user input. }\end{array}$ \\
\hline Ldustout.txt & Output file generated by the line dust emission model. \\
\hline
\end{tabular}




\section{A.1.1 TerrFiles Directory}

The TerrFiles directory within the root DUSTRAN directory is used as a storage location for a default set of elevation and land-use files. These files are used by the TERREL and CTGPROC processors to generate the terrain and land-use input data required by the CALMET model. The default set of files present in the directory represents data sets for North America. The following table details the default set of files that are present in this directory.

\begin{tabular}{|l|l|}
\hline File Name & File Description \\
\cline { 1 - 2 } Nausgs2.img & $\begin{array}{l}\text { U.S. Geological Survey (USGS) Land Use/Land Cover Scheme; used as input to the } \\
\text { CTGPROC land-use processor for CALMET. }\end{array}$ \\
\cline { 1 - 2 } W100N40.dem & $\begin{array}{l}\text { Global topographic digital elevation models with a horizontal grid spacing of } \\
\text { W100N90.dem }\end{array}$ \\
\cline { 1 - 1 } W140N40.dem & $\begin{array}{l}\text { files are tiled for ease-of-distribution. The four files listed in this directory } \\
\text { spatially cover all of North America. }\end{array}$ \\
\cline { 1 - 2 } W140N90.dem
\end{tabular}

\section{A.1.2 ModelFiles Directory}

The ModelFiles directory within the root DUSTRAN directory is used to store default template input files that are used by the preprocessors, CALMET, CALPUFF, and CALGRID models. When a new site is added to DUSTRAN using the "Add Site" wizard, these files are automatically copied into the new site's StaticData directory. The following table lists and describes the files that are stored in the ModelFiles directory.

\begin{tabular}{|c|c|}
\hline File Name & File Description \\
\hline Cal.par & $\begin{array}{l}\text { Template copy of the default Cal.par file. The Cal.par file contains various model input } \\
\text { controls that are not available from within the DUSTRAN interface. This template file is } \\
\text { copied by the "Add Site" wizard and placed into the StaticData directory of the new site. }\end{array}$ \\
\hline CalGeo.inp & $\begin{array}{l}\text { CalGeo.inp is a template copy of the default input file for the TERREL, CTGPROC, and } \\
\text { MAKEGO terrain and land-use preprocessing programs in DUSTRAN. The file is } \\
\text { copied by the "Add Site" wizard and placed into the StaticData directory of the new site. }\end{array}$ \\
\hline Calgrid.inp & $\begin{array}{l}\text { Calgrid.inp is a template copy of the default input file for the CALGRID dispersion } \\
\text { model in DUSTRAN. The file is copied by the "Add Site" wizard and placed into the } \\
\text { StaticData directory of the new site. }\end{array}$ \\
\hline Calme & $\begin{array}{l}\text { Calmet.inp is a template copy of the default input file for the CALMET meteorological } \\
\text { processor in DUSTRAN. The file is copied by the "Add Site" wizard and placed into the } \\
\text { StaticData directory of the new site. }\end{array}$ \\
\hline Calpuff.inp & $\begin{array}{l}\text { Calpuff.inp is a template copy of the default input file for the CALPUFF dispersion } \\
\text { model in DUSTRAN. The file is copied by the "Add Site" wizard and placed into the } \\
\text { StaticData directory of the new site. }\end{array}$ \\
\hline Calpost.inp & $\begin{array}{l}\text { Calpost.inp is a template copy of the default input file for the CALPOST post-processor } \\
\text { in DUSTRAN. The file is copied by the "Add Site" wizard and placed in the StaticData } \\
\text { directory of the new site. }\end{array}$ \\
\hline Pgems3.par & $\begin{array}{l}\text { Template copy of the default Pgems } 3 \text {.par file used by the individual simulation sites. } \\
\text { The file is copied by the "Add Site" wizard and placed into the StaticData directory of } \\
\text { the new site. }\end{array}$ \\
\hline
\end{tabular}




\section{A.1.3 Site Directories}

When a new site is added through the "Add Site" wizard in DUSTRAN, a new directory is created for the site and is located within the DUSTRAN root directory. The site directory is used to store sitespecific model input and output files. In addition, the site directory stores site-specific data that are used by the models. Normally, the data are stored in sub-directories based upon the type of preprocessor or model that uses or generates the data. For example, data associated with the meteorological models and processors are stored in the MetData, MetOut, and MetRaw directories. The following table details the directories and files that are found within each site directory present in the modeling system.

\begin{tabular}{|c|c|}
\hline Name & Description \\
\hline MetData & $\begin{array}{l}\text { Directory used to store the meteorological data that have been processed for use in } \\
\text { a simulation. }\end{array}$ \\
\hline MetOut & $\begin{array}{l}\text { Directory used to store the output files generated by the CALMET meteorological } \\
\text { model. The files contain the gridded meteorological fields used by the CALPUFF } \\
\text { and CALGRID dispersion models. }\end{array}$ \\
\hline MetRaw & $\begin{array}{l}\text { Default directory used to store historic meteorological data for a site that may be } \\
\text { used in a simulation. }\end{array}$ \\
\hline RsData & $\begin{array}{l}\text { Directory used to store the run-specification files that are generated by DUSTRAN } \\
\text { for each simulation. }\end{array}$ \\
\hline StaticData & $\begin{array}{l}\text { Directory used to store the model-input template files as well as the data } \\
\text { associated with the sources and domains entered into the modeling system. }\end{array}$ \\
\hline TDMOut & $\begin{array}{l}\text { Directory used to store the data generated by the dispersion models during a } \\
\text { simulation run. }\end{array}$ \\
\hline TerData & $\begin{array}{l}\text { Directory that can be used to store terrain and land-use files that are specific to the } \\
\text { site. These files can be used instead of the default files found in the root TerrFiles } \\
\text { directory, providing the static Calgeo.inp file for the site is modified to reflect the } \\
\text { type and path of the new terrain files. }\end{array}$ \\
\hline TerOut & Directory used to store the data generated by the land-use and terrain processors. \\
\hline *.mdb & $\begin{array}{l}\text { Access database that stores information related to the site, such as source } \\
\text { information. }\end{array}$ \\
\hline *.mxd & ArcMap project file that stores the settings of the map display for the site. \\
\hline PGEMS3.ini & $\begin{array}{l}\text { Text file that stores the physical path of all the site-specific subdirectories list } \\
\text { above. }\end{array}$ \\
\hline
\end{tabular}

\section{A.1.3.1 MetData}

The MetData directory within each site directory is used to store the data that are used as input by the meteorological model and processors. The data found in this directory include upper-air observations, surface observations, and files containing station characteristics. 


\begin{tabular}{|c|c|}
\hline File Name & File Description \\
\hline Upn.dat & $\begin{array}{l}\text { Upper air station meteorological data file used as input by the CALMET model. } \\
\text { There will be one Upn.dat file present for each upper-air station selected for use } \\
\text { during the simulation run. }\end{array}$ \\
\hline Surf.dat & Surface-station meteorological data file used as input by the CALMET model. \\
\hline *.upr & $\begin{array}{l}\text { Upper-air-station meteorological data file generated from FSL data retrieved from } \\
\text { the NOAA website. }\end{array}$ \\
\hline *.unf & $\begin{array}{l}\text { This text file contains a listing of the upper-air stations available for a site as well as } \\
\text { descriptive information about each station. The station information found in this file } \\
\text { includes station name, station ID, UTM coordinate location, and longitude and } \\
\text { latitude of the station as well as the elevation. }\end{array}$ \\
\hline *.snf & $\begin{array}{l}\text { This text file contains a listing of the surface stations available for a site as well as } \\
\text { descriptive information about each station. The station information found within } \\
\text { this file includes station name, station ID, UTM coordinate location, and longitude } \\
\text { and latitude of the station as well as the elevation of the station. The .snf file also } \\
\text { contains the anemometer height used for the readings at the station. }\end{array}$ \\
\hline
\end{tabular}

\section{A.1.3.2 MetOut}

The MetOut directory within each site directory is used to store the different output files generated from the results of the meteorological model.

\begin{tabular}{|l|l|}
\hline File Name & File Description \\
\hline *.vec & $\begin{array}{l}\text { Comma-separated text file containing wind-vector data used to generate } \\
\text { the wind fields that are displayed within the ArcMap map display. }\end{array}$ \\
\hline WindBlownData.txt & $\begin{array}{l}\text { Comma-separated text file containing meteorological grid data pulled } \\
\text { from the output of CALMET. These data are required as input by the } \\
\text { wind-blown dust-emission model. }\end{array}$ \\
\hline *.dat & $\begin{array}{l}\text { Binary output data file generated by the CALMET meteorological } \\
\text { model. }\end{array}$ \\
\hline
\end{tabular}

\section{A.1.3.3 MetRaw}

The MetRaw directory within each site directory is used to store the historical meteorological data that are available to the modeling system for a specific site. Currently, the modeling system is set up to process historical data for the Yakima and Fort Irwin simulation sites. While this directory is provided by default for each site, users are given the ability to store historical meteorological data in the directory of their choice whether they are located in the site or DUSTRAN directories. Historical data found in this directory are normally site specific and will not transfer across sites.

\section{A.1.3.4 RsData}

The RsData directory within each site directory stores the run-specification file that is generated by DUSTRAN each time a site simulation is executed. Through this directory, each of the models is given access to the specification file to retrieve inputs entered by the user before the simulation starts. 


\begin{tabular}{|l|l|}
\hline File Name & File Description \\
\hline$*$.rs & $\begin{array}{l}\text { The run specification file is a comma-separated text file that contains the data } \\
\text { entered by the user through the user interface. }\end{array}$ \\
\hline
\end{tabular}

\section{A.1.3.5 StaticData}

The StaticData directory within each site directory contains the template input files that are used by the modeling system to create the actual input files used by the models and processors. The directory also contains the static parameter file Cal.par, which is used to hold model-run parameter data that are not accessible through the user interface. The Domain.dat and Sources.dat files are also stored within this directory and are used by the modeling system to track the characteristics of the domains and sources that have been entered for the site. The following table provides the details of the files that are stored within the StaticData directory.

\begin{tabular}{|l|l|}
\hline File Name & File Description \\
\hline CalGeo.inp & $\begin{array}{l}\text { Text file containing input sections for the Terrel, Ctgproc, and Makegeo } \\
\text { processors. These input sections contain the default values for the individual } \\
\text { processors and are used in the creation of the actual input file required for } \\
\text { running the processors. }\end{array}$ \\
\hline CALGRID.inp & $\begin{array}{l}\text { Text file containing the default input parameters for the CALGRID model. } \\
\text { This file is used in the creation of the actual input file for executing the } \\
\text { CALGRID model for a simulation. }\end{array}$ \\
\hline CALMET.inp & $\begin{array}{l}\text { Text file containing the default input parameters for the CALMET model. } \\
\text { This file is used in the creation of the actual input file for executing the } \\
\text { CALMET model for a simulation. }\end{array}$ \\
\hline CALPUFF.inp & $\begin{array}{l}\text { Text file containing the default input parameters for the CALPUFF model. } \\
\text { This file is used in the creation of the actual input file for executing the } \\
\text { CALPUFF model for a simulation. }\end{array}$ \\
\hline Cal.par & $\begin{array}{l}\text { A static parameter file that contains inputs that are normally not changed } \\
\text { (i.e., are considered static) for a modeling run, but can be modified for testing } \\
\text { or fine tuning of the modeling system. }\end{array}$ \\
\hline StationAlias.txt & $\begin{array}{l}\text { Text file containing alias information for the National Training Center (NTC) } \\
\text { surface station data. Currently, this file is only used for the Fort Irwin site. }\end{array}$ \\
\hline
\end{tabular}

\section{A.1.3.6 TDMOut}

The TDMOut directory within each site directory is used to store the output data generated by the CALGRID and CALPUFF dispersion models. The directory also contains the contouring text files that are generated by the CalConc processor based on the results generated by the dispersion models. Data contained within the .ccn and .crd files are used by the modeling system to create the contours that are shown on the DUSTRAN map display following the successful execution of a simulation. The file types that are found within this directory are listed in the following table. 


\begin{tabular}{|c|c|}
\hline File Type & File Type Description \\
\hline$* . \operatorname{ccn}$ & $\begin{array}{l}\text { Text file containing the concentration contour data generated from the output } \\
\text { files of the CALGRID or CALPUFF models. }\end{array}$ \\
\hline$* . \mathrm{CON}$ & Binary output file generated by the CALPUFF model. \\
\hline$*$. crd & Text file containing the contouring grid coordinates. \\
\hline *.dat & Binary output file generated by the CALGRID model. \\
\hline *.dep & $\begin{array}{l}\text { Text file containing the deposition contour data generated from the output files } \\
\text { of the CALGRID or CALPUFF models. }\end{array}$ \\
\hline$* . \exp$ & $\begin{array}{l}\text { Text file containing the exposure contour data generated from the output files of } \\
\text { the CALGRID or CALPUFF models. }\end{array}$ \\
\hline$* . \operatorname{tdp}$ & $\begin{array}{l}\text { Text file containing the total deposition contour data generated from the output } \\
\text { files of the CALGRID or CALPUFF models. }\end{array}$ \\
\hline
\end{tabular}

\section{A.1.3.7 TerOut}

The TerOut directory within each site directory is used to store the output generated by the three terrain processors used by the modeling system. Output from the TERREL, CTGPROC, and MAKEGEO processors is stored within this directory following each successful simulation executed by the modeling system. The following table details the files that are stored within this directory.

\begin{tabular}{|l|l|}
\hline File Type & File Type Description \\
\hline *.out & Output file generated by the TERREL processor. \\
\hline *.dat & Output files generated by the CTGPROC and MAKEGEO processors. \\
\hline
\end{tabular}

\section{A.2 Site Directory Static Data Files}

\section{A.2.1 Cal.par File}

Several parameters used by the DUSTRAN modeling system are not directly accessible through the DUSTRAN interface. Instead, these parameters are stored in a text file called "cal.par." These parameters change infrequently and were intentionally omitted from the interface to minimize screen clutter and accidental user revisions.

The cal.par file is an ASCII, comma-delimited text file and can be opened in any standard text editor. The file is stored in the "StaticData" directory for each site, and the values in the file apply to that site. For the most part, the file is self-describing; variable-specific comments are provided before or on the line of the actual variable.

A sample cal.par file is provided in the following section. For clarity, each line (excluding lines that wrap) is numbered within the \{\} brackets. The line numbers are used to reference the table in Section A.2.1.2, which provides a detailed description of each line in the sample file. The \{\} brackets and line numbers do not exist in an actual cal.par file. Items in bold are comments and are not used by the code. 


\section{A.2.1.1 Example Cal.par File}

$\{1\}$ Meteorological parameters to construct profile from single observation and construct surface met file

$\{2\}$ [WS, WD, Stability specified through single observation input window within DUSTRAN interface]

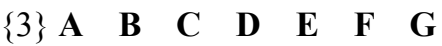

\{4\} $0.07,0.07,0.10,0.15,0.35,0.35,0.35$,Prural [Power Law exponents for wind profile; currently used]

$\{5\} \quad 0.15,0.15,0.20,0.25,0.30,0.30,0.30$, Purban [Power Law exponents for wind profile]

$\{6\}-0.025,-0.020,-0.015,-0.010,0.010,0.025,0.040, \mathrm{DtDz}$ [Temperature lapse rate vs. stability]

$\{7\}$ 0.,20.,50.,100.,500.,2000.,9000., zface heights used in the Calmet, Calpuff, and Calgrid models

$\{8\} 10,0 \mathbf{Z 0}$, ZTOP [Height of bottom and top sounding values (m agl)]

$\{9\} 11$, nHts [Number of heights in sounding]

$\{10\}$ 100,ICEIL [Ceiling height (hundreds of feet)]

$\{11\}$ 0,ICC [Opaque sky cover (tenths)]

$\{12\}$ 25,TEMPK [Surface air temperature (Deg C)]

$\{13\}$ 50,IRH [Relative humidity (percent)]

$\{14\}$ 1000,PRES [Station pressure (mb)]

$\{15\} 1000$, WATDENSE [Density of water $\left.\left(\mathbf{g} / \mathbf{m}^{\wedge} \mathbf{3}\right)\right]$

$\{16\}$ 0,IPCODE [Precipitation code ( $0=$ =no precip; 1-18=liquid; 19-45=frozen)]

$\{17\}$ T,WSPROF [T - Power law profile; $\mathbf{F}$ - Constant wind speed with height]

$\{18\}$ F,MM5 data used check

$\{19\}$ F,Advanced user Deposition flag; if T, then the ability to set deposition velocities is turned on

$\{20\} 50,50$, Number of $\mathbf{x}$ and $y$ grid points for CALPUFF run

$\{21\} 20,20$, Number of $\mathbf{x}$ and $\mathbf{y}$ grid points for CALGRID run

$\{22\}$ Specie names, molecular weight, particle mean diameter, particle standard deviation, default userdefined deposition velocity

$\{23\}$ 4,NumSpecs [Number of species present] Molecular weight does not appear to be used by Calpuff

$\{24\}$ PM10,0.0,10.0,1.0,0.0

$\{24\}$ PM2.5,0.0,2.5,1.0,0.0

$\{24\}$ PM15,0.0,15.0,1.0,0.0

$\{24\}$ PM30,0.0,30.0,1.0,0.0

$\{25\}$ Point Source Parameter Units, 8 ,

$\{26\}$ Parameter,Release Height,Exit Velocity,Exit Temperature,Stack Diameter,Building Cross Section,Initial Horizontal Plume Size,Initial Vertical Plume Size,Release Rate,

$\{27\}$ Default Value, $0,0,25,1,0,1,1,1$,

$\{28\}$ User Units, $\mathrm{m}, \mathrm{m} / \mathrm{s}, \mathrm{C}, \mathrm{m}, \mathrm{m}^{\wedge} 2, \mathrm{~m}, \mathrm{~m}, \mathrm{~g} / \mathrm{s}$,

$\{29\}$ Model Units, m,m/s, C,m, $\mathrm{m}^{\wedge} 2, \mathrm{~m}, \mathrm{~m}, \mathrm{~g} / \mathrm{s}$,

$\{30\}$ Conversion Factor, $1,1,1,1,1,1,1,1$,

$\{31\}$ Min Value, 0,0, inf, $0,0,1,1,0$,

\{32\} Max Value,inf,inf,inf,inf,inf,inf,inf,inf,

\{33\} Range String,Release Height $>=0.0$,Exit Velocity $>=0.0$, No Boundaries,Stack Diameter $>=0$, Building Cross Section $>=0.0$, Initial Horizontal Plume Size 0.0, Initial Vertical Plume Size $>0.0$, Release Rate $>=0.0$,

$\{34\}$ Line Source Parameter Units,3,

\{35\} Parameter,HTL,ELEVL,QEMITL,

$\{36\}$ Default Value, $0,0,0$,

$\{37\}$ User Units,m,m,g/s/m,

$\{38\}$ Model Units,m,m,g/s/m,

\{39\} Conversion Factor, 1,1,1,

$\{40\}$ Min Value, $0,0,0$,

\{41\} Max Value,inf,inf,inf,

$\{42\}$ Range String,HTL $>=0.0$, ELEVL $>=0.0$, QEMITL $>=0.0$, 
$\{43\}$ Area Source Parameter Units, 7 ,

\{44\} Parameter,HT,ELEV,TEMPK,WEFF,REFF,SIGZ,QEMIT,

$\{45\}$ Default Value, $0,0,25,1,1,1$,

$\{46\}$ User Units,m,m,C,m/s,m,m,g/s

$\{47\}$ Model Units, m, m,K,m/s,m,m,g/s

$\{48\}$ Conversion Factor, $1,1,1 \& 0,1,1,1,1$,

$\{49\}$ Min Value, 0,0, inf, $1,1,1,0$,

$\{50\}$ Max Value,inf,inf,inf,inf,inf,inf,inf,

$\{51\}$ Range String, HT $>=0.0, \mathrm{ELEV}>=0.0$, No Boundaries, WEFF $>=1.0, \mathrm{REFF}>=1.0, \mathrm{SIGZ}>=1.0, \mathrm{QEMIT}>=$ 0.0 ,

$\{52\}$ Meteorology Parameter Units,3,

$\{53\}$ Parameter,Wind Speed,Wind Direction,Mixing Height,

$\{54\}$ Default Value, 2.2,270,500,

$\{55\}$ User Units, $\mathrm{m} / \mathrm{s}$, No Unit,m,

$\{56\}$ Model Units,m/s,No Unit,m,

$\{57\}$ Conversion Factor, $1,1,1$,

$\{58\}$ Min Value, $0,0,0$,

\{59\} Max Value,inf,360,inf,

$\{60\}$ Range String,Wind Speed $>=0.0$,Wind Direction $>=0$ and Wind Direction $<=360$,Mixing Height $>=0.0$,

$\{61\}$ Line Source Vehicle Parameter,4,

$\{62\}$ Parameter,Vehicle SpeedL,Vehicle WeightL,Road LengthL,Distance TraveledL,

$\{63\}$ Default Value,50,3000,0.0,0.0,

$\{64\}$ User Units,km/hr,kg,km,km,

$\{65\}$ Model Units,km/hr,kg,km,km,

$\{66\}$ Conversion Factor, $1,1,1,1$,

$\{67\}$ Min Value, $0,0,0,0$,

$\{68\}$ Max Value,inf,inf,inf,inf,

$\{69\}$ Range String,Vehicle Speed $>=0.0$,Vehicle Weight $>=0.0$, Road Length $>=0.0$,Distance Traveled $>=0.0$,

$\{70\}$ Area Source Vehicle Parameter,4,

$\{71\}$ Parameter,Vehicle Speed,Vehicle Weight,Polygon Area,Distance Traveled,

$\{72\}$ Default Value, 50,3000,0.0,0.0,

$\{73\}$ User Units, $\mathrm{km} / \mathrm{hr}, \mathrm{kg}, \mathrm{km}^{\wedge} 2, \mathrm{~km}$,

$\{74\}$ Model Units,km/hr,kg,km^2,km,

$\{75\}$ Conversion Factor, $1,1,1,1$,

$\{76\}$ Min Value, $0,0,0,0$,

$\{77\}$ Max Value,inf,inf,inf,inf,

$\{78\}$ Range String,Vehicle Speed $>=0.0$,Vehicle Weight $>=0.0$, Polygon Area $>0.0$, Distance Traveled $>=0.0$,

$\{79\} 1.0 \mathrm{E}+6$, Contours (micrograms $/ \mathrm{m}^{\wedge} 3$ ), Unit label and conversion factor for the concentration contour labels

$\{80\} 1.0 \mathrm{E}+6$, Contours (micrograms $/ \mathrm{m}^{\wedge} 2 / \mathrm{sec}$ ), Unit label and conversion factor for the deposition contour labels

$\{81\} 1.0 \mathrm{E}+6$, Contours (micrograms-sec $/ \mathrm{m}^{\wedge} 3$ ), Unit label and conversion factor for the exposure contour labels

$\{82\} 1.0 \mathrm{E}+6$, Contours (micrograms $/ \mathrm{m}^{\wedge} 2$ ), Unit label and conversion factor for the total deposition contours

$\{83\}$ 1.0E-15,Minimum contour level used by Calconc suggest 3 orders of magnitude smaller than the smallest specified contour level

$\{84\} 12$, Number of contour levels to be calculated

$\{85\} 1.0 \mathrm{E}-12$

$\{85\} 1.0 \mathrm{E}-11$

$\{85\} 1.0 \mathrm{E}-10$

$\{85\} 1.0 \mathrm{E}-9$

$\{85\} 1.0 \mathrm{E}-8$

$\{85\} 1.0 \mathrm{E}-7$

$\{85\} 1.0 \mathrm{E}-6$ 
$\{85\} \quad 1.0 \mathrm{E}-5$

$\{85\} 1.0 \mathrm{E}-4$

$\{85\} 1.0 \mathrm{E}-3$

$\{85\} 1.0 \mathrm{E}-2$

$\{85\} 1.0 \mathrm{E}-1$

\{86\} 10,NUMVEHCODES [Number of vehicle integer codes]

\{87\} 1,1176,Dodge Neon, 2002 Civilian vehicle with Eagle GA Touring M+S P185/165R 85T tires

$\{87\}$ 2,1759,Dodge Caravan,2002 Civilian vehicle with GoodYear Integrity M+S 215/70R15 98S tires

\{87\} 3,1516,Ford Taurus,2002 Civilian vehicle with Firestone M+S P215/60R16 94T tires

$\{87\} 4,3100$, GMC G20 Van, DRI TRAKER vehicle used for measuring dust emissions in real time.

\{87\} 5,5227,GMC C5500, 1999 Civilian vehicle 6 wheels with GoodYear and Michelin tires

\{87\} 6,2445,HMMWV, Military Vehicle 4 wheels with tires

\{87\} 7,14318,M923A2 (5-Ton), Military Vehicle 2 front wheels and 8 rear wheels on dual axles

$\{87\}$ 8,8060,M1078 LMTV, 2.5 Ton Military vehicle with 4 wheels and tires

$\{87\}$ 9,17727,M977 HEMMET, Military vehicle with 8 wheels and tires

$\{87\}$ 10,23636,Freightliner, Tractor trailer rig with 22 wheels and tires

$\{88\}$ Calpuff polar grid information

$\{89\} 1$

$\{90\}$ POLAR_GRD

$\{91\}$ 36,10,200.,400.,800.,1500.,2500.,3500.,4500.,5500.,6500.,7500.,

$\{92\} 3,1000$,number of grid spacings used to create area source grids - resolution of area source grid

(*** Note Calpuff will only accept up to 4000 discrete receptors $* * *$ )

$\{93\} 2,2$, Wind vector resolution, max windspeed

$\{94\} 1.0 \mathrm{E}-20$, Initial concentration value for CalGrid model

$\{95\}$, Model run flag $\mathbf{1}=$ Calpuff run $\mathbf{2}=$ Calgrid run $3=$ Both models run

$\{96\} 1$, Run Calgrid in wind blown dust mode $1=$ Run wind blown $\mathbf{0}=$ Run without windblown dust

$\{97\}$ 0.0,soil moisture (water mass / soil mass)

$\{98\} 12,12,12$,surface silt loading $\left(\mathbf{g ~ m}^{-2}\right)$, surface silt content $(\%)$, surface moisture content $(\%)$

$\{99\} \mathrm{http} / / /$ www.irwin.army.mil/weather/WXdata.txt

$\{100\}$

http://raob.fsl.noaa.gov/intl/GetRaobs.cgi?shour=All+Times\&ltype=All+Levels\&wunits=Knots\&access=WBAN+St ation + Identifier\&view $=$ NO\&osort=Station + Series + Sort\&oformat $=\mathrm{FSL}+$ format $+(\mathrm{ASCII}+$ text $)$

\section{A.2.1.2 Cal.par File Description}

\begin{tabular}{|l|l|l|}
\hline $\begin{array}{c}\text { Line } \\
\text { Number }\end{array}$ & \multicolumn{1}{|c|}{ Data Type } & Description \\
\hline 1 & String & Wind profile description header. \\
\hline 2 & String & Wind profile description header. \\
\hline 3 & String & Wind profile description header. \\
\hline 4 & Floating Point & $\begin{array}{l}\text { Rural Power Law exponents for wind profile. The wind profile is generated } \\
\text { for simulations using the single observation meteorology option. (defaults } \\
\text { Pr1=0.07, Pr2=0.07, Pr3=0.10, Pr4=0.15, Pr5=0.35, Pr6=0.35, Pr7=0.35) }\end{array}$ \\
\hline 5 & Floating Point & $\begin{array}{l}\text { Urban Power Law exponents for wind profile. The wind profile is generated } \\
\text { for simulations using the single observation meteorology option. (defaults } \\
\text { Pu1=0.15, Pu2=0.15, Pu3=0.20, Pu4=0.25, Pu5=0.30, Pu6=0.30, Pu7=0.30) }\end{array}$ \\
\hline 6 & Floating Point & $\begin{array}{l}\text { Potential temperature lapse rate vs. stability for wind profile. The profile is } \\
\text { generated using the single observation meteorology option. (defaults Pt1=- } \\
0.025, \text { Pt2=-0.20, Pt3=-0.015, Pt4=-0.010, Pt5=0.010, Pt6=0.025, Pt7=0.40) }\end{array}$ \\
\hline
\end{tabular}




\begin{tabular}{|c|c|c|}
\hline $\begin{array}{c}\text { Line } \\
\text { Number }\end{array}$ & Data Type & Description \\
\hline 7 & String & $\begin{array}{l}\text { Comma-separated list of heights (meters above ground) for CALMET's } \\
\text { vertical grid. The first value is the height of the bottom face of the } 1^{\text {st }} \text { grid cell } \\
\text { and the last height is the height of the top face of the highest grid cell. The } \\
\text { CALMET-derived parameters occur at the mid-point between successive } \\
\text { heights. The number of heights is determined from the number of values } \\
\text { listed minus one (the zero value). }\end{array}$ \\
\hline 8 & Floating Point & $\begin{array}{l}\text { Height (meters above ground level [agl]) of lowest observation in upper-air } \\
\text { observation profile for "Single Observation" meteorology }\end{array}$ \\
\hline 8 & Floating Point & $\begin{array}{l}\text { Height (meters agl) of the top observation in the upper-air observation profile } \\
\text { for "Single Observation" meteorology. If ZTOP equals } 0.0 \text {, then the default } \\
\text { value found in the static Calmet.inp file is used. }\end{array}$ \\
\hline 9 & Integer & $\begin{array}{l}\text { Number of heights in the upper-air observation profile, for "Single } \\
\text { Observation" meteorology. }\end{array}$ \\
\hline 10 & Integer & $\begin{array}{l}\text { Default ceiling height (hundreds of feet), for "Single Observation" } \\
\text { meteorology. }\end{array}$ \\
\hline 11 & Integer & Default opaque sky cover (tenths), for "Single Observation" meteorology. \\
\hline 12 & Floating Point & $\begin{array}{l}\text { Default surface air temperature in (deg C), for "Single Observation" } \\
\text { meteorology. }\end{array}$ \\
\hline 13 & Integer & Default relative humidity (\%), for "Single Observation" meteorology. \\
\hline 14 & Floating Point & Default station pressure (mb), for "Single Observation" meteorology. \\
\hline 15 & Floating Point & $\begin{array}{l}\text { Density of water }\left(\mathrm{kg} / \mathrm{m}^{3}\right) \text {. Used in the conversion of AGDISP output to } \\
\text { emission rates. }\end{array}$ \\
\hline 16 & Integer & Precipitation code $(0=$ no precipitation, $1-18=$ liquid, $19-45=$ frozen $)$. \\
\hline 17 & $\begin{array}{l}\text { Character } \\
\text { (True / False) }\end{array}$ & $\begin{array}{l}\text { Power law profile flag }(T=\text { Power law profile, } F=\text { Constant wind speed with } \\
\text { height }) .[(\text { default } T])\end{array}$ \\
\hline 18 & $\begin{array}{l}\text { Character } \\
\text { (True / False) }\end{array}$ & $\begin{array}{l}\text { Flag designating whether or not MM5 data are to be used by the modeling } \\
\text { system. }\end{array}$ \\
\hline 19 & $\begin{array}{l}\text { Character } \\
\text { (True / False) }\end{array}$ & $\begin{array}{l}\text { Flag designating whether to turn on the advanced user parameters for the } \\
\text { calculation of deposition. If this flag }=\mathrm{T} \text {, then the user has the ability to enter } \\
\text { geometric standard deviations and deposition velocities. }\end{array}$ \\
\hline 20 & Integer & $\begin{array}{l}\text { Grid spacing and number of receptor locations in the } \mathrm{X} \text { and } \mathrm{Y} \text { directions for a } \\
\text { CALPUFF simulation. }\end{array}$ \\
\hline 21 & Integer & $\begin{array}{l}\text { Grid spacing and number of receptors locations in the } \mathrm{X} \text { and } \mathrm{Y} \text { directions for a } \\
\text { CALGRID simulation. }\end{array}$ \\
\hline 22 & String & Specie data header. \\
\hline 23 & Integer & Number of species available in DUSTRAN. \\
\hline 24 & String & $\begin{array}{l}\text { Name of the specie, molecular weight, particle mean diameter, particle } \\
\text { standard deviation, default deposition velocity }\end{array}$ \\
\hline 25 & Integer & Number of parameters used by a point source \\
\hline 26 & String & Label strings for point-source parameters separated by commas. \\
\hline 27 & String & Default values for point-source parameters separated by commas. \\
\hline 28 & String & User units for point-source parameters separated by commas. \\
\hline 29 & String & Model units for point-source parameters separated by commas. \\
\hline
\end{tabular}




\begin{tabular}{|c|c|c|}
\hline $\begin{array}{c}\text { Line } \\
\text { Number }\end{array}$ & Data Type & Description \\
\hline 30 & String & Conversion values for point-source parameters separated by commas. \\
\hline 31 & String & Minimum values for point-source parameters separated by commas. \\
\hline 32 & String & Maximum values for point-source parameters separated by commas. \\
\hline 33 & String & $\begin{array}{l}\text { Strings giving the range of values that are appropriate for point-source } \\
\text { parameters separated by commas. }\end{array}$ \\
\hline 34 & Integer & Number of parameters used by a line source. \\
\hline 35 & String & Label strings for line-source parameters separated by commas. \\
\hline 36 & String & Default values for line-source parameters separated by commas. \\
\hline 37 & String & User units for line-source parameters separated by commas. \\
\hline 38 & String & Model units for line-source parameters separated by commas. \\
\hline 39 & String & Conversion values for line-source parameters separated by commas. \\
\hline 40 & String & Minimum values for line-source parameters separated by commas. \\
\hline 41 & String & Maximum values for line-source parameters separated by commas. \\
\hline 42 & String & $\begin{array}{l}\text { Strings giving the range of values that are appropriate for line-source } \\
\text { parameters separated by commas. }\end{array}$ \\
\hline 43 & Integer & Number of parameters used by an area source. \\
\hline 44 & String & Label strings for area-source parameters separated by commas. \\
\hline 45 & String & Default values for area-source parameters separated by commas. \\
\hline 46 & String & User units for area-source parameters separated by commas. \\
\hline 47 & String & Model units for area-source parameters separated by commas. \\
\hline 48 & String & Conversion values for area-source parameters separated by commas. \\
\hline 49 & String & Minimum values for area-source parameters separated by commas. \\
\hline 50 & String & Maximum values for area-source parameters separated by commas. \\
\hline 51 & String & $\begin{array}{l}\text { Strings giving the range of values that are appropriate for area-source } \\
\text { parameters separated by commas. }\end{array}$ \\
\hline 52 & Integer & Number of parameters used for meteorology input. \\
\hline 53 & String & Label strings for meteorology input parameters separated by commas. \\
\hline 54 & String & Default values for meteorology input parameters separated by commas. \\
\hline 55 & String & User units for meteorology input parameters separated by commas. \\
\hline 56 & String & Model units for meteorology input parameters separated by commas. \\
\hline 57 & String & Conversion values for meteorology input parameters separated by commas. \\
\hline 58 & String & Minimum values for meteorology input parameters separated by commas. \\
\hline 59 & String & Maximum values for meteorology input parameters separated by commas. \\
\hline 60 & String & $\begin{array}{l}\text { Strings giving the range of values that are appropriate for meteorology input } \\
\text { parameters separated by commas. }\end{array}$ \\
\hline 61 & Integer & Number of parameters used for line-source vehicle input. \\
\hline 62 & String & Label strings for line-source vehicle input parameters separated by commas. \\
\hline 63 & String & Default values for line-source vehicle input parameters separated by commas. \\
\hline 64 & String & User units for line-source vehicle input parameters separated by commas. \\
\hline 65 & String & Model units for line-source vehicle input parameters separated by commas. \\
\hline 66 & String & $\begin{array}{l}\text { Conversion values for line-source vehicle input parameters separated by } \\
\text { commas. }\end{array}$ \\
\hline 67 & String & Min. values for line-source vehicle input parameters separated by commas. \\
\hline
\end{tabular}




\begin{tabular}{|c|c|c|}
\hline $\begin{array}{c}\text { Line } \\
\text { Number }\end{array}$ & Data Type & Description \\
\hline 68 & String & $\begin{array}{l}\text { Maximum values for line-source vehicle input parameters separated by } \\
\text { commas. }\end{array}$ \\
\hline 69 & String & $\begin{array}{l}\text { Strings giving the range of values that are appropriate for line-source vehicle } \\
\text { input parameters separated by commas. }\end{array}$ \\
\hline 70 & Integer & Number of parameters used for area-source vehicle input. \\
\hline 71 & String & Label strings for area-source vehicle input parameters separated by commas. \\
\hline 72 & String & Default values for area-source vehicle input parameters separated by commas. \\
\hline 73 & String & User units for area-source vehicle input parameters separated by commas. \\
\hline 74 & String & Model units for area-source vehicle input parameters separated by commas. \\
\hline 75 & String & $\begin{array}{l}\text { Conversion values for area-source vehicle input parameters separated by } \\
\text { commas. }\end{array}$ \\
\hline 76 & String & $\begin{array}{l}\text { Minimum values for area-source vehicle input parameters separated by } \\
\text { commas. }\end{array}$ \\
\hline 77 & String & $\begin{array}{l}\text { Maximum values for area-source vehicle input parameters separated by } \\
\text { commas. }\end{array}$ \\
\hline 78 & String & $\begin{array}{l}\text { Strings giving the range of values that are appropriate for area-source vehicle } \\
\text { input parameters separated by commas. }\end{array}$ \\
\hline 79 & $\begin{array}{l}\text { Floating Point, } \\
\text { String }\end{array}$ & $\begin{array}{l}\text { Conversion factor for the concentration contour labels, Unit label for the } \\
\text { concentration contour labels }\end{array}$ \\
\hline 80 & $\begin{array}{l}\text { Floating Point, } \\
\text { String }\end{array}$ & $\begin{array}{l}\text { Conversion factor for the deposition contour labels, Unit label for the } \\
\text { deposition contour labels. }\end{array}$ \\
\hline 81 & $\begin{array}{l}\text { Floating Point, } \\
\text { String }\end{array}$ & $\begin{array}{l}\text { Conversion factor for the exposure contour labels, Unit label for the exposure } \\
\text { contour labels }\end{array}$ \\
\hline 82 & $\begin{array}{l}\text { Floating Point, } \\
\text { String }\end{array}$ & $\begin{array}{l}\text { Conversion factor for the total deposition contour labels, Unit label for the } \\
\text { total deposition contour labels }\end{array}$ \\
\hline 83 & Floating Point & $\begin{array}{l}\text { Minimum concentration level to be used in the processing of the contours } \\
\text { from the generated results. }\end{array}$ \\
\hline 84 & Integer & The number of contour levels to be processed from the generated results. \\
\hline 85 & Floating Point & $\begin{array}{l}\text { The individual contour levels to be used in the processing of the generated } \\
\text { results. }\end{array}$ \\
\hline 86 & Integer & Number of vehicles available in the modeling system. \\
\hline 87 & $\begin{array}{l}\text { Integer, Floating } \\
\text { Point, String, } \\
\text { String }\end{array}$ & Vehicle ID number, Vehicle weight, Vehicle name, Vehicle description \\
\hline 88 & String & Polar grid header line. \\
\hline 89 & Integer & Number of polar grids to be used in the simulation run. \\
\hline 90 & String & Name of the polar grid to be used in the simulation run. \\
\hline 91 & $\begin{array}{l}\text { Integer, Integer, } \\
\text { Floating Point }\end{array}$ & $\begin{array}{l}\text { Number of radial distances used in the generation of the point-source polar } \\
\text { grids, Number of arc distances (NARCS) used in the generation of the point- } \\
\text { source polar grids, Comma-separated list of arc distances used in the } \\
\text { generation of the point-source polar grids (there must be NARCS present in } \\
\text { the file) }\end{array}$ \\
\hline
\end{tabular}




\begin{tabular}{|c|c|c|}
\hline $\begin{array}{c}\text { Line } \\
\text { Number }\end{array}$ & Data Type & Description \\
\hline 92 & Integer, Integer & $\begin{array}{l}\text { Parameters used to specify "sub-grid" Cartesian receptor grids. The first is the } \\
\text { number of "main" Cartesian grid points extending beyond the area-source } \\
\text { boundaries, and the second number is the spacing }(\mathrm{m}) \text { between the "sub-grid" } \\
\text { receptor points. }\end{array}$ \\
\hline 93 & $\begin{array}{l}\text { Integer, Floating } \\
\text { Point }\end{array}$ & $\begin{array}{l}\text { Two parameters for setting the resolution of the wind vector field displayed on } \\
\text { the map and for setting the maximum wind speed used in the creation of the } \\
\text { wind vector field. The first parameter indicates to display every " } \mathrm{n} \text { "th" vector, } \\
\text { and the second parameter is the magnitude of the vector spanning the distance } \\
\text { between the vector output grid points. }\end{array}$ \\
\hline 94 & Floating Point & Initial concentration value to be used by the CALGRID model. \\
\hline 95 & Integer & $\begin{array}{l}\text { Flag used to determine which dispersion model to execute for the simulation } \\
\text { run. ( } 1=\text { CALPUFF run } 2=\text { CALGRID run } 3=\text { Both models run). Currently, } \\
\text { model selection is being handled by the interface (i.e., Source Emissions = } \\
\text { CALPUFF; Windblown Dust = CALGRID) and not through this flag. }\end{array}$ \\
\hline 96 & Integer & $\begin{array}{l}\text { Flag used to determine whether the CALGRID run is to be run in the } \\
\text { windblown mode. ( } 1=\text { Run wind-blown model } 0=\text { Run CALGRID without } \\
\text { windblown dust) }\end{array}$ \\
\hline 97 & Floating Point & Soil moisture used in wind-blown dust calculation. (water mass/soil mass) \\
\hline 98 & Floating Point & $\begin{array}{l}\text { Road surface characteristics for AP-42 calculations of dust from vehicle } \\
\text { activity. Surface silt loading }\left(\mathrm{g} \mathrm{m}^{-2}\right) \text { for paved roads; surface material silt } \\
\text { content }(\%) \text { and surface material moisture content }(\%) \text { for unpaved roads. }\end{array}$ \\
\hline 99 & String & $\begin{array}{l}\text { URL address of the website used to retrieve surface meteorological data for Ft. } \\
\text { Irwin site if data for desired period not in local DUSTRAN directory. }\end{array}$ \\
\hline 100 & String & $\begin{array}{l}\text { URL address of the website used to retrieve upper air station data for Ft. Irwin } \\
\text { site if data for desired period not in local DUSTRAN directory. }\end{array}$ \\
\hline
\end{tabular}

\section{A.2.2 Calmet.inp}

The CALMET input file (Calmet.inp) is located in the "StaticData" directory for each site within DUSTRAN and is the template input file for CALMET. Its contents are merged with select-user input from the DUSTRAN interface for each simulation. Many of the parameters contained within Calmet.inp control certain aspects of the CALMET-derived gridded wind field and boundary-layer parameters. These parameters are not available from the DUSTRAN interface and can only be edited via the static file.

The static Calmet.inp file normally contains the most ideal parameter settings for a given site. However, there may be times when the user wants to examine the effects of modifying certain parameter settings. Extreme caution should be used when changing any parameter in the static Calmet.inp file, as it may cause unrealistic results. Each parameter in the Calmet.inp is documented in detail in the CALMET user's guide (Scire et. al. 2000b). The following discussion highlights those parameters that are generally considered the most important relative to affecting the gridded fields. Generally, these parameters are from the section "INPUT GROUP 5 - Wind Field Options and Parameters," and control the threedimensional gridded wind field that is derived from surface and upper-air observations. 


\begin{tabular}{|c|c|c|c|}
\hline $\begin{array}{l}\text { CALMET } \\
\text { Parameter }\end{array}$ & $\begin{array}{c}\text { Allowed } \\
\text { Values }\end{array}$ & $\begin{array}{l}\text { Recommended } \\
\text { Value }\end{array}$ & Description \\
\hline IWFCOD & $\begin{array}{c}0(\mathrm{No}) \\
\text { or } \\
1 \text { (Yes) }\end{array}$ & 1 (Yes) & $\begin{array}{l}\text { Wind Field Model Option-This parameter allows the } \\
\text { CALMET model to be run as a diagnostic model and } \\
\text { include the effects of terrain, or only perform objective } \\
\text { analysis on the observations without regards to the } \\
\text { underlying terrain. It is recommend the diagnostic } \\
\text { model be used (IWFCOD = 1). The objective analysis } \\
\text { might be considered if there are a high number of met. } \\
\text { stations in an otherwise relatively flat domain. }\end{array}$ \\
\hline IKINE & $\begin{array}{c}0(\mathrm{No}) \\
\text { or } \\
1 \text { (Yes) }\end{array}$ & 0 (No) & $\begin{array}{l}\text { Compute Kinematic effects-This parameter is } \\
\text { intended to provide a characterization of terrain effects } \\
\text { on vertical wind speed. CALMET then adjusts the } \\
\text { horizontal wind speed components for mass continuity. } \\
\text { This parameter has been found to cause unrealistic } \\
\text { results in the horizontal wind field and so use of this } \\
\text { parameter is discouraged. }\end{array}$ \\
\hline IFRADJ & $\begin{array}{c}0(\mathrm{No}) \\
\text { or } \\
1 \text { (Yes) }\end{array}$ & 1 (Yes) & $\begin{array}{l}\text { Compute Froude number adjustment-This parameter } \\
\text { is used to calculate the effects of terrain blocking on the } \\
\text { horizontal wind field. A Froude number is calculated at } \\
\text { each grid node in CALMET and if the value is below a } \\
\text { certain criteria, the flow is considered blocked and is } \\
\text { modified tangent to the terrain. The use of this } \\
\text { parameter is recommended, particularly for domains in } \\
\text { complex terrain. }\end{array}$ \\
\hline ISLOPE & $\begin{array}{c}0(\mathrm{No}) \\
\text { or } \\
1 \text { (Yes) }\end{array}$ & 1 (Yes) & $\begin{array}{l}\text { Compute slope flow effects-This parameter calculates } \\
\text { the wind flows created by sloping terrain, such as cold- } \\
\text { air drainage flows. The use of this parameter is } \\
\text { recommended, particularly for domains in complex } \\
\text { terrain under calm, stable conditions. }\end{array}$ \\
\hline IEXTRP & $\begin{array}{c}1(-1) \\
\text { or } \\
2(-2) \\
\text { or } \\
3(-3) \\
\text { or } \\
4(-4)\end{array}$ & $\begin{array}{l}-2 \\
\text { or } \\
-4\end{array}$ & $\begin{array}{l}\text { Extrapolate surface wind observations to upper levels- } \\
\text { Surface winds can be extrapolated into the vertical to } \\
\text { augment upper-air data when creating the three- } \\
\text { dimensional gridded wind field in CALMET. This } \\
\text { parameter allows the user to select the method of } \\
\text { extrapolation: } 1 \text { (no extrapolation), } 2 \text { (power-law), } 3 \\
\text { (user-factors), } 4 \text { (similarity theory). Out of the four } \\
\text { methods, the power-law or similarity theory methods of } \\
\text { extrapolation are recommended. Only experienced } \\
\text { users should employ the user factors, as it requires a } \\
\text { thorough understanding of the site to use correctly. } \\
\text { The negative sign preceding the value of IEXTRP has } \\
\text { special meaning-it directs CALMET to ignore the } \\
\text { first-level winds in the upper-air data when creating the } \\
\text { surface layer wind field (i.e., use surface station data } \\
\text { only). }\end{array}$ \\
\hline
\end{tabular}




\begin{tabular}{|c|c|c|c|}
\hline $\begin{array}{l}\text { CALMET } \\
\text { Parameter }\end{array}$ & $\begin{array}{c}\text { Allowed } \\
\text { Values }\end{array}$ & $\begin{array}{l}\text { Recommended } \\
\text { Value }\end{array}$ & Description \\
\hline ICALM & $\begin{array}{c}0(\mathrm{No}) \\
\text { or } \\
1 \text { (Yes) }\end{array}$ & 0 (No) & $\begin{array}{l}\text { Extrapolate surface winds even if calm- Under calm } \\
\text { conditions, surface winds may not provide a realistic } \\
\text { representation of the winds aloft, and extrapolation of } \\
\text { the surface winds may not be desirable. It is } \\
\text { recommended that the surface winds not be } \\
\text { extrapolated under calm conditions. }\end{array}$ \\
\hline $\begin{array}{l}\text { RMAX1 } \\
\text { RMAX2 }\end{array}$ & $\begin{array}{c}\text { User- } \\
\text { specified } \\
(\mathrm{km})\end{array}$ & $\begin{array}{c}\text { RMAX1 } \\
\text { (50 to } 100 \mathrm{~km}) \\
\text { RMAX2 } \\
(100 \text { to } 500 \mathrm{~km})\end{array}$ & $\begin{array}{l}\text { Maximum radius of influence over land-This } \\
\text { parameter is the radius of influence at the surface } \\
(\mathrm{RMAX} 1) \text { and aloft (RMAX2) that observed winds } \\
\text { have on derived winds at each grid node. CALMET } \\
\text { uses a } 1 / \mathrm{r}^{2} \text { interpolation procedure when merging } \\
\text { observations with the derived flow. These radii control } \\
\text { the maximum extent to include observations at each } \\
\text { node. }\end{array}$ \\
\hline LVARY & $\begin{array}{c}\mathrm{T} \text { (True) } \\
\text { or } \\
\mathrm{F}(\text { False })\end{array}$ & $\mathrm{F}$ & $\begin{array}{l}\text { Use varying radius of influence-This parameter } \\
\text { increases the radius of influence parameters (RMAX1 } \\
\text { and RMAX2) until an observation station is located and } \\
\text { can be used in the } 1 / \mathrm{r}^{2} \text { interpolation procedure. This } \\
\text { parameter in not recommended in general, especially in } \\
\text { areas with complex terrain where the slope flow } \\
\text { formulation should be relied on to estimate the wind } \\
\text { filed. }\end{array}$ \\
\hline $\begin{array}{l}\mathrm{R} 1 \\
\mathrm{R} 2\end{array}$ & $\begin{array}{c}\text { User- } \\
\text { specified } \\
(\mathrm{km})\end{array}$ & $\begin{array}{c}\mathrm{R} 1 \\
(0.1 \text { to } 5 \mathrm{~km}) \\
\mathrm{R} 2 \\
(0.1 \text { to } 10 \mathrm{~km})\end{array}$ & $\begin{array}{l}\text { Radius for Equal Weighting of Observations with } \\
\text { Node-derived Values-This parameter controls the } \\
\text { radius in which observations are weighted equally with } \\
\text { node-derived values at the surface (R1) and aloft (R2). } \\
\text { For complex terrain with calm conditions, these radii } \\
\text { should be set very small }(0.1) \text {. For less complex } \\
\text { terrain, or normal-to-high wind conditions, setting these } \\
\text { to } 1 / 20 \text { of the domain width (i.e., } 5 \mathrm{~km} \text { for a } 100-\mathrm{km} \\
\text { domain) is recommended. }\end{array}$ \\
\hline IOBR & $\begin{array}{c}0(\mathrm{No}) \\
\text { or } \\
1 \text { (Yes) }\end{array}$ & $\begin{array}{c}0 \\
\text { or } \\
1\end{array}$ & $\begin{array}{l}\text { Use O'Brien procedure to adjust vertical velocity-This } \\
\text { adjustment is used during the final step in the wind field } \\
\text { formulation in CALMET and sets the vertical wind } \\
\text { velocities to zero at the top layer of the model domain. } \\
\text { This generally has the effect of dampening the vertical } \\
\text { velocities at all layers of the model. Using this } \\
\text { adjustment is recommended unless there is a known } \\
\text { reason that vertical velocities should not be zero in the } \\
\text { top layer of the domain (e.g., land-sea breeze present). }\end{array}$ \\
\hline
\end{tabular}




\begin{tabular}{|c|c|c|c|}
\hline $\begin{array}{l}\text { CALMET } \\
\text { Parameter }\end{array}$ & $\begin{array}{c}\text { Allowed } \\
\text { Values }\end{array}$ & $\begin{array}{l}\text { Recommended } \\
\text { Value }\end{array}$ & Description \\
\hline TERRAD & $\begin{array}{c}\text { User- } \\
\text { specified } \\
(\mathrm{km})\end{array}$ & 1 to $20 \mathrm{~km}$ & $\begin{array}{l}\text { Radius of influence of terrain features-This value } \\
\text { determines the relative impact terrain will have on the } \\
\text { wind field. Under calm conditions and complex terrain, } \\
\text { this parameter should be large }(1 / 5 \text { of the domain width). } \\
\text { If the terrain is not expected to have an effect, or } \\
\text { modeling the terrain effects is not desirable, this value } \\
\text { can be set lower. }\end{array}$ \\
\hline
\end{tabular}

\section{A.2.3 Calpuff.inp}

The CALPUFF input file (Calpuff.inp) is located in the "StaticData" directory for each site within DUSTRAN and is the template input file for CALPUFF. Its contents are merged with select-user input from the DUSTRAN interface for each simulation. Many of the parameters contained within Calpuff.inp control certain aspects of the plume concentration and deposition calculations, including the dispersion processes. These parameters are not available from the DUSTRAN interface and can only be edited via the static file.

The static Calpuff.inp file normally contains the most ideal parameter settings for a given site. However, there may be times when the user wants to examine the effects of modifying certain parameter settings. Extreme caution should be used when changing any parameter in the static Calpuff.inp file, as it may cause unrealistic results. Each parameter in the Calpuff.inp is documented in detail in the CALPUFF user's guide (Scire et al. 2000a). The following discussion highlights those parameters that are generally considered the most important relative to affecting CALPUFF results. Generally, these parameters are from the section "INPUT GROUP: 2 - Technical Options," and control the plume dispersion calculations.

\begin{tabular}{|c|c|c|c|}
\hline $\begin{array}{l}\text { CALPUFF } \\
\text { Parameter }\end{array}$ & $\begin{array}{c}\text { Allowed } \\
\text { Values }\end{array}$ & $\begin{array}{l}\text { Recommended } \\
\text { Value }\end{array}$ & Description \\
\hline MGAUSS & $\begin{array}{l}0 \\
1\end{array}$ & 1 & $\begin{array}{l}\text { Vertical distribution used in the near field-The } \\
\text { initial plume distribution in the vertical can be } \\
\text { uniform }(0) \text { or have a Gaussian distribution (1). A } \\
\text { uniform distribution most likely is not representative } \\
\text { of an actual release, so a Gaussian distribution is } \\
\text { recommended. }\end{array}$ \\
\hline MCTADJ & $\begin{array}{l}0 \\
1 \\
2 \\
3\end{array}$ & 0 & $\begin{array}{l}\text { No terrain adjustment (0) is recommended. The other } \\
\text { options are an Industrial Source Complex model type } \\
\text { adjustment (1), a simple CALPUFF-type adjustment } \\
\text { (2), or a partial plume path adjustment (3). The } \\
\text { model default is the partial plume path adjustment. }\end{array}$ \\
\hline MCTSG & $\begin{array}{l}0 \\
1\end{array}$ & 0 & $\begin{array}{l}\text { Subgrid-scale complex terrain flag-this parameter } \\
\text { should be turned off }(0) \text {. }\end{array}$ \\
\hline MSLUG & $\begin{array}{l}0 \\
1\end{array}$ & 1 & $\begin{array}{l}\text { Near-field puffs modeled as elongated-The slug } \\
\text { model, which is used to elongate puffs near the } \\
\text { source, is recommended (1) and appears to provide }\end{array}$ \\
\hline
\end{tabular}




\begin{tabular}{|c|c|c|c|}
\hline $\begin{array}{l}\text { CALPUFF } \\
\text { Parameter }\end{array}$ & $\begin{array}{l}\text { Allowed } \\
\text { Values }\end{array}$ & $\begin{array}{l}\text { Recommended } \\
\text { Value }\end{array}$ & Description \\
\hline & & & $\begin{array}{l}\text { more accurate results near the source than no } \\
\text { elongation }(0) \text {. }\end{array}$ \\
\hline $\begin{array}{l}\text { MTRANS, } \\
\text { MTIP, } \\
\text { MSHEAR, and } \\
\text { MSPLIT }\end{array}$ & $\begin{array}{l}0 \\
1\end{array}$ & 0 & $\begin{array}{l}\text { There are several puff manipulation parameters } \\
\text { including plume rise (MTRANS), stack-tip } \\
\text { downwash (MTIP), vertical wind shear above stack } \\
\text { height (MSHEAR), and puff splitting (MSPLIT) that } \\
\text { can be employed in CALPUFF. }\end{array}$ \\
\hline $\begin{array}{l}\text { MCHEM, } \\
\text { MAQCHEM }\end{array}$ & $\begin{array}{l}0 \\
1\end{array}$ & 0 & $\begin{array}{l}\text { In DUSTRAN, particle concentrations are assumed to } \\
\text { decrease rapidly from dispersion and deposition } \\
\text { processes. Chemical mechanism (MCHEM) and } \\
\text { aqueous phase transformation (MAQCHEM) are } \\
\text { considered to be negligible, and the flags should be } \\
\text { set to zero (not used). }\end{array}$ \\
\hline $\begin{array}{l}\text { MWET, } \\
\text { MDRY }\end{array}$ & $\begin{array}{l}0 \\
1\end{array}$ & 1 & $\begin{array}{l}\text { Deposition from wet and/or dry processes can be } \\
\text { treated. It is recommended that deposition be } \\
\text { activated. }\end{array}$ \\
\hline MDISP & $\begin{array}{l}1 \\
2 \\
3 \\
4 \\
5\end{array}$ & 2 & $\begin{array}{l}\text { Method used to compute dispersion coefficients- } \\
\text { CALPUFF allows the user a variety of ways to } \\
\text { calculate dispersion coefficients. The authors } \\
\text { recommend calculating the dispersion coefficients } \\
\text { from micrometeorological values (2). This method } \\
\text { uses similarity theory values, such as the Monin- } \\
\text { Obukhov length and friction velocity, to estimate the } \\
\text { dispersion parameters. Other options include } \\
\text { calculating the dispersion coefficients from measured } \\
\text { values of turbulence (1), and using different forms of } \\
\text { the Pasquill-Gifford dispersion curves ( } 3 \text { through 5). } \\
\text { If the meteorological data are not sufficient to allow } \\
\text { the use of similarity theory, then one of the Pasquill- } \\
\text { Gifford formulations is recommended. }\end{array}$ \\
\hline MPARTL & $\begin{array}{l}0 \\
1\end{array}$ & 0 & $\begin{array}{l}\text { Partial plume penetration of elevated inversion-This } \\
\text { parameter will allow the plume to partially penetrate } \\
\text { a temperature inversion if the calculated stack plume } \\
\text { rise is higher than the temperature inversion (1). This } \\
\text { parameter is not recommended for DUSTRAN (0) } \\
\text { because it results in additional plume dilution. }\end{array}$ \\
\hline MESHDN & 1 & 1 & $\begin{array}{l}\text { Sampling grid nesting factor that must be } 1 \text { for this } \\
\text { implementation of DUSTRAN }\end{array}$ \\
\hline
\end{tabular}

\section{A.2.4 Calgrid.inp}

The CALGRID input file (Calgrid.inp) is located in the "StaticData" directory for each site within DUSTRAN and is the template input file for CALGRID. Its contents are merged with select-user input from the DUSTRAN interface for each simulation. Many of the parameters contained within Calgrid.inp control certain aspects of the plume concentration and deposition calculations, including the dispersion 
processes. These parameters are not available from the DUSTRAN interface and can only be edited via the static file.

The static Calgrid.inp file normally contains the most ideal parameter settings for a given site. However, there may be times when the user wants to examine the effects of modifying certain parameter settings. Extreme caution should be used when changing any parameter in the static Calgrid.inp file, as it may cause unrealistic results. Each parameter in the Calgrid.inp is documented in detail in the CALGRID user's guide (Scire et al. 1989). One general run control parameter in CALGRID that is not set by DUSTRAN is the number of time steps per hour (NTSUBTS). The model recommended default for this parameter is 3 . In high wind conditions, CALGRID may produce the following error: "array bounds exceeded." This error can be eliminated by increasing the value of NTSUBTS. It has been found that values of 20 and 30 are successful in preventing this error under high-wind-speed conditions.

\section{A.3 Site Geodatabase}

Each site in DUSTRAN has an Access database file that is used to maintain, store, and render model data in ArcMap. This file is a "geodatabase" and has the name of the site with an .mdb extension. The file is stored in the root directory for each site.

A geodatabase contains a series of tables that describe the data. In the case of DUSTRAN, there are tables describing the model domain, sources, and simulation results. The tables provide all the necessary information for rendering the data in ArcMap and contain many useful attributes that the user can query within ArcMap for identification and display purposes.

The following sections provide documentation on the structure of the tables used within the DUSTRAN geodatabase. Specifically, the tables for domains, sources (point, line, and area), and simulated results are specified. Although these tables are automatically created and managed by the DUSTRAN interface, they are documented here for users who wish to manipulate and query the data within ArcMap.

\section{A.3.1 Domains}

The Domains table of the geodatabase is used to store the rectangular shapes representing the domains used in a simulation as well as attribute data associated with the domains. Domains that have been added to a site will remain in the Domains table until they are deleted by using the "Delete Domain" button in the DUSTRAN interface. Once a domain has been added in DUSTRAN, it is stored in the database and can be used in any simulation. The following table lists the field names and data types that are stored in the Domains table along with a description for each of the fields.

\begin{tabular}{|l|l|l|}
\hline Field Name & Data Type & Description \\
\hline DomName & String & Name of domain within scenario. \\
\hline Easting & Floating Point & Universal Transverse Mercator Easting value. \\
\hline Northing & Floating Point & Universal Transverse Mercator Northing value. \\
\hline DomSize & Integer & Size of the domain in km. \\
\hline Shape_Length & Floating Point & Length of the domain in meters. (Calculated by ArcMap) \\
\hline Shape_Area & Floating Point & Area of the domain in square meters. (Calculated by ArcMap) \\
\hline
\end{tabular}




\section{A.3.2 PointSources Table}

The PointSources table of the geodatabase is used to store the shapes representing the point sources used in a simulation as well as attribute data associated with the point sources. Point sources that have been selected or added to a simulation but not saved in a "Scenario" are stored temporarily in this table and are removed once a new simulation begins. Sources that have been saved in a "Scenario" will remain in the table and will be available for use in future simulations. The following table lists the field names and data types that are stored in the PointSources table along with a description for each of the fields.

\begin{tabular}{|c|c|c|}
\hline Field Name & Data Type & \begin{tabular}{|l|} 
Description \\
\end{tabular} \\
\hline ScenName & String & Name of the scenario that the source is currently saved under. \\
\hline OScenName & String & Original name of scenario. \\
\hline SrcID & String & Name of the source. \\
\hline RelRate & Double & Release rate of the source. \\
\hline RelRateUnit & String & Unit associated with the release rate. \\
\hline StkHeight & Integer & Height of the release. \\
\hline StkHeightUnit & String & Unit of measure used for StkHeight (stack height). \\
\hline StkDiam & Integer & Diameter of the stack. \\
\hline StkDiamUnit & String & Unit of measure used for StkDiam (stack diameter). \\
\hline BaseElev & Integer & Elevation at the base of the stack. \\
\hline BaseElevUnit & String & Unit of measure used for BaseElev (base elevation). \\
\hline ExitTemp & Integer & Temperature of the stack release. \\
\hline ExitTempUnit & String & Unit of measure used for ExitTemp (exit temperature). \\
\hline ExitVel & Integer & Velocity of stack release. \\
\hline ExitVelUnit & String & Unit of measure used for ExitVel (exit velocity). \\
\hline BCross & Integer & Cross sectional area of buildings. \\
\hline BCrossUnit & String & Unit of measure used for BCross parameter. \\
\hline VPlume & Integer & Initial vertical plume size. \\
\hline VPlumeUnit & String & Unit of measure used for VPlume. \\
\hline HPlume & Integer & Initial horizontal plume size. \\
\hline HPlumeUnit & String & Unit of measure used for HPlume. \\
\hline SMonth & Integer & Starting month of the release. \\
\hline SDay & Integer & Starting day of the release. \\
\hline SYear & Integer & Starting year of the release. \\
\hline SHour & Integer & Starting hour of the release. \\
\hline EMonth & Integer & Ending month of the release. \\
\hline EDay & Integer & Ending day of the release. \\
\hline EYear & Integer & Ending year of the release. \\
\hline EHour & Integer & Ending hour of the release. \\
\hline ScenComment & String & Comments about the release. \\
\hline SpecieNames & String & Names of the species emitted at the source. \\
\hline SpecieChecked & String & Flags used to designate which species are used at the source. \\
\hline EmissionVals & Floating Point & The emission values for each specie emitted at the source. \\
\hline GeoDiameters & Floating Point & Geometric mass mean diameters for the species used at the sol \\
\hline
\end{tabular}




\begin{tabular}{|l|l|l|}
\hline Field Name & Data Type & Description \\
\hline GeoDeviations & Floating Point & Geometric standard deviations for the species used at the source. \\
\hline MolWeights & Floating Point & Molecular weights for the species used at the source. \\
\hline DepVelocities & Floating Point & Deposition velocities for the species used at the source. \\
\hline
\end{tabular}

\section{A.3.3 LineSources}

The LineSources table of the geodatabase is used to store the shapes representing the line sources used in a simulation as well as attribute data associated with the line sources. Line sources are stored in each record of the table as line segments. Line sources that have been selected or added to a simulation but not saved in a "Scenario" are only stored in this table temporarily and will be removed once a new simulation is started. Line sources that have been saved in a "Scenario" will remain in the table and will be available for use in future simulation runs. The following table lists the field names and data types that are stored in the LineSources table along with a description for each of the fields.

\begin{tabular}{|l|l|l|}
\hline Field Name & Data Type & Description \\
\hline ScenName & String & Name that is used to save current scenario. \\
\hline OScenName & String & Original name scenario was saved under. \\
\hline SrcID & String & Name of the source. \\
\hline NumVeh & Integer & Number of vehicles in scenario. \\
\hline SiltCont & Integer & Silt content of the soil found along the line source. \\
\hline SoilType & Integer & Soil type found along the line source. \\
\hline SoilMoist & Integer & Moisture of the soil found along the line source. \\
\hline Paved & String & String describing whether a road is paved. T = True, F = False. \\
\hline VehCode & Integer & Integer code denoting the type of vehicle selected. \\
\hline VWeight & Integer & The weight of a given vehicle. \\
\hline VWeightUnit & String & Unit of measurement used for VWeight (vehicle weight). \\
\hline VSpeed & Integer & Speed of the vehicle. \\
\hline VSpeedUnit & String & Unit of measure used for VSpeed (vehicle speed). \\
\hline DistTrav & Floating & Distance traveled by vehicle. \\
\hline SMonth & Point & \\
\hline SDay & Integer & Starting month of the release. \\
\hline SYear & Integer & Starting day of the release. \\
\hline SHour & Integer & Starting year of the release. \\
\hline EMonth & Integer & Ending month of the release. \\
\hline EDay & Integer & Ending day of the release. \\
\hline EYear & Integer & Ending year of the release. \\
\hline EHour & Integer & Ending hour of the release. \\
\hline ScenComment & String & Comments on the scenario. \\
\hline Shape_Length & Floating & Length of the line source in meters. (calculated by ArcMap) \\
\hline VehName & String & Name of vehicle used in scenario. \\
\hline HTL & Integer & Release height of the emissions above the ground. \\
\hline HTLUnit & String & Units used for the HTL parameter. \\
\hline
\end{tabular}




\begin{tabular}{|c|c|c|}
\hline Field Name & Data Type & Description \\
\hline ELEVL & Integer & Base elevation. \\
\hline ELEVLUnit & String & Unit of measure used for ELEVL. \\
\hline SpecieNames & String & Names of the species associated with the source. \\
\hline SpecieChecked & String & Flags used to designate which species are used at the source. \\
\hline EmissionVals & $\begin{array}{l}\text { Floating } \\
\text { Point }\end{array}$ & The emission values for each specie emitted at the source. \\
\hline EmissionModel & String & $\begin{array}{l}\text { Flag denoting if the emission model is to be used for calculating the } \\
\text { emission rates at the source. }\end{array}$ \\
\hline GeoDiameters & $\begin{array}{l}\text { Floating } \\
\text { Point }\end{array}$ & Geometric mass mean diameters for the species used at the source. \\
\hline GeoDeviations & $\begin{array}{l}\text { Floating } \\
\text { Point }\end{array}$ & Geometric standard deviations for the species used at the source. \\
\hline MolWeights & $\begin{array}{l}\text { Floating } \\
\text { Point }\end{array}$ & Molecular weights for the species used at the source. \\
\hline DepVelocities & $\begin{array}{l}\text { Floating } \\
\text { Point }\end{array}$ & Deposition velocities for the species used at the source. \\
\hline
\end{tabular}

\section{A.3.4 AreaSources}

The AreaSources table of the geodatabase is used to store the shapes representing the area sources used in a simulation as well as attribute data associated with the area sources. Area sources are stored in each record of the table as polygon shapes. Sources that have been selected or added to a simulation but not saved in a "Scenario" are only stored in this table temporarily and will be removed once a new simulation is started. Sources that have been saved in a "Scenario" will remain in the table and will be available for use in future simulations. The following table lists the field names and data types that are stored in the AreaSources table along with a description for each of the fields.

\begin{tabular}{|l|l|l|}
\hline Field Name & Data Type & Description \\
\hline ScenName & String & Current name of the sources scenario. \\
\hline OScenName & String & Original name of the sources scenario. \\
\hline SrcID & String & Name of the source. \\
\hline NumVeh & Integer & Number of vehicles in scenario. \\
\hline SiltCont & Integer & Silt content of the soil found in the area source. \\
\hline SoilType & Integer & Soil type found within the area source. \\
\hline SoilMoist & Floating & Moisture of the soil found within the area source. \\
\hline Point & String & Flag denoting whether the source is a paved surface. \\
\hline VehCode & Integer & Integer code denoting the type of vehicle selected. \\
\hline VWeight & Integer & The vehicle's weight in kilograms. \\
\hline VWeightUnit & String & Unit of measure used to describe weight. \\
\hline VSpeed & Integer & The vehicle's speed in kilometers per hour. \\
\hline VSpeedUnit & String & Units of measure used to describe speed. \\
\hline DistTrav & Integer & Distance traveled in scenario. \\
\hline SMonth & Integer & Starting month of the release. \\
\hline SDay & Integer & Starting day of the release. \\
\hline
\end{tabular}




\begin{tabular}{|c|c|c|}
\hline Field Name & Data Type & Description \\
\hline SYear & Integer & Starting year of the release. \\
\hline SHour & Integer & Starting hour of the release. \\
\hline EMonth & Integer & Ending month of the release. \\
\hline EDay & Integer & Ending day of the release. \\
\hline EYear & Integer & Ending year of the release. \\
\hline EHour & Integer & Ending hour of the release. \\
\hline ScenComment & String & Comments on the scenario. \\
\hline Shape_Length & \begin{tabular}{|l|} 
Floating \\
Point
\end{tabular} & Length of the source in meters. (calculated by ArcMap) \\
\hline Shape_Area & $\begin{array}{l}\text { Floating } \\
\text { Point }\end{array}$ & Area of the source in meters. (calculated by ArcMap) \\
\hline VehName & String & Name of the selected vehicle. \\
\hline HT & Integer & Height of the source above ground level. \\
\hline HTUnit & String & Units used for the HT parameter. \\
\hline ELEV & Integer & Elevation of ground (mMSL). \\
\hline ELEVUnit & String & Units used for the ELEV parameter. \\
\hline TEMPK & Integer & Temperature at the release. \\
\hline TEMPKUnit & String & Units used for the TEMPK parameter. \\
\hline WEFF & Integer & Effective rise velocity. \\
\hline WEFFUnit & String & Units used with the WEFF parameter. \\
\hline REFF & Integer & Effective radius for rise calculation. \\
\hline REFFUnit & String & Units used with the REFF parameter. \\
\hline SIGZ & Integer & Initial vertical spread. \\
\hline SIGZUnit & String & Units used with the SIGZ parameter. \\
\hline SpecieNames & String & Names of the species associated with the source. \\
\hline SpecieChecked & String & Flags used to designate which species are used at the source. \\
\hline EmissionVals & String & The emission values for each specie emitted at the source. \\
\hline EmissionModel & String & $\begin{array}{l}\text { Flag denoting if the emission model is to be used for calculating the } \\
\text { emission rates at the source. }\end{array}$ \\
\hline GeoDiameters & String & Geometric mass mean diameters for the species used at the source. \\
\hline GeoDeviations & String & Geometric standard deviations for the species used at the source. \\
\hline MolWeights & String & Molecular weights for the species used at the source. \\
\hline DepVelocities & String & Deposition velocities for the species used at the source. \\
\hline
\end{tabular}

\section{A.3.5 Simulation Table}

The Simulation table of the geodatabase is used to store the results generated by DUSTRAN. Processed results are stored in the database table as polylines representing the contour levels present in the results. Along with the shapes of the different contour lines, the table is also used to store the attributes associated with the contour levels, such as the name of the scenario used to generate the results as well as the model type used in the simulation. The following table lists the fields that are present in the Simulation table along with a description of the data that are stored in each of the fields. 


\begin{tabular}{|l|l|l|}
\hline Field Name & Data Type & Description \\
\hline RunName & String & Name of the given run. \\
\hline DomName & String & Name of the domain used in the simulation. \\
\hline RunAlias & String & Name the simulation run is saved under. \\
\hline PTSrcNames & String & Names of the point sources used in the simulation. \\
\hline LNSrcNames & String & Names of the line sources used in the simulation. \\
\hline ARSrcNames & String & Names of the area sources used in the simulation. \\
\hline ScenName & String & Name of the scenario associated with the sources used in the simulation. \\
\hline Comment & String & User comments. \\
\hline Type & String & $\begin{array}{l}\text { Name of the model used to generate the result and the type of the result, } \\
\text { e.g., concentration or deposition. }\end{array}$ \\
\hline Time & Integer & String used to represent the date and time used in the simulation. \\
\hline Contour & Integer & Contour level of the result. \\
\hline ID & Integer & Contour ID number. \\
\hline Iteration & Integer & Iteration count of the contour. \\
\hline DomSize & Integer & Size of the domain used in the simulation. \\
\hline Shape_Length & $\begin{array}{l}\text { Floating } \\
\text { Point }\end{array}$ & Length of the contour shape in meters. (Calculated by ArcMap) \\
\hline EmissionType & String & Specie type associated with the result contour. \\
\hline
\end{tabular}

\section{A.4 DUSTRAN Initialization File (DUSTRAN.ini)}

An initialization file is used to store the configuration settings for the DUSTRAN modeling system. The file, called DUSTRAN.ini, is read upon launching the DUSTRAN application. The file is formatted text and primarily holds a list of variables that store available sites and directory path information. The following is an example of the DUSTRAN.ini file followed by a description of the parameters found in the file.

\section{A.4.1 Example DUSTRAN.ini File}

[DUSTRAN]

Site_List $=$ Yakima

GIS_exe=C: Program Files $\backslash$ ArcGIS $\backslash$ Bin $\backslash$ ArcMap.exe

Install_dir $=\mathrm{C}:$ IDUSTRAN

GIS_dir $=\mathrm{C}:$ :DUSTRAN $\backslash$ Yakima $\backslash$ GISData

First_Run $=$ False

InterfaceMode $=$ Multiple

SystemName $=$ DUSTRAN

AgDispName $=$ SPRAYTRAN

Read_Me $=$ False

Current_site $=$ Yakima

APR_dir=

[Yakima]

CharCategories $=$ Soil, Vegetation

Soil=srztext

SoilSel=yes 
Vegetation $=$ owe $14 \mathrm{~d}$

VegetationSel=yes

Srztext $=$ C: $\backslash$ DUSTRAN $\backslash$ Yakima $\backslash$ TerDatalsrztext.csv

Owe14d=C: $\backslash$ DUSTRAN $\backslash$ Yakima $\backslash$ TerDatalowe14d.csv

\section{A.4.2 DUSTRAN.ini File and Parameter Description}

\begin{tabular}{|c|c|}
\hline $\begin{array}{l}\text { Parameter } \\
\text { Name }\end{array}$ & Parameter Description \\
\hline Site_List & $\begin{array}{l}\text { Comma-separated string containing the names of the sites currently registered with the } \\
\text { modeling system. }\end{array}$ \\
\hline GIS_exe & Directory path of the ArcMap executable. \\
\hline Install_dir & Directory path in which the DUSTRAN modeling system has been installed. \\
\hline GIS_dir & Directory path of the folder in which the .mxd file for the current site is stored. \\
\hline First_Run & $\begin{array}{l}\text { Flag used to determine if the modeling system has been executed since being installed. } \\
\text { If this is the first startup of the modeling system, the read me file will be displayed } \\
\text { automatically. }\end{array}$ \\
\hline InterfaceMode & $\begin{array}{l}\text { Flag used to determine if the modeling system will be able to access multiple sites or } \\
\text { only a single site. }\end{array}$ \\
\hline SystemName & $\begin{array}{l}\text { Name of the modeling system. This is the name that will be displayed on the toolbar } \\
\text { button and the main interface form. }\end{array}$ \\
\hline AgDispName & $\begin{array}{l}\text { Flag used to determine if the modeling system is to be used to make DUSTRAN or } \\
\text { SPRAYTRAN modeling runs. }\end{array}$ \\
\hline Read_Me & $\begin{array}{l}\text { Flag used to determine if the read me file is displayed automatically upon the start of } \\
\text { the modeling system. }\end{array}$ \\
\hline Current_site & Name of the last site opened in the modeling system. \\
\hline APR_dir & Directory path of the folder used to store Avenue script files. (Currently not used) \\
\hline CharCategories & $\begin{array}{l}\text { Data characteristic categories that have been entered for the simulation sites. By } \\
\text { default, each site will have the Soil and Vegetation categories present. Other } \\
\text { categories can be entered for a site using the appropriate utility in the DUSTRAN } \\
\text { interface. }\end{array}$ \\
\hline Soil & $\begin{array}{l}\text { Comma-separated string containing the file names of the soil characteristic files } \\
\text { entered for the site. File names are listed in order of priority. }\end{array}$ \\
\hline SoilSel & $\begin{array}{l}\text { Comma-separated string containing the flags denoting whether a soil characteristic file } \\
\text { is to be used in the simulation for the site. }\end{array}$ \\
\hline Vegetation & $\begin{array}{l}\text { Comma-separated string containing the file names of the vegetation characteristic files } \\
\text { entered for the site. File names are listed in order of priority. }\end{array}$ \\
\hline VegetationSel & $\begin{array}{l}\text { Comma-separated string containing the flags denoting whether a vegetation } \\
\text { characteristic file is to be used in the simulation for the site. }\end{array}$ \\
\hline Srztext & Full file path to the location of the soil characteristic file for the site. \\
\hline Owe14d & Full file path to the location of the vegetation characteristic file for the site. \\
\hline
\end{tabular}




\section{A.5 Emission Model Input and Output Files}

DUSTRAN automatically creates the input file needed for the dust emissions model based upon user input for line and area sources (in the case of vehicular dust "Source Emission") or the model domain ("Wind-blown Dust" emissions). An example dust emissions input file is provided in this section for documentation purposes only; the user does not need to create or edit this file as it generated by user input from the DUSTRAN interface. The emission model's output file is also provided in this section. The output file contains the emission values calculated for each line or area source that was input to the model. Users may want to review the output file at the end of a simulation for additional insight into relative magnitudes of the emissions from the various sources.

Estimating dust emissions using AP-42 formulations requires surface silt loading $\left(\mathrm{g} \mathrm{m}^{-2}\right)$ for paved roads and surface material silt content (\%) and surface material moisture content (\%) for unpaved roads. These quantities are set to default values of $12 \mathrm{~g} \mathrm{~m}^{-2}, 12 \%$ and $12 \%$, respectively, in the cal.par file (see Section A.2.1).

\section{A.5.1 Input File (Ldustinp.txt File)}

The ldustinp.txt file is a space-delimited text file that contains the input parameters used by the LineDust emission model. The parameters found within the file deal with the characteristics of the source such as the start location, stop location, silt content, soil type, and soil moisture of the path traveled. The file also contains information about the vehicles that are traveling along the source such as the vehicle ID, weight, speed, and distance traveled. All of the parameters found within the file are generated based upon user input through either the graphical user interface or the map display of the ArcMap application.

A sample Ldustinp.txt file is provided in the following section. For clarity, each line is numbered within the \{\} brackets. The line numbers are used to reference the table in Section A.5.1.2, which provides a detailed description of each line in the sample file. The \{\} brackets and line numbers do not exist in an actual Ldustinp.txt file.

\section{A.5.1.1 Example Ldustinp.txt Input File}

\section{$\{1\} 2$}

\{2\} 186.609570011024360 .66137799871186 .609570011024373 .24913392222010

\{3\} 21212120

\{4\} 11176012.587755923509625

$\{5\} 624455075.526535541057615$

\{6\} 186.609570011024373 .24913392222192 .4834214913854384 .99683688307010

\{7\} 21212120

\{8\} 11176013.13433119994015

\{9\} 624455078.805987199640510 


\section{A.5.1.2 Example Ldustinp.txt File Description}

\begin{tabular}{|l|l|}
\hline $\begin{array}{c}\text { Line } \\
\text { Number }\end{array}$ & Description \\
\hline 1 & Number of line segments for which calculations are to be made \\
& $\begin{array}{l}\text { Starting UTM X and Y } \\
\text { Ending UTM X and Y } \\
\text { Segment start and end time } \\
\text { Emission factor flag }(0=\text { DRI emission factors, 1 = EPA AP-42 emission factors })\end{array}$ \\
\hline 3,7 & $\begin{array}{l}\text { Number of vehicle types in a given segment } \\
\text { Surface silt loading on paved roads }(\mathrm{g} / \mathrm{m} 2) \\
\text { Surface material silt content on unpaved roads }(\%) \\
\text { Surface material moisture content on unpaved roads }(\%) \\
\text { Paved road flag }(0=\text { unpaved road, } 1=\text { paved road) }\end{array}$ \\
\hline $4,5,8,9$ & $\begin{array}{l}\text { Vehicle ID } \\
\text { Vehicle weight }(\mathrm{kg}) \\
\text { Vehicle speed }(\mathrm{km} / \mathrm{hr}) \\
\text { Vehicle distance traveled }(\mathrm{km}) \\
\text { Number of vehicles present for this vehicle type. }\end{array}$ \\
\hline
\end{tabular}

\section{A.5.2 Output File (Ldustout.txt File)}

Ldustout.txt is a space-delimited text file generated as output by the LineDust emission model and stored within the DUSTRAN root directory. The file contains the emission values calculated for each line source that was passed to the model as input. The emissions contained within the file are categorized by the type of emission as well as the sum of the emissions for each line source and the individual emissions generated by each vehicle type traveling the line source. The types of emissions present in the file include PM2.5, PM10, PM15, and PM30. The file also contains the input values used to generate the emissions.

A sample Ldustout.txt file is provided in the following section. For clarity, each line is numbered within the \{\} brackets. The line numbers are used to reference the table in Section A.5.2.2, which provides a detailed description of each line in the sample file. The \{\} brackets and line numbers do not exist in an actual Ldustout.txt file.

\section{A.5.2.1 Example Ldustout.txt Output File}

\section{$\{1\} 2$}

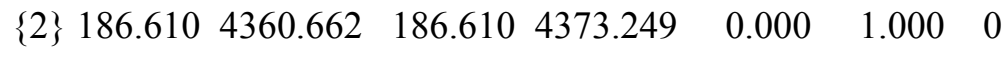

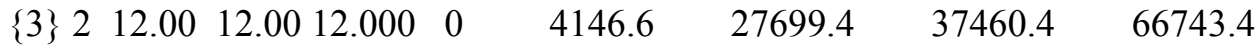

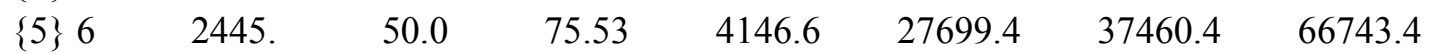

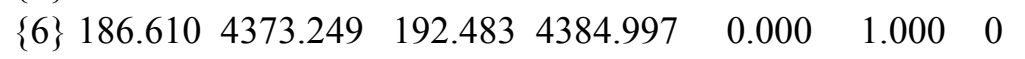

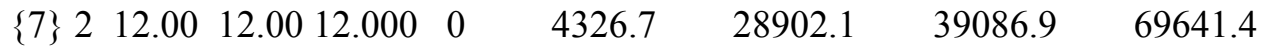

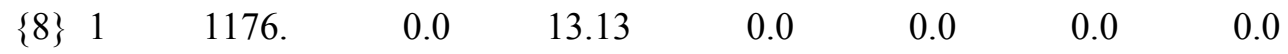

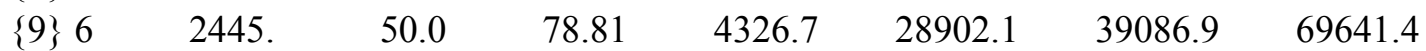




\section{A.5.1.2 Example Ldustout.txt File Description}

\begin{tabular}{|l|l|}
\hline $\begin{array}{c}\text { Line } \\
\text { Number }\end{array}$ & Description \\
\hline 1 & Number of line segments for which calculations are to be made \\
\hline 2 & $\begin{array}{l}\text { Starting UTM X and Y } \\
\text { Ending UTM X and Y } \\
\text { Segment start and end time } \\
\text { Emission factor flag }(0=\text { DRI emission factors, } 1=\text { EPA AP-42 emission factors })\end{array}$ \\
\hline 3,7 & $\begin{array}{l}\text { Number of vehicle types in a given segment } \\
\text { Surface silt loading on paved roads }\left(\mathrm{g} / \mathrm{m}^{2}\right)\end{array}$ \\
& $\begin{array}{l}\text { Surface material silt content on unpaved roads }(\%) \\
\text { Surface material moisture content on unpaved roads }(\%) \\
\text { Paved road flag }(0=\text { unpaved road, } 1=\text { paved road }) \\
\text { Total PM2.5, PM10, PM15, and PM30 emissions of indicated sizes from all vehicle }\end{array}$ \\
\hline $4,5,8,9$ & $\begin{array}{l}\text { Vehicle ID } \\
\text { Vehicle weight }(\mathrm{kg}) \\
\text { Vehicle speed }(\mathrm{km} / \mathrm{hr})\end{array}$ \\
& Vehicle distance traveled $(\mathrm{km})$ \\
& PM2.5, PM10, PM15, and PM30 emissions from individual vehicle type \\
\hline
\end{tabular}




\section{Distribution}

No. of

Copies

OFFSITE

1 SERDP Program Office Attention: John A. Hall

901 North Stuart Street, Suite 303

Arlington, VA 22203

(703) 696-2125

1 SERDP Support Office

HydroGeoLogic, Inc.

Attention: Susan Walsh

1155 Herndon Parkway, Suite 900

Herndon, VA 20170

(703) 326-7834

1 Sandy L. Bird

Environmental Protection Agency

National Exposure Research Laboratory

960 College Station Rd

Athens, GA 30605-2700

(706) $355-8318$

1 Harold W. Thistle

USDA Forest Service

180 Canfield Street

Morgantown, WV 26505

(304) 285-1574
No. of

Copies

ONSITE

28 Pacific Northwest National Laboratory

K.J. Allwine (15) K9-30

F.C. Rutz (5) K6-52

W.J. Shaw K9-30

J.P. Rishel K9-30

B.G. Fritz K6-75

E.G. Chapman K9-30

B.L. Hoopes K6-52

T.E. Seiple K6-52

Information Release (2) P8-55

Distr. 1 